\title{
Inverse-design and optimization methods for centrifugal pump impellers
}


Inverse-design and optimization methods for centrifugal pump impellers R.W. Westra

Printed by Gildeprint, Enschede

Thesis University of Twente, Enschede - With ref. - With summary in Dutch. ISBN 978-90-365-2702-6

copyright (c) 2008 by R.W.Westra, Enschede, The Netherlands 


\section{INVERSE-DESIGN AND OPTIMIZATION METHODS FOR CENTRIFUGAL PUMP IMPELLERS}

\section{PROEFSCHRIFT}

ter verkrijging van

de graad van doctor aan de Universiteit Twente, op gezag van de rector magnificus, prof. dr. W.H.M. Zijm,

volgens besluit van het College voor Promoties in het openbaar te verdedigen op vrijdag 5 september 2008 om 15.00 uur

door

Remko Willem Westra

geboren op 14 december 1976

te Zuidhorn 
Dit proefschrift is goedgekeurd door de promotor:

Prof.dr.ir. H.W.M. Hoeijmakers

en de assistent-promotor:

Dr.ir. N.P. Kruyt 


\section{Summary}

Due to the complex three-dimensional shape of turbomachines, their design is a delicate and difficult task. Small changes in geometrical details can lead to large changes in performance, like resulting head, efficiency and cavitation characteristics. In industry, turbomachines are often designed based on a combination of experience of the designer and direct Computational Fluid Dynamics (CFD) analyses of the flow inside these machines. The goal of this study is to develop advanced CFD-based design methods which can assist the designer in realizing better designs in shorter turn-around times.

The present work addresses the development of such CFD-based design methods for turbomachines. Both an inverse-design method and an optimization method have been developed. In particular, the developed methods can be applied to the design of machines for which the flow is assumed to be incompressible. As such, these methods are applicable to pumps, fans and hydraulic turbines. Furthermore, the core flow is considered to be inviscid and viscosity effects are assumed to be restricted to relatively thin boundary layers. In this thesis the focus is on the design of centrifugal pump impellers.

For the design methods developed in this thesis a potential flow method is employed, for which appropriate boundary conditions are formulated. This model is valid for flows that are inviscid, irrotational and incompressible. The Finite Element Method is utilized to solve the governing Laplace equation numerically. The augmented potential-flow model is discussed, which includes an estimate of the boundary layer losses in the impeller using a semi-empirical analysis of the inviscid flow field.

An inverse-design method for centrifugal pump impellers has been developed. For a direct method, the geometry of the impeller is used as input and the flow field and the performance are obtained as a result. In contrast, for an inverse method the performance is prescribed, via a loading function, and both the flow field and the blade curvature distribution are obtained as a result of the inverse-design analysis. Since the inverse-design method introduces an additional unknown, i.e. the blade curvature, an additional boundary condition is needed to solve the inverse-design problem. This is the so-called loading function on the blades. In this thesis it is given either by the mean-swirl distribution or by a velocity difference over the blades. By prescribing a suitable loading function, impellers are obtained with the prescribed pump head and zero incidence at the leading edge. The method has been verified and applied to the design of two three-dimensional impellers, a radial-flow type and a mixed-flow type impeller. For all inverse-designs improvements in the inception Net Positive Suction Head $\left(\mathrm{NPSH}_{i n c}\right)$ are obtained. This is the result of the prescribed zero-incidence at the leading edge. It is shown that by changing the build-up of the loading at the blades, performance parameters can be improved further. Generally, shifting the loading towards the trailing edge leads to an increase in blade length and boundary layer losses, as well as a decrease in $\mathrm{NPSH}_{i n c}$ and velocity loading. Also, shifting the loading towards the leading edge leads to a reduction in blade length and loss coefficient, but also to an increase in velocity loading and $\mathrm{NPSH}_{\text {inc }}$, combined with a higher risk of back-flow.

In addition to the inverse-design method, an optimization method for centrifugal pump impellers has also been developed. The direct optimization method employs a parame- 
terization of the impeller geometry, a formulation of the cost function that quantifies the performance of the design, and an optimization algorithm. In the parameterization part the geometry is parameterized in terms of a parameter vector $\boldsymbol{x}$ and appropriate bounds are selected. In the formulation of the cost function, relevant performance objectives are selected and weight factors for various flow rates and objectives are chosen such that the cost function $F(\boldsymbol{x})$ can be determined for each parameterized geometry. The cost function is evaluated for several flow rates around the design point, resulting in a multi-point optimization method. An optimization algorithm is utilized to solve the minimization problem and the optimum geometry is found with the lowest value for the cost-function. The method of Differential Evolution is employed to solve the minimization problem. This is an evolutionary method in which a population of geometries evolves over a number of generations towards the optimum. The developed method has been applied to the optimization of a radial pump impeller in which blade curvature, number of blades and shroud curvature are parameterized. The cost function incorporates objectives relating to cavitation characteristics, boundary layer losses, and pump head. A penalty factor is used for impellers with back-flow for the considered flow rate. For the selected range of number of blades an optimized impeller is obtained with improved cavitation characteristics. Additional optimizations show that a further improvement could have been obtained if a larger number of blades would have been allowed in the optimization. For the main optimization the number of blades has been bounded at a maximum of 6 impeller blades in order to have a good optical accessibility for Particle Image Velocimetry (PIV) measurements of the velocity field inside the impeller.

The optimization method has also been applied in combination with the inverse-design method. This combined approach is labeled inverse-optimization. Here, instead of a direct parameterization of the blade curvature distribution, the mean-swirl distribution is parameterized. The cost function includes the boundary layer losses, the velocity loading on the blades, the cavitation parameter NPSH and a penalty factor for back-flow. Only a single flow rate is considered for the inverse-optimization, making this approach a single-point optimization. The method of Differential Evolution is used once more as optimization algorithm. The inverse-optimization has been applied to the design of a mixed-flow impeller. The inverse-optimization results in an impeller with the blade loading at the shroud shifted towards the leading edge, and the blade loading at the hub shifted towards the trailing edge. The optimized impeller shows an improvement in NPSH and in the velocity loading on the blades.

The radial impeller that has been optimized with the direct optimization method, has been geometrically scaled, manufactured in perspex and installed in a newly designed, largely transparent experimental setup. The setup consists of a large cylindrical vessel filled with demineralized water. The impeller is attached to a hollow rotating cylinder, which is driven using a belt drive. The water flows through a central tube towards the impeller. At the upstream side of this central tube a spring valve is located that is used to control the flow rate separately from the rotational speed. A Venturi flow meter is used to measure this flow rate. For the measurements rotational speeds between 30 and $200 \mathrm{rpm}$ could be realized. Measurements above $200 \mathrm{rpm}$ were not possible due to the entrapment of air bubbles originating from the water-air interface. The operating range of the setup is between 0.3 and 1.9 times the design flow rate of the impeller, independent 
of the rotational speed. By adding small concentrations of polyamide seeding particles and using a digital camera, located in the rotating cylinder, PIV measurements have been carried out at 75 and 150rpm and for flow rates ranging from $50 \%$ to $150 \%$ of the design flow rate. A Nd:YAG laser is used to illuminate the seeding particles. The flow field has been measured in two planes perpendicular to the rotation-axis of the impeller, one near the hub and one near the shroud. The measurements in the plane near the hub show qualitative agreement with potential flow predictions, i.e. a low velocity at the pressure side and high velocity at the suction side of the blade. Quantitatively, the measured velocity in the plane near the hub is somewhat higher, however. In the plane near the shroud the velocity is lower than in the plane near the hub and they also differ from the potential flow predictions. In the plane near the shroud a jet-wake structure is clearly observed, featuring a large wake area of low relative velocity (but no back-flow) at the suction side of the blade, which is not predicted by the potential flow model. The suction side wake is observed in the plane near the shroud for all considered flow rates, and it is also seen in the plane near the hub for flow rates smaller than the design flow rate. Secondary flow theory can be used to explain the flow phenomena observed in the measurements. It is concluded that the potential flow model can be used adequately at the design point to predict global pump performance parameters like the pump head, but that for a more detailed and accurate description of the flow field a more sophisticated flow model is needed. 


\section{Samenvatting}

Het ontwerp van turbomachines is een ingewikkelde procedure, door de gecompliceerde drie-dimensionale vormen van turbomachines. Kleine veranderingen in geometrische details kunnen leiden tot grote veranderingen in prestaties, zoals de opvoerhoogte, rendement en cavitatie karakteristieken. In de industrie worden turbomachines dikwijls ontworpen gebaseerd op een combinatie van ervaring van de ontwerper en directe stromingsanalyses door middel van Computational Fluid Dynamics (CFD). Het doel van dit onderzoek is om geavanceerde ontwerpmethodes te ontwikkelen die gebaseerd zijn op CFD. Deze ontwerpmethodes kunnen de ontwerper helpen bij het realiseren van betere ontwerpen in kortere tijden.

Dit proefschrift behandelt de ontwikkeling van zulke op CFD-gebaseerde ontwerp methodes voor turbomachines. Zowel een inverse-ontwerp methode, als een optimalisatie methode zijn ontwikkeld. De ontwikkelde methodes kunnen worden toegepast voor het ontwerp van turbomachines, waarin de stroming incompressibel is. Daarom zijn de methodes geschikt voor het ontwerp van pompen, ventilatoren en hydraulische turbines. Er wordt aangenomen dat de hoofdstroming niet-viskeus is en dat viscositeitseffecten beperkt zijn tot relatief dunne grenslagen. Dit proefschrift concentreert zich op het ontwerp van centrifugaal waaiers voor pompen.

Voor de ontwerp methodes die ontwikkeld zijn in dit proefschrift wordt het potentiaal stromingsmodel gebruikt, waarvoor gepaste randvoorwaarden geformuleerd worden. Dit model is geldig voor stromingen die niet-viskeus, rotatievrij en incompressibel zijn. De Eindige Elementen Methode wordt aangewend om de geldende Laplace vergelijking numeriek op te lossen. Het uitgebreide potentiaal stromingsmodel wordt behandeld, waarin de grenslaag verliezen in de waaier worden berekend met een semi-empirisch beschouwing van het niet-viskeuze stromingsveld.

Een inverse-ontwerp methode voor centrifugaal waaiers is ontwikkeld. Voor een directe methode wordt de geometrie van de waaier als invoer gehanteerd en het stromingsveld en de prestaties worden als resultaat verkregen. Daarentegen, voor een inverse methode worden de prestaties opgelegd, via een bladbelasting, en zowel het stromingsveld als de bladkromming worden verkregen als resultaat van de inverse analyse. Er is een extra randvoorwaarde nodig voor het invers probleem, aangezien de inverse-ontwerp methode een extra onbekende introduceert, namelijk de bladkromming. Deze extra randvoorwaarde is de zogenaamde bladbelasting. In dit proefschrift wordt deze gegeven door middel van ofwel een 'mean-swirl' verdeling ofwel een snelheidsverschil op het bladoppervlak. Door een geschikte bladbelasting op te leggen kunnen waaiers ontworpen worden met de vereiste opvoerhoogte en schokvrije aanstroming aan de neus van het blad. De methode is geverifieerd en toegepast bij het ontwerp van twee driedimensionale waaiers, namelijk een radiale waaier en een 'mixed-flow' waaier. Voor alle inverse ontwerpen worden verbeteringen in 'Net Positive Suction Head' (NPSH) gevonden. Dit is het gevolg van de opgelegde schokvrije aanstroming aan de neus van het blad. Er wordt aangetoond dat door de opbouw van de blad belasting te veranderen, prestatie parameters verder verbeterd kunnen worden. In het algemeen geldt dat als de bladbelasting naar de staart van het blad 
verschoven wordt, de bladlengte en de grenslaag verliezen toenemen, terwijl de NPSH en de snelheidsbelasting aan het bladoppervlak afnemen. Als de bladbelasting verschoven wordt richting de neus van het blad, worden de bladlengte en grenslaag verliezen kleiner, terwijl de NPSH en de snelheidsbelasting aan het bladoppervlak toenemen, gecombineerd met een grotere kans op terugstroming.

Naast de inverse-ontwerp methode is tevens een optimalisatie methode voor centrifugaal waaiers ontwikkeld. De directe optimalisatie methode bestaat uit een parameterisatie van de waaier geometrie, een formulering van de kost functie, die de prestatie van een geometrie quantificeert en een optimalisatie algoritme. In het parameterisatie gedeelte wordt de waaier geometrie geparameteriseerd in termen van een parameter vector $\boldsymbol{x}$ en gepaste grenzen worden geselecteerd. In de formulering van de kost functie worden relevante prestatie doelen geselecteerd en weeg factoren worden gekozen zodat de kost functie $F(\boldsymbol{x})$ voor iedere geparameteriseerde geometrie bepaald kan worden. De kost functie wordt geëvalueerd op verschillende debieten rond het ontwerp debiet en dit resulteert in een multi-punt optimalisatie methode. Een optimalisatie algoritme wordt gebruikt om het minimalisatie probleem op te lossen en de optimale geometrie wordt verkregen met de kleinste waarde voor de kost functie. De methode van Differentiële Evolutie wordt gehanteerd om het minimalisatie probleem op te lossen. Dit is een evolutionaire methode waarin een populatie van geometriëen over een aantal generaties evolueert richting een optimum. De ontwikkelde methode is toegepast bij de optimalisatie van een radiale waaier van een pomp, waarbij de bladkromming, het aantal bladen en de kromming van het bovendeksel geparameteriseerd zijn. De kost functie omvat doelen gerelateerd aan cavitatie karakteristieken, grenslaag verliezen en opvoerhoogte. Een penalty factor wordt toegepast voor waaiers met terugstroming voor het beschouwde debiet. Voor het geselecteerde bereik van het aantal bladen is een geoptimaliseerde waaier verkregen met een verbetering in cavitatie karakteristieken. Extra optimalisaties hebben laten zien dat een verdere verbetering verkregen kan worden indien een groter aantal bladen zou zijn toegelaten in de optimalisatie. Voor de hoofd optimalisatie is het aantal bladen begrensd op maximaal 6 opdat een goede optische toegankelijkheid wordt bewerkstelligd voor de 'Particle Image Velocimetry' (PIV) metingen van het snelheidsveld in de waaier.

De optimalisatie methode is tevens in combinatie met de inverse-ontwerp methode toegepast. Deze gecombineerde aanpak wordt inverse-optimalisatie genoemd. Hier wordt de 'mean-swirl' verdeling geparameteriseerd, in plaats van een directe parameterisatie van de bladkromming. De kost functie omvat grenslaag verliezen, de snelheidsbelasting van de bladen, de cavitatie parameter NPSH en een penalty factor voor terugstroming. Slechts één debiet wordt beschouwd in de inverse-optimalisatie, waardoor deze aanpak resulteert in een enkel-punt optimalisatie. Wederom wordt de methode van Differentiële Evolutie gehanteerd als optimalisatie algoritme. De inverse-optimalisatie is toegepast bij het ontwerp van een 'mixed-flow' waaier. De inverse-optimalisatie resulteert in een waaier, waarvoor de bladbelasting aan het bovendeksel is verschoven naar de neus van het blad en de bladbelasting aan het onderdeksel is verschoven naar de staart van het blad. De geoptimaliseerde waaier vertoont een verbetering in NPSH en snelheidsbelasting op de bladen.

De radiale waaier die geoptimaliseerd is met de directe optimalisatie methode is geometrisch geschaald, vervaardigd in perspex en toegevoegd aan een nieuw ontworpen, gro- 
tendeels transparante, experimentele opstelling. De opstelling bestaat uit een groot cilindrisch vat gevuld met gedemineraliseerd water. De waaier is bevestigd aan een holle roterende cilinder, welke aangedreven wordt door middel van een $\mathrm{V}$-snaar. Het water stroomt door een centrale buis naar de waaier. Stroomopwaarts van deze centrale buis bevindt zich een regelklep in de vorm van een veer, waarmee het debiet kan worden ingesteld, onafhankelijk van de rotatie snelheid. Een Venturi debiet meter wordt gehanteerd om dit debiet te kunnen meten. Voor de metingen konden rotatie snelheden tussen de 30 en 200rpm gerealiseerd worden. Metingen boven 200rpm waren niet mogelijk doordat luchtbellen afkomstig van het water-lucht interface in de opstelling kwamen. Het werkgebied van de opstelling ligt tussen de 0.3 en 1.9 keer het ontwerp debiet van de waaier, onafhankelijk van de rotatie snelheid. Door kleine concentraties polyamide deeltjes toe te voegen en gebruik te maken van een digitale camera, gelokaliseerd in de roterende cilinder, konden PIV metingen uitgevoerd worden op 75 en 150rpm voor debieten variërend van $50 \%$ tot $150 \%$ van het ontwerp debiet. Een Nd:YAG laser is gebruikt om de PIV deeltjes te belichten. Het stromingsveld is gemeten in twee vlakken loodrecht op de rotatie as van de waaier, een bij het onderdeksel en een bij het bovendeksel van de waaier. De metingen in het vlak bij het onderdeksel laten een kwalitatieve overeenkomst zien met de berekeningen op basis van het potentiaal stromingsmodel, d.w.z. een lage snelheid aan de drukzijde en een hoge snelheid aan de zuigzijde van het blad. Kwantitatief gezien echter zijn de snelheden in het vlak bij het onderdeksel enigszins hoger dan voorspeld door de berekeningen. In het vlak bij het bovendeksel zijn de gemeten snelheden lager dan bij het onderdeksel en tevens is er een verschil met de berekende snelheden. In het vlak bij het bovendeksel wordt een zogenaamde 'jet-wake' structuur waargenomen, wat onder andere bestaat uit een zog gebied van lage snelheid (maar geen terugstroming) aan de zuigzijde van het blad, wat niet voorspeld wordt door het potentiaal stromingsmodel. Het lage snelheidsgebied aan de zuigzijde wordt waargenomen in het vlak bij het bovendeksel voor alle beschouwde debieten en het is tevens te zien in het vlak bij het onderdeksel voor debieten kleiner dan het ontwerp debiet. Secondaire-stromings theorie kan gebruikt worden om de waargenomen stromingspatronen te verklaren. Er wordt geconcludeerd dat het potentiaal stromingsmodel gehanteerd kan worden in het ontwerp punt om globale pomp prestatie parameters zoals de opvoerhoogte te voorspellen, maar dat voor een gedetailleerdere beschrijving van het stromingsveld een meer geavanceerd stromingsmodel nodig is. 
Summary

Samenvatting $\quad$ iv

Table of Contents vii

1 Introduction 1

1.1 Centrifugal pumps . . . . . . . . . . . . . . . . . . 1

1.1.1 Centrifugal pump components . . . . . . . . . . . . . 2

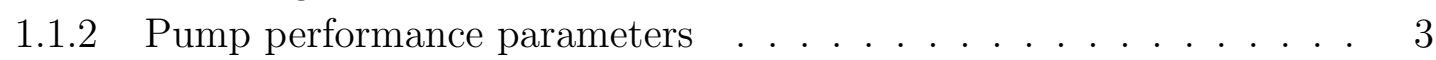

1.1.3 Meridional geometry . . . . . . . . . . . . . . . . . . . . . 4

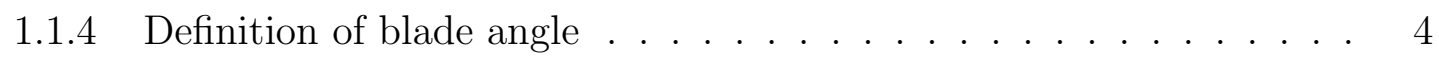

1.1.5 Dimensionless coefficients . . . . . . . . . . . . . 5

1.2 Basic pump analysis . . . . . . . . . . . . . . . . . . . 8

1.3 Objective and outline . . . . . . . . . . . . . . . . . . 11

2 Potential Flow Model $\quad 13$

2.1 Flow model . . . . . . . . . . . . . . . . . . 13

2.1.1 Absolute frame of reference . . . . . . . . . . . . . . . . 13

2.1.2 Rotating frame of reference . . . . . . . . . . . . . . 15

2.2 Boundary conditions . . . . . . . . . . . . . . . . . 16

2.3 Augmented potential flow model . . . . . . . . . . . . . . . . 19

2.4 Numerical method . . . . . . . . . . . . . . . . . . . 20

2.4.1 Weak form of the Laplace equation . . . . . . . . . . . . . . . 20

2.4.2 Structured mesh generation . . . . . . . . . . . . . . . . . . 21

2.4.3 Finite Element Method . . . . . . . . . . . . . . . . . . . . 21

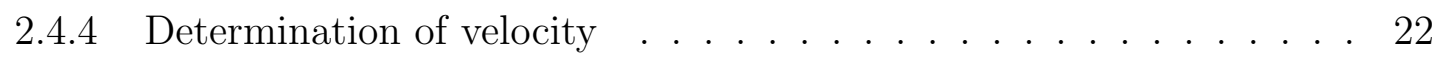


3 Inverse-design Method $\quad 25$

3.1 Literature overview . . . . . . . . . . . . . . . . . . . . . 25

3.2 Inverse-design method . . . . . . . . . . . . . . . 27

3.2.1 Design conditions . . . . . . . . . . . . . . . . 27

3.2.2 Curvilinear coordinate system . . . . . . . . . . . . . . . . . 28

3.2 .3 Mean-swirl distribution . . . . . . . . . . . . . . . . . 28

3.2 .4 Inverse-design algorithm . . . . . . . . . . . . . . . . . . . 31

3.2.5 Impenetrability condition . . . . . . . . . . . . . . . . 31

3.3 Numerical Implementation . . . . . . . . . . . . . . . . . . . . . . . . . . . . . . . . . . . . 34

3.3.1 Quasi 3D initial estimate . . . . . . . . . . . 35

3.3.2 Flow solution . . . . . . . . . . . . . . . . . . 35

3.3.3 Blade shape adjustment . . . . . . . . . . . . . . . . 36

3.3.4 Comparison with other methods . . . . . . . . . . . . . 37

3.4 Verification cases . . . . . . . . . . . . . . . . . 38

$3.4 .12 \mathrm{D}$ source with vortex . . . . . . . . . . . . 38

3.4.2 Reproducing a logarithmic blade . . . . . . . . . . . . . . . . 39

3.4.3 Order of accuracy . . . . . . . . . . . . . . . . . . . 42

3.5 Inverse-design of radial impeller blades . . . . . . . . . . . . . . . . . . . . . . . . . . . . . . . 43

3.5.1 SHF impeller original design . . . . . . . . . . . . . . . . . . . . . . 43

3.5.2 SHF impeller inverse-design case $1 \ldots \ldots$. . . . . . . . . . . 46

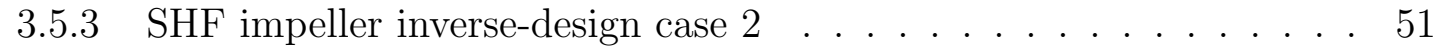

3.5.4 Comparison of SHF impeller designs . . . . . . . . . . . . . . . 52

3.6 Inverse-design of mixed-flow impeller blades . . . . . . . . . . . . . . . 55

3.6.1 Mixed-flow impeller original design . . . . . . . . . . . . . . 55

3.6.2 Mixed-flow impeller inverse-design case $1 \ldots$. . . . . . . . . . . 57

3.6.3 Mixed-flow impeller inverse-design case $2 \ldots \ldots$. . . . . . . . . . . . 59

3.6.4 Comparison of mixed-flow impeller designs . . . . . . . . . . . . . . 62

3.7 Alternative specification of loading . . . . . . . . . . . . . . . 64

3.7.1 Derivative of the mean-swirl distribution . . . . . . . . . . . . 64

3.7.2 Velocity difference distribution . . . . . . . . . . . . . . . . . . 64

3.7.3 Application of a velocity difference distribution . . . . . . . . . . 65

3.8 Discussion and conclusions . . . . . . . . . . . . . . . . . . . 68

4 Optimization Method $\quad 71$

4.1 Introduction to optimization . . . . . . . . . . . . . . . . 72

4.1 .1 Literature overview . . . . . . . . . . . . . . . . . . 72

4.1.2 Requirements for the optimization algorithm . . . . . . . . . . 73

4.2 Differential Evolution . . . . . . . . . . . . . . . . . 74

4.2.1 Verification for test problems . . . . . . . . . . . . . . . . . . 75

4.2.2 Applied numerical algorithm . . . . . . . . . . . . . . . . 76

4.3 Optimization of a radial centrifugal pump impeller . . . . . . . . . . . . 77

4.3 .1 Original impeller . . . . . . . . . . . . . . . . . . 77

4.3 .2 Parameterization . . . . . . . . . . . . . . . 78

4.3 .3 Cost function . . . . . . . . . . . . . . . . . . . . 79

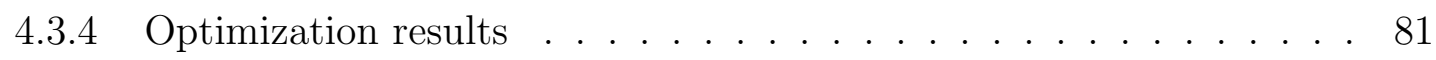


4.3.5 Additional optimizations . . . . . . . . . . . . . . . . 86

4.4 Inverse-optimization of a mixed flow impeller . . . . . . . . . . . . . . . 91

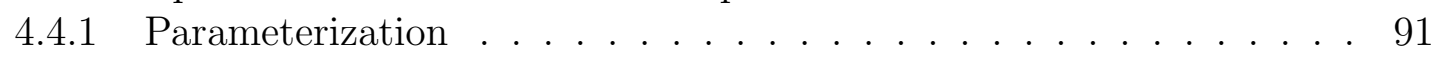

4.4 .2 Cost function . . . . . . . . . . . . . . . . . . . . 92

4.4 .3 Optimization result . . . . . . . . . . . . . . . . . . 92

4.5 Discussion . . . . . . . . . . . . . . . . . . . 96

$5 \quad$ PIV-measurements in an optimized impeller $\quad 99$

5.1 Experimental setup . . . . . . . . . . . . . . . . . . . . 100

5.2 Operational aspects . . . . . . . . . . . . . . . . . . 101

5.2 .1 Venturi flow meter . . . . . . . . . . . . . . . . . 101

5.2 .2 Operating range of the experimental setup . . . . . . . . . . . . . 104

5.2 .3 Pressure drop over the impeller . . . . . . . . . . . . . . . . . . . . 104

5.3 Particle Image Velocimetry ． . . . . . . . . . . . . . . . . . . 107

5.3.1 Literature overview . . . . . . . . . . . . . . . . . 107

5.3 .2 PIV principle . . . . . . . . . . . . . . . . . 108

5.3.3 PIV parameters . . . . . . . . . . . . . . . . . 109

5.4 PIV measurement quality . . . . . . . . . . . . . . . . . 115

5.4 .1 PIV images . . . . . . . . . . . . . . . . . . . 115

5.4 .2 Peak locking . . . . . . . . . . . . . . . . . . 116

5.4 .3 Time averaging . . . . . . . . . . . . . . . . . . 119

5.4 .4 Reproducibility . . . . . . . . . . . . . . . 120

5.5 Measurement results for design conditions . . . . . . . . . . . . 122

5.5.1 Influence of Reynolds number . . . . . . . . . . . . . . . . . . 126

5.6 Measurement results at off-design conditions . . . . . . . . . . . . . . 128

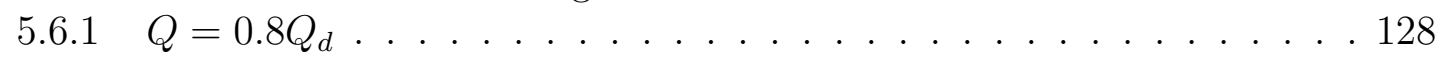

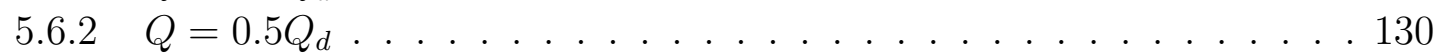

$5.6 .3 Q=1.2 Q_{d} \ldots \ldots \ldots \ldots 132$

$5.6 .4 Q=1.5 Q_{d} \ldots \ldots \ldots \ldots \ldots$

5.7 Discussion . . . . . . . . . . . . . . . . . . . 136

5.7 .1 Secondary flow . . . . . . . . . . . . . . . . 136

5.7 .2 Comparison with literature . . . . . . . . . . . . . . . 141

5.8 Conclusions and recommendations . . . . . . . . . . . . . . . . . . . . 143

$\begin{array}{lll}6 & \text { Discussion } & 145\end{array}$

$\begin{array}{lr}\text { Bibliography } & 149\end{array}$

$\begin{array}{ll}\text { A Sensitivity analysis of optimization parameters } & 157\end{array}$

B Vector plots of relative velocity $\quad 159$

$\begin{array}{ll}\text { C Velocity measurements in centrifugal impellers } & 165\end{array}$ 

CHAPTER 1

Introduction

The development of methods that support the design of turbomachines, and more specifically centrifugal pumps and fans, is the main objective of this thesis. In this first chapter an introduction to centrifugal pumps is given. Firstly, some important centrifugal pump characteristics are discussed. Secondly, a 1D performance analysis is given for radial pumps, in order to show the main parameters that determine turbomachine performance. The objective and outline of the thesis are given in the final section.

\subsection{Centrifugal pumps}

A pump or a fan is a machine that, by increasing the pressure of a fluid, is used to transport liquids or gasses, respectively. The focus in this thesis will be on pumps, but the principles for fans are very similar. Usually two types of pumps can be distinguished, centrifugal pumps and positive displacement pumps. In centrifugal pumps energy is transferred directly to the fluid by the contact between the rotating blades and the fluid. In positive displacement pumps a portion of fluid is trapped and moved in a given direction. The famous Archimedes screw, invented in the 3rd century B.C., is an example of a positive displacement pump. In this thesis centrifugal turbomachines are considered.

In this section some general pump definitions and terminology are presented. Firstly, the centrifugal pump components are discussed. Secondly, important pump performance characteristics are given. Furthermore, the meridional geometry, which plays an important role in pump design, is described. The blade angle definition is given thereafter. Dimensionless coefficients, which are frequently used for scaling purposes, are given at the end of this section. 


\subsubsection{Centrifugal pump components}

In Fig. 1.1 a typical centrifugal pump is displayed. A centrifugal pump consists of an impeller, a diffuser and a casing. An impeller consists of a rotating disc called the hub, to which blades are attached. The impeller is attached to an axis, which is driven by a motor. The rotating motion of the impeller blades moves the fluid outwards. Examples of impellers are shown in Fig. 1.1b and Fig. 1.2.

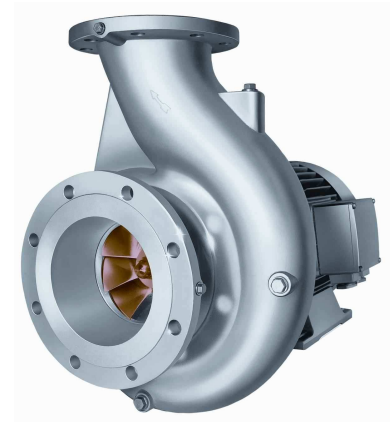

(a) view from the outside

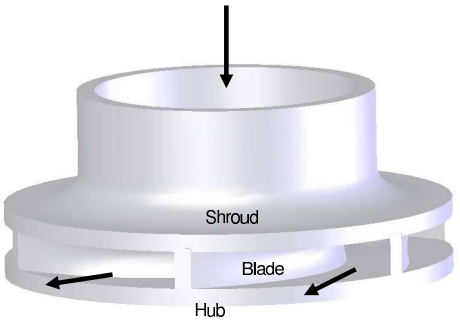

(b) rotating parts

Figure 1.1: Centrifugal pump. The arrows in (b) indicate the flow direction. Left picture taken from [72].

Impellers are frequently classified as either unshrouded or shrouded, both types are illustrated in Fig. 1.2. In shrouded impellers the blade tips are attached to the shroud surface, consequently the shroud rotates with the hub and the blades. In unshrouded impellers the tip of the blades has a small clearance with the stationary shroud. In this thesis shrouded impellers are considered, unless mentioned otherwise.

When the fluid leaves the impeller it enters the diffuser, where a large part of the dynamic pressure is converted into static pressure. Diffusers are either vaned diffusers, containing stationary blades (or vanes), or vaneless diffusers, which do not have these blades. In this thesis the focus will be on the design of impellers, but it has to be stressed

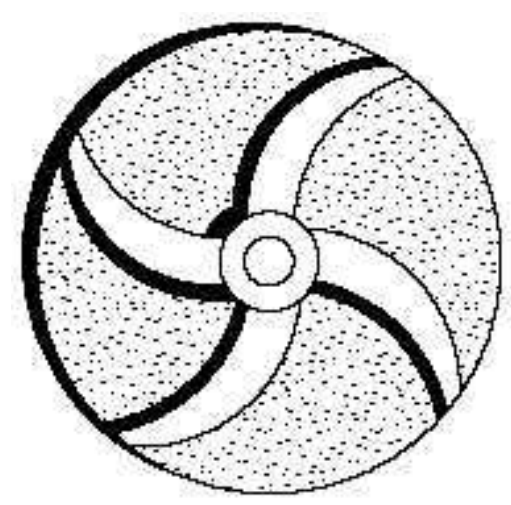

(a) unshrouded

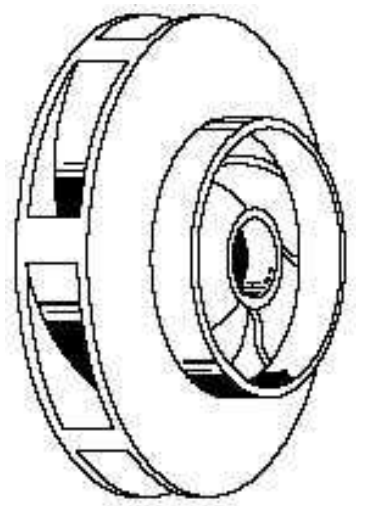

(b) shrouded

Figure 1.2: Unshrouded and shrouded impeller types. Picture taken from [29]. 
that diffuser design is very important as well.

\subsubsection{Pump performance parameters}

Pumps are usually designed to operate at a certain design flow rate $Q$ and an angular speed $\Omega$. At these conditions the pump generates an increase in stagnation pressure $\Delta p_{0}$, which is expressed in terms of a pump head $H$

$$
H=\frac{\Delta p_{0}}{\rho g}
$$

where $\rho$ is the density of the fluid, $g$ the gravitational acceleration and the stagnation pressure $p_{0}$ is given by

$$
p_{0}=p+\frac{1}{2} \rho v^{2}
$$

where $p$ is the static pressure and $v$ the fluid velocity.

Another important performance parameter is the power supplied to the pump via the shaft, $P_{S}$. The net hydraulic power transferred by the pump to the fluid $P_{H}$ is obtained from the pump head and the flow rate

$$
P_{H}=\rho g H Q
$$

Since losses occur, the hydraulic power $P_{H}$ is always smaller than the shaft power $P_{S}$. These losses can be divided in mechanical losses and hydraulic losses. Mechanical losses are friction related losses, like for example in bearings and seals. Hydraulic losses include leakage losses, dissipation in boundary layers, mixing losses and disc friction. This leads to another important pump performance parameter, the pump efficiency $\eta$. The pump efficiency is readily obtained from the shaft power and the hydraulic power

$$
\eta=\frac{P_{H}}{P_{S}}
$$

A further important phenomenon that may occur in pumps is that of cavitation. If the pressure of the liquid $p$ drops below the vapor pressure $p_{v}$ of the liquid, bubbles start forming and even sheets of vapor arise on the blades. Since the pressure in the pump increases whilst moving from the inlet towards the outlet, these gas pockets will collapse again to form liquid. This can cause severe damage to the impeller, called cavitation erosion. An example of a pump impeller affected by cavitation erosion is given in Fig. 1.3. Not only does cavitation lead to a reduction in pump life time, but the occurrence of cavitation also leads to a drop in pump head and efficiency, noise generation and vibrations. Therefore it is important to avoid cavitation as much as possible.

Parameters influencing the occurrence of cavitation in pumps for given flow rate and rotational speed are the vapor pressure of the liquid, $p_{v}$, the stagnation pressure at the inlet of the pump $p_{0, i n}$ and the geometry of the pump impeller. Note that the vapor pressure is a function of the temperature. The NPSH, Net Positive Suction Head, is used to indicate the over-pressure needed at the inlet of the pump to avoid cavitation. $\mathrm{NPSH}_{i n c}$, the cavitation inception criterion, is defined by 


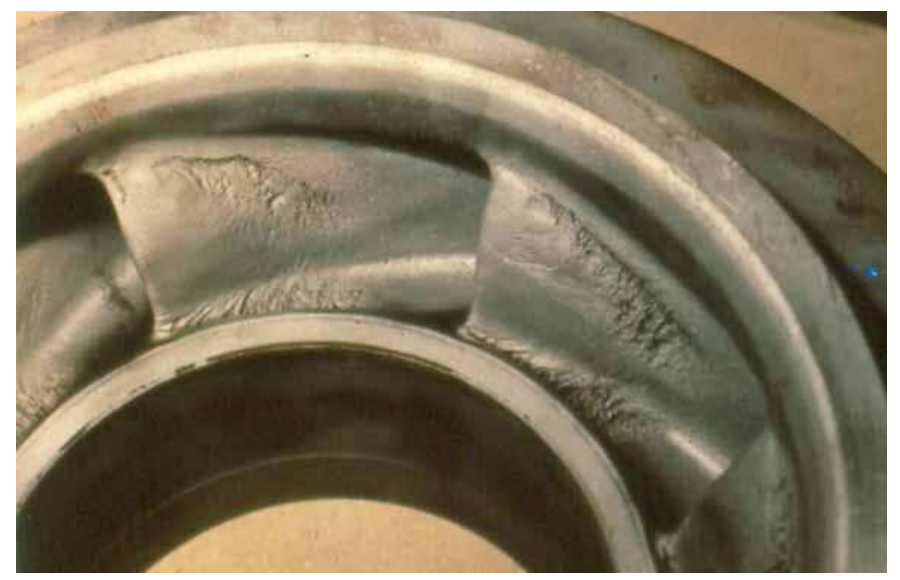

Figure 1.3: Cavitation erosion in a centrifugal pump. Picture taken from [7].

$$
\mathrm{NPSH}_{i n c}=\frac{p_{0, i n}^{*}-p_{v}}{\rho g}
$$

where $p_{0, \text { in }}^{*}$ is the value of the stagnation pressure at the inlet of the pump at which the first cavitation bubbles inside the pump start to form. A low $\mathrm{NPSH}_{\text {inc }}$ value is therefore desirable for design purposes, since the lower the $\mathrm{NPSH}_{\text {inc }}$ the lower the pressure can be at the inlet of the pump, while still avoiding cavitation.

Several NPSH criteria are used and the ones most frequently used are given here. Firstly, the $\mathrm{NPSH}_{i n c}$, which gives the NPSH value at cavitation inception, as discussed above. Another frequently used criterion in industry is that of $\mathrm{NPSH}_{3 \%}$, which is the NPSH value for which the pump head in cavitating condition is 3 percent less than that without the occurrence of cavitation. Usually at such a drop in pump head the pump is already severely cavitating, since $\mathrm{NPSH}_{i n c}>\mathrm{NPSH}_{3 \%}$.

\subsubsection{Meridional geometry}

A centrifugal pump rotates around an axis, here taken as the $z$-axis, and therefore it is often convenient to employ a cylindrical coordinate system $r, \theta, z$. A very useful and frequently used projection of an impeller blade is the so-called meridional geometry of an impeller blade. This is an $r, z$-projection of the blade. An example of a meridional geometry is given in Fig. 1.4, where $m$ is the non-dimensional meridional distance along a blade contour in the meridional plane from leading to trailing edge. Hence, at the leading edge $m=0$ and at the trailing edge $m=1$. Note that not only the blade is shown in this meridional view, but also an inlet and an outlet section.

\subsubsection{Definition of blade angle}

Pump impeller blades usually have complicated curved shapes and a common way to describe this shape is to define an impeller blade angle. In this thesis the blade angle $\beta$ is defined as a function of the meridional direction $m$, as sketched in Fig. 1.5. The 


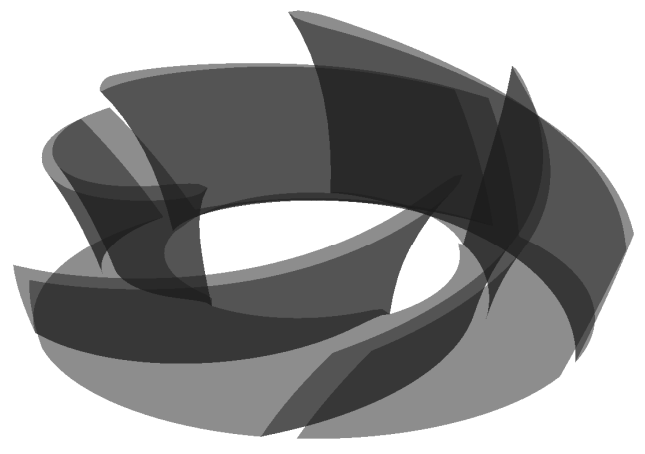

(a) full impeller view

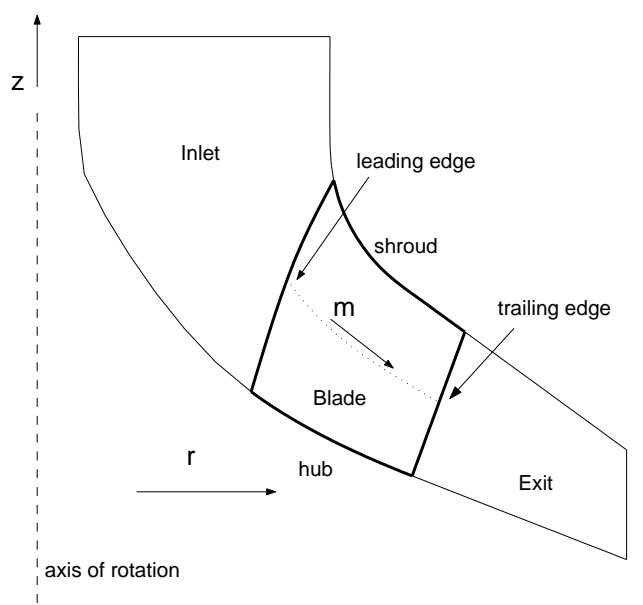

(b) meridional view

Figure 1.4: Example of an impeller and its corresponding meridional geometry. Note that for the full impeller view only the blade section is shown.

blade angle $\beta$ is the angle between the blade contour and the circumferential direction, i.e. a circular arc around the axis of rotation. The blade contour is an intersection of the blade surface with the surface of revolution of a meridional line (see Fig. 1.5). For a three-dimensional geometry the blade angle is given by

$$
\tan \beta=\frac{1}{r} \frac{d x_{m}}{d \theta}
$$

where $d x_{m}$ is the infinitesimal arc length in the meridional direction $m\left(d x_{m}=\sqrt{d r^{2}+d z^{2}}\right)$, see also Fig. 1.4. For a two-dimensional configuration $d z=0$ and therefore, $d x_{m}=d r$. Note that $m$ is dimensionless, whereas $x_{m}$ has the dimension of length. The variation of the blade angle $\beta$ reflects the blade curvature and hence the blade shape. The blade angle also occurs in the 1D flow analysis presented in section 1.2.

\subsubsection{Dimensionless coefficients}

Scaling of machines, whilst maintaining favorable performance characteristics, e.g. a maximum efficiency $\eta$, is important in the field of turbomachines. Dimensionless performance coefficients are often used to describe the performance parameters introduced in section 1.1.2. One such coefficient is the flow coefficient $\phi$, which gives the dimensionless flow rate

$$
\phi=\frac{Q}{\Omega D^{3}}
$$

where $D$ is the impeller outer diameter. The pump head is usually given in the form of a head coefficient $\psi$

$$
\psi=\frac{g H}{\Omega^{2} D^{2}}
$$

For a fixed rotational speed $\Omega$ turbomachines operate with a maximum efficiency $\eta$ for a certain flow rate $Q$ and a corresponding pump head $H$. Based on the flow and head 


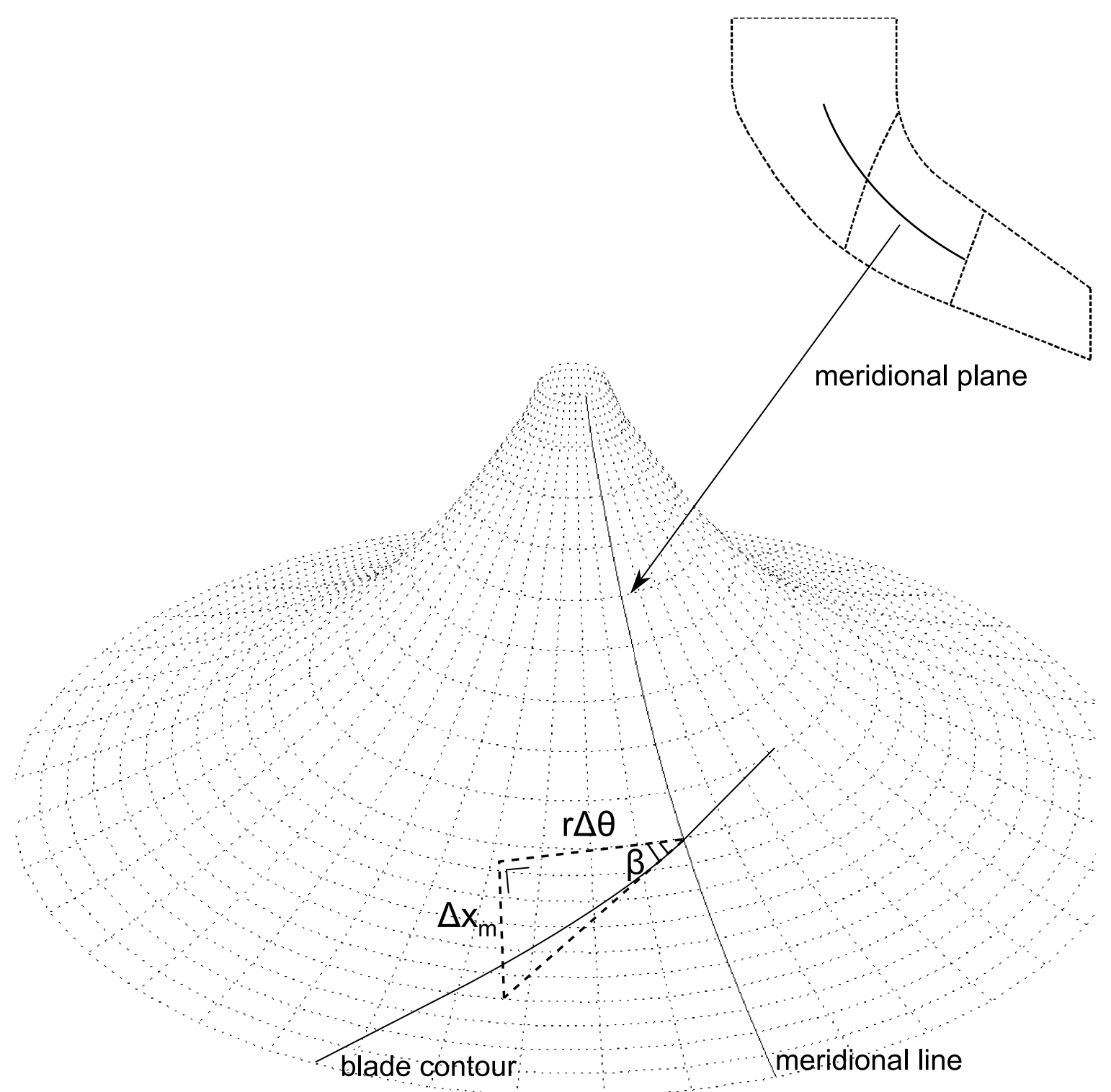

Figure 1.5: Definition of the blade angle $\beta$. The surface of revolution of a meridional line is shown by the dotted lines. The meridional line is also shown in the meridional plane (topright). The blade contour is an intersection of the blade surface with this surface of revolution. The blade angle is defined as the angle of the blade contour with respect to the circumferential direction. 


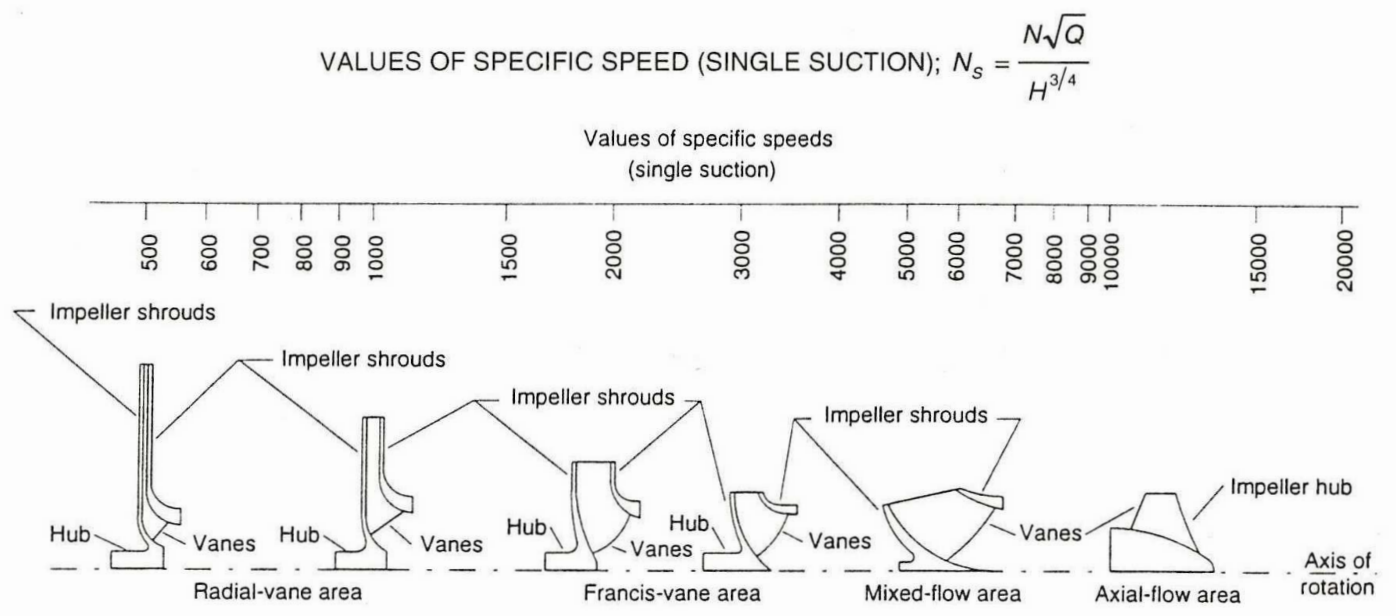

Figure 1.6: Impeller shapes and associated specific speeds, taken from [42]. Here $\mathrm{N}$ is the rotational speed of the pump in revolutions per minute (rpm), Q the flow rate in liters per minute and $\mathrm{H}$ the pump head in meters.

coefficients, another dimensionless number can be formulated that only contains the parameters determining the duty $Q, \Omega, H$. This dimensionless number is the specific speed $N_{s}$

$$
N_{s}=\frac{\Omega \sqrt{Q}}{(g H)^{\frac{3}{4}}}
$$

The specific speed also reflects the impeller shape. For increasing specific speeds the impellers that are used shift from radial, via mixed-flow, to axial impellers, as is depicted in Fig. 1.6. Note that a slightly different definition of the specific speed is used, i.e. the gravitational constant $g$ is not included in the definition, and also different units are used in this figure.

The dimensionless cavitation inception number $\kappa_{i}$ is defined in a similar fashion as the head coefficient

$$
\kappa_{i}=\frac{g \mathrm{NPSH}_{i n c}}{\Omega^{2} D^{2}}
$$

An important parameter to describe the type of flow in the pump is the Reynolds number. For the Reynolds number, the diameter $D$ of the impeller is frequently employed as the characteristic length scale and for the velocity the blade velocity at the trailing edge $u_{t e}=\frac{1}{2} \Omega D$ is taken, resulting in

$$
R e=\frac{\Omega D^{2}}{2 \nu}
$$

where $\nu$ is the kinematic viscosity of the fluid. In pumps the Reynolds number is typically in the order of $10^{6}-10^{7}$, which indicates that the flow inside the boundary layers will be turbulent, except in a region very close to the leading edge. 


\subsection{Basic pump analysis}

The relation between $Q, \Omega$ and $H$ can be clarified by considering a $1 \mathrm{D}$ flow model. Such a model, based on the Euler pump equation, is presented in this section. The 1D theory is a frequently-used method for estimating the performance of (radial) pumps.

Since the impeller rotates around an axis, it is often convenient to work in a rotating frame of reference. For this purpose the relative velocity $\boldsymbol{w}$ is considered, which is defined as the difference between the absolute velocity $\boldsymbol{v}$ and the blade velocity $\boldsymbol{u}=\boldsymbol{\Omega} \times \boldsymbol{r}$, which is in circumferential direction.

$$
\boldsymbol{w}=\boldsymbol{v}-\boldsymbol{u}=\boldsymbol{v}-\Omega \times \boldsymbol{r}
$$

where $\Omega$ is the angular speed vector of the impeller, and $\boldsymbol{r}$ the position vector relative to the origin of the coordinate system, positioned on the axis of rotation. Note that for inviscid flow the relative velocity $\boldsymbol{w}$ is tangential to the blade, since the blade is an impenetrable body, whereas for viscous flow the relative velocity at the blade surface is given by the no-slip condition, $\boldsymbol{w}=\mathbf{0}$. The definition of the relative velocity leads to the velocity triangle, as shown in Fig 1.7.

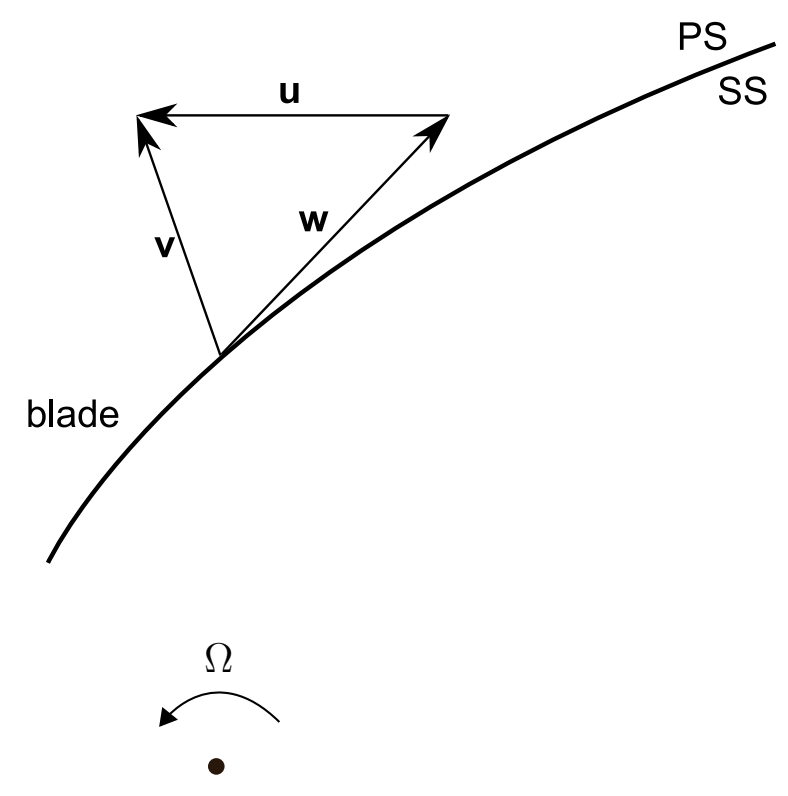

Figure 1.7: The velocity triangle, with $\boldsymbol{v}$ the absolute velocity, $\boldsymbol{w}$ the relative velocity and $\boldsymbol{u}$ the blade velocity. PS indicates the pressure side and SS the suction side of the blade.

To analyze pump performance, the Euler pump equation and velocity triangles are employed. Firstly, the flow is assumed to be steady in the rotating frame. Secondly, the approach assumes a uniform velocity profile from blade to blade, as is illustrated in Fig. 1.8. The analysis is presented here for radial pumps. The Euler pump equation is given by (see for example [26])

$$
W=g H=u_{t e} v_{\theta, t e}-u_{l e} v_{\theta, l e}
$$

where $H$ is the inviscid-flow pump head and $W$ is the energy transfer per unit mass between rotor and fluid. Furthermore, $u$ denotes the (azimuthal) velocity of the blade 
and $v_{\theta}$ the azimuthal component of the absolute flow velocity. The subscripts le and te indicate the leading and trailing edge, respectively. If the fluid enters the pump without pre-rotation $\left(v_{\theta, l e}=0\right)$, the second term on the right side of Eqn. (1.13) cancels out and by substituting $u_{t e}=\Omega r_{t e}$ the Euler relation becomes
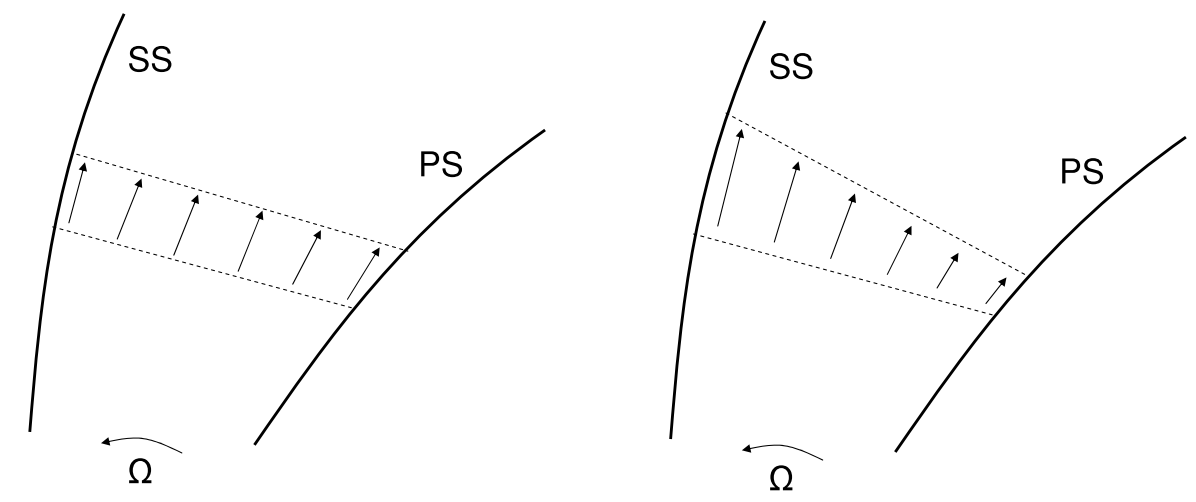

Figure 1.8: Profile of relative velocity in a radial pump channel for the $1 \mathrm{D}$ consideration (left) and the 2D inviscid situation (right). PS indicates the pressure side and SS the suction side of the blades.

$$
W=g H=\Omega r_{t e} v_{\theta, t e}
$$

The $1 \mathrm{D}$ assumption implies that the flow is aligned to the blade, i.e. the flow direction is everywhere equal to the tangent to the blade. Therefore, $v_{\theta}$ can be derived from a velocity triangle as is sketched in Fig. 1.9.

$$
v_{\theta, t e}=u_{t e}-\frac{v_{r, t e}}{\tan \beta_{t e}}
$$

where $\beta_{t e}$ is the blade angle of the impeller at the trailing edge, as defined in section 1.1.4 and $v_{r, t e}$ is the radial component of the absolute velocity (which equals the radial component of the relative velocity $\left.w_{r, t e}\right)$. For a two-dimensional radial pump, assuming uniform flow from pressure to suction side, the flow rate can be computed by

$$
Q=2 \pi r_{t e} b_{t e} v_{r, t e}
$$

where $b_{t e}$ is the width of the impeller at the trailing edge, i.e. the distance from hub to shroud. By substitution of Eqns. (1.15) and (1.16) in Eqn. (1.14), a relationship for the 1D Euler head $H$ can be obtained, showing its dependence on the flow rate $Q$, the angular speed $\Omega$, and the impeller geometry, i.e. $b_{t e}, r_{t e}$ and $\beta_{t e}$.

$$
g H=\Omega r_{t e}\left(\Omega r_{t e}-\frac{Q}{2 \pi r_{t e} b_{t e} \tan \beta_{t e}}\right)
$$




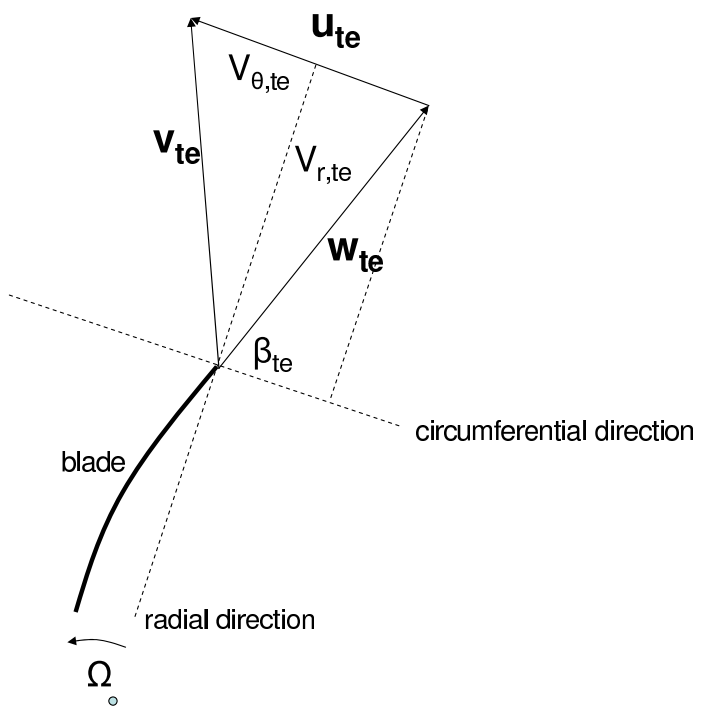

Figure 1.9: Velocity triangle at the trailing edge of a radial pump impeller.

This relationship can also be written in dimensionless form, by considering the definition of the head coefficient $\psi$ and the flow coefficient $\phi$

$$
\psi=\frac{1}{4}\left[1-\phi \frac{4}{\pi \frac{b_{t e}}{r_{t e}} \tan \beta_{t e}}\right]
$$

For backward curved blades, i.e. $0^{\circ}<\beta<90^{\circ}$, the pump head will decrease with increasing flow rate. In reality the velocity distribution at the pump outlet is not uniform, as sketched in Fig. 1.8. The flow experiences a certain 'slip', resulting in a lower value for $v_{\theta, t e}$ and hence a lower value for the pump head $H$ than predicted by the $1 \mathrm{D}$ assumption. If the number of blades for an impeller is increased, this slip is reduced and the head will be closer to the 1D Euler head of the pump. Therefore, the 1D Euler head can be viewed as the head produced by an impeller with an infinite number of blades, where the flow is inviscid. The advantage of the 1D Euler analysis is that it shows the relationship between pump performance and relevant quantities: $\psi=f(\phi$, geometry). 


\subsection{Objective and outline}

The design of centrifugal pump impellers is a highly complicated task due to the complex three-dimensional shape of impeller blades, hub and shroud. Small differences in geometry can lead to significant changes in the performance of such a machine. Advances in computational power over the last few decades have resulted in the application of advanced CFD analysis methods to the prediction of the performance of turbomachines. These methods range from potential flow methods, to fully viscous flow methods.

In this thesis computational methods are developed which can assist the engineer in the design of impellers. The task of the hydraulic engineer is to design a machine which meets the head requirements for a given flow rate with a maximum efficiency and long lifespan. The occurring flow fields determine the hydraulic losses that occur in a pump and also whether cavitation might occur, depending on the inlet pressure of the pump. The occurrence of cavitation influences not only the performance of the machine, but also its lifespan.

In chapter 2 the equations governing the flow inside centrifugal pumps are derived. In this chapter it is argued that for flow conditions near the design point, an inviscid flow model and more specifically a potential flow model can be employed. The derivation of the potential flow model is presented and the employed numerical method, a Finite Element Method, for solving the potential flow equations is discussed.

In this thesis two types of CFD design methods are presented, namely an inversedesign method and an optimization method. The inverse-design method for centrifugal impeller blades is treated in chapter 3. For an inverse-design method the performance of a machine is prescribed and both the flow field and the blade geometry are obtained as a result.

An optimization method for centrifugal impellers is formulated in chapter 4. In such a method the impeller geometry is parameterized and its performance, determined by the flow field, is quantified by a cost function which is to be minimized. The developed method is applied to the design of a radial machine.

This optimized impeller is scaled using the dimensionless numbers discussed in section 1.1.5 and manufactured for use in experiments, which are presented in chapter 5. Particle Image Velocimetry (PIV) is employed to validate the computed velocity profiles for the optimized impeller and to gain more insight in the occurring flow fields inside the impeller. To this end a new experimental setup has been designed and realized. The results of the measurements are compared to the computed results, which have been used in the optimization method.

The results of the preceding chapters are discussed in chapter 6 . Here a critical view is given on the obtained results and recommendations for future research are formulated. 



\section{CHAPTER 2}

\section{Potential Flow Model}

The flow field inside turbomachines influences all performance parameters like head, efficiency and the occurrence of cavitation. Therefore it is eminent that the flow inside the pump is to be modeled accurately. For the inverse-design and optimization methods presented in subsequent chapters a hydrodynamical model is needed. In chapter 1 the 1D theory was presented, for which the flow is assumed to be uniform from blade to blade and from hub to shroud. In this chapter the governing equations for fluid flow in three dimensions are formulated.

In the formulation the simplification is made that the flow can be described by using an incompressible potential flow model. This is an inviscid flow model and in the next section it will be shown under which conditions the potential flow model can be derived from the incompressible Navier-Stokes equations.

\section{$2.1 \quad$ Flow model}

In this section the potential flow equations are derived. The equations are considered in the absolute frame of reference first, i.e. a frame of reference that is stationary in space. Subsequently, the equations are formulated in the rotating frame of reference, i.e. a frame of reference rotating with the impeller blade speed, leading to a steady flow model. The resulting relative velocity field is that seen by an observer rotating with the impeller.

\subsubsection{Absolute frame of reference}

The starting point for the present flow model is formed by the continuity equation (2.1) and the Reynolds-averaged Navier-Stokes equations (2.2) for an incompressible Newtonian fluid with constant viscosity. In an absolute frame of reference they are given by

$$
\boldsymbol{\nabla} \cdot \boldsymbol{v}=0
$$




$$
\rho \frac{\partial \boldsymbol{v}}{\partial t}+\rho \boldsymbol{v} \cdot \nabla \boldsymbol{v}=-\nabla p+\eta \nabla^{2} \boldsymbol{v}+\boldsymbol{\nabla} \cdot \boldsymbol{R}+\rho \boldsymbol{g}
$$

where $\rho$ is the density, $\boldsymbol{v}$ the absolute velocity, $\eta$ the dynamic viscosity, $p$ the pressure, $\boldsymbol{R}$ the turbulent Reynolds stresses and $\boldsymbol{g}$ the gravitational acceleration. The Reynolds stresses are given by $\boldsymbol{R}=-\rho \overline{\boldsymbol{v}^{\prime} \boldsymbol{v}^{\prime}}$, where $\boldsymbol{v}^{\prime}$ indicates the velocity fluctuation and the over-bar indicates time-averaging.

In Eqns. (2.1) and (2.2) the flow is assumed to be incompressible. This assumption is justified when the following condition is met

$$
M a^{2}=\left(\frac{v^{*}}{a}\right)^{2}<<1
$$

where $v^{*}$ is the magnitude of a reference velocity, $M a$ is the Mach number and $a$ the speed of sound.

When $R e$ is large $(R e>>1)$, as is the case in most turbomachinery flows, the viscous term $\eta \nabla^{2} \boldsymbol{v}$ in Eqn. (2.2) can be neglected outside boundary layers and wakes. Furthermore, the turbulence intensity $T u$ is defined as the ratio between the velocity fluctuation $v^{\prime}$ and the mean velocity $v$

$$
T u=\frac{\left|v^{\prime}\right|}{|v|}
$$

In the core flow, outside the boundary layers, the turbulence intensity is low: $T u<<1$, meaning that the Reynolds stresses $\rho \overline{\boldsymbol{v}^{\prime} \boldsymbol{v}^{\prime}}$ can be neglected [31]. Thus the Euler equations are obtained

$$
\rho \frac{\partial \boldsymbol{v}}{\partial t}+\rho \boldsymbol{v} \cdot \boldsymbol{\nabla} \boldsymbol{v}=-\boldsymbol{\nabla} p+\rho \boldsymbol{g}
$$

When viscous effects are neglected, as in Eqn. (2.5), the flow is called inviscid. This equation is rewritten, using the vector identity:

$$
\boldsymbol{v} \cdot \boldsymbol{\nabla} \boldsymbol{v}=\frac{1}{2} \boldsymbol{\nabla}(\boldsymbol{v} \cdot \boldsymbol{v})+(\boldsymbol{\nabla} \times \boldsymbol{v}) \times \boldsymbol{v}
$$

Combining Eqn. (2.5) and Eqn. (2.6) gives

$$
\frac{\partial \boldsymbol{v}}{\partial t}+\frac{1}{2} \nabla(\boldsymbol{v} \cdot \boldsymbol{v})+(\boldsymbol{\nabla} \times \boldsymbol{v}) \times \boldsymbol{v}=-\frac{1}{\rho} \nabla p+\boldsymbol{g}
$$

According to Kelvin's circulation theorem, in an inviscid barotropic fluid subjected to a conservative force field the circulation of any closed curve moving with the flow field remains constant. This means that if the incoming flow is irrotational, the flow remains irrotational everywhere in the domain considered. The flow is said to be irrotational when

$$
\nabla \times v=0
$$

For irrotational flow a velocity-potential $\phi$ can be defined such that Eqn. (2.8) is satisfied automatically

$$
\boldsymbol{v}=\nabla \phi
$$


The continuity equation (2.1) and the Euler equation (2.7) now reduce further to

$$
\begin{aligned}
\nabla^{2} \phi & =0 \\
\frac{\partial \boldsymbol{\nabla} \phi}{\partial t}+\frac{1}{2} \boldsymbol{\nabla}(\boldsymbol{v} \cdot \boldsymbol{v}) & =-\frac{1}{\rho} \boldsymbol{\nabla} p+\boldsymbol{g}
\end{aligned}
$$

Equation (2.10) is the well-known Laplace equation for fluid flow. From Eqn. (2.11) the Bernoulli equation for unsteady incompressible potential flow is obtained

$$
\frac{\partial \phi}{\partial t}+\frac{1}{2} \boldsymbol{v} \cdot \boldsymbol{v}+\frac{p}{\rho}+\boldsymbol{g} \cdot \boldsymbol{r}=c(t)
$$

where $\boldsymbol{r}$ is the position vector.

The boundary-layer thickness $\delta$ for the turbulent boundary layer on a flat plate, with a uniform velocity outside the boundary layer, can be estimated for $5 \cdot 10^{5}<R e_{x}<10^{7}$ by (see for example [58])

$$
\delta(x)=0.37 x R e_{x}^{-0.2}
$$

where $x$ is the distance from the starting point of the boundary layer. For a Reynolds number of $10^{7}$, typically encountered in turbomachines, this implies a boundary layer thickness of $\delta / x=0.023$. This indicates that for most turbomachinery applications the boundary layer is sufficiently thin, so for attached flows the displacement effect of the boundary layer on the core flow is neglected here.

\subsubsection{Rotating frame of reference}

In this section the transformation is made to a rotating frame of reference, i.e. a frame which rotates with the impeller blade speed. The equations are to be formulated in terms of the relative velocity $\boldsymbol{w}$, as has been discussed in section 1.2. This relative velocity is thus the velocity as seen by an observer rotating with the impeller.

The material derivative of a scalar quantity is objective, hence

$$
\frac{\partial \phi}{\partial t}+\boldsymbol{v} \cdot \boldsymbol{\nabla} \phi=\frac{D \phi}{D t}=\left.\frac{D \phi}{D t}\right|_{R}=\left.\frac{\partial \phi}{\partial t}\right|_{R}+\boldsymbol{w} \cdot \boldsymbol{\nabla} \phi
$$

where $\left.\frac{\partial \phi}{\partial t}\right|_{R}$ is the time derivative in the rotating frame. Substitution of Eqns. (2.14) and (1.12) in Eqn. (2.12) gives the following equation (in the rotating frame of reference) after some algebra

$$
\left.\frac{\partial \phi}{\partial t}\right|_{R}+\frac{p}{\rho}+\frac{1}{2}|\boldsymbol{w}|^{2}-\frac{1}{2}|\boldsymbol{\Omega} \times \boldsymbol{r}|^{2}+\boldsymbol{g} \cdot \boldsymbol{r}=c(t)
$$

The free impeller case is considered. This implies that the flow inside the stationary parts of the diffuser does not influence the flow inside the rotating parts of the impeller and that the incoming flow is rotationally symmetric. Furthermore, all blades are assumed to be equal in shape and equally spaced. These assumptions can be justified for an impeller 
without a diffuser or for an impeller with a well designed vaneless diffuser operating at the Best Efficiency Point (BEP). In the free impeller case the potential-flow field is steady for an observer that rotates with the impeller, i.e. $\left.\frac{\partial \phi}{\partial t}\right|_{R}=0$. Then a rothalpy $I$ can be defined such that it is constant in the rotating frame of reference

$$
I=\frac{p}{\rho}+\frac{1}{2}|\boldsymbol{w}|^{2}-\frac{1}{2}|\boldsymbol{\Omega} \times \boldsymbol{r}|^{2}+\boldsymbol{g} \cdot \boldsymbol{r}=c(t)
$$

as follows from Eqn. (2.15). This equation is referred to as the Bernoulli equation in the rotating frame of reference.

Summarizing, the assumptions that are made in incompressible potential-flow theory are

- Inviscid flow: Re >> 1; boundary layer separation does not occur; low turbulence intensity.

- Incompressible flow: $M a^{2}<<1$

- Irrotational flow: $\boldsymbol{\nabla} \times \boldsymbol{v}=\mathbf{0}$ at the inlet

For centrifugal pumps or fans these assumptions are quite reasonable when they are operating near the design point. By solving Eqn. (2.10) the velocity field is obtained, and the pressure is calculated by using Eqn. (2.16).

Furthermore, it has to be mentioned that usually relative velocity profiles are considered in this thesis. Since the absolute velocity field is irrotational, i.e. $\boldsymbol{\nabla} \times \boldsymbol{v}=\mathbf{0}$, this means that the relative velocity field is not irrotational

$$
\boldsymbol{\nabla} \times \boldsymbol{w}=\boldsymbol{\nabla} \times(\boldsymbol{v}-\boldsymbol{\Omega} \times \boldsymbol{r})=-\boldsymbol{\nabla} \times(\boldsymbol{\Omega} \times \boldsymbol{r})=-2 \boldsymbol{\Omega}
$$

as follows from Eqn. (1.12). This means that the model is capable of predicting back-flow (also called reverse-flow) in the impeller, as is sketched in Fig. 2.1. Back-flow can occur for low flow rates and is undesirable since it is known to lead to unstable operation in the field, although in practice many impellers are known to have a back-flow region, even when operating at design conditions. Note that when back-flow is predicted, the validity of the potential flow model is lost, since boundary layer separation will have occurred in reality.

When the free impeller case is considered, it suffices to consider a single impeller blade channel only. Such a domain is sketched in Fig. 2.2. Boundary conditions for the Laplace equation need to be formulated on this domain. This is discussed in the next section.

\subsection{Boundary conditions}

In order to solve the Laplace equation (2.10), boundary conditions need to be formulated at the boundary of the domain of interest. Assuming periodicity of the flow field, i.e. when all blades are identical in shape and equally spaced from each other (free impeller 


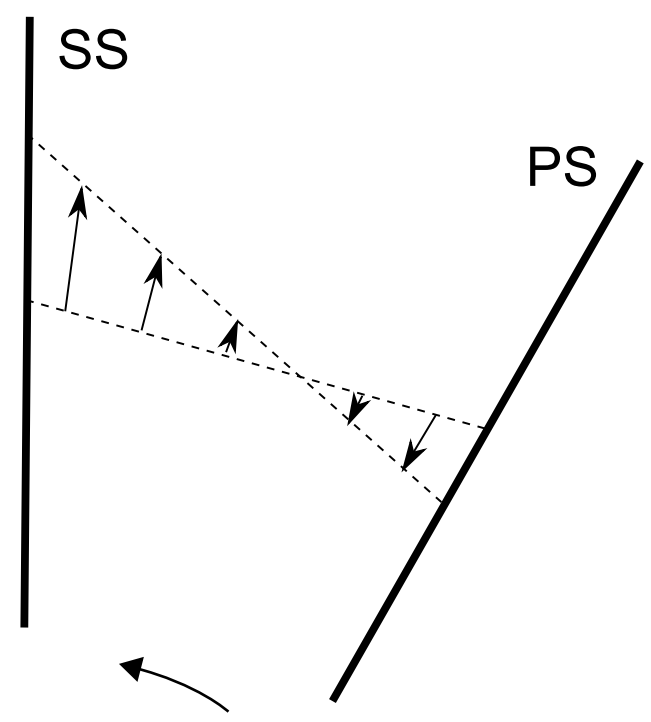

Figure 2.1: $\quad$ Sketch of an inviscid relative velocity profile in an impeller with straight blades with back-flow occurring at the pressure side. PS indicates the pressure and SS the suction side, respectively.

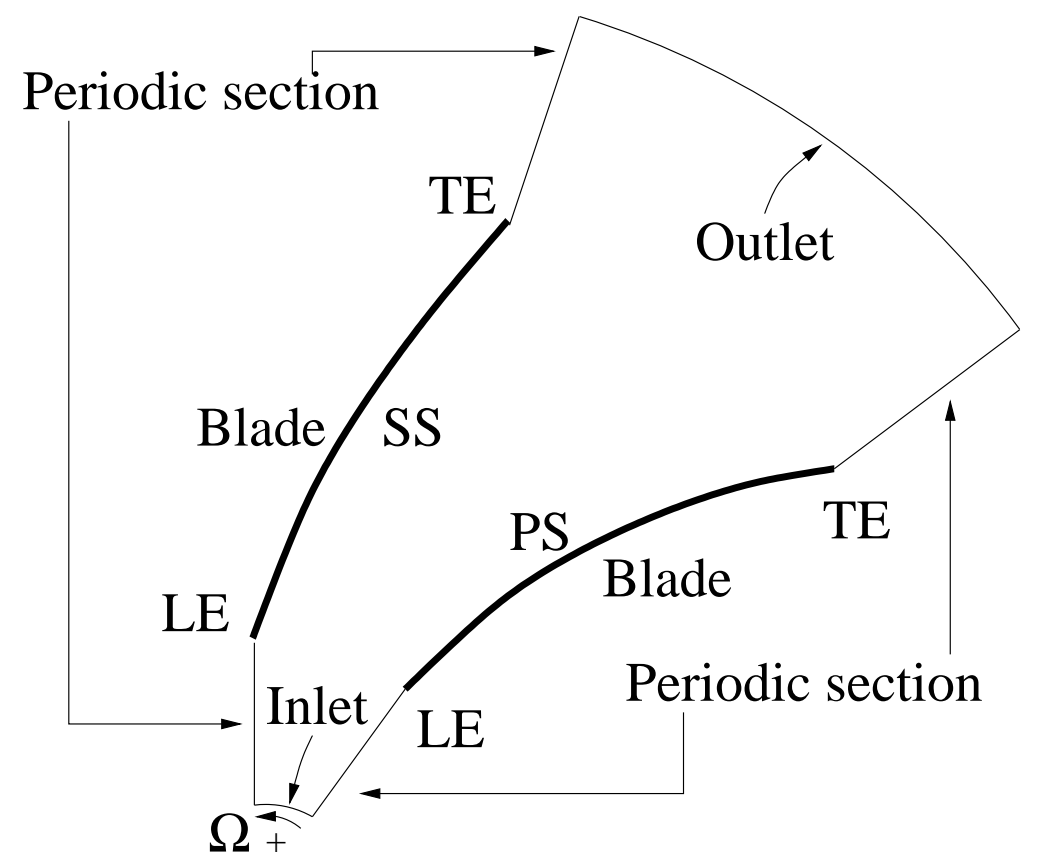

Figure 2.2: $\quad$ Flow domain of interest between two blades. The hub is above and the shroud below this plane. PS is the pressure side and SS the suction side, LE the leading edge and TE the trailing edge of the blade. 
case), only a single impeller channel needs to be considered. This impeller channel is sketched in Fig. 2.2.

For an incoming flow without pre-swirl, a Dirichlet or essential boundary condition applies at the circular inlet of the domain

$$
\phi=0
$$

At the outlet of the domain, sufficiently far away from the trailing edge, the flow is assumed to be uniform and thus a Neumann boundary condition is formulated

$$
\frac{\partial \phi}{\partial n}=v_{n}=\frac{Q}{Z A_{\text {out }}}
$$

where $A_{\text {out }}$ is the surface area of the outlet section and $Z$ the number of blades on the impeller.

The hub and the shroud are impenetrable surfaces of revolution. Thus the following Neumann boundary condition holds

$$
w_{n}=v_{n}=\frac{\partial \phi}{\partial n}=0
$$

Since the flow field within each channel formed by the two blades is identical, it follows that for the periodic sections upstream and downstream of the blade (see Fig. 2.2) the following must hold

$$
\boldsymbol{v}\left(r, \theta_{p s}\right)=\boldsymbol{R} \boldsymbol{v}\left(r, \theta_{s s}\right)
$$

where $p s$ and $s s$ indicate the pressure and suction side of the domain, respectively. $\boldsymbol{R}$ is the rotation matrix for rotation around the $z$-axis over an angle of $-2 \pi / Z$. Alternatively, the normal components and in plain components of the velocity at the pressure side and suction side are equal. The corresponding boundary conditions for the velocity potential $\phi$ are given by

$$
\begin{gathered}
\frac{\partial \phi}{\partial n}\left(r, \theta_{p s}\right)=-\frac{\partial \phi}{\partial n}\left(r, \theta_{s s}\right) \\
\phi\left(r, \theta_{p s}\right)=\phi\left(r, \theta_{s s}\right)+c
\end{gathered}
$$

Upstream of the blade surface $c=0$, due to the assumption of incoming flow without pre-swirl, and downstream the value will be determined by the circulation $\Gamma$ generated by the impeller $(c=\Gamma)$.

The pressure and suction side of the blades are impenetrable. Thus the impenetrability or blade stream-surface condition applies

$$
\boldsymbol{w} \cdot \boldsymbol{n}=0
$$

where $\boldsymbol{n}$ is the outward normal vector at the blade surface. By making use of Eqn. (1.12) this condition can also be written as

$$
v_{n}=\frac{\partial \phi}{\partial n}=u_{n}=(\boldsymbol{\Omega} \times \boldsymbol{r}) \cdot \boldsymbol{n}
$$


At the trailing edge the the flow is tangential to the blade, which is the so-called Kutta condition. Several approaches can be utilized to impose the Kutta condition (see for example [14]). For the direct method, used in the optimization method presented in chapter 4, the employed Kutta condition is that the velocity just downstream of the trailing edge is parallel to the blade, i.e.

$$
v_{n, t e}=u_{n, t e}
$$

where $u_{n, t e}$ is the blade speed at the trailing edge. For the inverse-design method discussed in Chapter 3 the Kutta condition will be enforced via the prescribed mean-swirl distribution.

\subsection{Augmented potential flow model}

In the preceding sections the potential flow model with associated boundary conditions has been presented. This model is an inviscid flow model and can not be utilized to determine hydraulic losses inside turbomachines directly. However, the model can be extended to the so-called augmented potential flow model, by adding loss models to the potential flow model. If the boundary layers are thin and flow separation does not occur, the boundary layer losses in the power, $\Delta P_{\text {loss }}$, can be estimated by (see [24])

$$
\Delta P_{\text {loss }}=\int_{S} C_{D} \frac{1}{2} \rho w^{3} d S
$$

where $C_{D}$ is the energy dissipation coefficient, estimated at 0.0038 [24] and $S$ is the surface area of the boundary considered. By using this approach the losses can be quantified.

The boundary layer losses in the impeller are only a part of the total losses occurring. Firstly, there are also boundary layer losses occurring in the volute, but these are not considered here, since the focus is on the design of impellers. Furthermore, leakage losses, mixing losses and disc friction losses also lead to a reduction in efficiency. These extra losses can be taken into account in the model as well, as is done elsewhere [32], but they are also largely dependent on the specific speed $N_{s}$ as is shown for example in [63]. Since the specific speed of the impellers in this thesis are not altered, these hydraulic losses occurring in the impeller are not considered, and only the boundary layer losses in the impeller are taken into account.

The loss coefficient $\zeta$ is defined as the ratio between the boundary layer losses and the hydraulic power of the machine $P_{H}$, which is given by Eqn. (1.3).

$$
\zeta=\frac{\Delta P_{l o s s}}{P_{H}}
$$

One of the aims in pump impeller design obviously is to obtain an impeller with a low loss coefficient $\zeta$. 


\subsection{Numerical method}

For the incompressible potential flow model the equation to be solved is the Laplace equation (2.10) with appropriate boundary conditions. By solving this equation the velocity potential $\phi$ becomes known. Then the velocity $\boldsymbol{v}$ can be determined from Eqn. (2.9) and the static pressure follows from Eqn. (2.16).

This section is devoted to the numerical method employed for solving the Laplace equation. The adopted Finite Element Method (FEM) approach is based on the discretization of the weak form of the Laplace equation.

\subsubsection{Weak form of the Laplace equation}

The weak form of the Laplace equation (2.10) is derived here. It is obtained by multiplying the Laplace equation by a test function $\psi$ and integrating over the domain $V$.

$$
\int_{V}\left(\psi \nabla^{2} \phi\right) d V=0
$$

Using Gauss' theorem, it follows that

$$
\int_{V} \nabla \psi \cdot \nabla \phi d V=\int_{S} \frac{\partial \phi}{\partial n} \psi d S
$$

The boundary of the domain consists of a Dirichlet (or essential) boundary surface, $S_{D}$, a Neumann boundary, $S_{N}$, and periodic boundaries, $S^{+}$and $S^{-}$(see section 2.2). The test function $\psi$ must vanish on the Dirichlet boundary and on periodic boundaries it must satisfy $\psi^{+}=\psi^{-}$. Hence, it follows that

$$
\int_{V} \nabla \psi \cdot \nabla \phi d V=\int_{S_{N}} v_{n} \psi d S+\int_{S^{+}} v_{n} \psi d S+\int_{S^{-}} v_{n} \psi d S
$$

where $v_{n}$ is the prescribed value of the absolute velocity normal to the surface under consideration.

The boundary surfaces at which a Neumann boundary conditions applies, $S_{N}$, consist of the outlet section, $S_{\text {out }}$, the hub, $S_{h u b}$, the shroud, $S_{s h r}$, the blade pressure side, $S_{P S}$, and the blade suction side, $S_{S S}$, see also Fig. 2.2. The contribution of the hub and the shroud to the right hand side of Eqn. (2.31) is zero, since $(\partial \phi / \partial n)=0$, see Eqn. (2.20).

The periodic boundary condition given in Eqn. $(2.21)$, i.e. $(\partial \phi / \partial n)^{+}=-(\partial \phi / \partial n)^{-}$, and the condition for the test function, $\psi^{+}=\psi^{-}$, imply that the second and third term of the right hand side cancel, resulting in

$$
\int_{V} \nabla \psi \cdot \nabla \phi d V=\int_{S_{N}} v_{n} \psi d S
$$




\subsubsection{Structured mesh generation}

For both the direct and inverse method tetrahedral meshes are employed. These tetrahedral meshes are the meshes on which the finite element method is applied. The generation of the mesh starts by dividing the domain into a structured mesh of hexahedrons, each with eight nodes. Each hexahedron is subsequently divided in six tetrahedra, as sketched in Fig. 2.3. In this figure it is also shown how, starting from a 2D mesh of quadrilaterals (analogous to hexahedrons in 3D), a mesh of triangles (analogous to tetrahedrons in 3D) is obtained. The diagonal of each quadrilateral is chosen such that a mesh of triangles with good quality is obtained. Mesh refinement can also be applied. This is generally
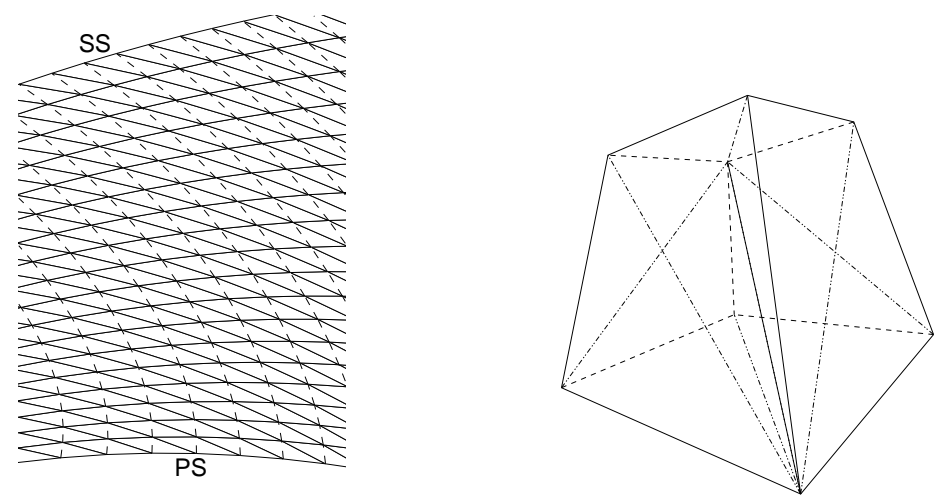

Figure 2.3: The division of a $2 \mathrm{D}$ quadrilateral mesh into a triangular mesh (left) and the division of a 3D hexahedron into six tetrahedra.

employed near boundary surfaces of interest.

\subsubsection{Finite Element Method}

In this section the weak form of the Laplace equation (2.32) is discretized using the Finite Element Method (FEM). The volume $V$ forming the computational domain is divided into volume elements. The potential $\phi(\boldsymbol{x})$ is described by using basis functions $N^{j}(\boldsymbol{x})$ corresponding to the finite element mesh. The weight functions $\psi$ are taken equal to the basis functions, i.e. the Galerkin method is employed.

$$
\begin{aligned}
\phi(\boldsymbol{x}) & =\sum_{j=1}^{n} \phi^{j} N^{j}(\boldsymbol{x}) \\
\psi(\boldsymbol{x}) & =N^{i}(\boldsymbol{x}) \quad i=1 \ldots n
\end{aligned}
$$

where $n$ is the number of nodes in the domain and $\boldsymbol{x}^{i}$ are the nodes in the domain. Since $N^{j}\left(\boldsymbol{x}^{i}\right)=\delta_{i j}$, the $\phi^{j}$-values correspond to the unknown nodal values. The discretized equations become

$$
\left\{\sum_{j=1}^{n} \int_{V} \boldsymbol{\nabla} N^{i} \cdot \nabla N^{j} d V\right\} \phi^{j}=\int_{S_{N}} v_{n} N^{i} d S
$$


This is a linear system of equations for $\phi^{j}$. Linear basis functions are employed here, leading to a second-order accuracy for the velocity potential.

\subsubsection{Determination of velocity}

In the preceding section the numerical method for computing the velocity potential in the mesh nodes has been presented. In each tetrahedral centroid the velocity is determined by using the velocity potential $\phi^{i}$ at the four nodes of the tetrahedron.

$$
\boldsymbol{v}^{c}=\boldsymbol{\nabla} \phi^{c}=\sum_{i=1}^{4} \phi^{i} \nabla N^{i}
$$

where $c$ denotes the centroid of a tetrahedron. The velocity potential $\phi(\boldsymbol{x})$ is continuous for linear basis functions $N^{i}$. However, the velocity $\boldsymbol{v}=\boldsymbol{\nabla} \phi$ is discontinuous over element edges, since $\boldsymbol{\nabla} N^{i}$ is constant inside the elements, and discontinuous at the element edges. This is illustrated for a 1D mesh in Fig. 2.4.
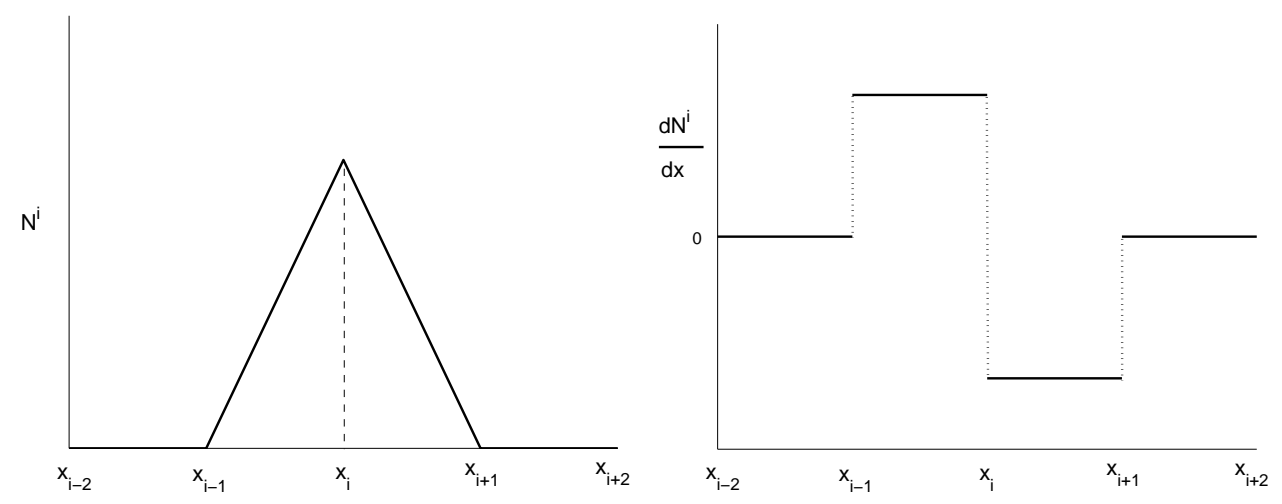

Figure 2.4: Linear basis function $N^{i}(x)$ (left) and its derivative $\frac{d N^{i}}{d x}$ (right) on a 1D mesh.

To obtain values for $\boldsymbol{v}$ in the nodal points, the Superconvergent Patch Recovery (SPR) method is utilized (Zienkiewicz et al. [84]). For this purpose patches are constructed.

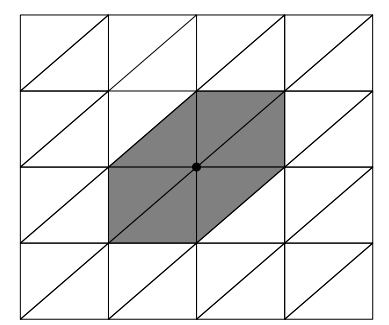

Figure 2.5: 2D patch used for velocity reconstruction. 
The patch for a node consists of those elements to which this nodes belongs. A twodimensional sketch of such a patch (thus with triangles instead of tetrahedrons) is given in Fig. 2.5. The velocity in a nodal point $\boldsymbol{v}^{i}$ is determined by a linear least-squares fit, i.e. $\boldsymbol{v}=\boldsymbol{a}+\boldsymbol{b} x+\boldsymbol{c} y+\boldsymbol{d} z$, using the data points $\boldsymbol{v}^{c}$ taken from the patch for node $i$.

The SPR method is very accurate for the internal domain, where large, more or less, symmetrical patches are constructed. The second-order accuracy for the potential is retained in a second-order accuracy for the velocity [31]. However, the accuracy of the SPR method decreases near boundaries, where smaller patches are considered. This will generally result in a lower order of accuracy for the velocity near boundaries. 



\section{CHAPTER 3}

\section{Inverse-design Method}

The design and analysis of turbomachines is a complex task due to the involved threedimensional shapes, for example of the impeller blades. The application of CFD software to evaluate the performance of a specified geometry is frequently designated as a direct method. The performance characteristics, like pump head and pressure distribution, are obtained as a result of this direct flow analysis.

For design purposes it is often desirable to solve the inverse problem. In such an inverse-design method the performance characteristics are prescribed by some performance function, the so-called loading distribution, and the corresponding geometry is obtained as a result of an inverse analysis. Both flow field and geometry are obtained from this procedure.

In this chapter such an inverse-design method for centrifugal impeller blades is presented. A literature overview of inverse-design methods is given in section 3.1, with the focus on turbomachinery applications. Next, the developed inverse-design method is discussed in section 3.2. The numerical implementation is treated in section 3.3. The developed method is verified in section 3.4, where also the order of accuracy of the method is determined. Inverse-design cases are considered in section 3.5 and 3.6, for a radial and a mixed-flow impeller, respectively. Alternative loading distributions are presented in section 3.7. Finally, in section 3.8 the developed method is discussed and conclusions are drawn.

\subsection{Literature overview}

In this section an overview is given of available literature on inverse-design methods, with the focus on turbomachinery applications. Inverse-design methods are frequently used in the design of airfoils. In the 1980s and 1990s several programs were developed that can be used for inverse airfoil design, most notably Xfoil [27] and Profil [30], which are available on the internet. Both of these codes are based on a panel method for two-dimensional 
incompressible potential flow, with incorporated inverse-design methods. Since then more sophisticated Navier-Stokes methods have been developed.

Inverse-design methods for turbomachinery impellers originate from Werner von Braun's group of scientists, which was responsible for designing the V-1 and V-2 rockets in World War II. Hans Spring [61] reports on attending a lecture by a German professor entitled Theory of Impeller Vane Design via Prescribed Averaged Circulation in the 1950s. Since the 1950s, several two-dimensional and later quasi three-dimensional inverse-design methods have been developed. In fact, quasi three-dimensional methods are still being used for example by Peng et al. [51],[52].

The first three-dimensional inverse-design method for turbomachines was outlined in the combined papers by Hawthorne et al. [40] and Tan et al. [66]. A prescribed meanswirl distribution is used to design impeller blades for annular cascades of infinitesimally thin blades. This method was extended by Borges [6] and Zangeneh [80] to radial and mixed-flow turbomachines. In these approaches the potential flow model is employed. Borges applied his method to the design of a low-speed radial-inflow turbine and gives some recommendations on the choice of a suitable mean-swirl distribution. Zangeneh et al. used a derivative of the mean-swirl distribution with the aim of suppressing secondary flows both in a mixed-flow pump impeller [81] and a compressor diffuser [82]. Goto et al. [39] employed a similar approach to the redesign of pump diffuser blades, in order to suppress flow separation. The hub side was more front loaded than the shroud side to achieve this. Moreover, Zangeneh et al. [83] utilized this method to design a centrifugal compressor with splitter blades.

Demeulenaere et al. [22] developed an inverse-design method incorporating a prescribed pressure distribution, instead of a mean-swirl distribution, to design compressor and turbine blades using the Euler model for three-dimensional inviscid flow. Veress et al. [68] used this inverse-design approach in the design of a multistage radial compressor, in order to obtain a smoother Mach number distribution along the blades. The inviscid inverse-design method of Demeulenaere was used by De Vito et al. [70] in combination with a direct two-dimensional Navier-Stokes method in an iterative scheme to re-design a turbine nozzle blade.

Dang et al. developed an inverse method utilizing the Euler model for two-dimensional cascades [19] and later for fully three-dimensional geometries [18]. They utilized a prescribed pressure distribution and a prescribed thickness distribution. Damle et al. [16] used the same approach in order to increase the efficiency of a first-stage rotor in a centrifugal compressor. Jiang et al. [43] employed the method for the design of an inlet guide vane, a turbine blade and a compressor blade.

Peng et al. [50, 51, 52] developed a quasi 3D inverse-design method by circumferential averaging. They employed a stream function for the inviscid flow analysis and used a prescribed mean-swirl distribution as loading function. The method was applied to the design and optimization of a turbine. Cao et al. [10] utilized this quasi 3D approach in combination with a direct flow analysis for the hydrodynamic design of a gas-liquid two-phase flow impeller.

Daneshkhah et al. [17] developed a two-dimensional Navier-Stokes inverse method using a prescribed pressure and thickness distribution. They applied the method to the redesign of a subsonic turbine and a transonic compressor. Wang et al. [71] presented 
a 3D inverse method based on the Navier-Stokes equations, using a prescribed pressure distribution.

In the next sections an inverse-design method is presented based on similar principles as outlined by Borges [6] and Zangeneh [80]. The method is thus also based on a potential flow model. The main difference is that a coupled approach (from hub to shroud) is utilized to find the solution of the inverse-design problem. Due to this coupled approach the method is very robust and capable of dealing with a higher resolution of the flow in the region from blade to blade. Furthermore, a different numerical approach, using the Finite Element Method, is employed. The presented work has also been reported elsewhere [76].

\subsection{Inverse-design method}

In this section the inverse-design problem is formulated, whose solution provides the flow field and the impeller geometry that realizes the specified hydraulic characteristics. Firstly, the design conditions are considered. These are the conditions that are specified before an inverse-design computation can be performed. They describe the desired performance of such an inverse-design. Secondly, a curvilinear coordinate system in the meridional plane is presented in section 3.2.2. One of the design conditions, the meanswirl distribution, is discussed in detail in section 3.2.3. The inverse-design algorithm and the impenetrability condition are treated in subsequent sections. Only cases with infinitesimally small blade thickness are considered.

\subsubsection{Design conditions}

At the start of each inverse-design problem, the design conditions have to be selected. If one of these conditions is altered, a different inversely-designed impeller is obtained. These so-called design conditions are

- $Q$, the flow rate

- $H$, the pump head to be achieved

- $\Omega$, the rotational speed of the impeller

- $(r, z)_{\text {blade }}$, the meridional blade shape, including inlet and outlet sections

- $\delta=0$, blades of zero thickness

- $\theta_{T E}$, the stacking condition at the trailing edge

- $Z$, the number of blades of the impeller

- $r \bar{v}_{\theta}(r, z)$, the mean-swirl distribution

The mean-swirl distribution is employed as performance function. By prescribing the distribution of this quantity in an appropriate way the desired pump head and desirable flow conditions are obtained. In section 3.2.3 the mean-swirl distribution is discussed in 


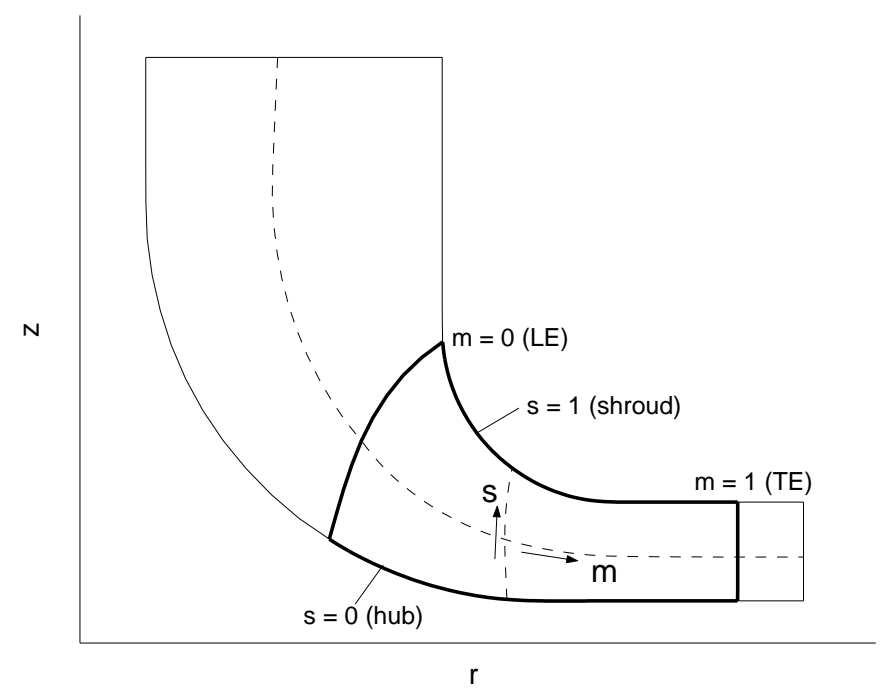

Figure 3.1: Meridional coordinates $m$ and $s$.

more detail. Note that part of the geometry, i.e. the meridional geometry, is prescribed in the inverse-design method. Thus only the unknown blade curvature is to be obtained as a result of the inverse-design procedure.

\subsubsection{Curvilinear coordinate system}

In the preceding section it has been discussed that the meridional geometry of the impeller blades is part of specified design conditions and therefore remains unchanged in the inverse-design method. The mean-swirl distribution, which is discussed in the next section, is prescribed as function of the meridional coordinates $r, z$, i.e. $r \bar{v}_{\theta}(r, z)$.

It is often convenient to use a curvilinear coordinate system $m, s$ in the meridional plane, as is sketched in Fig. 3.1, with $r(m, s)$ and $z(m, s)$. Here $m$ is the normalized length from leading to trailing edge, along the meridional line for which $s$ is constant; $m=0$ corresponds to the leading edge and $m=1$ to the trailing edge of the blade. Similarly, $s$ is the normalized length from hub to shroud in span-wise direction for which $m$ is constant; $s=0$ corresponds to the hub and $s=1$ to the shroud. Note that the $m$ - and $s$-directions do not need to be orthogonal. The main advantage of using such a curvilinear coordinate system is that it can be used for radial, mixed-flow and axial impellers, i.e. for any impeller. Summarizing, the coordinate system is often formulated in terms of $m, \theta, s$ instead of $r, \theta, z$.

\subsubsection{Mean-swirl distribution}

The mean-swirl is defined as the mean of the swirl $r \bar{v}_{\theta}$ (or angular momentum) along a circular arc from pressure to suction side of the blades. Therefore it is a function of the meridional coordinates $r, z$ or of $m, s$ (see section 3.2.2), i.e. $r \bar{v}_{\theta}(m, s)$.

The difference in potential between the pressure and suction side of a blade, $\Delta \phi$, is 
related to the mean-swirl distribution

$$
\begin{aligned}
\Delta \phi(m, s) & \equiv \phi_{s s}-\phi_{p s}=\int_{p s}^{s s} \frac{\partial \phi}{\partial s} d s=\int_{\theta_{p s}}^{\theta_{s s}} v_{\theta}(m, \theta, s) r d \theta=r \int_{\theta_{p s}}^{\theta_{s s}} v_{\theta}(m, \theta, s) d \theta \\
& =\frac{2 \pi}{Z} r \bar{v}_{\theta}(m, s)
\end{aligned}
$$

The integration is performed along a circular $\operatorname{arc}(d s=r d \theta)$ and the blades are assumed to be of infinitesimally small thickness, i.e. $\theta_{s s}-\theta_{p s}=2 \pi / Z$, where $Z$ is the number of blades of the impeller.

In order to design impeller blades with desired flow conditions, requirements need to be formulated for the mean-swirl distribution. An important requirement follows from the assumption that the flow enters the impeller without pre-rotation, hence at the leading edge, $\bar{v}_{\theta}(0, s)=0$, see section 1.2 . Thus

$$
r \bar{v}_{\theta}(0, s)=0
$$

where $r \bar{v}_{\theta}$ is written as function of $m$ and $s$. The Euler pump equation has been given in section 1.2. Here it is utilized, in combination with Eqn. (3.2), to formulate a second requirement for $r \bar{v}_{\theta}(m, s)$, since an impeller is designed for a certain inviscid head $H$

$$
\begin{gathered}
g H=u(1, s) \bar{v}_{\theta}(1, s)-u(0, s) \bar{v}_{\theta}(0, s)=\Omega r(1, s) \bar{v}_{\theta}(1, s) \\
r \bar{v}_{\theta}(1, s)=\frac{g H}{\Omega}
\end{gathered}
$$

where $u(1, s)$ and $u(0, s)$ are the blade speeds at the trailing edge and leading edge, respectively. The ideal-flow head $H$ utilized in Eqn. (3.4) can be obtained from the head using some viscous correction. Note that the slip factor is included in the ideal-flow head.

Furthermore, the pressure difference between pressure side and suction side $p_{p s}-p_{s s}$ is derived from the Bernoulli equation in the rotating frame, Eqn. (2.16), for blades with zero thickness, i.e. $u_{p s}=u_{s s}$

$$
\begin{aligned}
\Delta p \equiv p_{p s}-p_{s s} & =\frac{1}{2} \rho\left(w_{s s}^{2}-w_{p s}^{2}\right)-\frac{1}{2} \rho\left(u_{s s}^{2}-u_{p s}^{2}\right) \\
& =\rho \overline{\boldsymbol{w}} \cdot \nabla\left(\phi_{s s}-\phi_{p s}\right)
\end{aligned}
$$

where $\overline{\boldsymbol{w}}=\frac{1}{2}\left(\boldsymbol{w}_{p s}+\boldsymbol{w}_{s s}\right)$ is the average of the relative velocity on the pressure and suction side.

At the leading and trailing edge the difference in potential $\Delta \phi$ is constant in spanwise direction (see Eqns. (3.2) and (3.4)). Therefore, at the leading and trailing edge $\partial \Delta \phi / \partial s=0$. Furthermore, the impenetrability condition states that $w_{n, s s}=w_{n, p s}=0$. Therefore, the following requirements are found at the leading edge $(m=0)$ and at the trailing edge $(m=1)$

$$
\begin{aligned}
\Delta p(0, s) & =\rho \bar{w}_{l}(0, s) \frac{\partial}{\partial x_{l}} \Delta \phi(0, s) \\
\Delta p(1, s) & =\rho \bar{w}_{l}(1, s) \frac{\partial}{\partial x_{l}} \Delta \phi(1, s)
\end{aligned}
$$


where $l$ indicates the direction tangent to the blade surface whose projection on the meridional direction gives the $m$-direction in the meridional plane. Note that $x_{l}$ has the dimension of length. The blade angle has been defined in Eqn. (1.5), from which the following relation is obtained

$$
\sin \beta=\frac{\partial x_{m}}{\partial x_{l}}=\frac{w_{m}}{w_{l}}
$$

where the latter identity follows from the alignment of $\boldsymbol{w}$ with the blade. Substitution of Eqns. (3.1) and (3.9) into Eqns. (3.7) and (3.8) gives

$$
\begin{aligned}
& \Delta p(0, s)=\frac{2 \pi}{Z} \rho \bar{w}_{m}(0, s) \frac{\partial\left(r \bar{v}_{\theta}\right)}{\partial x_{m}}(0, s) \\
& \Delta p(1, s)=\frac{2 \pi}{Z} \rho \bar{w}_{m}(1, s) \frac{\partial\left(r \bar{v}_{\theta}\right)}{\partial x_{m}}(1, s)
\end{aligned}
$$

At the trailing edge the Kutta condition states that $p_{s s}=p_{p s}$ and at the leading edge the zero-incidence condition implies the same. These conditions are always satisfied when

$$
\begin{aligned}
& \frac{\partial\left(r \bar{v}_{\theta}\right)}{\partial m}(0, s)=0 \\
& \frac{\partial\left(r \bar{v}_{\theta}\right)}{\partial m}(1, s)=0
\end{aligned}
$$

This results in two additional constraints for the mean-swirl distribution. Eqn. (3.12) is referred to as the zero-incidence or shock-free condition and Eqn. (3.13) as the Kutta condition for the inverse-design method. Note that for the inverse-design method, the Kutta condition is satisfied via Eqn. (3.13), and the Kutta condition formulated in Eqn. (2.26) for the direct method, does not have to be applied separately.

Summarizing, in order to obtain an impeller with target performance the mean-swirl distribution is described by

$$
r \bar{v}_{\theta}(m, s)=\frac{g H}{\Omega} f(m, s)
$$

The non-dimensional function $f(m, s)$ therefore must satisfy the following constraints

1. no pre-swirl at the leading edge: $f(0, s)=0$

2. desired total pressure increase at the trailing edge: $f(1, s)=1$

3. zero-incidence at the leading edge: $\frac{\partial f}{\partial m}(0, s)=0$

4. Kutta condition at the trailing edge: $\frac{\partial f}{\partial m}(1, s)=0$

In order to prescribe the mean-swirl distribution, a distribution is specified at the hub $f(m, 0)$ and at the shroud $f(m, 1)$. Both these distributions have to satisfy the constraints formulated above. An interpolation function $g(s)$ in span-wise direction is employed to specify the span-wise variation.

$$
f(m, s)=f(m, 0)+g(s)[f(m, 1)-f(m, 0)]
$$


The span-wise interpolation function $g(s)$ has to satisfy $g(0)=0$ and $g(1)=1$. The linear interpolation function, $g(s)=s$, is used unless mentioned otherwise.

Impellers are sometimes designed to have a small incidence at the leading edge, this implies that the zero-incidence condition, given by Eqn. (3.12), is not mandatory. Since the method presented here assumes blades of zero thickness, a zero incidence angle is needed for potential flow considerations, since otherwise a singularity occurs at the leading edge. This is not the case for blades with non-zero thickness.

\subsubsection{Inverse-design algorithm}

By introducing a prescribed mean-swirl distribution, at the blade surface two boundary conditions are specified in the flow problem formulation, the impenetrability condition given in Eqn. (2.24) and the mean-swirl condition formulated in Eqn. (3.1). Only one of these conditions is needed to solve the Laplace equation for a fixed geometry. For the solution of the inverse-design problem both conditions must be satisfied. The following basic iterative algorithm is employed for the inverse-design method:

One boundary condition is used to solve the flow field for a fixed geometry, while the other is used to adjust the blade geometry. This process is repeated until this other boundary condition is also satisfied.

In the iterative approach used here the mean-swirl condition is employed as boundary condition in the solution of the Laplace equation and the impenetrability condition is utilized to adjust the blade shape until both conditions are satisfied.

The algorithm for the inverse-design method is displayed in Fig. 3.2. It starts by specifying the design conditions, followed by the selection of an initial blade shape. The Laplace equation is solved on a Finite Element mesh generated for the current shape, using the mean-swirl distribution as a boundary condition, yielding the velocity potential $\phi$ in the nodal points. In the next step the velocity vector field $\boldsymbol{v}$ is obtained from the computed potential field. The velocity components are then used to adjust the blade shape in order to satisfy the impenetrability condition. If convergence has not been obtained, these steps are repeated until the change in blade shape is sufficiently small to assume convergence. The final result is an inversely-designed blade, which has the prescribed performance in terms of the prescribed mean-swirl distribution, i.e. with the prescribed pump head $H$, zero incidence at the leading edge and the Kutta condition satisfied at the trailing edge.

\subsubsection{Impenetrability condition}

In order to solve the inverse-design problem, the impenetrability condition from Eqn. (2.24) is employed to iteratively change the blade shape. The only unknown for the blade shape is the distribution of $\theta(m, s)$, since $r(m, s)$ and $z(m, s)$ are fixed via the prescribed meridional shape of the blade. Therefore Eqn. (2.24) needs to be rewritten as a partial differential equation for $\theta$. In the numerical implementation of Eqn. (2.24) the computed velocity 


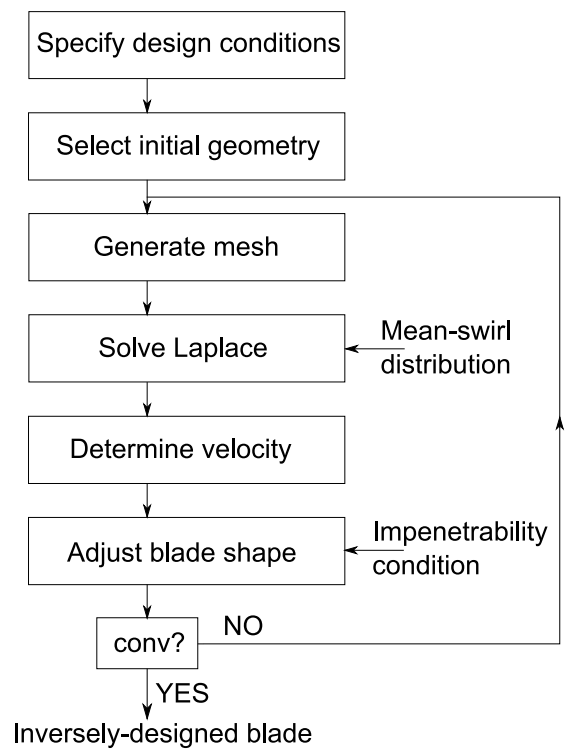

Figure 3.2: Inverse-design algorithm.

components at iteration $n$ are used to obtain the new geometry for iteration $n+1$

$$
\boldsymbol{w}^{(n)} \cdot \boldsymbol{n}^{(n+1)}=0
$$

The surface normal vector $\boldsymbol{n}$ is a function of the blade coordinates $(r, \theta, z)$, where $r, z$ are taken from the prescribed meridional geometry, and $\theta$ is the unknown blade coordinate.

The impenetrability condition is to be formulated in terms of the curvilinear coordinates $m$ and $s$. For this purpose an expression is needed for the surface normal $\boldsymbol{n}$. Therefore the two vectors $\partial \boldsymbol{x} / \partial m$ and $\partial \boldsymbol{x} / \partial s$, tangent to the blade, need to be determined. For the general case the tangent vector $\partial \boldsymbol{x} / \partial t$ along a curve is parameterized by $r=r(t), \theta=\theta(t), z=z(t)$. Using cylindrical coordinates, i.e. $x=r \cos \theta, y=r \sin \theta$, $z=z$ the following expression for $\partial \boldsymbol{x} / \partial t$ is obtained

$$
\begin{aligned}
\frac{\partial \boldsymbol{x}}{\partial t} & =\frac{\partial x}{\partial t} \boldsymbol{e}_{x}+\frac{\partial y}{\partial t} \boldsymbol{e}_{y}+\frac{\partial z}{\partial t} \boldsymbol{e}_{z} \\
& =\left(\frac{\partial r}{\partial t} \cos \theta-r \sin \theta \frac{\partial \theta}{\partial t}\right) \boldsymbol{e}_{x}+\left(\frac{\partial r}{\partial t} \sin \theta+r \cos \theta \frac{\partial \theta}{\partial t}\right) \boldsymbol{e}_{y}+\frac{\partial z}{\partial t} \boldsymbol{e}_{z} \\
& =\frac{\partial r}{\partial t} \boldsymbol{e}_{r}+r \frac{\partial \theta}{\partial t} \boldsymbol{e}_{\theta}+\frac{\partial z}{\partial t} \boldsymbol{e}_{z}
\end{aligned}
$$

where $\boldsymbol{e}_{x}, \boldsymbol{e}_{y}, \boldsymbol{e}_{z}, \boldsymbol{e}_{r}$ and $\boldsymbol{e}_{\theta}$ are the unit vectors in $x, y, z, r$ and $\theta$ directions, respectively. Therefore, if the meridional direction $m$ and $s$ are used to define the tangent vectors, the following expressions are obtained

$$
\begin{gathered}
\frac{\partial \boldsymbol{x}}{\partial m}=\frac{\partial r}{\partial m} \boldsymbol{e}_{r}+r \frac{\partial \theta}{\partial m} \boldsymbol{e}_{\theta}+\frac{\partial z}{\partial m} \boldsymbol{e}_{z} \\
\frac{\partial \boldsymbol{x}}{\partial s}=\frac{\partial r}{\partial s} \boldsymbol{e}_{r}+r \frac{\partial \theta}{\partial s} \boldsymbol{e}_{\theta}+\frac{\partial z}{\partial s} \boldsymbol{e}_{z}
\end{gathered}
$$


The normal vector $\boldsymbol{n}$ can now easily be determined by taking the vector cross product of the directional vectors $\partial \boldsymbol{x} / \partial m$ and $\partial \boldsymbol{x} / \partial s$

$$
\begin{array}{r}
\boldsymbol{n}^{*}=\frac{\partial \boldsymbol{x}}{\partial m} \times \frac{\partial \boldsymbol{x}}{\partial s}=r\left(\frac{\partial \theta}{\partial m} \frac{\partial z}{\partial s}-\frac{\partial \theta}{\partial s} \frac{\partial z}{\partial m}\right) \boldsymbol{e}_{r}+\left(\frac{\partial z}{\partial m} \frac{\partial r}{\partial s}-\frac{\partial z}{\partial s} \frac{\partial r}{\partial m}\right) \boldsymbol{e}_{\theta} \\
+r\left(\frac{\partial \theta}{\partial s} \frac{\partial r}{\partial m}-\frac{\partial \theta}{\partial m} \frac{\partial r}{\partial s}\right) \boldsymbol{e}_{z} \\
\boldsymbol{n}=\frac{\boldsymbol{n}^{*}}{\left\|\boldsymbol{n}^{*}\right\|}
\end{array}
$$

where $\boldsymbol{n}^{*}$ is the unscaled normal vector. Since the right hand side of Eqn. (2.24) equals zero, the impenetrability condition can also be written as follows

$$
\boldsymbol{w} \cdot \boldsymbol{n}=\boldsymbol{w} \cdot \boldsymbol{n}^{*}=0
$$

By substituting Eqn. (3.22) in Eqn. (3.24), using $\boldsymbol{w}=w_{r} \boldsymbol{e}_{\boldsymbol{r}}+w_{\theta} \boldsymbol{e}_{\boldsymbol{\theta}}+w_{z} \boldsymbol{e}_{\boldsymbol{z}}$, the following partial differential differential equation is obtained

$$
\begin{gathered}
\frac{\partial \theta}{\partial m}=a(m, s) \frac{\partial \theta}{\partial s}+b(m, s) \\
a(m, s)=\frac{\left(w_{r} \frac{\partial z}{\partial m}-w_{z} \frac{\partial r}{\partial m}\right)}{\left(w_{r} \frac{\partial z}{\partial s}-w_{z} \frac{\partial r}{\partial s}\right)} \\
b(m, s)=\frac{w_{\theta}}{r} \frac{\left(\frac{\partial r}{\partial m} \frac{\partial z}{\partial s}-\frac{\partial z}{\partial m} \frac{\partial r}{\partial s}\right)}{\left(w_{r} \frac{\partial z}{\partial s}-w_{z} \frac{\partial r}{\partial s}\right)}
\end{gathered}
$$

The meridional blade geometry, given by $r(m, s), z(m, s)$, is prescribed and therefore $\partial r / \partial m, \partial r / \partial s, \partial z / \partial m, \partial z / \partial s$ are known. Note that the relative velocity components $w_{r}$, $w_{\theta}, w_{z}$ are taken from the previous iteration, as is described in Eqn. (3.16).

If the derivatives are taken with respect to the radial direction $m=r$ and the axial direction $s=z$, Eqn. (3.25) can be written as

$$
w_{r} \frac{\partial \theta}{\partial r}+w_{z} \frac{\partial \theta}{\partial z}=\frac{w_{\theta}}{r}
$$

Equation (3.25) is a hyperbolic equation which is to be solved in the meridional plane. The 'initial' condition is given by the prescribed stacking condition $\theta_{t e}(s)$ at the trailing edge, which defines the shape of the trailing edge from hub to shroud

$$
\theta(1, s)=\theta_{t e}(s)
$$

Extra boundary conditions may be needed at $s=0$ (hub) or $s=1$ (shroud). At the hub and at the shroud the velocity is aligned to hub and shroud surfaces, respectively. These surfaces are surfaces of revolution. The meridional tangential vector $\boldsymbol{y}_{\boldsymbol{m}}=$ 


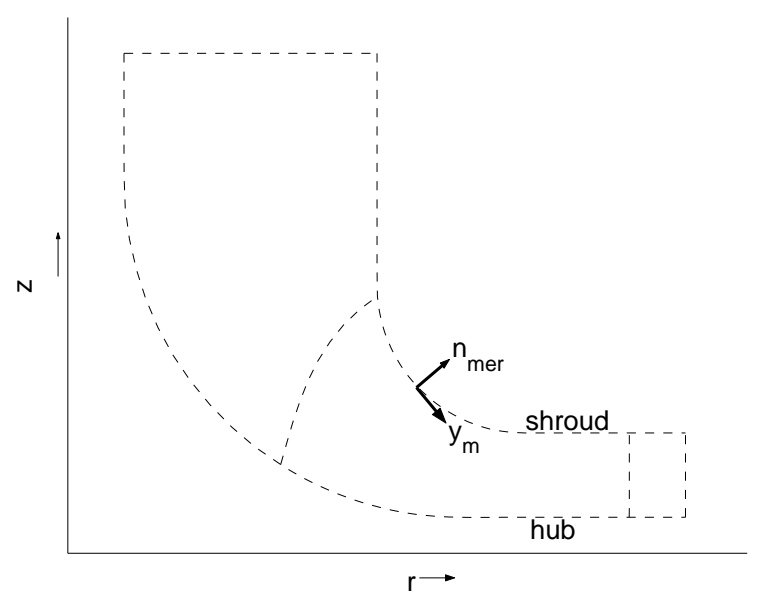

Figure 3.3: Meridional tangential vector and the corresponding meridional normal vector along the shroud

$(\partial r / \partial m) \boldsymbol{e}_{\boldsymbol{r}}+(\partial z / \partial m) \boldsymbol{e}_{\boldsymbol{z}}$ in the meridional plane $(r, z$-plane $)$ and the corresponding normal vector $\boldsymbol{n}_{m e r}=-(\partial z / \partial m) \boldsymbol{e}_{\boldsymbol{r}}+(\partial r / \partial m) \boldsymbol{e}_{\boldsymbol{z}}$ are depicted in Fig. 3.3. The condition that the flow be aligned to the hub and shroud, which are both surfaces of revolution, is

$$
\boldsymbol{w} \cdot \boldsymbol{n}_{m e r}=-w_{r} \frac{\partial z}{\partial m}+w_{z} \frac{\partial r}{\partial m}=0
$$

for $s=0$ (hub) and $s=1$ (shroud). Therefore, at the hub and the shroud, the coefficient $a$ in Eqn. (3.25) equals zero and the partial differential equation reduces to a linear ordinary differential equation for $\theta$, both at hub and at shroud

$$
\frac{d \theta}{d m}=b(m, s)
$$

This means that in order to obtain $\theta$-values at the hub and the shroud, only information is needed along the meridional hub and shroud line, respectively. Thus the hub and shroud contours are characteristics of the hyperbolic equation formulated in Eqn. (3.25) and no extra boundary condition is needed at the hub and shroud. Summarizing, the impenetrability equation is given by Eqn. (3.25), with the initial condition specified by the stacking condition at the trailing edge. By solving this equation an update is obtained for the blade shape.

\subsection{Numerical Implementation}

The numerical implementation of the inverse-design method is discussed in this section. The section gives descriptions of an initial estimate for the blade shape, the flow solution, the blade shape adjustment and furthermore, a comparison of the current method with other inverse-design methods. 


\subsubsection{Quasi 3D initial estimate}

The initial estimate of the blade shape is an important quantity for the iterative approach. The closer the initial estimate is to the blade shape corresponding to the solution of the inverse-design problem, the fewer iterations are usually needed to achieve convergence.

A first possibility is to select a blade with a constant blade angle. The unknown coordinates $\theta$ for the initial blade shape can then be computed from the definition of the blade angle $\beta$ (see Eqn. (1.6)).

$$
\tan \beta=\frac{1}{r} \frac{d x_{m}}{d \theta}=\frac{w_{m}}{w_{\theta}}
$$

Note that the latter part of this equation is not required if a constant blade angle is prescribed.

For a quasi two-dimensional geometry a good estimate can be found for the blade angle at the leading edge from velocity triangles using $v_{\theta, l e}=0$ and for the blade angle at the trailing edge $\beta_{t e}$ from the Euler pump equation (for both see section 1.2). Therefore, a linear interpolation of the blade angle between the leading and trailing edge can also be utilized to obtain an initial estimate for the blade shape.

Another approach is to use a quasi three-dimensional approach (Q3D), i.e. the velocity in the flow channel is assumed to be uniform from blade to blade. This corresponds to the case of an infinite number of blades.

For such an impeller the velocity $w_{\theta}$ is constant from blade to blade and can be obtained from the prescribed mean-swirl distribution prescribed by Eqn. (3.14), i.e.

$$
w_{\theta}=v_{\theta}-\Omega r \cong \frac{r \bar{v}_{\theta}}{r}-\Omega r
$$

For a quasi two-dimensional geometry, with no variation in the $z$-direction, the meridional velocity $w_{m}$ can be estimated from

$$
w_{m}=w_{r}=\frac{Q}{2 \pi r b}
$$

where $b$ is the width of the impeller. For a fully three-dimensional geometry, the meridional velocity $w_{m}$ can be obtained from a single flow computation without the presence of blades, or in other words for a first iteration with $\phi^{+}-\phi^{-}=0$ at the blade surface. The initial estimate is found by rewriting Eqn. (3.32) as

$$
\frac{d \theta}{d x_{m}}=\frac{w_{\theta}}{r w_{m}}
$$

where $x_{m}$ is the (unscaled) meridional length.

\subsubsection{Flow solution}

The solution of the Laplace equation for the velocity potential $\phi$ is obtained, using the Finite Element Method as described in section 2.4. At the blade surface the periodic boundary condition, as specified in Eqn. (3.1), is employed. This means that, unless convergence is obtained, the flow solution will not satisfy the impenetrability condition from Eqn. (2.24), which is employed to adjust the blade shape instead. 


\subsubsection{Blade shape adjustment}

In section 3.2.5 it has been discussed how the blade shape is changed iteratively by solving a partial differential equation that corresponds to the impenetrability condition, $\boldsymbol{w} \cdot \boldsymbol{n}=0$. Here the numerical implementation of the adjustment procedure is elucidated. Firstly, the discretization of the impenetrability equation is treated. Secondly, under-relaxation of the update equation is described. The final section is devoted to the adopted convergence criteria.

\section{Discretization of the impenetrability condition}

During each iteration a new blade shape is found utilizing the impenetrability condition, given in Eqn. (3.25). The numerical approach is summarized by

- the initial condition is the stacking condition at the trailing edge, Eqn. (3.29).

- the ordinary differential equation (3.31) is solved for the hub and the shroud curve.

- the partial differential equation (3.25) is solved for the internal blade domain in the meridional plane.

The ordinary differential equation (3.31) is solved at the hub and the shroud by using a second order Runge-Kutta scheme. For the internal domain the partial differential equation (3.25) is solved by employing the implicit Crank-Nicolson scheme, which is of second order accuracy in $\Delta m$ and $\Delta s$. The scheme is given by

$$
\frac{\theta_{i+1, j}-\theta_{i, j}}{\Delta m}=\frac{1}{2} \frac{a_{i+1, j}\left(\theta_{i+1, j+1}-\theta_{i+1, j-1}\right)+a_{i, j}\left(\theta_{i, j+1}-\theta_{i, j-1}\right)}{2 \Delta s}+\frac{b_{i+1, j}+b_{i, j}}{2}
$$

where $i$ is the index in the $m$-direction and $j$ the index in the direction of $s$-direction. The solutions for the hub and the shroud are employed here as well. The equation is solved from trailing to leading edge (in the $m$-direction). Note that the equations are coupled in span-wise direction $s$.

\section{Under-relaxation}

Using the approach outlined in the preceding sections, an update $\theta^{u}(m, s)$ is obtained. However, in order to achieve convergence of the iterative problem, it is often necessary to use under-relaxation

$$
\theta^{n+1}=\theta^{n}+\omega \Delta \theta
$$

where $\omega$ is the relaxation coefficient, $n$ the iteration number and $\Delta \theta=\theta^{u}-\theta^{n}$. Usually the finer the mesh, the smaller $\omega$ will need to be in order to obtain convergence. An additional approach to increase the robustness of the method is to use a maximum change in $\theta$ per iteration, $\Delta \theta_{\text {lim }}$

$$
\begin{aligned}
& \Delta \theta=\theta^{u}-\theta^{n} \\
& \text { if }|\Delta \theta|<\Delta \theta_{\text {lim }} \\
& \Delta \theta=\Delta \theta_{\text {lim }} \\
& \text { if } \quad \Delta \theta>\Delta \theta_{\text {lim }} \\
& \Delta \theta=-\Delta \theta_{\text {lim }} \\
& \text { if } \Delta \theta<-\Delta \theta_{\text {lim }}
\end{aligned}
$$




\section{Convergence}

During the iteration process the blade shape changes. Hence, a convergence criterion is needed to determine whether convergence has been obtained. The adopted criterion is that $\Delta \theta_{\text {max }}$, the maximum of the change in $\theta$ over all nodes $i, j$ on the blade before relaxation, is smaller than a specified tolerance $\epsilon$, i.e.

$$
\Delta \theta_{\max }=\max _{i, j}\left|\theta_{i, j}^{n+1}-\theta_{i, j}^{n}\right|<\epsilon
$$

Alternatively, deviations from the impenetrability condition (2.24) can be considered. It is preferred to use a dimensionless criterion for this purpose. In the numerical discretization, the blade surface consists of surface triangles. The net flow through the blade surface $Q_{b l}$ is computed by calculating the flow through each surface triangle $p$. This residual quantity $R_{Q}$ is made dimensionless by dividing by $Q$, the flow through the pump, which is one of the design parameters.

$$
R_{Q}=\frac{\left|Q_{b l}\right|}{Q}=\frac{\sum_{p=1}^{m}\left|w_{n}^{p}\right| A_{t}^{p}}{Q}
$$

where $m$ is the number of triangles on the blade surface, $A_{t}^{p}$ the surface area of triangle $p$ and $\left|w_{n}^{p}\right|$ the absolute value of the normal component of the relative velocity. For an infinitely fine mesh, $R_{Q}=0$.

\subsubsection{Comparison with other methods}

The proposed inverse-design method is based on similar principles as the method presented by Borges [6] and Zangeneh [80]. The difference in approach is mainly in the numerical approach of the problem. In the present method the Laplace equation is solved using the Finite Element Method, whereas in the method of Borges [6] the Laplace equation is expanded into a Fourier series in the circumferential direction $\theta$. The amplitudes of these Fourier modes, which depend on $r, z$, satisfy a Helmholtz-type equation. The number of these Helmholt-type equations depends on the order of the Fourier series. Each of these equations is then solved numerically with appropriate boundary conditions. Furthermore, in the method by Borges the update equation is solved along meridional lines, in a decoupled fashion (along the span). In the method proposed here, the update method is coupled in span-wise direction from hub to shroud. In the experience of the author, in the method of Borges a relative low resolution is frequently used from blade to blade to avoid convergence problems, whereas in the current method generally a larger number of elements from blade to blade can be used, while still obtaining a converged solution. Finally, the previous method is known to have problems for impellers with few blades. The method proposed here works well for such cases, although for a low number of blades generally more under-relaxation is needed in the update procedure. 


\subsection{Verification cases}

In this section two verification cases are presented for the developed inverse-design method. Both are quasi two-dimensional cases, i.e. they are two-dimensional cases solved on a three-dimensional mesh. The first verification case deals with a source with vortex flow. This case has no direct connection to turbomachines, but it is a good verification case, since the analytical solution for the inverse-design problem can be derived. The second case deals with a quasi two-dimensional radial pump geometry.

\subsubsection{D source with vortex}

By combining two elementary potential flows a verification case can be obtained. These elementary flows are a source flow and a free-vortex flow. For this two-dimensional case the analytical solution for the potential $\phi(r, \theta)$ is known

$$
\phi(r, \theta)=\frac{q}{2 \pi} \ln r+\frac{\gamma}{2 \pi} \theta
$$

where $q$ is the two-dimensional flow rate, i.e. the flow rate per unit length with the third dimension given by the width of the impeller $b$

$$
q=\frac{Q}{b}
$$

and $\gamma$ is the constant circulation given by

$$
\gamma=\phi^{+}-\phi^{-}
$$

The relative velocity components $w_{r}$ and $w_{\theta}$ are determined from Eqn. (3.43)

$$
\begin{gathered}
w_{r}=v_{r}=\frac{\partial \phi}{\partial r}=\frac{q}{2 \pi r} \\
w_{\theta}=v_{\theta}-\Omega r=\frac{1}{r} \frac{\partial \phi}{\partial \theta}-\Omega r=\frac{\gamma}{2 \pi r}-\Omega r
\end{gathered}
$$

Note that both components depend on $r$ only. The velocity potential and hence the velocity itself are known everywhere. They are not influenced by the position of the 'blade' in this case. Thus the mean-swirl distribution and the potential jump are independent of the meridional distance $m$, see Eqn. (3.45).

Subsequently, the 'blade' shape is determined, such that it is aligned with the direction of the relative flow. In the quasi two-dimensional case the impenetrability condition, Eqn. (3.28), is

$$
w_{r} \frac{d \theta}{d r}=\frac{w_{\theta}}{r}
$$

Substituting the relative velocity components from Eqns. (3.46) and (3.47) in (3.48) the following ordinary differential equation for $\theta$, the angular coordinate of the 'blade', is obtained

$$
\frac{d \theta}{d r}=\frac{w_{\theta}}{w_{r} r}=\frac{\gamma}{q r}-\frac{2 \pi \Omega r}{q}
$$



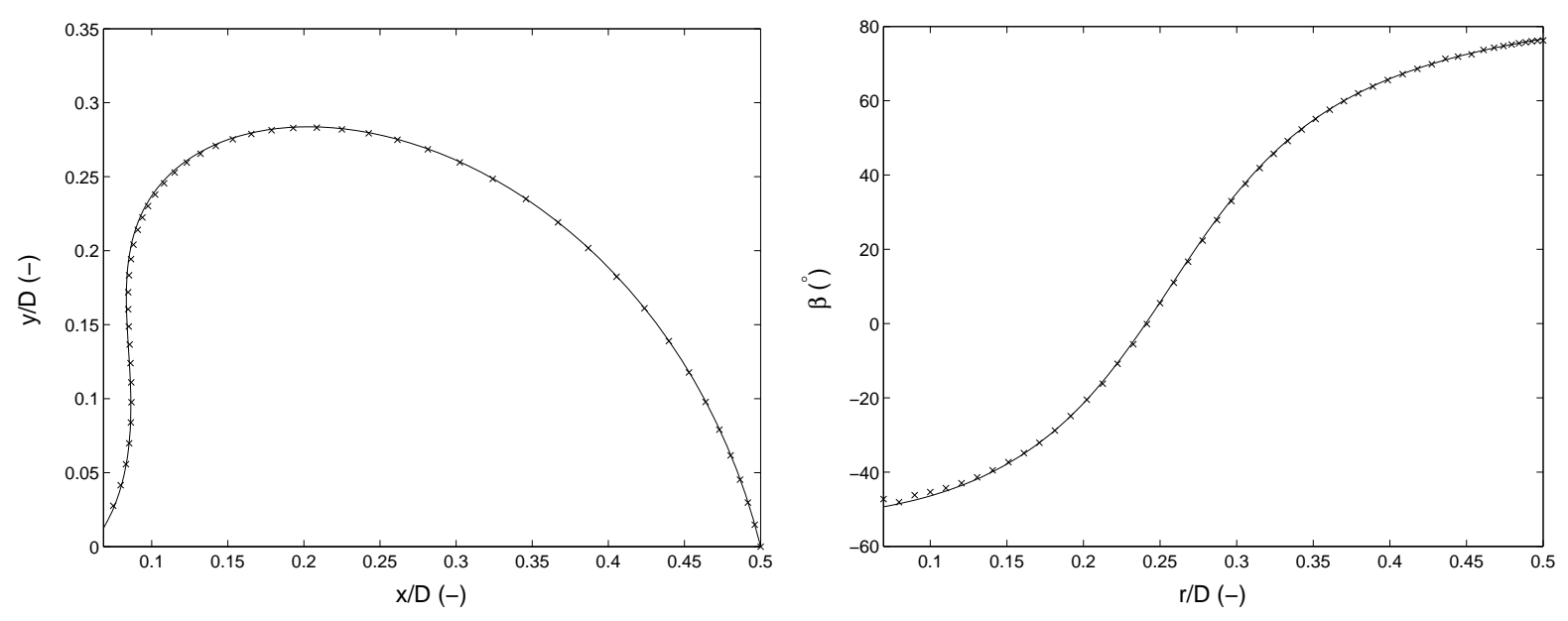

Figure 3.4: 'Blade' shape (left) and blade angles (right) for the source with vortex verification case. The analytical solution is given by the lines and the crosses mark the numerical solution. $\gamma=7.62 \mathrm{~m}^{2} / \mathrm{s}, q=6.0 \mathrm{~m}^{2} / \mathrm{s}, \Omega=40.4 \mathrm{rad} / \mathrm{s}, r_{t e}=0.36 \mathrm{~m}$ and $\theta_{t e}=0.0 \mathrm{rad}$. The mesh parameters are $n i=50, n j=15$ and $n k=2$.

The integration of this equation is straightforward. The result, when starting at the trailing edge with prescribed polar coordinates $\left(r_{t e}, \theta_{t e}\right)$, is

$$
\theta=\theta_{t e}+\frac{\gamma}{q} \ln \frac{r}{r_{t e}}-\frac{\pi \Omega}{q}\left(r^{2}-r_{t e}^{2}\right)
$$

The analytical solution for the blade angle $\beta$, as defined in Eqn. (1.6), can be determined as well

$$
\tan \beta=\frac{d r}{r d \theta}=\frac{w_{r}}{w_{\theta}}=\frac{q}{\gamma-2 \pi \Omega r^{2}}
$$

To verify the developed method an inverse-design is performed with the periodic boundary condition given by Eqn. (3.45). The numerical result is then compared to the analytical solution given in Eqns. (3.50) and (3.51). The computation has been performed using a three-dimensional mesh with $n i=50, n j=15$ and $n k=2$. Here $n i$ is the number of nodes from inlet to outlet, $n j$ the number of nodes from blade to blade and $n k$ the number of nodes from hub to shroud, for which only 2 nodes are taken since it is a quasi two-dimensional case. The inversely-designed 'blade' agrees very well with the analytically determined shape, as is shown in Fig. 3.4. This verifies the developed method.

\subsubsection{Reproducing a logarithmic blade}

In this second verification case a radial impeller with 7 logarithmic blades is considered. A logarithmic blade is a blade with a constant blade angle $\beta$ from leading to trailing edge. In the case considered the constant blade angle is chosen equal to $20^{\circ}$. The impeller diameter is $0.64 \mathrm{~m}$. The dimensionless performance coefficients (see section 1.1.5) for this impeller are listed in table 3.1. The logarithmic blade will be reproduced by using the mean-swirl 
Table 3.1: Performance characteristics of the logarithmic impeller.

\begin{tabular}{|c|c|c|}
\hline$\phi$ & $\psi$ & $N_{s}$ \\
\hline 0.0073 & 0.13 & 0.40 \\
\hline
\end{tabular}

distribution from a direct analysis of this impeller as input for the inverse-design method. The result of the inverse-design method should be identical to the original logarithmic blade. The procedure is summarized by

- perform a direct computation for a fixed geometry with a direct method.

- determine the potential difference between pressure and suction side (and thus the mean-swirl distribution) from the results of the direct computation.

- carry out an inverse-design computation using the mean-swirl distribution, determined from the direct computation, as input.

- compare the blade shape and the blade angles from the inverse-design method to those of the original geometry.

The same meridional meshes are used for the direct method and for the inverse-design method in order to make the computations more similar. Therefore, the only differences that can occur are in the discretization of the direct and inverse solution steps. The meshes which were used in this approach are summarized in table 3.2. Here $n i$ is the number of nodes in stream-wise direction, $n j$ the number of nodes from blade to blade and $n k$ the number of nodes in span-wise direction, i.e. from hub to shroud. Since the case considered is a quasi two-dimensional case, $n k$ is set to 2. $n_{\text {tets }}$ indicates the number of tetrahedrons in the mesh.

The resulting blade shapes and blade angles are plotted in Fig. 3.5. The blade curves are almost overlapping and can hardly be distinguished from one another. The blade angles are always close to $20^{\circ}$, only near the leading edge for the first few nodes some deviations can be observed. Similar behavior is encountered at the trailing edge. For finer meshes the blade angles are closer to $20^{\circ}$, but the deviations at the leading edge remain. This is caused by the small flow incidence at the leading edge for the original blade, resulting in a weak singularity at the leading edge. This analysis clearly shows the consistency of the results of the direct and the inverse-design method.

Table 3.2: Mesh parameters for the direct and inverse-design computations.

\begin{tabular}{|c|c|c|c|c|}
\hline & $n i$ & $n j$ & $n k$ & $n_{\text {tets }}$ \\
\hline mesh a & 73 & 29 & 2 & 12,096 \\
\hline mesh b & 145 & 57 & 2 & 48,384 \\
\hline mesh c & 289 & 113 & 2 & 193,536 \\
\hline
\end{tabular}



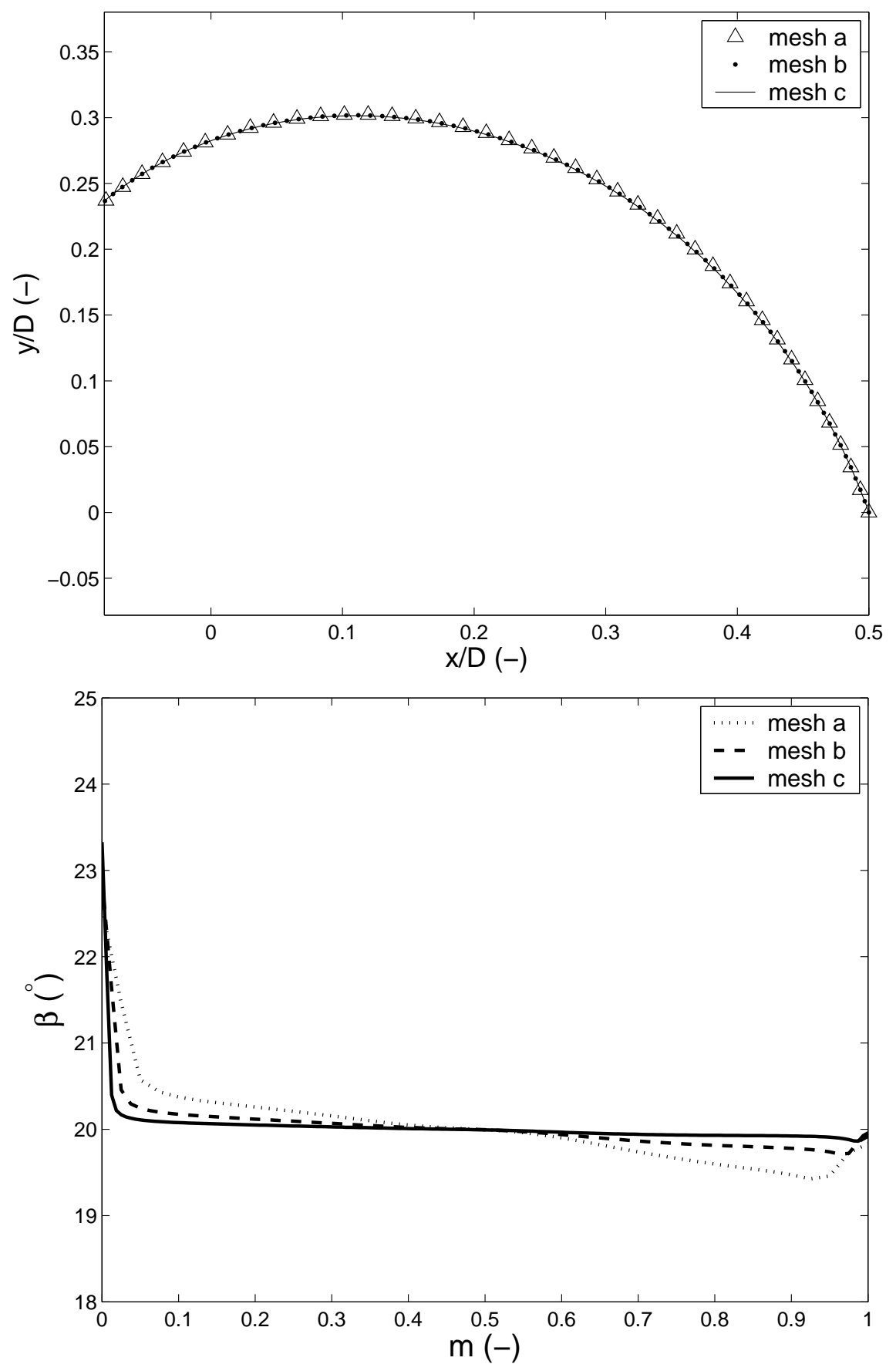

Figure 3.5: Top view of the blade shape (top) and the corresponding blade angles (bottom) for the logarithmic verification case for meshes $a, b$ and $c$. 


\subsubsection{Order of accuracy}

The logarithmic blade case has been used to determine the order of accuracy of the inversedesign method. The $\theta$ at the leading edge at mid-span is computed for three different meshes, that are listed in table 3.3. Richardson extrapolation based on $\theta_{l e}$ can then be used to determine the order of accuracy of the inverse-design method

$$
\theta_{l e}(h)=\theta_{l e, e x a c t}+C h^{p}+o\left(h^{p}\right)
$$

where $C$ and $p$ are unknown constants and $h$ indicates the length scale of the mesh elements. The higher order terms $o\left(h^{p}\right)$ are omitted from consideration.

Table 3.3: Mesh influence for the inverse-design method applied to the logarithmic blade.

\begin{tabular}{|c|c|c|c|c|}
\hline$n i$ & $n j$ & $n k$ & $h / h_{0}$ & $\theta_{l e}(\mathrm{rad})$ \\
\hline 31 & 9 & 3 & 2.0 & 1.8054 \\
\hline 61 & 17 & 5 & 1.0 & 1.8417 \\
\hline 121 & 33 & 9 & 0.5 & 1.8591 \\
\hline$\infty$ & $\infty$ & $\infty$ & 0 & 1.8751 \\
\hline
\end{tabular}

Using the data from table 3.3 the order of the method is determined

$$
\frac{\theta_{l e}(2 h)-\theta_{l e}(h)}{\theta_{l e}(h)-\theta_{l e}(h / 2)}=2^{p} \Rightarrow p=1.1
$$

For the logarithmic blade case the developed inverse-design method appears to be first order accurate. A second-order accurate method is desirable. The potentials from the solution of the Laplace equation and the update discretization are of second order. The determination of the velocities with the SPR-method (see section 2.4.4) is less accurate at the blade surface (a boundary of the solution domain). The relative velocity components at the blade surface are used to update the blade geometry using Eqn. (3.25). The reduced accuracy in the determination of the blade velocity components apparently reduces the method as a whole to first order. If the order of the inverse-design method is to be increased, the order of the scheme to determine the velocity components near boundaries has to be increased, but this has not been carried out in the work presented here. For future work it is recommended to investigate a more accurate method to determine the velocity, especially near domain boundaries of the solution domain. 


\subsection{Inverse-design of radial impeller blades}

The first design case that is considered is the inverse-design of a three-dimensional radial pump impeller. For this purpose the so-called SHF impeller is studied. This impeller was originally designed by the Société Hydrotechnique de France in order to study inlet and outlet recirculation in centrifugal pumps at off-design conditions. It is still used as a reference case for experimental and numerical research (for example in Wuibaut et al. $[77,78])$.

It is a radial impeller with an axial inlet, making it a fully three-dimensional geometry, rather than the quasi $2 \mathrm{D}$ geometries that have been used as verification cases in the preceding section.

In this section first some details about the original design are given, followed by two inverse-design computations. In the first design case a default loading is applied and in the second design case the loading is changed in order to improve the loading at the blades. Finally, the results of the two designs are compared to those of the original design in section 3.5.4.

\subsubsection{SHF impeller original design}

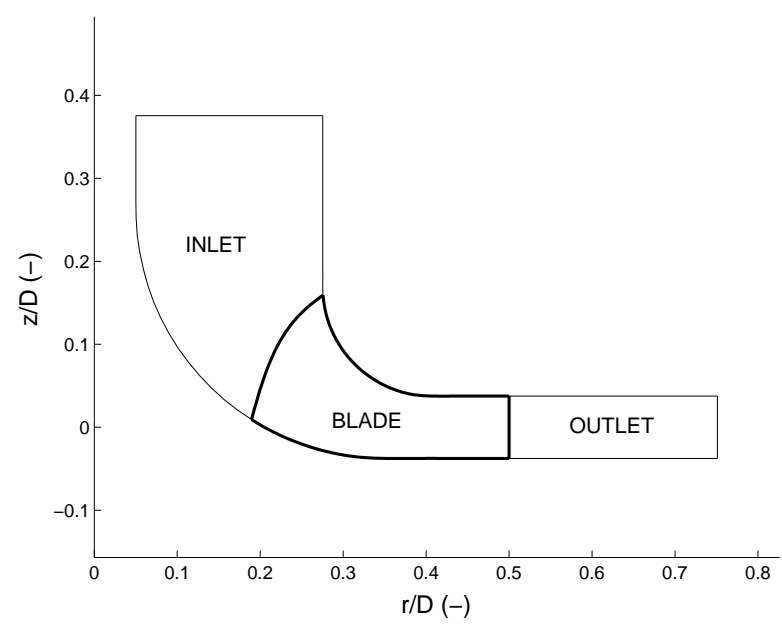

Figure 3.6: Meridional shape of the SHF impeller.

The geometry of the original SHF impeller is discussed here. The meridional shape of the SHF impeller is depicted in Fig. 3.6 including the inlet, blade and outlet sections. The original impeller, which has zero stacking at the trailing edge, and the original blade angle distribution are shown in Fig. 3.7. The blade angles (with respect to the circumferential direction) are between $17^{\circ}$ and $27^{\circ}$. For a large part of the blade, close to the trailing edge, the blade angle is fairly constant with a value around $22^{\circ}$. The design parameters for the impeller are listed in table 3.4. The specific speed $N_{s}$ has been computed from Eqn. (1.9) using the inviscid flow pump head. This ideal pump head has been determined by performing a direct computation for the original geometry with blades without thickness. When investigating the performance of pumps the focus is on cavitation properties, 

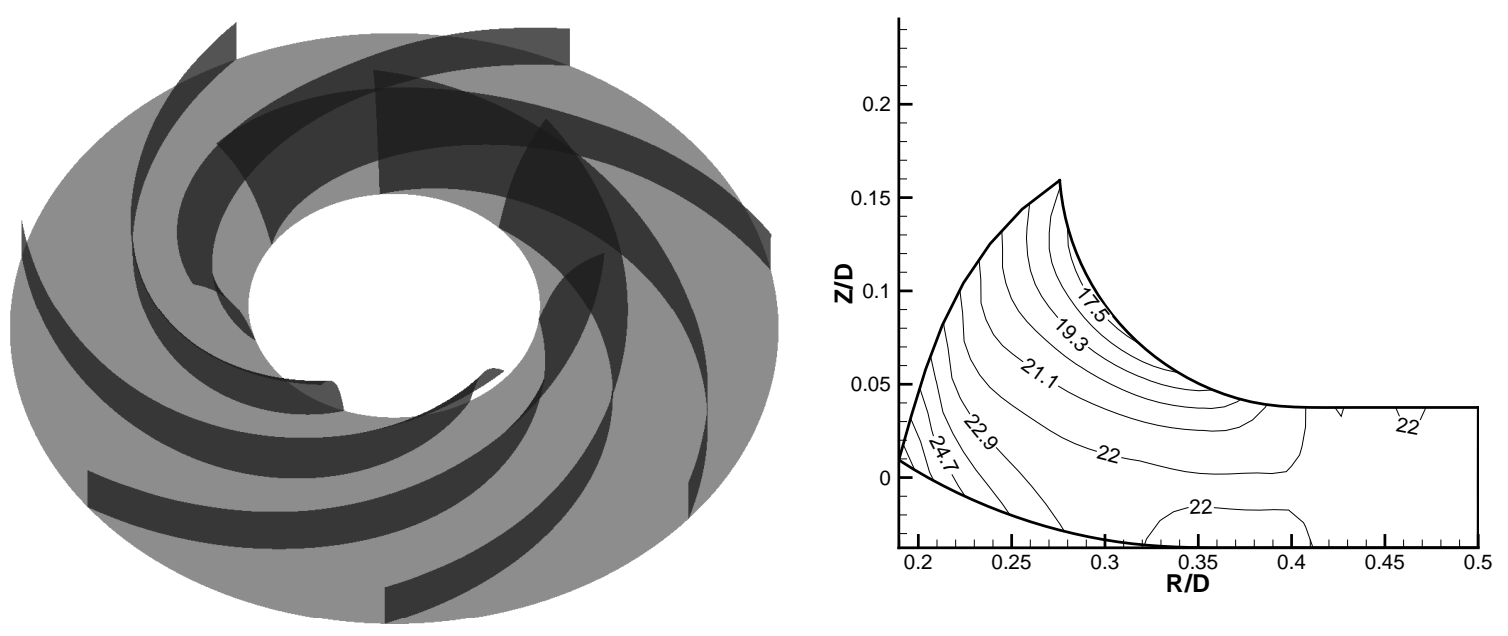

Figure 3.7: Original design of SHF impeller: the impeller (left) and its blade angle distribution $\beta(m, s)$ in the meridional plane (right).

Table 3.4: Dimensionless characteristics of the SHF impeller at design point.

\begin{tabular}{|c|c|c|c|}
\hline$Z$ & $\phi$ & $\psi$ & $N_{s}$ \\
\hline 7 & 0.035 & 0.23 & 0.57 \\
\hline
\end{tabular}
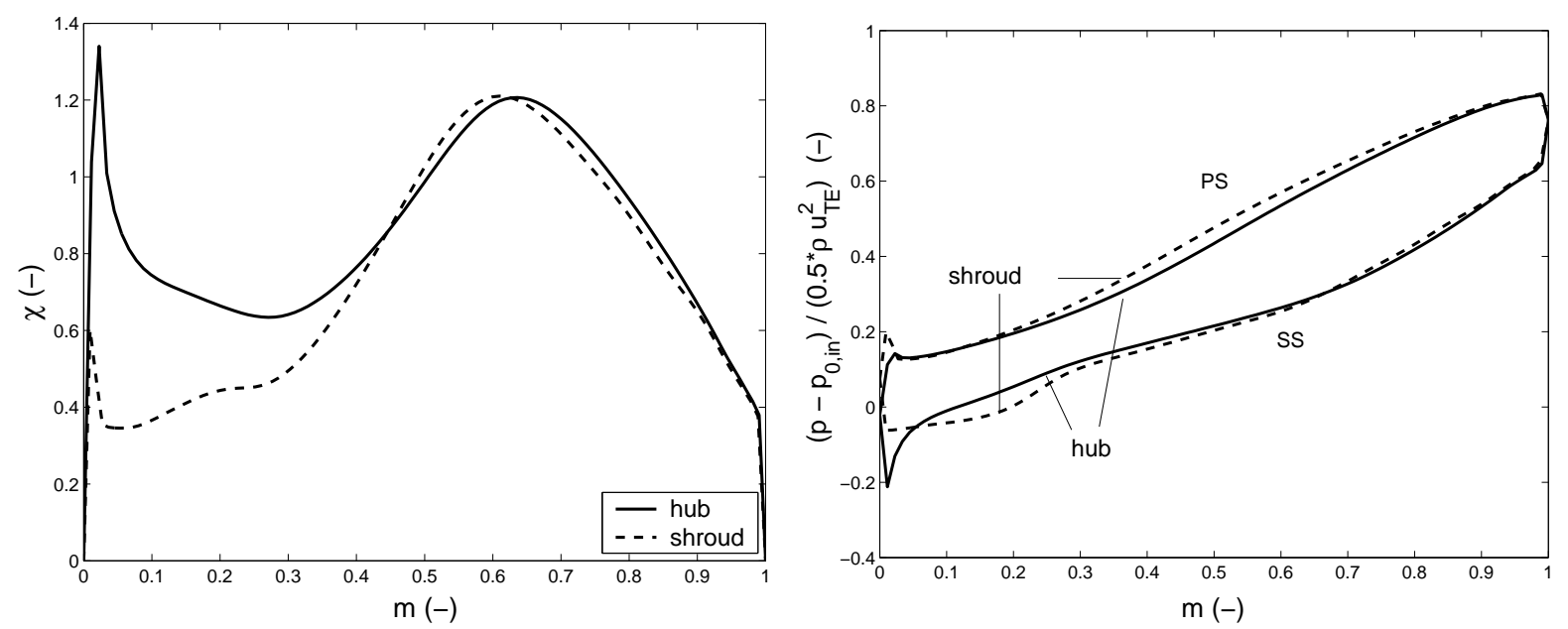

Figure 3.8: Original design of SHF impeller: the velocity loading on the blades (left) and the non-dimensional pressure distribution (right). PS indicates the pressure side and SS the suction side.

boundary-layer losses and velocity loading on the blades. The cavitation performance is expressed by the cavitation inception coefficient $\kappa_{i}$ (see Eqn. (1.10)), the boundary-layer losses are expressed by the loss coefficient $\zeta$ (see Eqn. (2.28)) and the velocity loading $\chi$ on the blades is given by the ratio between the velocity difference between pressure and 
suction side and the average blade velocity

$$
\chi=\frac{w^{s s}-w^{p s}}{\bar{w}}
$$

where $\bar{w}$ is the average of the velocity at the blade pressure and suction side surface, $\bar{w}=1 / 2\left(w^{s s}+w^{p s}\right)$. A general rule in pump design is that this velocity loading should not exceed a certain specified value, frequently the value of 1.0 is employed for this quantity, i.e. $w^{s s}=3 w^{p s}$. The exact value of this (maximum) design target depends on the type of pump under consideration and the maximum values are ranging from 0.7 to 1.5, when going from high to low specific speeds $N_{s}$ [62].

For the cavitation inception coefficient we find $\kappa_{i}=2.65 \cdot 10^{-2}$ and for the loss coefficient $\zeta=2.50 \cdot 10^{-2}$. The velocity loading on the blades is plotted in Fig. 3.8, together with the pressure distribution along the intersection of the blade with the hub and with the shroud. The large gradients observed in both velocity loading and pressure distribution near the leading edge indicate that there is a non-zero incidence at the leading edge for the original geometry. The maximum velocity loading on the blades is $\chi_{\max }=1.34$, which is larger than the general design target of 1.0 referred to above. 


\subsubsection{SHF impeller inverse-design case 1}

For the inverse-design a mean-swirl distribution needs to be specified. The first meanswirl distribution that is selected can be regarded as the simplest polynomial that satisfies the constraints specified in Eqn. (3.14). It is

$$
f(m, s)=3 m^{2}-2 m^{3}
$$

This distribution is constant from hub $(s=0)$ to shroud $(s=1)$ and it is depicted in Fig. 3.9, where it is compared to the mean-swirl distribution for the original geometry along the hub and the shroud, which has been obtained from a direct flow analysis. The parameters specified in table 3.4 are used as input conditions for the inverse-design method.
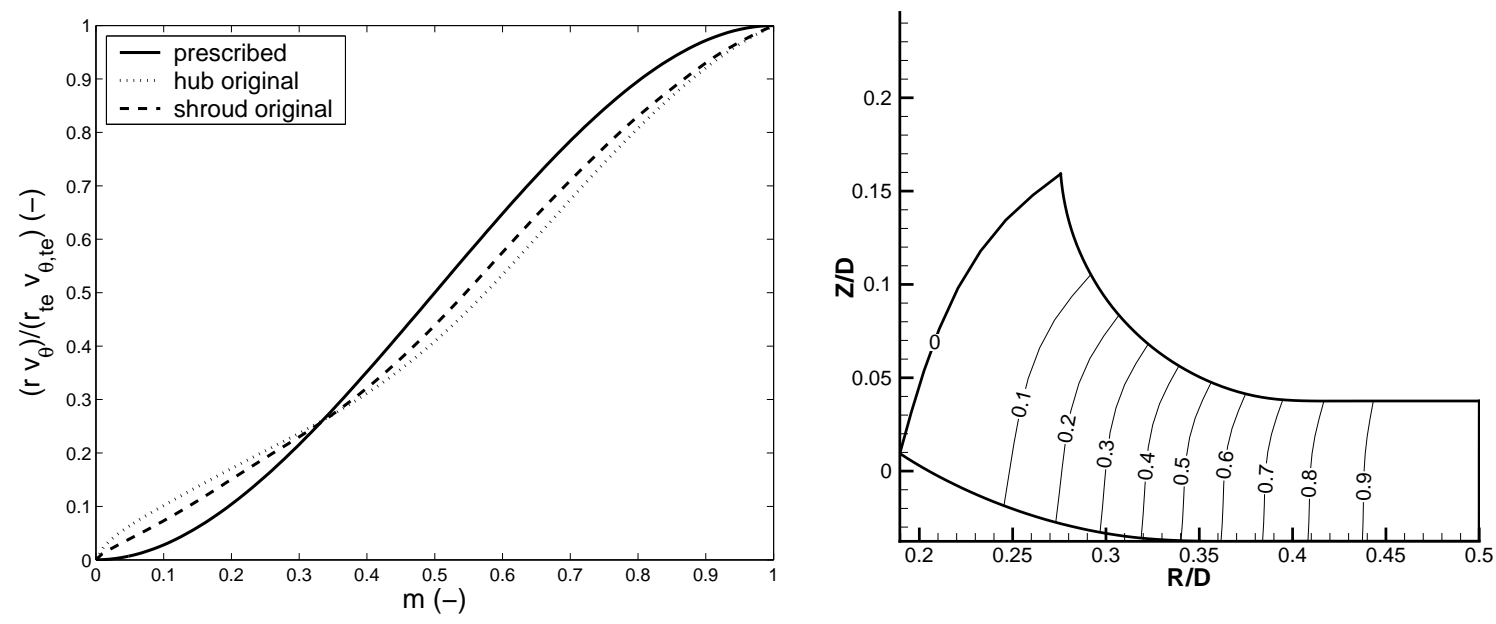

Figure 3.9: SHF impeller inverse-design case 1: the prescribed mean-swirl distribution (constant from hub to shroud) as a function of the meridional length compared to distribution for the original geometry at hub and shroud (left). Contour plot for the prescribed swirl in the meridional plane (right)

Since this is the first fully three-dimensional case that is considered, the influence of the numerical parameters on the results is investigated. For this purpose, the inverse computation has been carried out on three different meshes. The numerical parameters for the inverse computations are listed in table 3.5. Here $n_{\text {tets }}$ is the number of tetrahedrons in the mesh. For the inverse computations a straight blade is taken as initial estimate, in

Table 3.5: Numerical parameters for the SHF impeller.

\begin{tabular}{|c||c|c|c|c||c|c|c|}
\hline mesh & ni & nj & nk & $n_{\text {tets }}$ & $\omega$ & $\Delta \theta_{\text {lim }}$ (rad $)$ & $\epsilon$ \\
\hline \hline coarse & 73 & 15 & 8 & 42,336 & 0.25 & 0.25 & $1.10^{-3}$ \\
\hline medium & 109 & 22 & 12 & 149,688 & 0.25 & 0.25 & $1.10^{-3}$ \\
\hline fine & 145 & 29 & 16 & 362,880 & 0.25 & 0.25 & $1.10^{-3}$ \\
\hline
\end{tabular}

order to check the robustness of the method. 


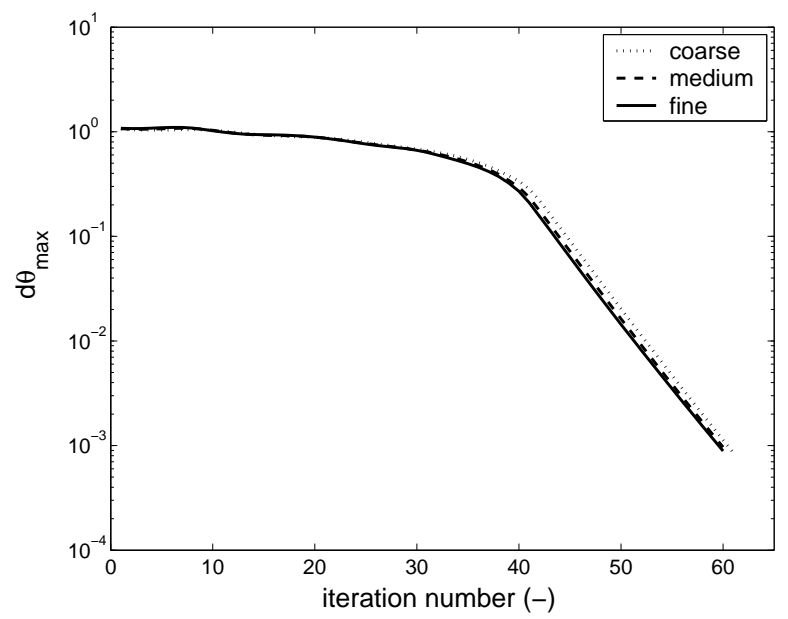

(a) $d \theta_{\max }$

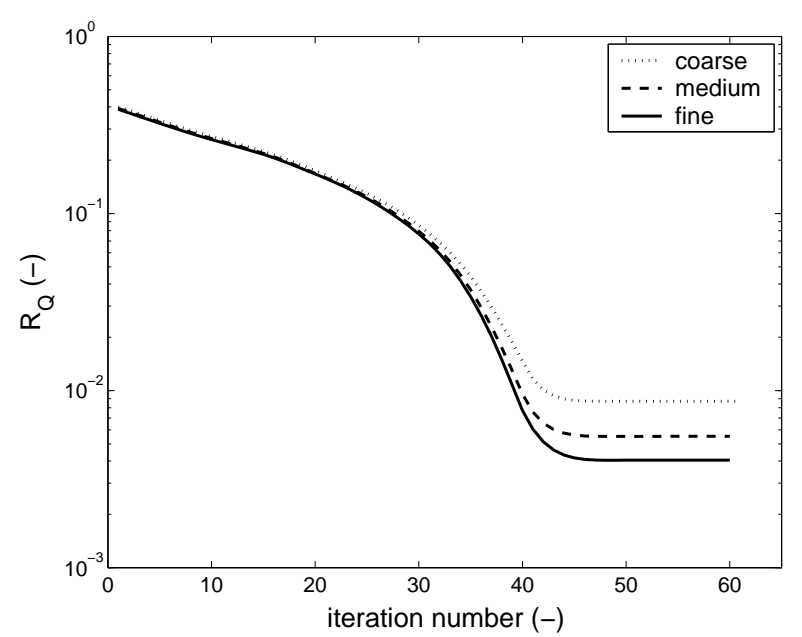

(b) $R_{Q}$

Figure 3.10: SHF impeller inverse-design case 1: the convergence of $d \theta_{\max }$ and $R_{Q}$ shown for the inverse computations on the coarse, medium and fine mesh.

The convergence plots are shown in Fig. 3.10. Note that the iteration process is stopped when $\Delta \theta_{\max }<\epsilon$. The final value of $R_{Q}$ (see Eqn. (3.42)) depends on the mesh size, i.e. it decreases with increasing mesh resolution. Moreover, it is observed that the $\Delta \theta_{\max }$ first decreases slowly and then after about 40 iterations drops rapidly. This is caused by the $\Delta \theta_{\text {lim }}$-criterion, which prevents large changes in the $\theta$-distribution. This criterion increases the robustness of the method greatly, especially when there is a large difference between the initial and final blade shape. The number of iterations needed to achieve convergence is similar for the three considered meshes: 60 for the coarse mesh, 61 for the medium mesh and 60 for the fine mesh.

In Fig. 3.11 the $\theta$-distribution at the hub is given for the resulting inversely-designed blades, in order to investigate the influence of the mesh size on the obtained results. It is clear that for finer meshes the result is mesh independent. From this point onwards the results for the finer mesh are discussed. The iterative process for the fine mesh is illustrated in Fig. 3.12. The largest changes in blade shape occur during the first 10 iterations, after that the blade shape changes slowly.

The resulting inversely-designed impeller and blade angle distribution $\beta(m, s)$ are depicted in Fig. 3.13. The blade angles are between $15^{\circ}$ and $30^{\circ}$ and the blade angles show a much larger variation from leading to trailing edge than for the original design (see also Fig. 3.7). Near the leading edge the differences in blade curvature are most pronounced. The inversely designed blade has a higher degree of twist and is slightly longer.

The velocity loading for this inverse-design case 1 is shown in Fig. 3.14. The velocity loading shows a large variation from leading to trailing edge with a clear maximum. The maximum velocity loading coefficient is $\chi_{\max }=1.74$, which is considerably higher than that found for the original design. The pressure distribution is shown on the right in Fig. 3.14, and the distribution is clearly much smoother than that for the original design, caused by the enforced zero-incidence flow at the leading edge. The cavitation inception coefficient is therefore much lower $\kappa_{i}=0.47 \cdot 10^{-2}$, compared to the original 


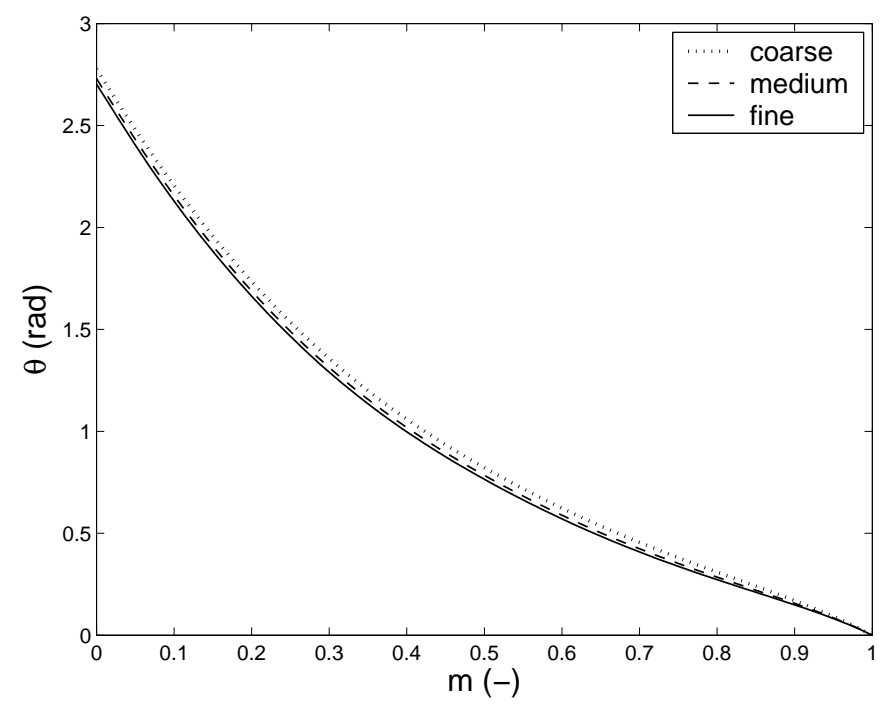

Figure 3.11: SHF impeller inverse-design case 1: the $\theta$-distribution at the hub for a coarse, medium and fine mesh.

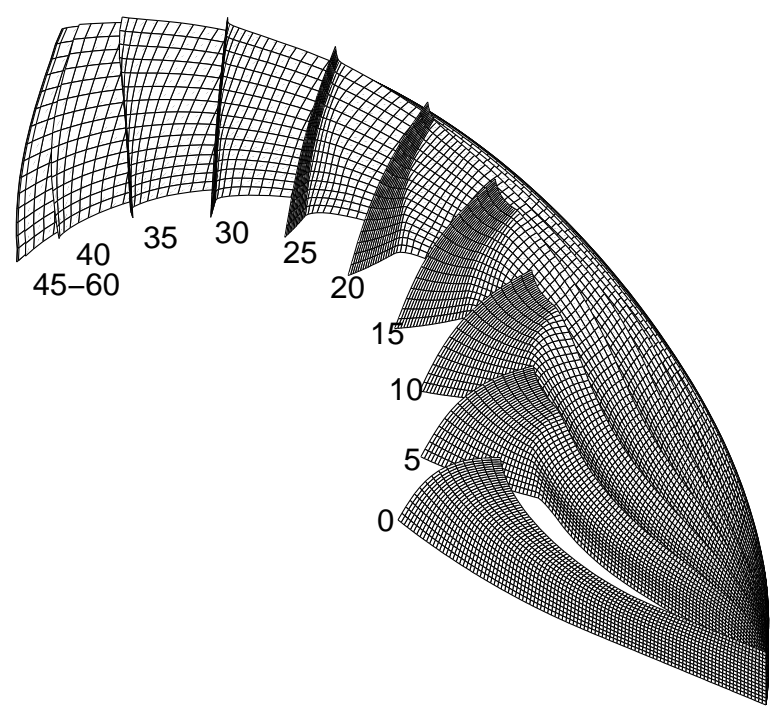

Figure 3.12: SHF impeller inverse-design case 1: iterative blade adjustment. The blade shape is shown after $0,5,10, \ldots, 60$ iterations. 

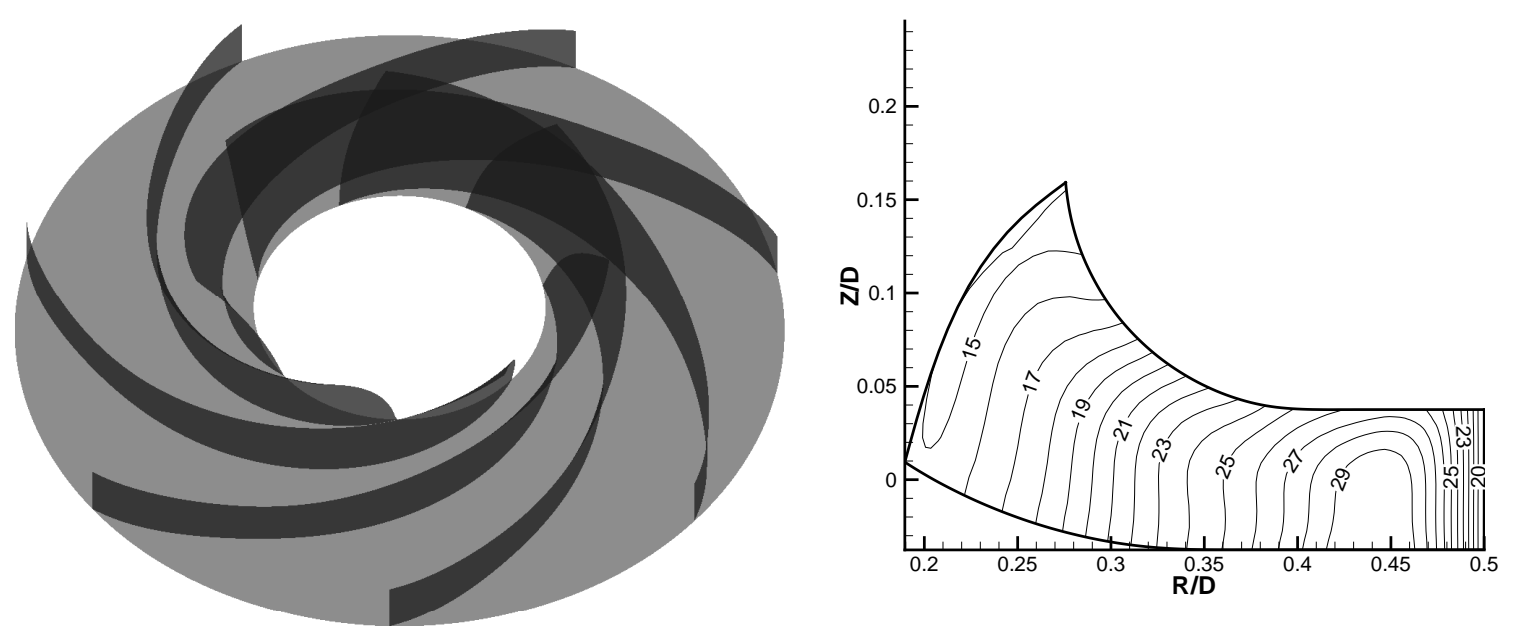

Figure 3.13: SHF impeller inverse-design case 1: the inversely-designed impeller (left) and blade angle distribution $\beta(m, s)$ in the meridional plane (right).
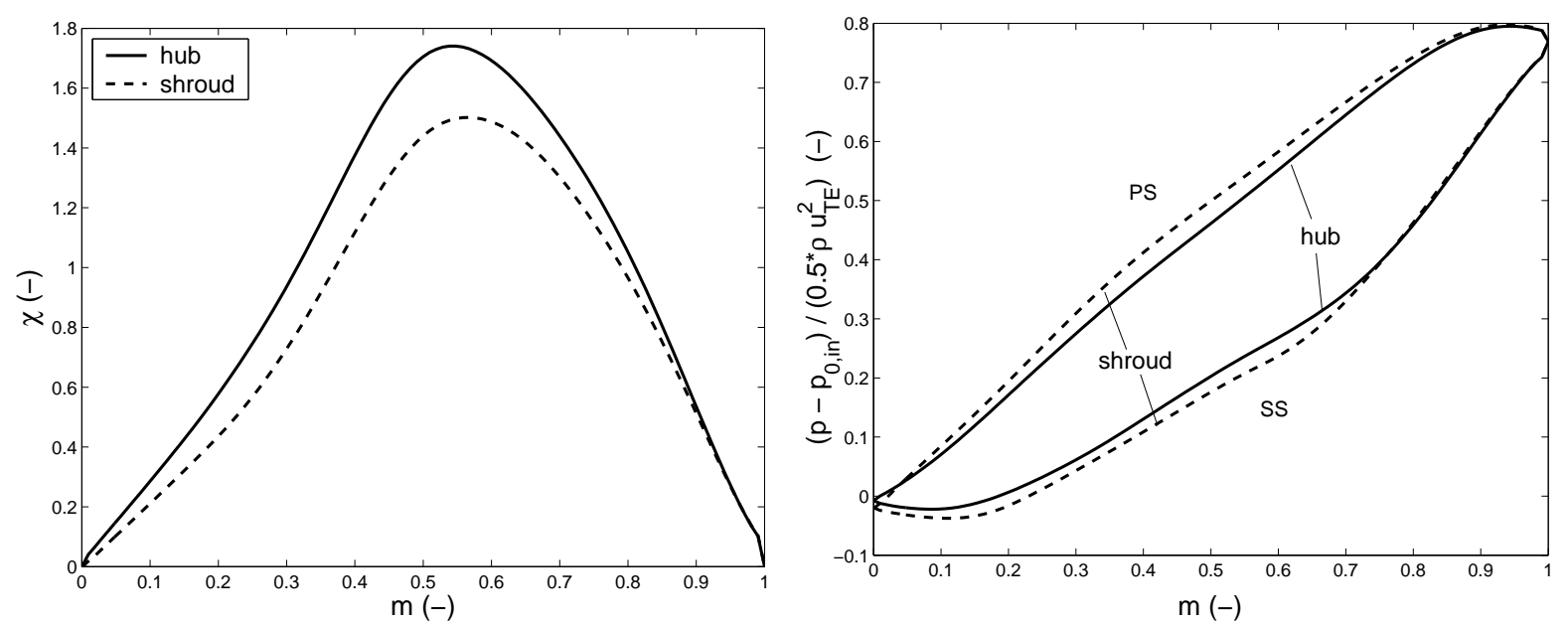

Figure 3.14: SHF impeller inverse-design case 1: the velocity loading on the blades (left) and the non-dimensional pressure distribution (right). PS indicates the pressure side and SS the suction side. 
$\kappa_{i}=2.65 \cdot 10^{-2}$. The boundary layer loss coefficient is slightly higher than that for the original design, $\zeta=2.63 \cdot 10^{-2}$, versus $\zeta=2.50 \cdot 10^{-2}$ for the original design.

Clearly, this first inverse-design has a better cavitation performance than the original geometry. However, the velocity loading is considerable higher than that for the original design. Therefore, a second inverse-design is investigated in the next section, in order to reduce the maximum in the velocity loading. 


\subsubsection{SHF impeller inverse-design case 2}

A second inverse-design is carried out for the SHF impeller with the aim of reducing the maximum in the velocity loading. For this purpose, a mean-swirl distribution is selected which is close to a linear build-up. This mean-swirl distribution is constant from hub to shroud and it is shown in Fig. 3.15, where it is compared to the meanswirl distribution used for case 1. Such a mean-swirl distribution is obtained by using a spline interpolation [35] based on values of the non-dimensional mean-swirl distribution $f(m)=\left\{r \bar{v}_{\theta}(m)\right\} /\left\{r_{t e} \bar{v}_{\theta, t e}\right\}$ given at user specified points from leading to trailing edge, as is shown in table 3.6. The numerical parameters for this inverse-design calculation are equal to the ones presented in table 3.5 for the fine mesh; 59 iterations are needed to achieve convergence.

Table 3.6: Non-dimensional mean-swirl values for the SHF impeller design case 2.

\begin{tabular}{|c|c|c|c|c|}
\hline$m$ & 0.0 & 0.2 & 0.8 & 1.0 \\
\hline$f(m)$ & 0.0 & 0.15 & 0.85 & 1.0 \\
\hline
\end{tabular}
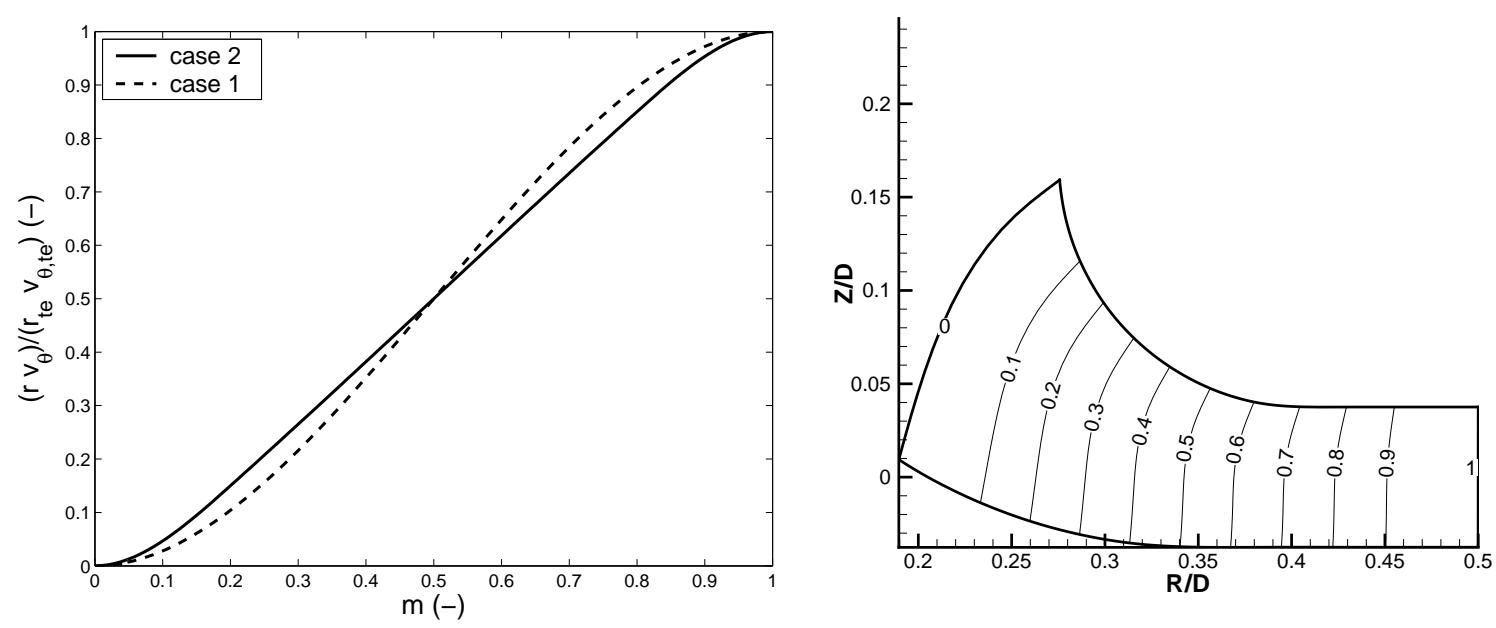

Figure 3.15: SHF impeller inverse-design case 2: the normalized mean-swirl distribution as a function of the meridional length (left) and as a contour plot in the meridional plane (right).

The newly designed impeller and the corresponding blade angle distribution are shown in Fig. 3.16. The blade angles are between $15^{\circ}$ and $27^{\circ}$ degrees. The maximum of the blade angle is lower than for the first inverse-design case. The variation in blade angle from leading to trailing edge is also smaller when compared to the first inverse-design case, but still more pronounced than for the original design. The blade angles at the leading and trailing edge are similar to those of the first inverse-design case, which is hardly surprising since both designs have a prescribed zero incidence at the leading edge, and a prescribed pump head at the trailing edge. The velocity loading and the pressure distribution at the hub and the shroud are shown in Fig. 3.17. It is observed that the maximum in the velocity loading is reduced and a more evenly distributed velocity and pressure loading have been obtained. 

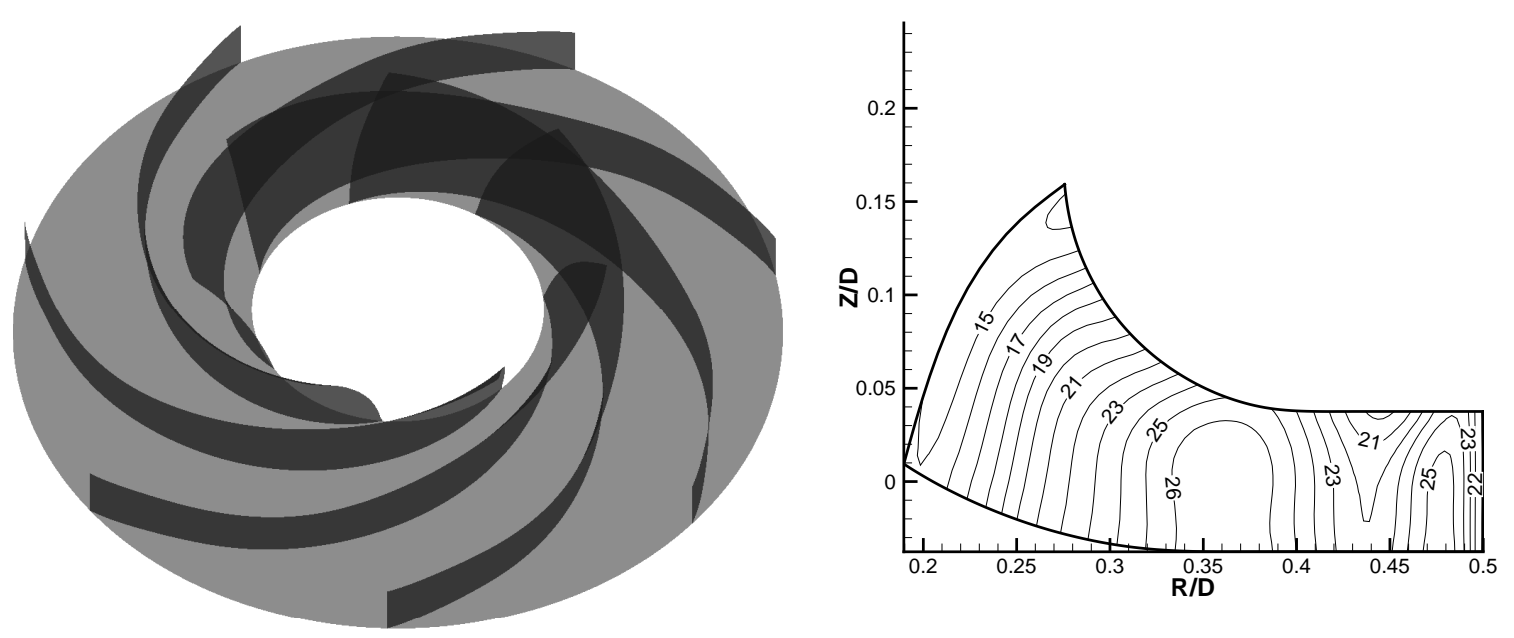

Figure 3.16: SHF impeller inverse-design case 2: the inversely-designed impeller (left) and blade angle distribution $\beta(m, s)$ in the meridional plane (right).

The maximum velocity loading coefficient is $\chi_{\max }=1.44$. This is considerably lower than the first inverse-design case, for which $\chi_{\max }=1.74$, but still slightly higher than the original design, for which $\chi_{\max }=1.34$. The cavitation inception coefficient is again much lower than the original design and comparable to the default inverse-design, $\kappa_{i}=0.52$. $10^{-2}$. The boundary layer loss coefficient for this second inverse-design is $\zeta=2.43 \cdot 10^{-2}$, which is slightly lower than that for the original $\left(\zeta=2.50 \cdot 10^{-2}\right)$ and inverse-design case $1\left(\zeta=2.63 \cdot 10^{-2}\right)$.

\subsubsection{Comparison of SHF impeller designs}

In this section the three designs are compared and discussed. Firstly, the blade geometries are compared. To this end the blade shapes and the blade angles at the hub are compared in Fig. 3.18. The largest differences are observed at the leading edge, where the original blade angle at the hub differs significantly from that of the two inverse-designs. This is in accordance with the conclusion drawn earlier that the original design does not satisfy the zero-incidence condition at the leading edge. The blade angles at the trailing edge are comparable for all designs, due to the designs having the same pump head.

In Fig. 3.19 the relative velocity profiles of the designs are compared at the intersection of the blade with the hub and with the shroud. The influence of the non-zero incidence for the original design can clearly be observed in the distribution near the leading edge. The lowest relative velocities and the largest decelerations occur for the first inverse-design case, at the pressure side. This means that this design would experience back-flow first when the flow rate is lowered gradually from the design flow rate $Q_{d}$, which clearly is disadvantageous. Furthermore, the difference in relative velocity between pressure and suction side is largest for the first inverse-design case, as has already been concluded from the large maximum in the velocity loading. 

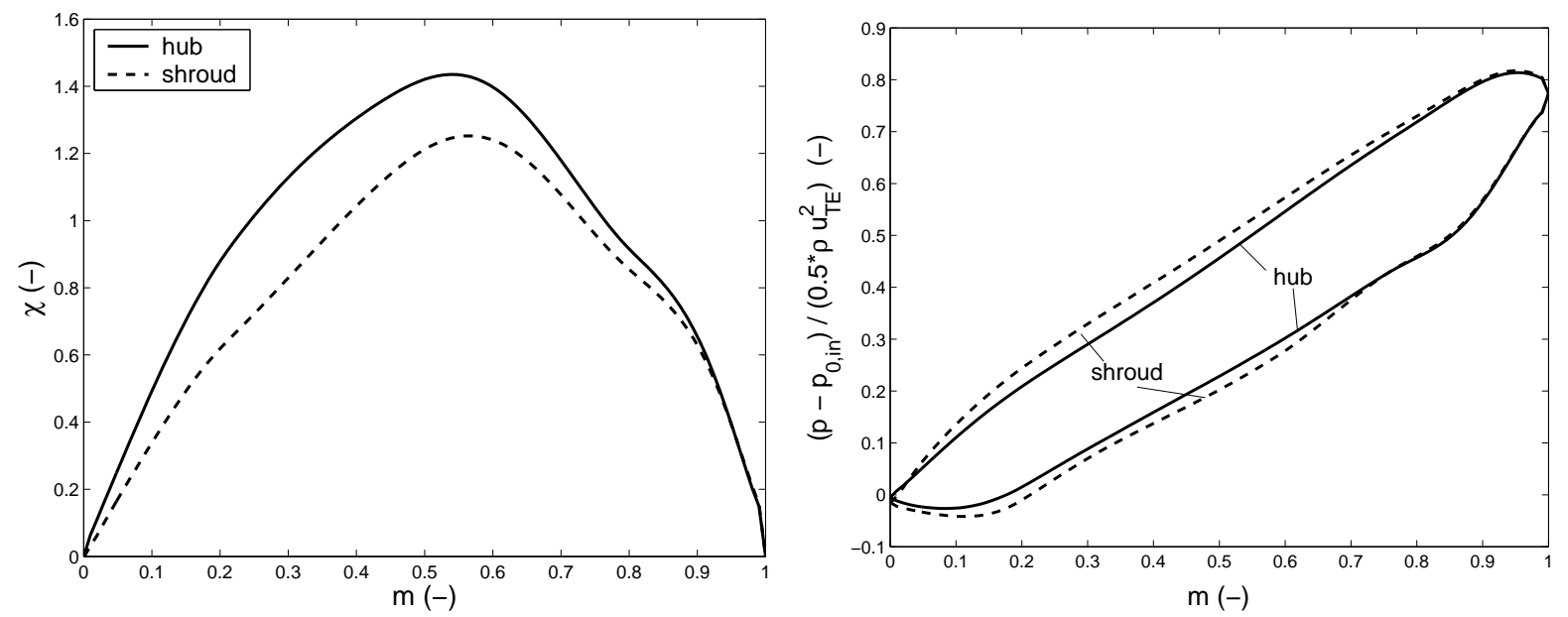

Figure 3.17: SHF impeller inverse-design case 2: the velocity loading on the blades (left) and the non-dimensional pressure distribution (right). PS indicates the pressure side and SS the suction side.

Table 3.7: Performance coefficients for the SHF impeller designs.

\begin{tabular}{|c||c|c|c|}
\hline case & $\zeta\left(10^{-2}\right)$ & $\kappa_{i}\left(10^{-2}\right)$ & $\chi_{\max }$ \\
\hline original & 2.50 & 2.65 & 1.34 \\
\hline inverse-design case 1 & 2.63 & 0.47 & 1.74 \\
\hline inverse-design case 2 & 2.43 & 0.52 & 1.44 \\
\hline
\end{tabular}

The performance coefficients are compared in table 3.7. The loss coefficient $\zeta$ for the three designs are comparable, with the second inverse-design case having the best value. The losses are comparable since the largest velocities in the designs considered are of similar magnitude (see Fig. 3.19) with the largest velocities mainly determining the boundary layer losses in the impellers (see Eqn. (2.27)).

The cavitation inception behavior for the original geometry is clearly not as good as for the inverse-designs, caused by the non-zero zero incidence at the leading edge. The first and second inverse-design cases have a similar cavitation performance, due to the prescribed zero incidence at the leading edge. This accomplishment is a major advantage of the inverse-design method.

The maximum velocity loading coefficient for the first inverse-design case is very high when compared to the original and the second inverse-design case.

Summarizing, the boundary layer losses are similar for all designs considered. The original design has a relative low maximum velocity loading, but has a poor cavitation inception performance due to the non-zero incidence angle at the leading edge. The first inverse-design case has a much better cavitation performance due to the zero-incidence at the leading edge. However, the first inverse-design case has a relative high maximum velocity loading and will be the first design to suffer from back-flow when the flow rate is lower than the design flow rate. Finally, the second inverse-design case has both a good cavitation performance and a good velocity loading performance. Therefore, it is concluded that the second inverse-design case is the best design considered. This also 

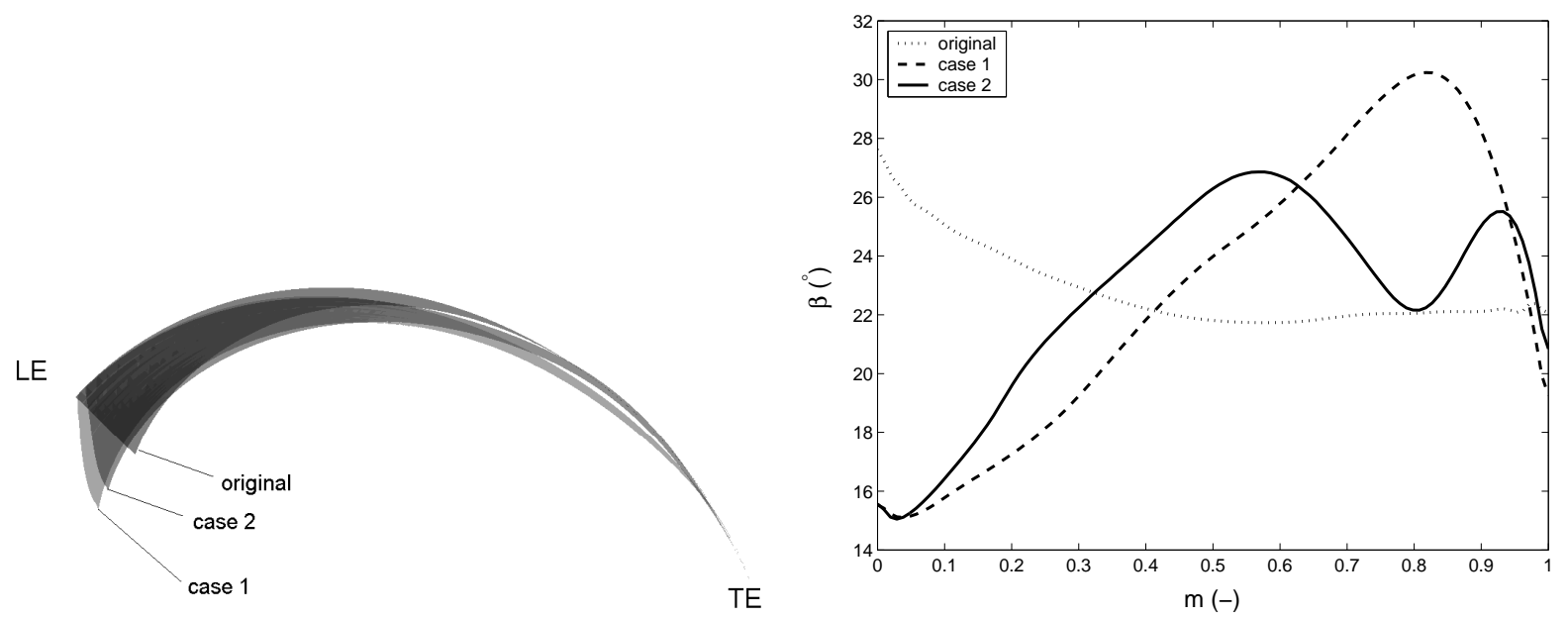

Figure 3.18: Comparison of SHF impeller designs: top view of the blade shapes (left) and blade angle distribution at the hub $\beta(m, 0)$ (right).
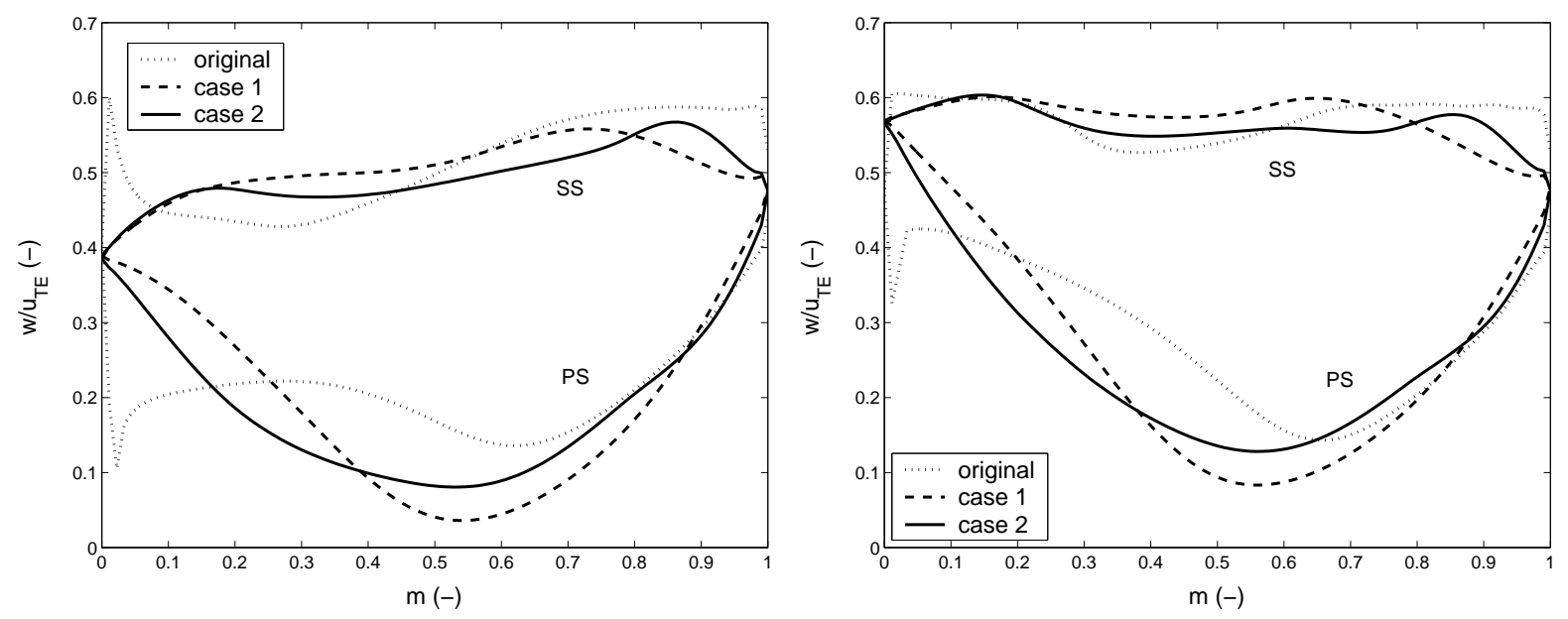

Figure 3.19: Comparison of SHF impeller designs: the relative velocity profiles at the hub (left) and at the shroud (right). PS indicates the pressure side and SS the suction side.

shows that specific design objectives can be achieved by an appropriate choice of the mean-swirl distribution. Further adjustment of the mean-swirl distribution $r \bar{v}_{\theta}(m)$ could be considered to reduce the maximum velocity loading $\chi_{\max }$ further, while maintaining a good cavitation inception number $\kappa_{i}$. 


\subsection{Inverse-design of mixed-flow impeller blades}

In the preceding section the inverse-design of a 3D radial impeller has been presented. Here we apply the inverse-design method to the redesign of a mixed-flow impeller. The original impeller is a design from Flowserve [25]. Firstly, the original design will be presented. Two inverse-design cases are discussed subsequently, followed by the comparison of the designs.

\subsubsection{Mixed-flow impeller original design}

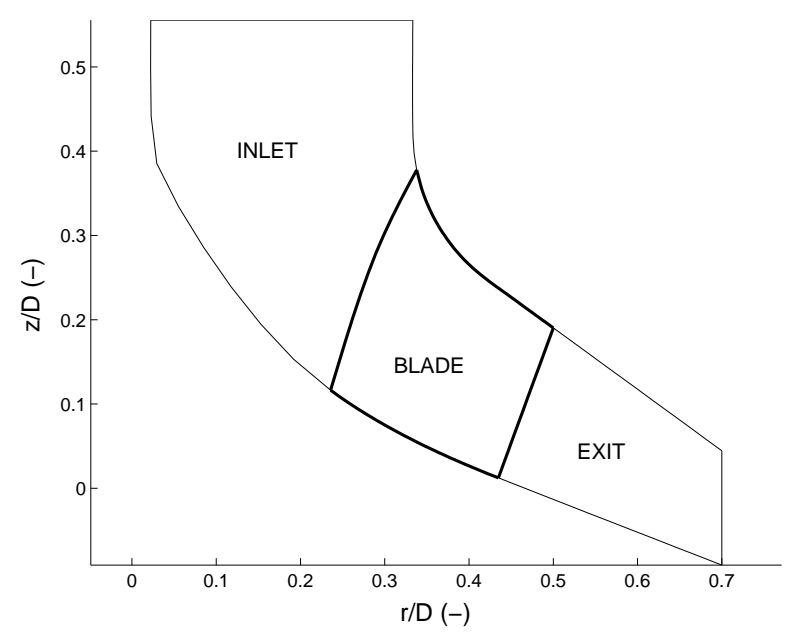

Figure 3.20: Meridional shape of the mixed-flow impeller.

The meridional shape of the impeller blades is shown in Fig. 3.20. The impeller has 5 blades and is displayed in Fig. 3.21, along with the blade angle distribution. The blade angles are rather constant for the original design, varying between $18^{\circ}$ and $21^{\circ}$. The blade has a considerable stacking angle distribution at the trailing edge of $14^{\circ}$ from hub to shroud. The design parameters for the impeller are listed in table 3.8. These parameters have been determined from a direct computation of the impeller, with zero-thickness blades. The velocity loading and the pressure distribution are shown in Fig. 3.22. The

Table 3.8: Dimensionless characteristics of the mixed-flow impeller at design point.

\begin{tabular}{|c|c|c|c|}
\hline$Z$ & $\phi$ & $\psi$ & $N_{s}$ \\
\hline 5 & 0.31 & 0.35 & 1.24 \\
\hline
\end{tabular}

blades clearly do satisfy the zero-incidence condition at the leading edge, as shown by the large gradients in the velocity loading and pressure distributions near the leading edge, especially at the hub. The pressure difference between pressure and suction side is larger at the shroud, which seems to be in contradiction with the velocity loading, which is larger at the hub. The reason for this is that the average relative velocity is higher at the shroud than at the hub, as can be seen from the relative velocity profiles in Fig. 3.30, which will later be discussed in more detail. 

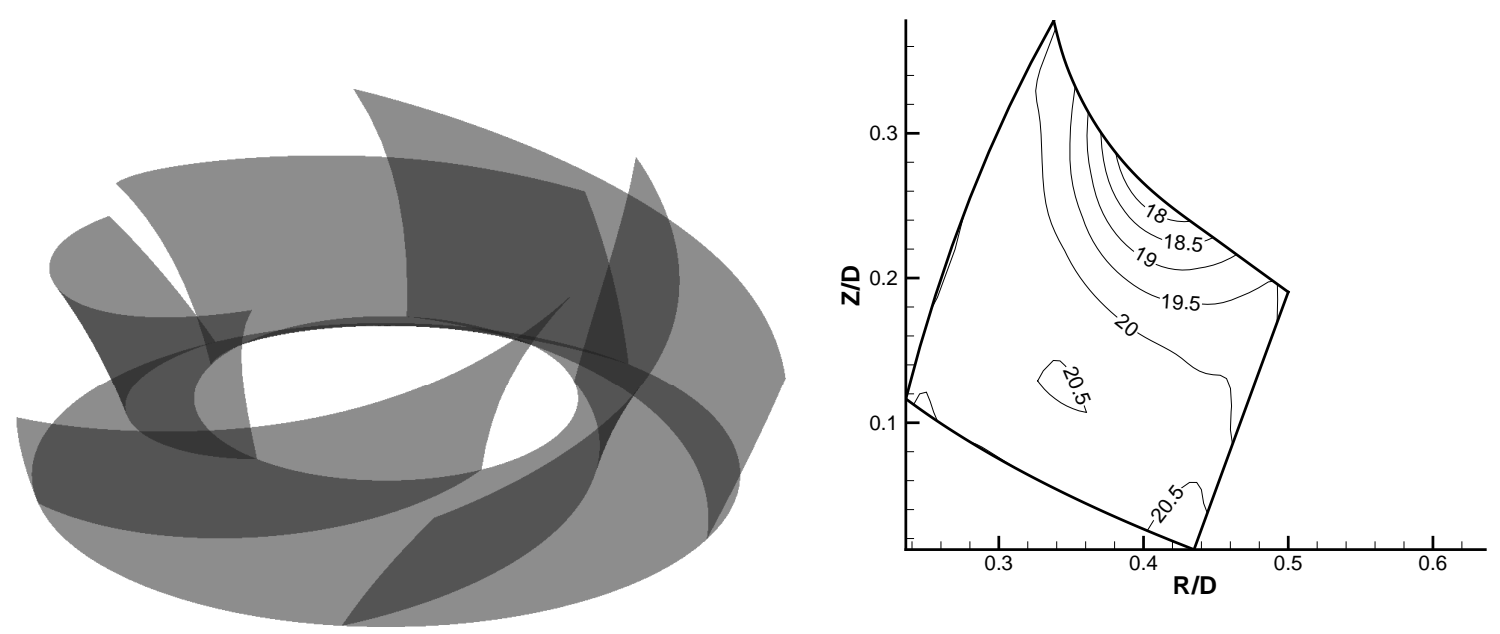

Figure 3.21: Mixed-flow impeller original design: the mixed-flow impeller (left) and its blade angle distribution $\beta(m, s)$ in the meridional plane (right).
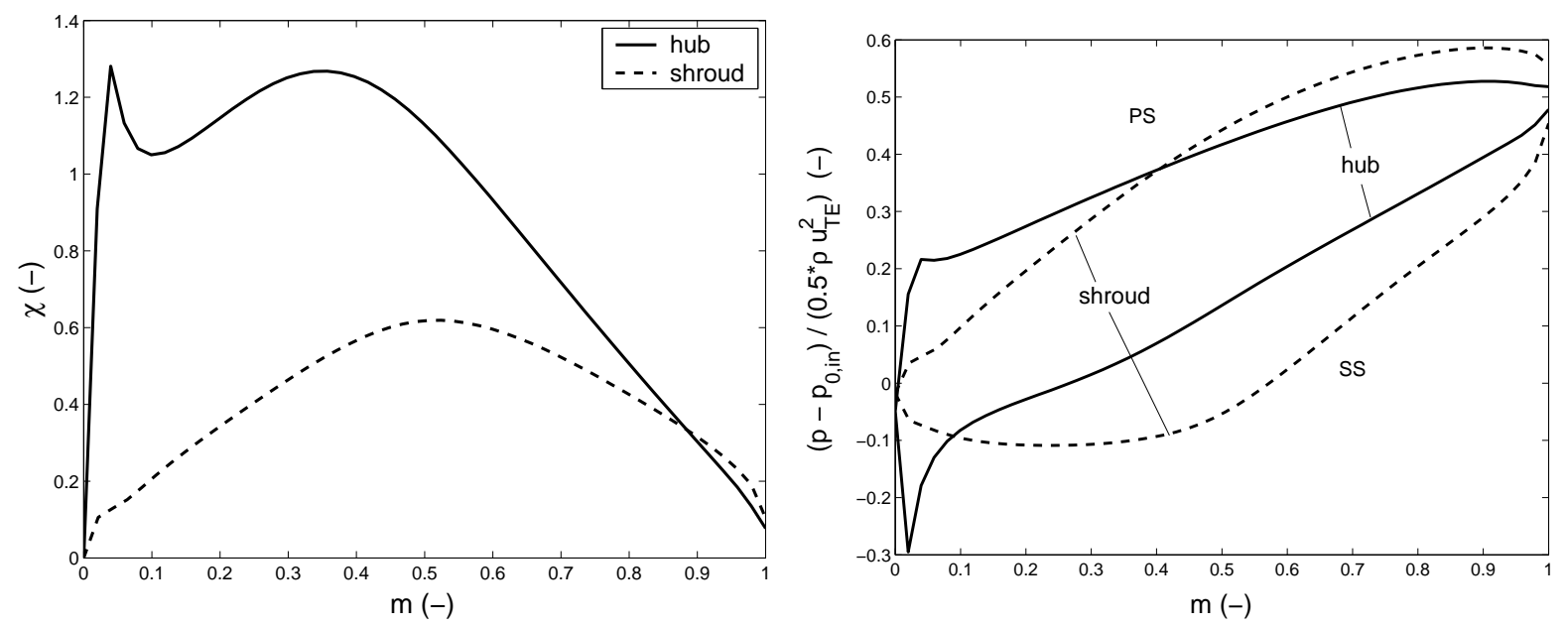

Figure 3.22: Mixed-flow impeller original design: the velocity loading on the blades (left) and the non-dimensional pressure distribution (right). PS indicates the pressure side and SS the suction side. 
The maximum for the velocity loading coefficient, defined in Eqn. (3.54), is rather large, $\chi_{\max }=1.28$. The cavitation inception coefficient, given by Eqn. (1.10), for the original impeller is $\kappa_{i}=3.69 \cdot 10^{-2}$ and the boundary layer loss coefficient, defined by Eqn. $(2.28)$, is $\zeta=3.73 \cdot 10^{-2}$.

\subsubsection{Mixed-flow impeller inverse-design case 1}

For the first inverse-design of this impeller the modified mean-swirl distribution, from section 3.5.3 has been selected. The advantage of this mean-swirl distribution, which is constant from hub to shroud, is that it has a more or less evenly distributed loading over the blade.

The function values that determine the interpolation, based on cubic splines, are given in table 3.9. The corresponding dimensionless swirl distribution $f_{1}\left(m_{1}\right)$ is displayed in Fig. 3.23, where it is compared to the mean-swirl distribution of the original geometry obtained from a direct computation. The function $f_{2}\left(m_{2}\right)$, also given in table 3.9, will be used for the loading at the hub in the next section.
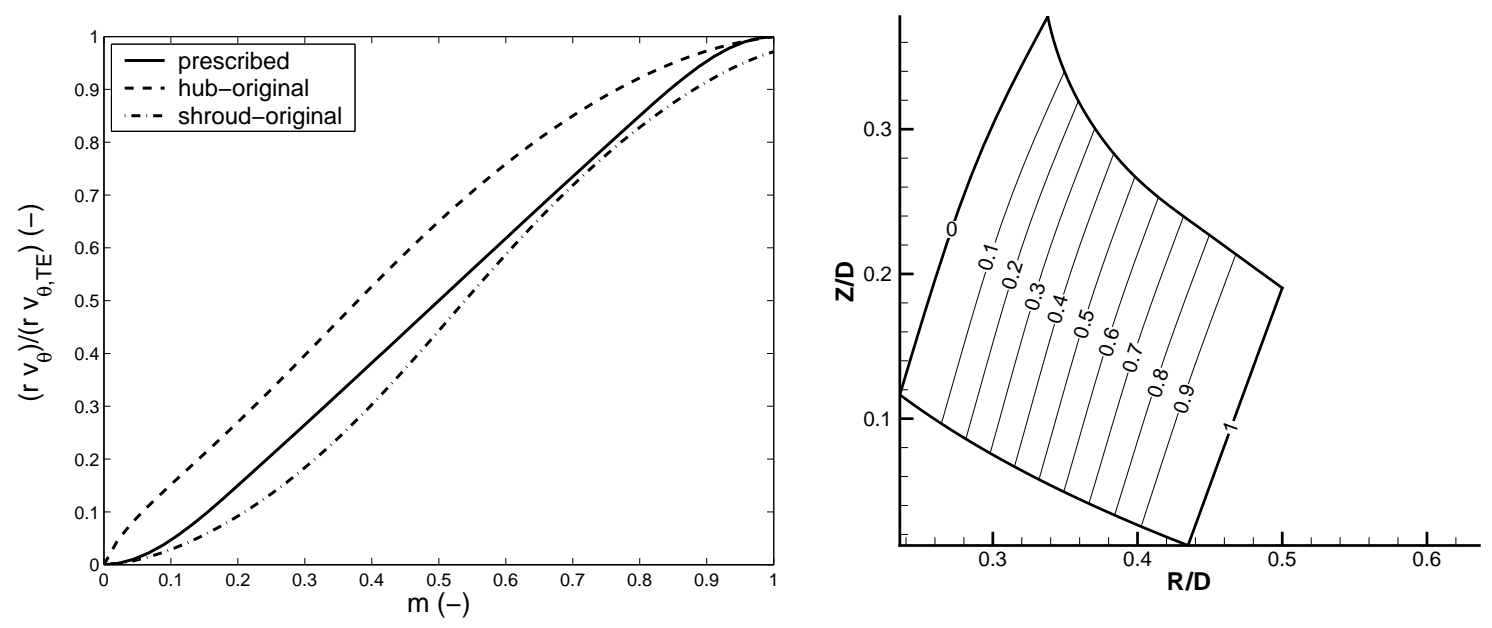

Figure 3.23: Mixed-flow impeller inverse-design case 1: the prescribed mean-swirl distribution compared with the computed profiles for the original impeller (left). A contour plot of the prescribed mean-swirl distribution in the meridional plane is shown on the right.

Table 3.9: Mean-swirl function values for the mixed flow impeller computations.

\begin{tabular}{|c|c||c|c|}
\hline$m_{1}$ & $f_{1}\left(m_{1}\right)$ & $m_{2}$ & $f_{2}\left(m_{2}\right)$ \\
\hline 0.0 & 0.0 & 0.0 & 0.0 \\
\hline 0.2 & 0.15 & 0.4 & 0.2 \\
\hline 0.8 & 0.85 & 1.0 & 1.0 \\
\hline 1.0 & 1.0 & - & - \\
\hline
\end{tabular}

The considerable stacking at the trailing edge and the low number of blades (5) make it a difficult inverse-design computation. Therefore, a a small value of the under-relaxation 

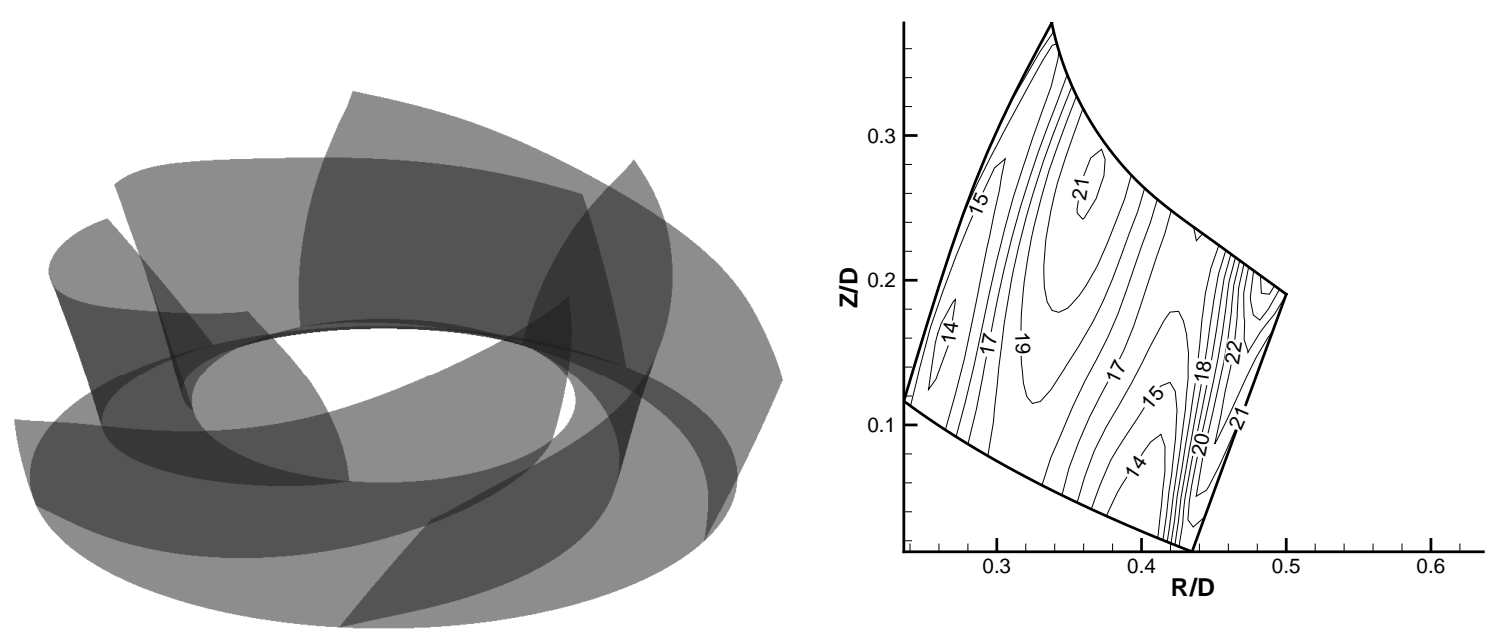

Figure 3.24: Mixed-flow impeller inverse-design case 1: the inversely-designed impeller (left) and its blade angle distribution $\beta(m, s)$ in the meridional plane (right).

coefficient has to be selected. The employed numerical parameters are listed in table 3.10.

Table 3.10: Numerical parameters for the mixed flow impeller.

\begin{tabular}{|c|c|c|c||c|c|c|}
\hline ni & nj & nk & $n_{\text {tets }}$ & $\omega$ & $\Delta \theta_{\text {lim }}(\mathrm{rad})$ & $\epsilon$ \\
\hline 75 & 35 & 13 & 204,750 & 0.1 & 0.5 & $1.10^{-3}$ \\
\hline
\end{tabular}

Starting from an initial estimate with a constant blade angle of $18^{\circ}, 31$ iterations are needed to obtain convergence. The resulting impeller is shown in Fig. 3.24 along with the corresponding blade angle distribution $\beta(m, s)$. The blade angles for this inversedesign are mainly between $13^{\circ}$ and $23^{\circ}$. The variations in curvature between leading and trailing edge are clearly much larger than for the original design (compare Fig. 3.24 with Fig. 3.21).

The velocity loading and the pressure distribution for the inverse-design case 1 are displayed in Fig. 3.25. As is the case for the original geometry, the velocity loading at the shroud is lower than that at the hub, although the difference is smaller for this design. No large gradients are observed near the leading edge, indicating that this geometry indeed results in the prescribed zero-incidence at the leading edge, unlike the original geometry.

The maximum for the velocity loading coefficient, $\chi_{\max }=0.85$, is significantly reduced in comparison with the original geometry, for which $\chi_{\max }=1.28$. The cavitation inception coefficient for the inversely-designed impeller is found at $\kappa_{i}=1.60 \cdot 10^{-2}$, which is also a significant improvement (for the original impeller $\kappa_{i}=3.69 \cdot 10^{-2}$ ) due to the better flow conditions at the leading edge. The boundary layer loss coefficient for the inverse-design is $\zeta=4.30 \cdot 10^{-2}$, which is somewhat higher than for the original geometry, for which $\zeta=3.73 \cdot 10^{-2}$. 

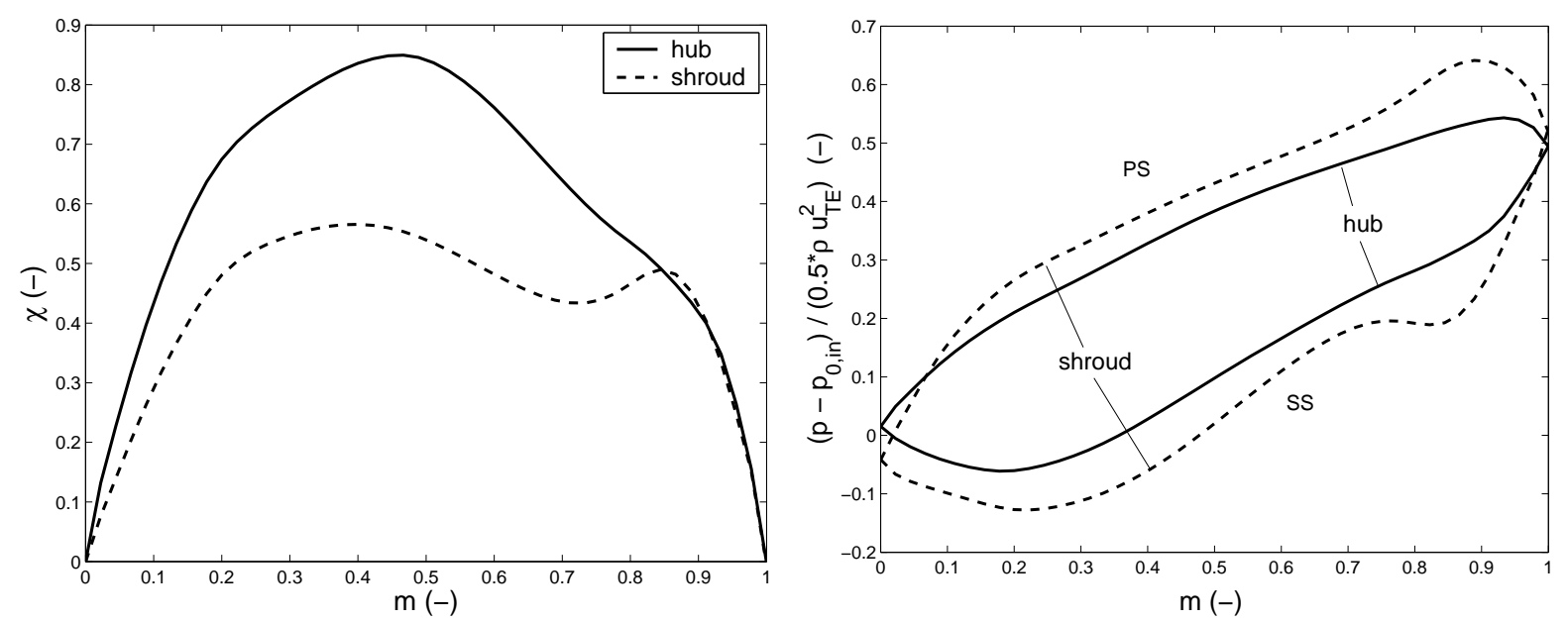

Figure 3.25: Mixed-flow impeller inverse-design case 1: the velocity loading on the blades (left) and the non-dimensional pressure distribution (right). PS indicates the pressure side and SS the suction side.

\subsubsection{Mixed-flow impeller inverse-design case 2}

For the second inverse-design case an attempt is made to further reduce the maximum velocity $\chi_{\max }$ on the blades. Since the maximum velocity loading occurs at the hub, the loading function for the shroud is maintained (given by $f_{1}$ ) and the loading function for the hub is altered to $f_{2}$, i.e. the loading is increased towards the trailing edge, see also Fig. 3.26 and table 3.9. 42 iterations are needed to achieve convergence, using the same numerical parameters as for the first inverse-design case (see table 3.10).

The inversely-designed impeller and the associated blade angle distribution are given in Fig. 3.27. The blade angles are largely between $12^{\circ}$ and $25^{\circ}$, which is comparable with the distribution obtained in the first inverse-design case. The blade angles at the shroud are very similar for both inverse-design cases, but the blade angle distribution at the hub shows marked differences, as can be seen by comparing Fig. 3.24 and Fig. 3.27. The hub blade contour is longer than for the first inverse-design and the blade angles are smaller (small blade angles lead to long blades). These differences at the hub are a direct consequence of the modification of the mean-swirl distribution at the hub for the second inverse-design case. Shifting the loading towards the trailing edge generally leads to longer blade contours.

The velocity loading and the pressure distribution at the hub and the shroud are shown in Fig. 3.28. It can be concluded that the maximum velocity loading is indeed reduced, to $\chi_{\max }=0.76$, compared to the original impeller for which $\chi_{\max }=1.28$ and the first inverse-design case for which $\chi_{\max }=0.85$. However, this comes at the cost of a considerably larger boundary layer loss coefficient, $\zeta=4.91 \cdot 10^{-2}$, compared to $\zeta=3.73 \cdot 10^{-2}$ for the original impeller and $\zeta=4.30 \cdot 10^{-2}$ for the first inverse-design case. Furthermore, for this second design the pressure build up at the hub is shifted more towards the trailing edge, and this leads to a higher minimum pressure and therefore a lower value for the cavitation inception coefficient, $\kappa_{i}=1.39 \cdot 10^{-2}$, compared to the original geometry for which $\kappa_{i}=3.69 \cdot 10^{-2}$ and the first inverse-design case for which 

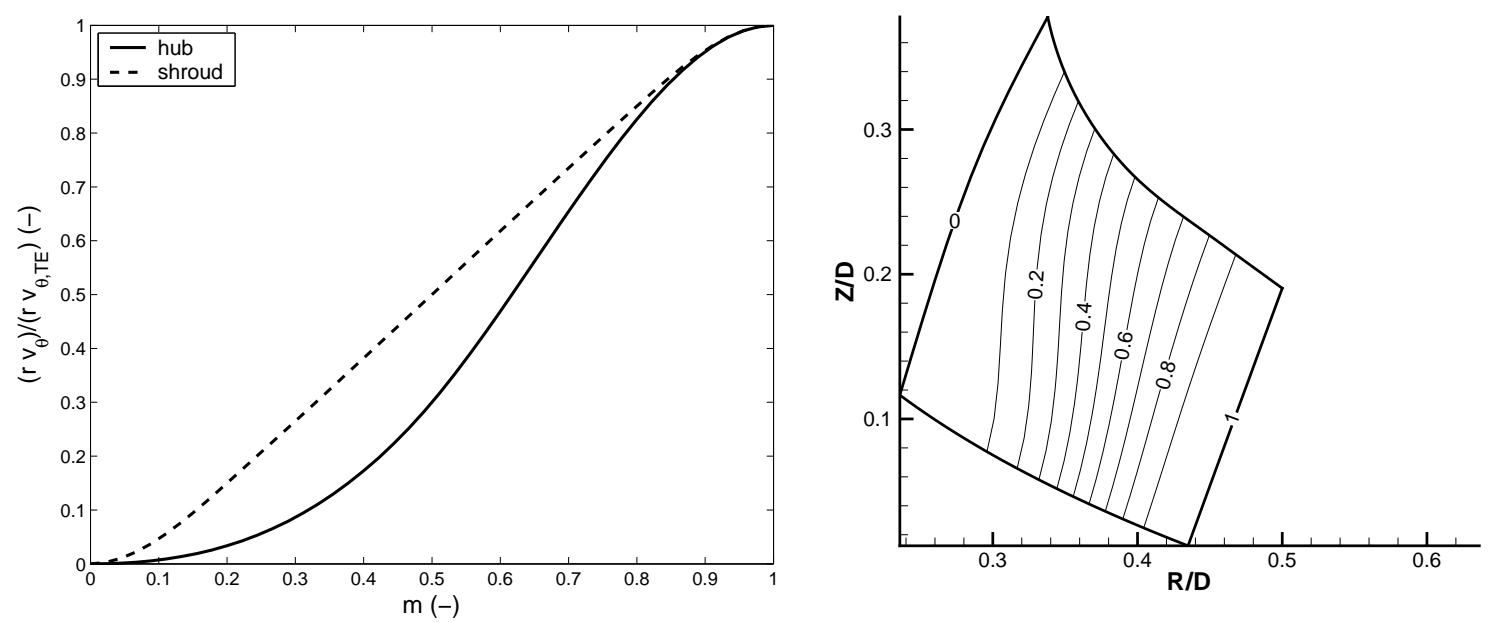

Figure 3.26: Mixed-flow impeller inverse-design case 2: the modified prescribed mean-swirl distribution. Shown as function of the meridional length (left) and as a contour plot in the meridional plane (right).

$\kappa_{i}=1.60 \cdot 10^{-2}$. The second design has a higher loading near the trailing edge at the hub and it is up to a designer to assess whether this is desirable. In this case it was selected to reduce the maximum velocity loading, which has been accomplished successfully. 

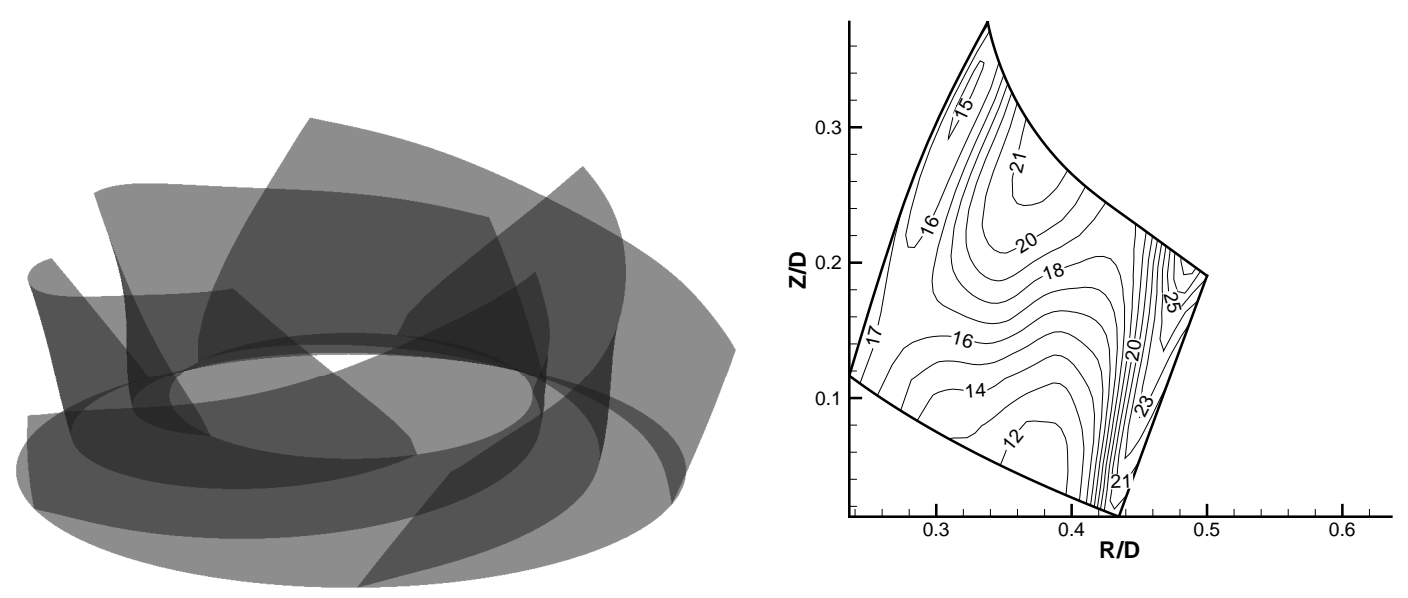

Figure 3.27: Mixed-flow impeller inverse-design case 2: the inversely-designed impeller (left) and its blade angle distribution $\beta(m, s)$ in the meridional plane (right).
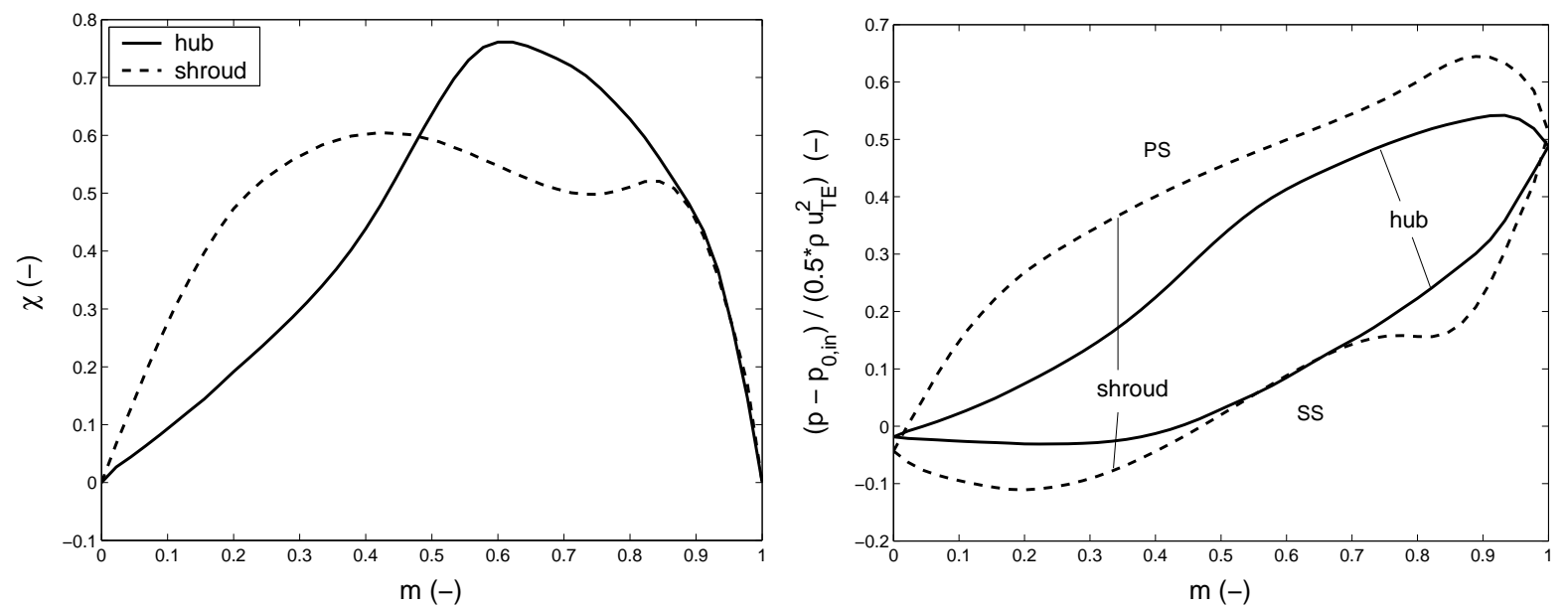

Figure 3.28: Mixed-flow impeller inverse-design case 2: the velocity loading on the blades (left) and the non-dimensional pressure distribution (right). PS indicates the pressure side and SS the suction side. 
Table 3.11: Performance coefficients for the mixed-flow impeller designs.

\begin{tabular}{|c||c|c|c|}
\hline case & $\zeta\left(10^{-2}\right)$ & $\kappa_{i}\left(10^{-2}\right)$ & $\chi_{\max }$ \\
\hline original & 3.73 & 3.69 & 1.28 \\
\hline inverse-design case 1 & 4.30 & 1.60 & 0.85 \\
\hline inverse-design case 2 & 4.91 & 1.39 & 0.76 \\
\hline
\end{tabular}

\subsubsection{Comparison of mixed-flow impeller designs}
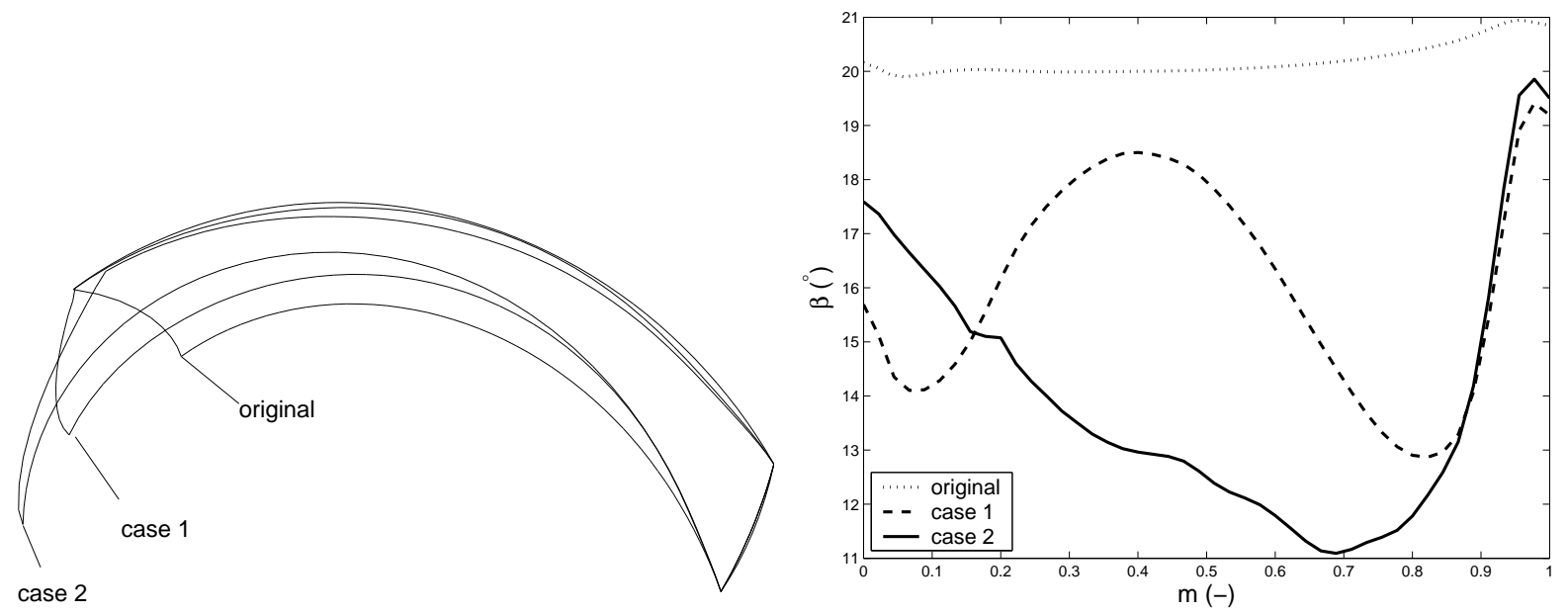

Figure 3.29: Comparison of mixed-flow impeller designs: top view of the blade contours (left) and blade angle distribution at the hub (right).

In this section the three designs are compared and discussed. In Fig. 3.29 the blades and the distribution of the blade angle along the blade-hub intersection are compared. The blade angle distribution at the hub is shown, since the differences in geometry of the designs are most pronounced here. The hub contour is longest for inverse-design case 2 (also seen from the small value of the blade angles) and shortest for the original design (also seen from the large value of the blade angles). The original impeller has an almost uniform blade angle distribution at the trailing edge across the span (from hub to shroud), whereas the inverse-designs have a high trailing edge blade angle at the shroud and a low trailing edge blade angle at the hub, see also the meridional blade angle distributions in Figs. 3.21, 3.24 and 3.27. This results in all designs having the same pump head.

The distribution of the relative velocity at the blade-hub and the blade-shroud intersection are compared in Fig. 3.30. The differences between the inverse-design cases 1 and 2 are small at the shroud, due to the identical prescribed mean-swirl distribution at the shroud. At the hub, case 2 differs from case 1 due to the shift in prescribed mean-swirl distribution. The effect of shifting the hub loading more towards the trailing edge for case 2 can clearly be seen in the relative velocity profile at the blade-hub intersection.

In table 3.11 the performance parameters for the three designs are listed. The cavitation inception performance is best for inverse-design case 2, and worst for the original geometry, which does not have zero-incidence at the leading edge. 

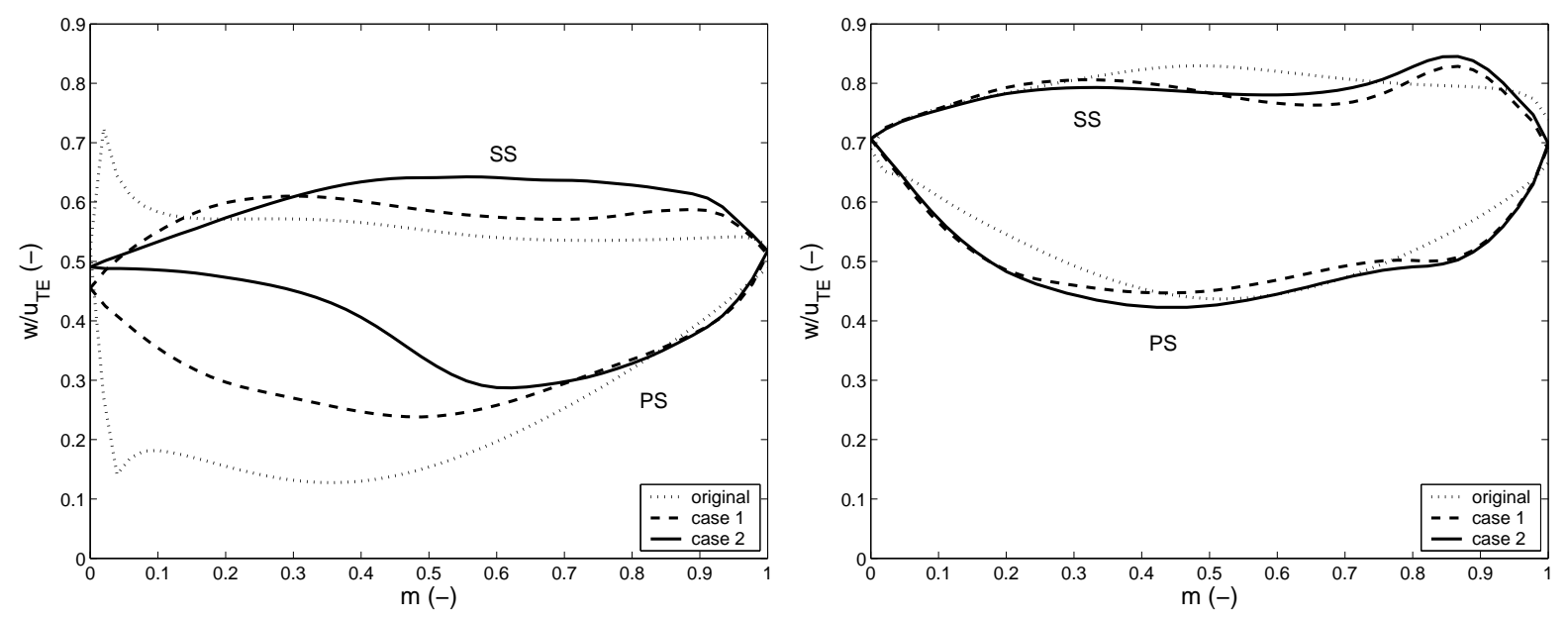

Figure 3.30: Comparison of mixed-flow impeller designs: distribution of the relative velocity at the hub (left) and at the shroud (right). PS indicates the pressure side and SS the suction side.

The boundary layer losses are lowest for the original design, and highest for the second inverse-design case, due to the relative large relative velocities near the trailing edge (see Fig. 3.30) and the larger surface area of the blades.

The two inversely-designed impellers form an improvement when compared to the original impeller, due to the much lower value of the cavitation inception coefficient $\kappa_{i}$ and the maximum velocity loading coefficient $\chi_{\max }$. The drawback of somewhat higher boundary layer losses is considered to be less significant. Whether inverse-design case 1 is preferred over design case 2, depends on the application. For example, if cavitation prevention is important inverse-design case 2 is preferred and if boundary layer losses are considered to be more important, inverse-design case 1 will be selected. 


\subsection{Alternative specification of loading}

In preceding sections inverse-design calculations have been presented that are based on prescribed mean-swirl distributions. It has been illustrated how the prescribed mean-swirl distribution can be changed in order to obtain a front or aft loaded blade. By using a different loading, this design process may be made easier to tune.

Not only mean-swirl distributions can be employed, but also other ways to prescribe the loading can be utilized that are more frequently used in industry. In the next sections several alternative prescriptions of the loading are presented. However, it has to be stressed that the method in itself does not change. All these alternative specifications are converted into a mean-swirl distribution, which is then used as a periodic boundary condition to solve the Laplace equation, as is illustrated by the numerical algorithm shown in Fig. 3.2. More details about the use of different specifications of the blade loading can be found in $[5]$.

\subsubsection{Derivative of the mean-swirl distribution}

The first alternative prescription of the loading that will be discussed is that of the derivative of the mean-swirl distribution, i.e. $\partial\left(r \bar{v}_{\theta}\right) / \partial m$. It is often more convenient to adjust certain loadings through the derivative of the mean-swirl rather than through the mean-swirl itself. If the derivative is prescribed, the mean-swirl distribution can be obtained by straightforward integration.

$$
r \bar{v}_{\theta}=\int_{m=0}^{m} \frac{\partial r v_{\theta}}{\partial m} d m
$$

In the method the prescribed derivative is dimensionless, and therefore the obtained meanswirl distribution $r \bar{v}_{\theta}$ has to be scaled such that the prescribed pump head is obtained. This is done by employing the condition at the trailing edge given by Eqn. (3.4), so we use the quantity $\left(r \bar{v}_{\theta}\right) /\left(r \bar{v}_{\theta}\right)_{t e}$, with $\left(r \bar{v}_{\theta}\right)_{t e}=g H / \Omega$.

\subsubsection{Velocity difference distribution}

In industry the blade loadings are frequently formulated as the velocity difference. Here the velocity difference is formulated in terms of the magnitude of the velocity difference in the direction of the blade contour $l, w_{l}^{p s}-w_{l}^{s s}$. Here it is assumed that the loading is constant from hub to shroud, thus independent of the span-wise direction $s$. Therefore, it suffices to focus on the difference in velocity in the direction of the blade contour $l$ only. For blades of zero thickness $u_{n}^{p s}=u_{n}^{s s}$ and we can write this velocity difference distribution as

$$
w_{l}^{p s}-w_{l}^{s s}=v_{l}^{p s}-v_{l}^{s s}=\frac{\partial\left(\phi^{p s}-\phi^{s s}\right)}{\partial x_{l}}
$$

At the blade surface the impenetrability condition holds and therefore $v_{n}^{p s}=u_{n}$ and $v_{n}^{s s}=u_{n}$. The blade angle gives a relation between the blade contour direction $l$ and 
the meridional coordinate $m$, as has been shown in Eqn. (3.9). Using this equation, the following relation is obtained for the velocity difference distribution

$$
w_{l}^{p s}-w_{l}^{s s}=\sin \beta \frac{\partial\left(\phi^{p s}-\phi^{s s}\right)}{\partial x_{m}}
$$

Using Eqn. (3.1), the velocity difference distribution can be written as function of the mean-swirl distribution

$$
\Delta w=w_{l}^{p s}-w_{l}^{s s}=\left\|\boldsymbol{w}^{p s}-\boldsymbol{w}^{s s}\right\|=\frac{2 \pi \sin \beta}{Z} \frac{\partial r \bar{v}_{\theta}}{\partial x_{m}}
$$

where $\beta$ is defined with respect to the circumferential direction (see Eqn. (1.6)).

The velocity difference distribution $\Delta w$ is to be prescribed in a dimensionless form. From this velocity distribution the mean-swirl distribution needs to be derived. During the iterative adjustment of the blade shape, the blade angle distribution $\beta$ changes. Therefore the mean-swirl distribution has to be calculated at the start of each iteration $i$ based on the blade angle from the previous iteration $i-1$. The dimensionless velocity difference distribution is converted to the derivative of the mean-swirl distribution using Eqn. (3.59) and the mean-swirl distribution is then obtained by integration, as described in Eqn. (3.56) and scaling with Eqn. (3.4).

Finally, it is has to be noted that the velocity difference distribution presented here is not the same as the velocity loading $\chi$, which has been used in preceding sections and is defined in Eqn. (3.54).

\subsubsection{Application of a velocity difference distribution}

In the preceding sections two alternative ways to prescribe the loading are discussed, i.e. the derivative of the mean-swirl distribution and the velocity difference distribution $\Delta w$. Here an inverse-design is presented based on a prescribed velocity difference distribution. The case considered is the SHF case, which has been treated in section 3.5. The prescribed velocity difference distribution used is constant from hub to shroud and is loaded towards the trailing edge (aft loaded), as is shown in Fig. 3.31 and in table 3.12. The resulting inversely-designed impeller and corresponding blade angle distribution are displayed in Fig. 3.32. When compared to the previous designs from section 3.5, the inversely-designed impeller is not markedly different from the earlier inverse-designs. The blade is somewhat longer, due to the shift in loading towards the trailing edge.

Table 3.12: Prescribed dimensionless velocity difference values $\Delta w(m)$ used for the inversedesign of the SHF impeller.

\begin{tabular}{|c||c|c|c|c|}
\hline$m$ & 0.0 & 0.5 & 0.8 & 1.0 \\
\hline$\Delta w(m)$ & 0.0 & 0.5 & 0.5 & 0.0 \\
\hline
\end{tabular}

The velocity loading coefficient and the pressure distribution on the blade are shown in Fig. 3.33, and it is clear that the loading has been shifted towards the trailing edge. The maximum for the velocity loading coefficient is $\chi_{\max }=1.43$. The cavitation inception 

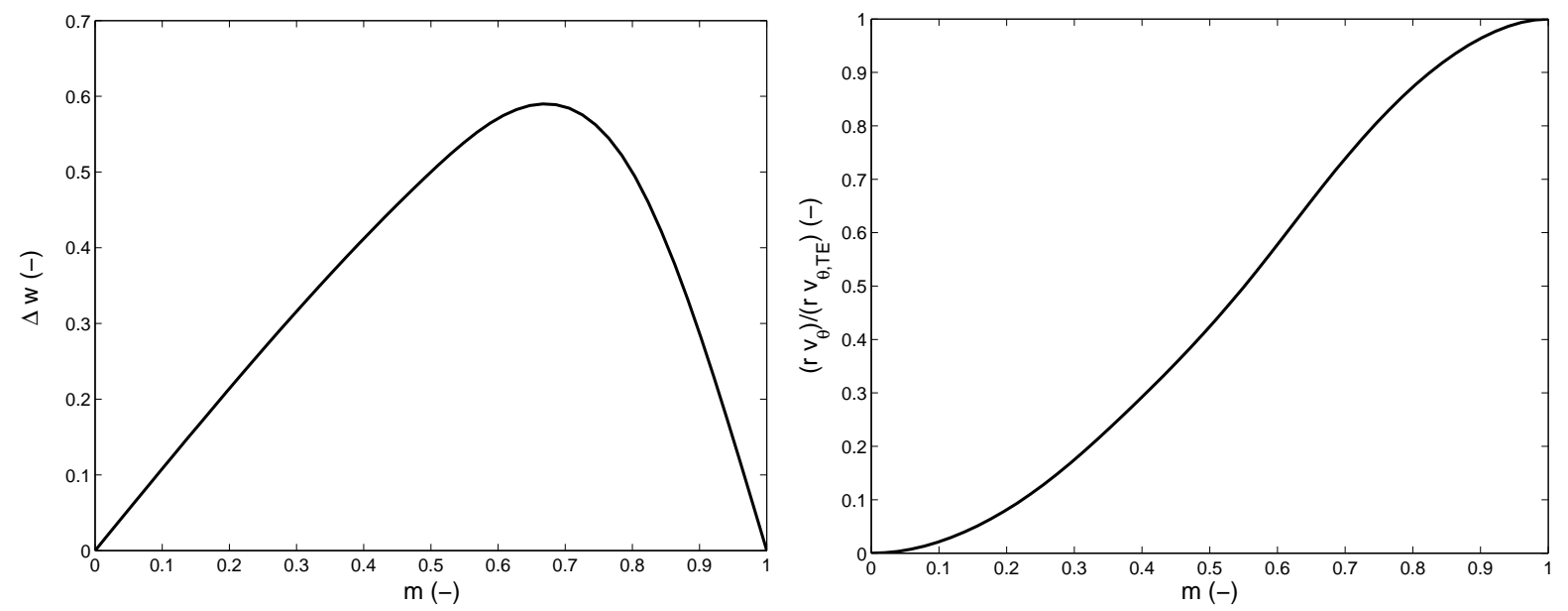

Figure 3.31: Velocity difference distribution for the inverse-design of the SHF impeller: the prescribed dimensionless velocity difference as a function of the meridional distance $m$ (left) and the corresponding mean-swirl distribution of the inverse-design (right).

coefficient for this inversely-designed impeller is $\kappa_{i}=0.44 \cdot 10^{-2}$ and the boundary layer loss coefficient is, $\zeta=3.05 \cdot 10^{-2}$. These values are comparable to those of the inversedesigns presented in section 3.5 (see table 3.7).

It is concluded that besides using a mean-swirl distribution as loading function for the inverse-design method, it is also possible to use the (dimensionless) velocity difference as loading function. In practice, there is little difference between using the velocity difference or the derivative of the mean-swirl distribution as loading function, unless there is a large variation in blade angle from leading to trailing edge (see Eqn. (3.58). For such large differences, the method will become less robust due to the implicit influence of the blade angle on the velocity loading (see Eqn. (3.59)), and therefore it is preferred in most cases to use a derivative or mean-swirl distribution itself as loading function instead, although the velocity difference distribution is physically more meaningful. 

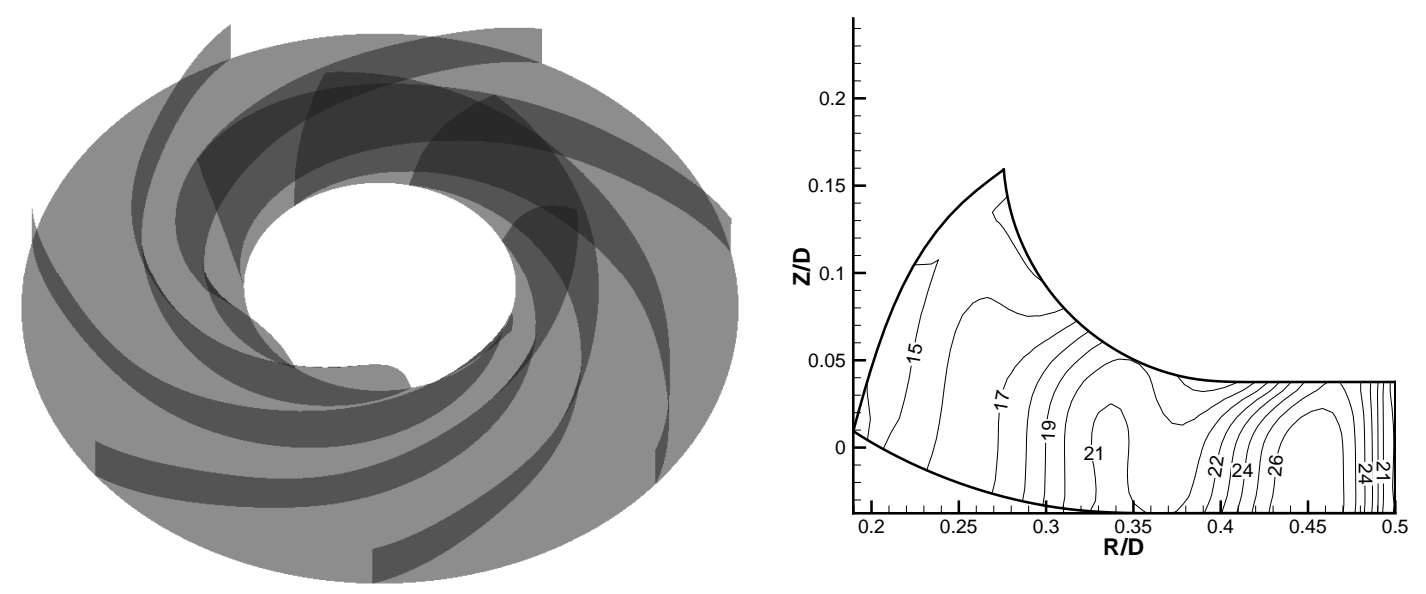

Figure 3.32: Inverse-design of the SHF impeller using a prescribed velocity difference distribution: the inversely designed impeller (left) and blade angle distribution in the meridional plane (right).
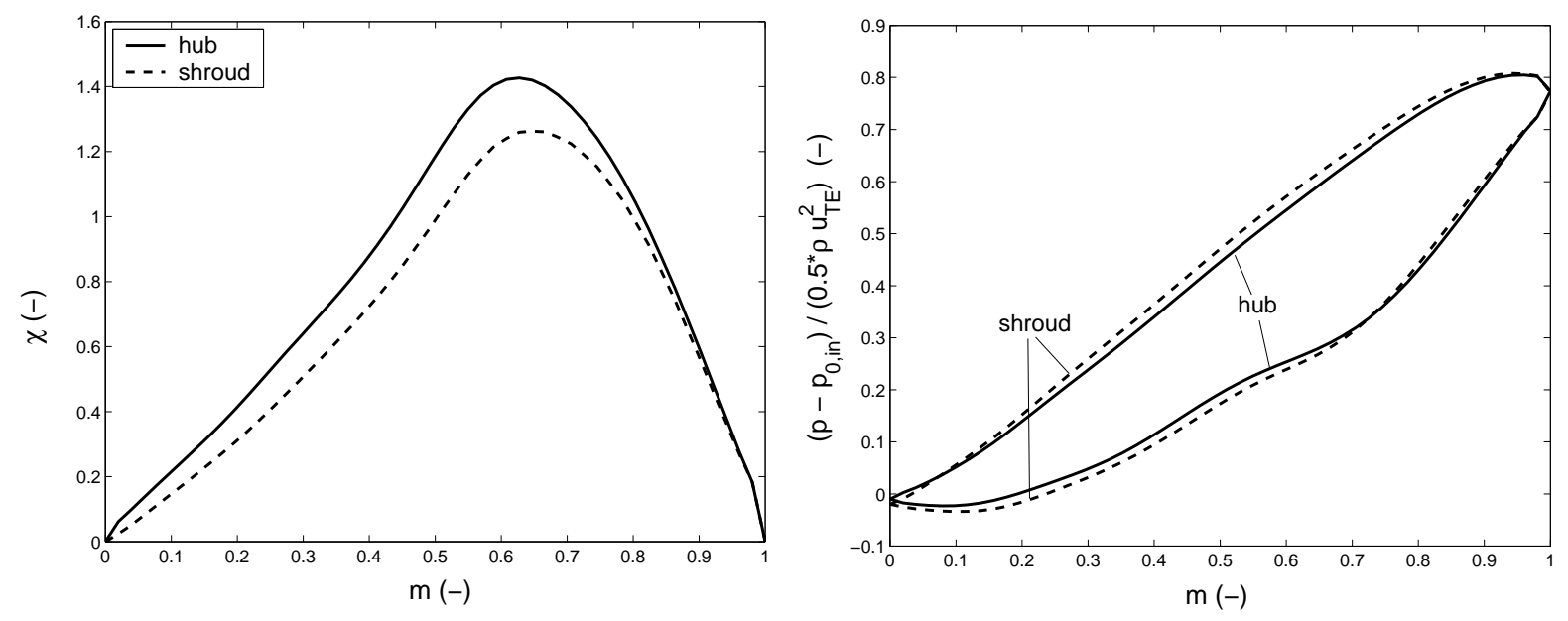

Figure 3.33: Inverse-design of the SHF impeller using a prescribed velocity difference distribution: the velocity loading on the blades (left) and the non-dimensional pressure distribution (right). PS indicates the pressure side and SS the suction side. 


\subsection{Discussion and conclusions}

An inverse-design method for centrifugal impeller blades has been presented. The main strength of the method is that desirable performance conditions, like the desired pump head and the zero-incidence condition at the leading edge can easily be prescribed via the mean-swirl distribution. By changing the build up of the mean-swirl distribution the loading of the blades can be modified as well.

In the inverse-design method the blade curvature is changed, whereas other geometrical quantities remain unchanged, like the number of blades on the impeller, the stacking condition at the trailing edge and the meridional geometry. The design choices of these geometrical features partly determine the quality of the inverse designs.

The method has been verified by two cases in section 3.4, for which the resulting geometry is known and can be compared to the inversely-computed geometry. The method is robust and it is first-order accurate as is shown in 3.4.3, caused by the relative low accuracy of the relative velocities near the blade surface when using the SPR-method (see section 2.4.4).

The developed inverse-design method has been applied to the radial SHF impeller in section 3.5. For the first case a default mean-swirl distribution is applied. The inverselydesigned impeller satisfies the zero-incidence condition at the leading edge (unlike the original geometry), leading to a significant improvement in the cavitation inception behavior. However, the maximum velocity loading coefficient $\chi_{\max }$ is relatively high. The second inverse-design presented has been performed in order to reduce this maximum velocity loading on the blade. By using a mean-swirl distribution, which has a more or less constant blade loading from leading to trailing edge, the maximum loading coefficient has been reduced successfully.

Furthermore, an inverse computation of a mixed flow impeller, with a considerable stacking at the trailing edge, is presented in section 3.6. For the first inverse-design case considered, the loading is applied which was used successfully for the radial impeller. This again leads to an impeller with a relatively low maximum velocity loading and a zero-incidence at the leading edge. A second inverse computation is considered for the mixed-flow impeller in order to further reduce the velocity loading. This second inverselydesigned impeller has more favorable cavitation properties, but the boundary layer losses and the maximum in the velocity loading are less favorable than for the first inverselydesigned impeller.

In section 3.7 different ways of prescribing a loading distribution are presented, i.e. through the derivative of the mean-swirl distribution or through a velocity difference distribution. A prescribed loading using the latter approach is employed to the inversedesign of the SHF impeller, showing its capabilities.

The method has been developed for blades of zero thickness. In reality the blades have a certain thickness, which results in a blockage effect that influences the velocity and the pressure distribution on the blade, especially near the leading edge. A prescribed tangential blade thickness distribution, as has been proposed by Zangeneh [80], can be used to describe the blockage effect. However, for backward curved blades the round shape of the blade nose can not be presented accurately, making this approach limited in its use, especially in conjunction with analyzes of cavitation inception characteristics. For the 
implementation of a prescribed thickness distribution in two-dimensional configurations in the current method the reader is referred to Bijleveld [5].

The impeller performance parameters, like the velocity loading, the cavitation characteristics and the boundary layer losses can be improved through the inverse-design method by changing the prescribed mean-swirl distributions. In this way certain aspects of the blade geometry can be adjusted. However, an exact control over the resulting blade angles and thus the blade shape is not obtained. Generally, front loaded blades lead to shorter blades with low relative velocities. These impellers have the advantage of relative low losses, but back flow will occur sooner for these impellers when operating at lower flow rates. Aft loaded blades, for which the loading is shifted more towards the trailing edge, lead to longer blades. 



\section{CHAPTER 4}

\section{Optimization Method}

In the preceding chapters it has been pointed out that the design of turbomachines is a complicated task. In the inverse-design method presented in chapter 3, the impeller blade curvature is determined such that a prescribed performance function is achieved. Instead of prescribing a desirable performance function, one can also parameterize the geometry and optimize the performance, given in the form of a cost function. Through this cost function the designer can specify desirable and undesirable aspects of designs. This procedure of parameterization, specifying a cost function and solving the minimization problem is called an optimization method.

Optimization methods for turbomachines have become popular in the last decade, made possible by the advances in computing power. A flow analysis is usually carried out to determine the value of the cost function for a specified geometry. Typical performance parameters that are included in the cost function are head, efficiency and cavitation characteristics. The cost function is then optimized using an optimization algorithm.

The contents of this chapter are as follows. Firstly, a general introduction to optimization methods is given in section 4.1. This includes a literature overview of optimization methods for turbomachines. Subsequently, the optimization algorithm used to solve the minimization problem is discussed in section 4.2. The employed method, i.e. the method of Differential Evolution (DE), is verified by application of the method to three analytical cost functions. In section 4.3 the optimization of a radial pump impeller is presented. The parameterization, cost function and the optimization results are discussed and a comparison is made of the original and the optimized geometries. Furthermore, additional optimizations have been performed to investigate the influence of both parameterization and formulation of the cost function on the results. In section 4.4 a hybrid method is presented, which is labeled inverse-optimization. The mean-swirl distribution, which is used as input for the inverse-design method, presented in chapter 3, is parameterized for this purpose. A description is given of this parameterization, the employed cost function and the resulting optimized inverse-design. Finally, the utilized optimization approaches are discussed in section 4.5. 


\subsection{Introduction to optimization}

In the optimization of a turbomachine the performance properties are optimized by changing relevant parameters. The performance properties are usually expressed in the form of a cost or fitness function $F$. The parameters are frequently presented as a parameter vector $\boldsymbol{x}$. When the parameters are changed, the cost function $F(\boldsymbol{x})$ changes as well. Optimization methods are usually formulated as minimization problems. Therefore, the goal of the optimization is to find the parameter vector $\boldsymbol{x}$ for which the object function $F(\boldsymbol{x})$ is minimal. An appropriate algorithm is to be selected to solve such a minimization problem. The general procedure of optimization can be summarized as follows

- Parameterization: selection of a parameter vector $\boldsymbol{x}$ and possible constraints on $\boldsymbol{x}$, for example a bounded range.

- Cost function: how to evaluate the fitness $F(\boldsymbol{x})$ of each individual geometry parameterized by $\boldsymbol{x}$. For this purpose, turbomachine characteristics are included in an appropriate fashion in this object function.

- Optimization algorithm: selection of a suitable algorithm to solve the minimization problem

Optimization methods can be either single-point or multi-point, meaning that the performance and thus the cost function is considered for a single operating point or for multiple operating points, respectively. Since the performance of turbomachines is not only important at design condition but also at off-design conditions, a multi-point optimization method is proposed, such that the performance is optimized for a certain operating range rather than a single operating point only.

Several objectives can be considered for optimization, dealing with pump head, efficiency and cavitation characteristics. There are two approaches that can be used to carry out an optimization in which multiple objectives are considered. Firstly, all the objectives can be added to a single so-called lumped cost function using weight factors. For this approach a single optimum is found. Secondly, a multi-objective optimization can be considered in which each objective is optimized and a so-called Pareto front of optimal solutions is obtained [1]. This approach thus yields multiple optima and the designer has to select one of these geometries employing certain criteria. In the present study the lumped cost-function approach is used. An important step in this approach is to choose the weight factors for the different objectives such that all have a desired contribution to the lumped cost function.

\subsubsection{Literature overview}

In this section an overview is given on the application of optimization methods for turbomachinery design.

Pierret et al. [53] developed an optimization method for turbomachinery blades using an artificial neural network as approximate model. They applied their method to redesign a compressor, the so-called NASA rotor 67 , by optimizing the efficiency at three different 
operating conditions. Their analysis consists of both a flow analysis and a structural analysis. Jang et al. [41] optimized the blade sweep in a transonic axial compressor rotor (NASA rotor 47) using a response surface method and a three-dimensional Navier-Stokes analysis to increase its efficiency. Burguburu et al. [8, 9] presented an optimization method based on a quasi three-dimensional Navier-Stokes analysis. They employed the conjugate-gradient method (and a genetic algorithm for verification [8]) to find the optimum of a cost function, which was based on efficiency. They applied their method to the design of both compressor rotor blades and turbine stator blades. Talya et al. [65] developed a multi-disciplinary optimization method for film-cooled gas turbines, using a quasi three-dimensional Navier-Stokes method. The cost function was based on the blade temperature and a gradient method was used to find the optimum.

A hybrid method, considering the optimization of an inverse-design method has been presented by Peng et al. [51]. In their research the mean-swirl distribution is parameterized and both efficiency and cavitation objectives are considered in the cost function. The conjugate gradient method was used as optimization algorithm. Yiu et al. [79] also optimized the mean-swirl distribution for an inviscid inverse-design method. For each resulting inverse-design a viscous flow analysis is performed using a three-dimensional Navier-Stokes method. The cost function consists of the viscous losses computed with this method. They investigated the effect of parameterization, cost function and optimization algorithm, i.e. the simplex method and the alternating-variables method. This approach was further extended by Tiow et al. [67], who used simulated annealing as optimization algorithm. They successfully applied the method to the design of a compressor rotor and a turbine stator.

The above overview is by no means complete, but gives an overview on the available literature on optimization methods in the field of turbomachinery. An overview on recent work on optimization methods can be found in [37].

\subsubsection{Requirements for the optimization algorithm}

It is important to select an optimization algorithm, that satisfies the requirements for optimization. The following requirements are relevant for the optimizations considered in this chapter. The algorithm

- has to be able to deal with non-differentiable objective functions;

- should yield the global minimum;

- has a limited number of parameters, which can be selected easily.

Adjoint or gradient-based methods are frequently used optimization methods. These methods search for the optimum of the cost function $F\left(x_{1}, x_{2}, ..\right)$ by making use of the gradient of the cost function $\partial F / \partial x_{1}, \partial F / \partial x_{2}, \ldots$ These methods are usually relatively fast, but they are not able to deal with cost functions that are non-differentiable or have several local optima. An overview on gradient-based optimization methods can be found in [54]. 
Several global methods have been investigated for possible use, including Simulated Annealing, Genetic Algorithm, Pattern Search and Differential Evolution. For an overview of such methods, see for example [59]. The Differential Evolution method was selected, since it was easy to implement and showed promising results, as will be demonstrated in section 4.2. An optimization of a radial pump impeller using a genetic algorithm has been carried out by Antonakis [3] as starting point in the present project.

\subsection{Differential Evolution}

In this section the Differential Evolution (DE) method is presented, which is used as optimization algorithm in the present investigation. The method has been developed by Storn and Price [64]. More extensive overviews of Differential Evolution and its applications are given, for example, in $[33,55]$. The DE method can be classified as a member of the family of evolutionary algorithms. Other algorithms that belong to this family are Genetic Algorithms (GA), Evolutionary Strategies (ES) and Evolutionary Programming (EP). Five important steps can be distinguished in DE, i.e. initialization, mutation, recombination, selection and termination. The method satisfies the requirements formulated in section 4.1.2.

The parameter vector $\boldsymbol{x}$ is $N$-dimensional, where $N$ is the number of parameters in the optimization process. Evolutionary algorithms are population based. The size of a population is denoted by $P$. For a population member $p$ the $n^{\text {th }}$ parameter is indicated by $x_{n}^{p}$ (with $n=1 \ldots N$ and $p=1 \ldots P$ ).

\section{Initialization}

Initialize a random population $X$ with a population size $P$. Each individual parameter vector $\boldsymbol{x}^{p}$, with $p$ the index in the population $P$, has a specific set of values for the design parameters and a corresponding value of the cost function $F\left(\boldsymbol{x}^{\boldsymbol{p}}\right)$.

\section{Mutation}

Create a new population $Y$ of population size $P$ by mutating individuals from the previous population $X$ for each parameter $n$.

$$
\boldsymbol{y}^{\boldsymbol{p}}=\boldsymbol{x}^{\boldsymbol{a}}+T\left(\boldsymbol{x}^{\boldsymbol{b}}-\boldsymbol{x}^{c}\right)
$$

where $\boldsymbol{x}^{\boldsymbol{a}}, \boldsymbol{x}^{\boldsymbol{b}}$ and $\boldsymbol{x}^{\boldsymbol{c}}$ are randomly selected but different individuals of the population $(p=1 \ldots P, p \neq a \neq b \neq c)$ and $T$ is the mutation coefficient with $0<T<1$.

\section{Recombination}

For each component $y_{n}^{p}$ of the new parameter vectors $\boldsymbol{y}^{\boldsymbol{p}}$ a random number $q$ is generated $(0<q<1)$. If $q \leq C$, then the newly mutated individual component is introduced in the new population, otherwise the previous individual component $x_{n}^{p}$ is retained in the new population. $C$ is the recombination coefficient with $0<C<1$. 


\section{Selection}

Determine the cost function for each individual $\boldsymbol{y}^{\boldsymbol{p}}$ in the new population $Y$ and compare its value $F\left(\boldsymbol{y}^{p}\right)$ to the cost function of the corresponding individual in the previous population $F\left(\boldsymbol{x}^{\boldsymbol{p}}\right)$. If $F\left(\boldsymbol{y}^{\boldsymbol{p}}\right)<F\left(\boldsymbol{x}^{\boldsymbol{p}}\right)$, the new individual replaces the previous one in the population $X$, otherwise the previous individual is retained. This procedure is designated as selection based on the greedy criterion [64].

\section{Termination}

Repeat the mutation, recombination and selection for a fixed number of generations $G$. Analyze the iterative data to see whether sufficient convergence is obtained.

Note that geometrical parameters that are of integer value, like for example the number of blades in an impeller, are treated as real numbers in the optimization algorithm. They are converted back to integer values when the new geometries are created, based on the parameter vectors.

\subsubsection{Verification for test problems}

For verification purposes the implementation of the DE method has been applied to various test functions. The selected two-dimensional test functions are a parabolic test function $f_{1}$, one with multiple local minima $f_{2}$, i.e. Rastrigin's function, and function $f_{3}$, which has a large flat valley around the global minimum. All functions have a global minimum of $f=0$ at $\left(x_{1}, x_{2}\right)=(0,0)$. These functions are frequently used for verification of optimization algorithms (see for example $[33,57]$ ) and they are listed below

$$
\begin{gathered}
f_{1}\left(x_{1}, x_{2}\right)=x_{1}^{2}+x_{2}^{2} \\
f_{2}\left(x_{1}, x_{2}\right)=20+x_{1}^{2}+x_{2}^{2}-10\left[\cos \left(2 \pi x_{1}\right)+\cos \left(2 \pi x_{2}\right)\right] \\
f_{3}\left(x_{1}, x_{2}\right)=100\left(\left(x_{2}+1\right)-\left(x_{1}+1\right)^{2}\right)^{2}+\left(1-\left(x_{1}+1\right)\right)^{2}
\end{gathered}
$$

Since evolutionary methods are stochastic, multiple optimizations (100) have been carried out for each optimization problem. The parameters for the DE model are $T=0.5$, $C=0.5, P=20$ and $G=100$. The initial range for both $x_{1}$ and $x_{2}$ is set at $[-10,10]$, and the three test functions are sketched for this range in Fig. 4.1. The results are listed in table 4.1. From table 4.1 is deduced that for all test functions the global minimum is

Table 4.1: Test function optimization results. $T=0.5 ; C=0.5 ; P=20 ; G=100$.

\begin{tabular}{|c||c|c|c|c|c|c|}
\hline function & $x_{1 m}$ & $\sigma_{x_{1}}$ & $x_{2 m}$ & $\sigma_{x_{2}}$ & $f_{m}$ & $\sigma_{f}$ \\
\hline$f_{1}$ & $4.110^{-10}$ & $1.410^{-8}$ & $1.810^{-9}$ & $1.510^{-8}$ & $4.210^{-16}$ & $9.810^{-16}$ \\
\hline$f_{2}$ & $-6.110^{-8}$ & $3.710^{-7}$ & $-8.610^{-9}$ & $3.710^{-7}$ & $5.410^{-11}$ & $3.610^{-10}$ \\
\hline$f_{3}$ & $-2.010^{-2}$ & 0.19 & $-1.210^{-3}$ & 0.35 & $4.210^{-2}$ & 0.11 \\
\hline
\end{tabular}

found. For test function $f_{3}$ the standard deviations $\sigma_{x_{1}}$ and $\sigma_{x_{2}}$ are rather large, which 

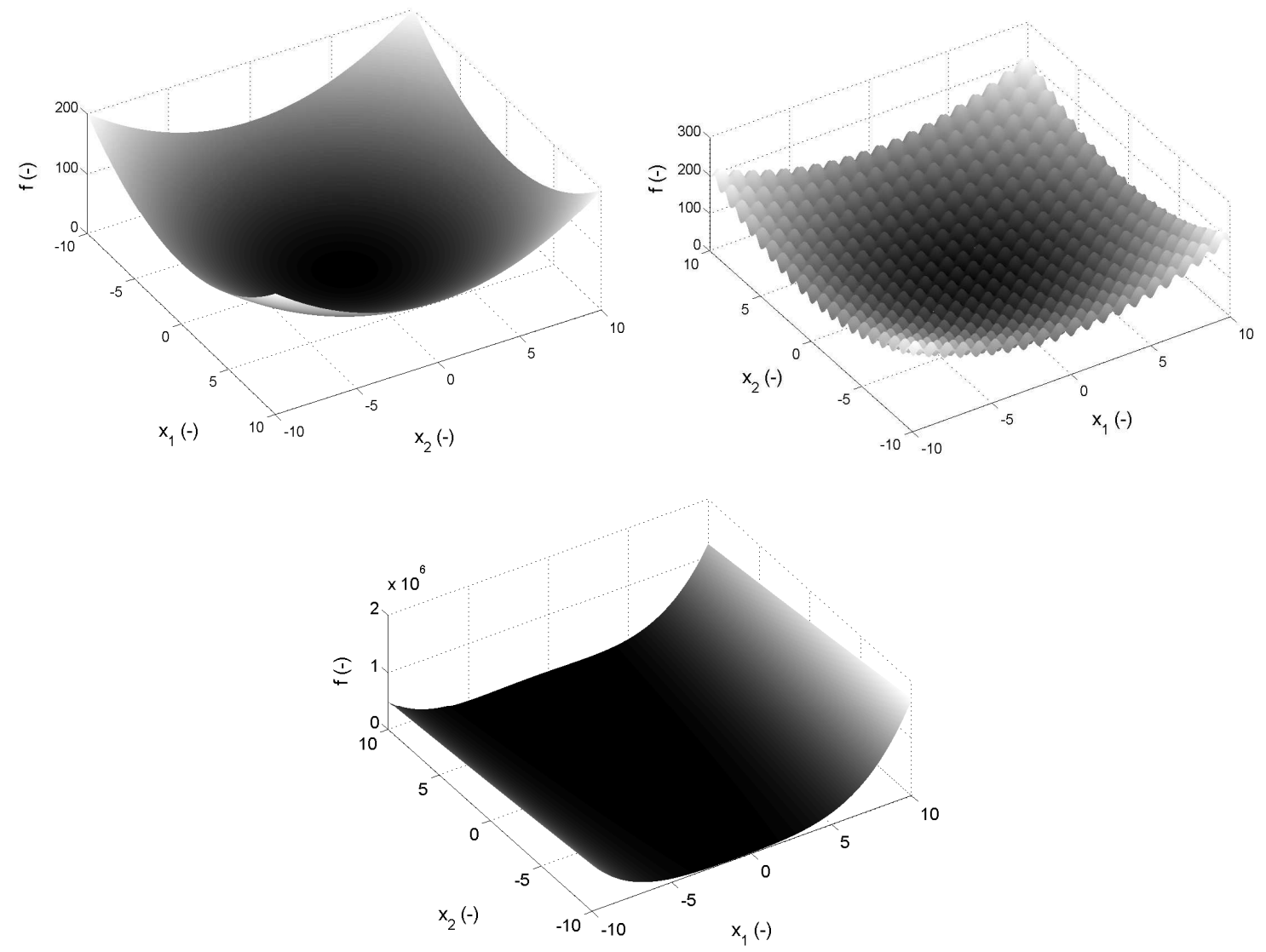

Figure 4.1: Test functions used for verification of the DE method. Functions shown are $f_{1}$ (top-left), $f_{2}$ (top-right) and $f_{3}$ (bottom).

is caused by the large flat valley around the optimum. The standard deviation in the function value $\sigma_{f}$ is also rather large, which is mainly caused by the much larger values of $f_{3}$ on the range of interest (see also Fig. 4.1). It is concluded that the implemented DE algorithm can be employed to solve the minimization problems, for complicated cost functions with many local minima (e.g. $f_{2}$ ). Additional tests have also been performed to show that Differential Evolution works well for non-differentiable cost functions.

\subsubsection{Applied numerical algorithm}

In the preceding sections the building blocks of the optimization method have been discussed. These are the parameterization, the cost function and the optimization algorithm. The employed numerical algorithm is shown in Fig. 4.2. The augmented potential flow package COMPASS is used to compute the flow field inside the impellers. This program has been developed at the University of Twente [46] and uses the Finite Element Method approach to solve the equations governing potential flow, as is described in Chapter 2. Note that a (non-zero) blade thickness distribution is incorporated in COMPASS. 


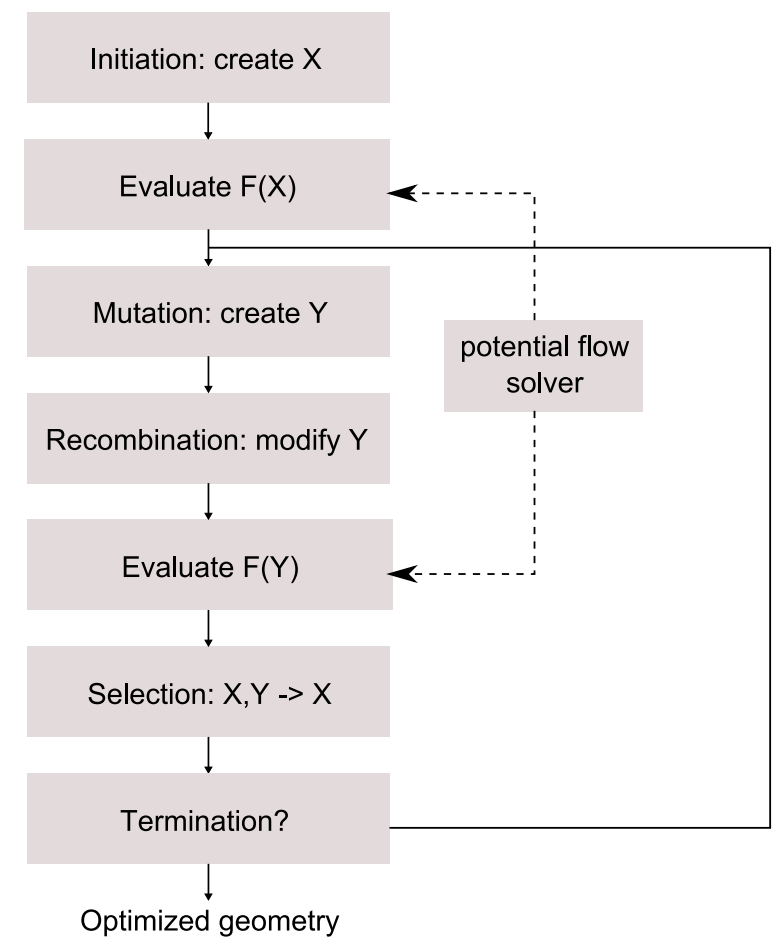

Figure 4.2: Optimization algorithm for impeller design.

\subsection{Optimization of a radial centrifugal pump im- peller}

In this section the optimization of a radial centrifugal pump impeller is presented. The optimization is based on an existing industrial radial impeller from Flowserve [25], which has been used previously for an optimization study [23]. Firstly, the original impeller is discussed. The parameterization of the impeller geometry and the definition of the cost function are treated subsequently. The optimization process and the obtained result are presented in section 4.3.4. Finally, in order to demonstrate the influence of the selected parameterization and cost function, additional optimizations have been carried out and the results of these optimizations are compared and discussed in section 4.3.5.

\subsubsection{Original impeller}

The original radial impeller is shown in Fig. 4.3 along with the blade angle distribution in the meridional plane. The dimensionless design parameters for the impeller are shown in table 4.2. The blades have zero stacking at the trailing edge, i.e. the blade is straight from hub to shroud at the trailing edge. A direct flow computation is carried out for the original impeller and as expected the computed inviscid-flow head coefficient $\psi_{i n v}$ is somewhat higher than the measured value, $\psi / \psi_{i n v}=0.88$. 
Table 4.2: Dimensionless characteristics of the original radial impeller at design point.

\begin{tabular}{|c|c|c|c|}
\hline$Z$ & $\phi$ & $\psi$ & $N_{s}$ \\
\hline 7 & 0.016 & 0.12 & 0.64 \\
\hline
\end{tabular}
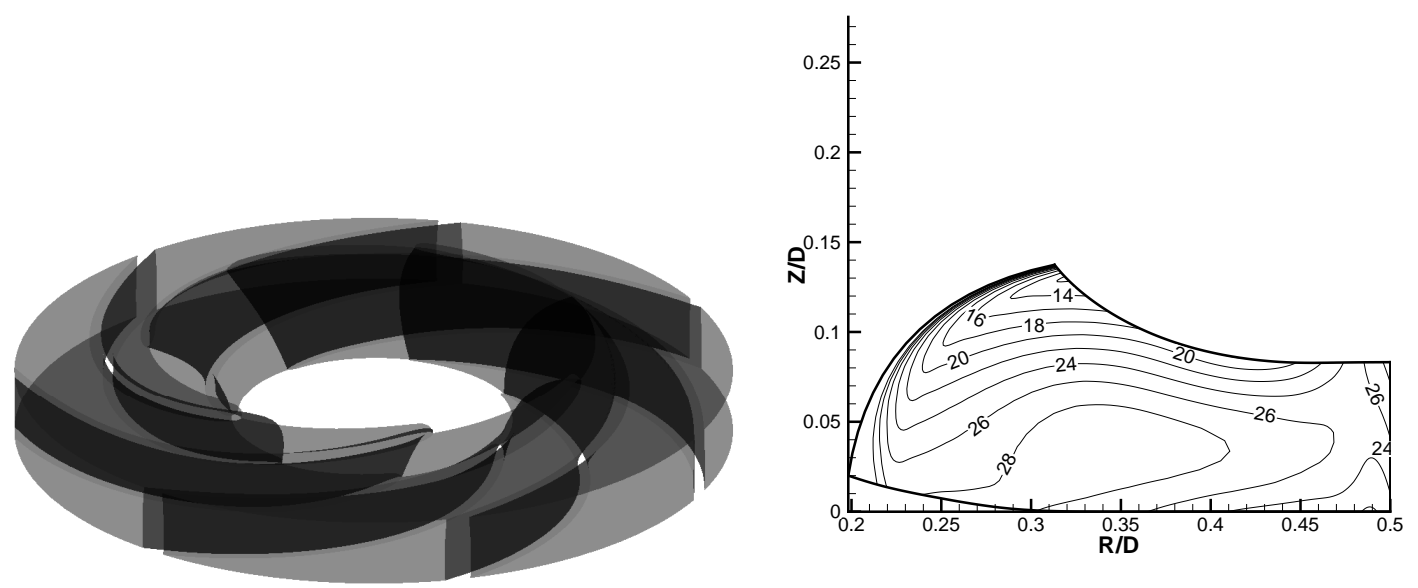

Figure 4.3: Original radial impeller: the impeller (left) and blade angle distribution $\beta(m, s)$ in the meridional plane (right).

\subsubsection{Parameterization}

The parameterization of the optimization determines the range of considered geometries and hence also the quality of the obtained optimum. Therefore, it has to be chosen adequately. In the present study the parameterization includes the number of blades, the blade curvature and the shroud curvature.

The first parameter employed is the number of blades of the impeller. For optical accessibility of the scale model, to be used in the validation experiments described in chapter 5 , the number of blades considered for optimization is reduced from 7 to a bounded range of $[4,6]$. This means that the original number of blades is not included in this range.

The second series of parameters is related to the blade angle distribution. The blade angle is taken relative to the circumferential direction, i.e. a small blade angle implies a more curved, and hence, a longer blade. The blade angle is specified at 10 different locations in the meridional plane, as is shown in Fig. 4.4. The blade angle at the trailing edge has been taken constant from hub to shroud. The bounded range for the blade angle is defined based on a deviation from the original blade angle $\beta_{i}^{0}$ at each location $i$. It is given by $\left[\beta_{i}^{0}-5^{\circ}, \beta_{i}^{0}+10^{\circ}\right]$. This approach is used to avoid a large number of invalid meshes in the optimization process.

The last two parameters, $\Delta z_{1}$ and $\Delta z_{2}$, are employed to parameterize the shroud curve in the meridional plane, at $r_{1} / D=0.34$ and $r_{2} / D=0.44$ respectively. The two parameters give an axial displacement in the meridional plane, as is shown in Fig. 4.4. It is given by 

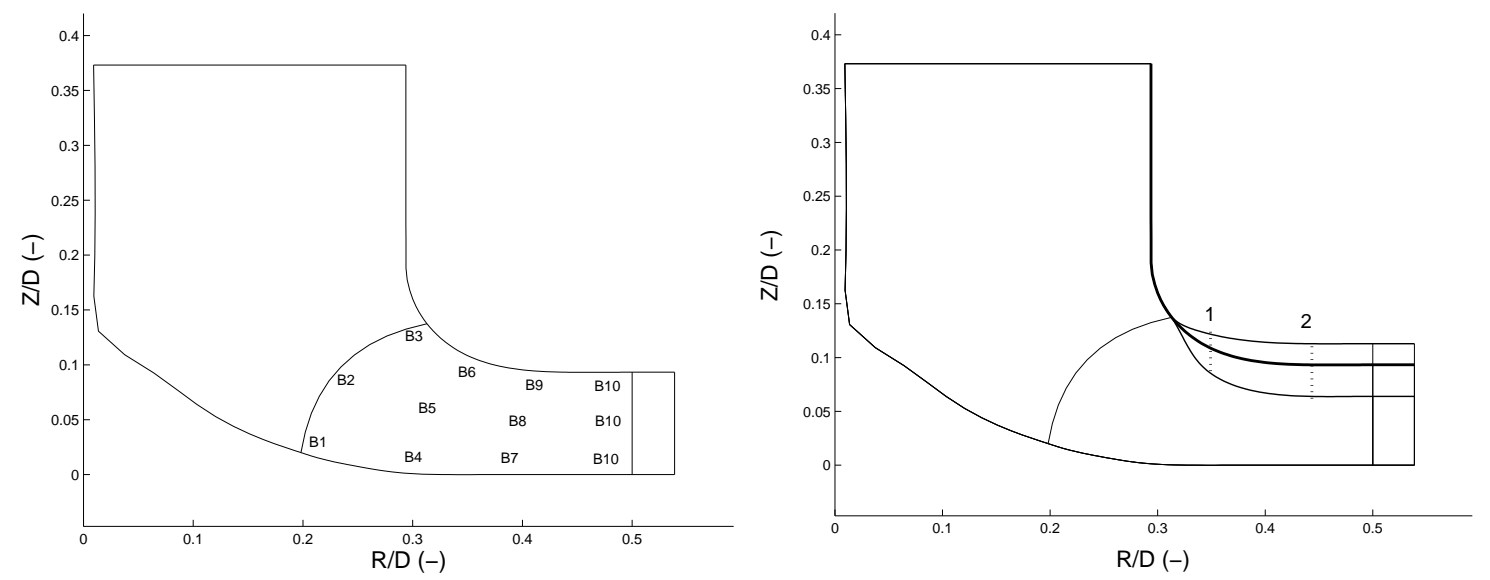

Figure 4.4: Meridional view of the impeller: parameterization of blade curvature (left) and parameterization of the meridional shroud curve (right).

$$
\begin{aligned}
& z_{1}=z_{1}^{0}+\Delta z_{1} \\
& z_{2}=z_{2}^{0}+\Delta z_{1}+\Delta z_{2}
\end{aligned}
$$

A cubic spline interpolation is used to describe the shroud curve from the leading edge up to point 2, after which the axial height remains constant. The bounded range for $\Delta z_{1}$ is $[-0.02 D, 0.03 D]$ and for $\Delta z_{2}$ it is $[-0.01 D, 0.01 D]$.

In summary, in total there are 13 parameters that describe geometrical variations, 1 for the number of blades, 10 for the blade angle distribution and 2 for the shroud shape. Note that in the inverse-design method described in the preceding chapter, the blade curvature is obtained as a result of the procedure. For the optimization method not only the blade curvature is considered, but other parameters can also be included easily. In this case these additional parameters describe the shroud curvature and the number of blades in the impeller. The optimization case discussed here is labeled $o p t_{4,6}$, referring to the selected bounded range for the number of blades.

\subsubsection{Cost function}

In this section the definition of the cost function for the current optimization is presented. For each geometry a flow computation is performed, for each flow rate considered, from which the selected objectives are evaluated. Firstly, the considered objectives are treated. Secondly, the definition of the lumped cost function is presented, which considers the performance of these objectives at different flow rates.

The first design objective that is considered is based on the inviscid-flow pump head $H$ generated by the impeller. The objective is to design a new impeller with a pump head coefficient $\psi=(g H) /\left(\Omega^{2} D^{2}\right)$ that is higher than or equal to the design target $\psi_{d}$. In this case the head coefficient of the original pump is taken as design target. The head 


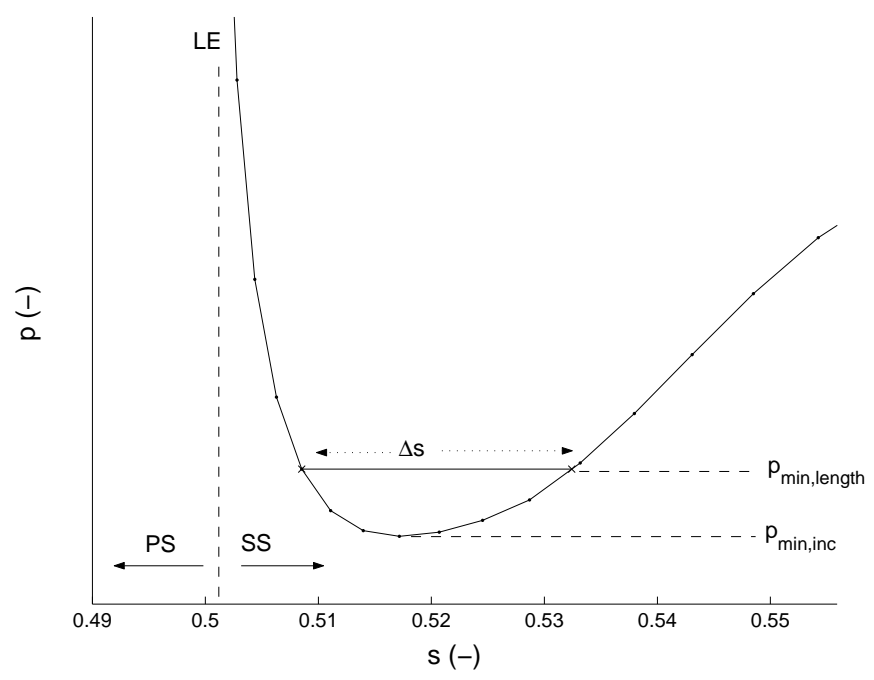

Figure 4.5: Pressure distribution around the minimum pressure point. The blade coordinate $s$ runs from the pressure side trailing edge $(s=0)$, via the leading edge $(s=0.5)$ to the suction side trailing edge $(s=1)$.

objective $f_{\psi}$ is therefore given by

$$
\begin{aligned}
f_{\psi}=1-\frac{\psi}{\psi_{d}} & \\
f_{\psi}=0 & \psi \geq \psi_{d}
\end{aligned}
$$

This formulation gives a penalty to designs with $\psi<\psi_{d}$.

The second design objective is that the boundary-layer losses are as low as possible. The boundary-layer losses $\Delta P_{\text {loss }}$ are estimated with the hub, shroud and blade surfaces using Eqn. (2.27). The loss objective $f_{\zeta}$ is determined by

$$
f_{\zeta}=\zeta=\frac{\Delta P_{l o s s}}{P_{H}}=\frac{\frac{1}{2} \int_{S} C_{D} \rho w^{3} d S}{\rho g Q H}
$$

where $P_{H}$ is the inviscid-flow hydraulic power which is defined in Eqn. (1.3) and $S$ the surface area of boundary walls, i.e. the hub, shroud and impeller blade surfaces. Note that the actual pump head $H$ of the geometry considered is utilized to compute the hydraulic power $P_{H}$, and not the design target $H_{d}$.

The third and fourth objectives are cavitation objectives. In Fig. 4.5 the pressure distribution around a pressure minimum is sketched as a function of the dimensionless blade coordinate $s$, running from the pressure side trailing edge $(s=0)$, via the leading edge $(s=0.5)$ to the suction side trailing edge $(s=1)$. Since both the value and the width of the suction peak are of importance, two cavitation criteria are employed. The first is the cavitation inception coefficient $\kappa_{i}$, which is based on the lowest pressure $p_{\text {min }, \text { inc }}$ on the impeller blade. This inception criterion is also discussed in chapter 1 . The second criterion is the cavitation width coefficient $\kappa_{w}$, which is based on the pressure $p_{\text {min,length }}$, at which the width of the pressure peak exceeds a specified dimensionless width $\Delta s$, see 
Fig. 4.5. In the current optimization $\Delta s=0.02$. The cavitation objectives $f_{\kappa i}$ and $f_{\kappa w}$ are defined by

$$
\begin{aligned}
f_{\kappa_{i}} & =\kappa_{i}=\frac{g \mathrm{NPSH}_{i}}{\Omega^{2} D^{2}} \\
f_{\kappa_{w}} & =\kappa_{w}=\frac{g \mathrm{NPSH}_{w}}{\Omega^{2} D^{2}}
\end{aligned}
$$

where the NPSH is computed based on $p_{\min , i n c}$ for the inception objective $f_{\kappa_{i}}$ and based on $p_{\text {min,length }}$ for the cavitation width objective $f_{\kappa_{w}}$ (see Eqn. (1.5) for a definition of NPSH).

At a given flow rate $Q_{j}$ the cost function $F_{j}$ is determined by summing over the objectives $i$ using the weight factors $c_{j, i}$

$$
F_{j}=B_{j}\left(c_{j, \psi} f_{j, \psi}+c_{j, \kappa_{i}} f_{j, \kappa_{i}}+c_{j, \kappa_{w}} f_{j, \kappa_{w}}+c_{j, \zeta} f_{j, \zeta}\right)
$$

where $B$ is the back-flow factor, which is equal to 1 if back-flow does not occur and equal to 4 if it does occur. This approach is utilized to penalize impellers with back flow. The cost function, formulated in Eqn. (4.10), is evaluated at three different flow rates, i.e. at $80 \%, 100 \%$ and $120 \%$ of the design flow rate $Q_{d}$. In this way the performance of the impeller is optimized for a range of flow rates, rather than for a single design flow rate.

The total cost function is determined by summing over the flow rates $j$

$$
F=\sum_{j=1}^{3} F_{j}
$$

The weight factors $c_{j, i}$ for the $j$-th flow rate are chosen such that the contribution of each objective to the cost function is of similar order of magnitude, see table 4.3. This was achieved by investigating the variation of the objectives for several geometries, before the actual optimization was carried out. The head has only been considered at the design

Table 4.3: Weight factors for the optimization.

\begin{tabular}{|c|c|c|c|c|}
\hline$Q / Q_{d}$ & $c_{\psi}$ & $c_{\zeta}$ & $c_{\kappa_{i}}$ & $c_{\kappa_{w}}$ \\
\hline 0.80 & 0 & 2.5 & 0.5 & 7.5 \\
\hline 1.00 & 10.0 & 10.0 & 2.0 & 30.0 \\
\hline 1.20 & 0 & 2.5 & 0.5 & 7.5 \\
\hline
\end{tabular}

flow rate, while the importance of the other objectives is selected to be high for the design flow rate and low for the off-design flow rates.

\subsubsection{Optimization results}

The convergence rate of the optimization process is shown in Fig. 4.6. For $30 \%$ of all considered geometries a valid mesh could not be obtained. These geometries are given a high value for the cost function $\left(10^{30}\right)$ in order to remove them from consideration. The optimized impeller has a cost function of 1.35, compared to the value of 1.68 of the original impeller. 


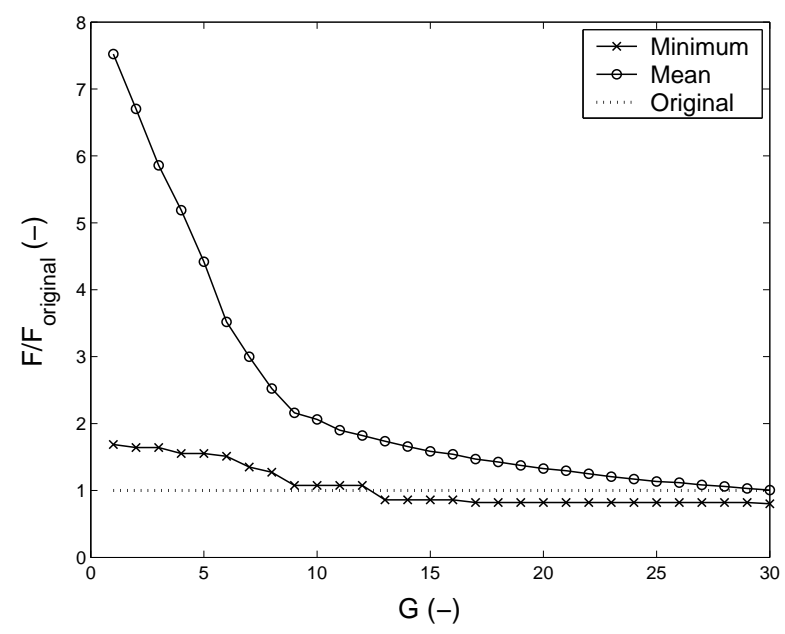

Figure 4.6: Convergence history of optimization case $o p t_{46}$. The cost function $\left(F / F_{\text {original }}\right)$ of the population mean, minimum and original geometry plotted as a function of the number of generations $G$.

The optimized impeller has 6 blades, which is the maximum number of blades allowed in the present optimization (but one less than that of the original impeller). The original and optimized blade shapes are compared in Fig. 4.7. The optimized blade shape is more curved than the original blade shape (smaller blade angles), except at the trailing edge in order to meet the head requirement. The optimized shroud curve differs slightly from the original one, see Fig. 4.8. The optimized impeller is shown in Fig. 4.8 on the right-hand side, where the shroud is not displayed for clarity.

Table 4.4 shows the contributions to the cost function at the three flow rates considered for the optimized and for the original impeller. The cavitation characteristics of the impeller, given by the objectives $f_{\kappa_{i}}$ and $f_{\kappa_{w}}$, have been improved, whereas the loss objective $f_{\zeta}$ and head objective $f_{\psi}$ are comparable to those of the original impeller. If a larger number of blades than 6 had been allowed, the loss objective could have been reduced further, as will be shown in section 4.3.5. The head objective $f_{j, \psi}$ is only considered at the design flow rate (for off design conditions $c_{j, \psi}=0$ ) and therefore both the original and optimized design meet the head requirement and the difference observed in $f_{\psi}$ for the high flow rate has no influence.

For the flow rates considered back-flow does not occur, for either the original or the optimized impeller. The distributions of the relative velocity at the design flow rate $Q_{d}$ are plotted in Fig. 4.9 as a function of the meridional distance $m$ for the original and optimized impeller. The peak in the velocity distribution near the leading edge is clearly reduced for the optimized design. Furthermore, the velocity difference across the blades is somewhat higher for the optimized design. This is caused by the fact that the optimized design has 6 blades, whereas the original has 7 . The difference is not very large though, since the optimized blade is longer than the original.

In Fig. 4.10 the corresponding static pressure distributions are plotted. The pressure peak at the suction side, near the leading edge, is not as deep for the optimized geometry compared to that for the original geometry, indicating that from the point of view of 

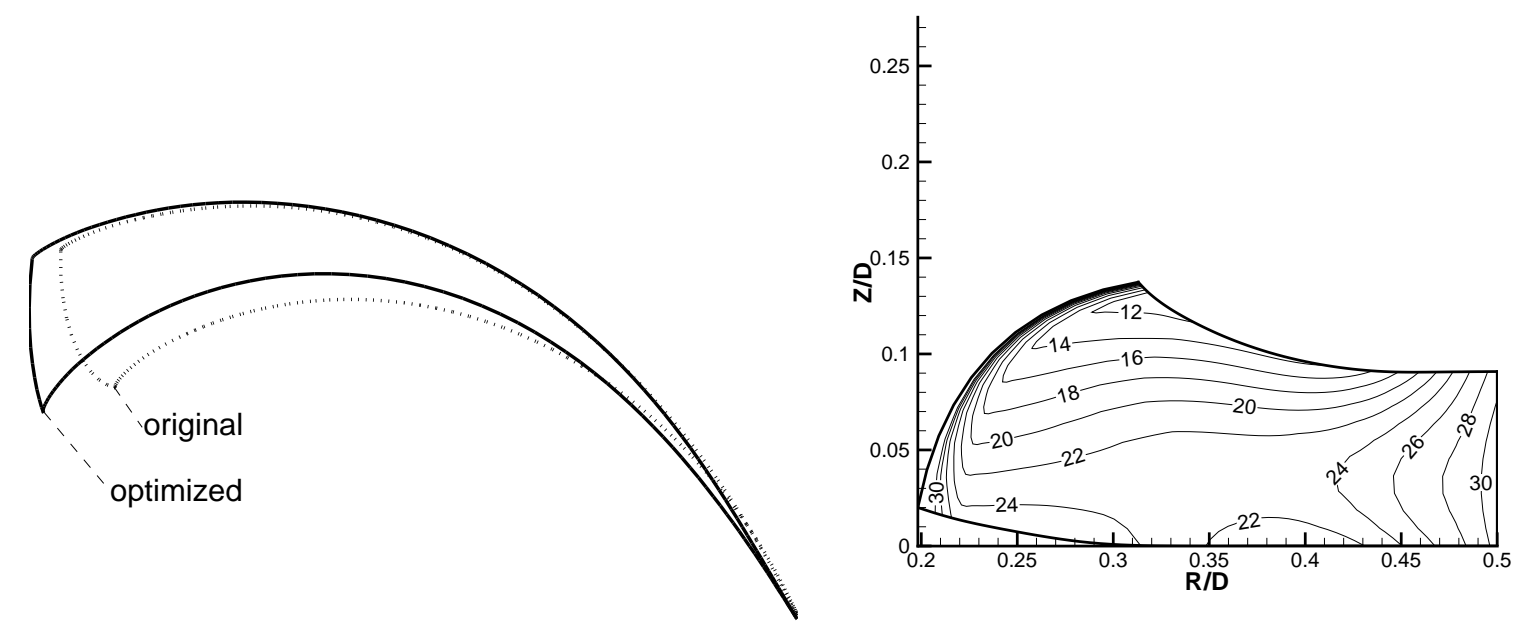

Figure 4.7: Top view of the blade contours of the original and optimized blade shape (left) and the optimized blade angle distribution (right).
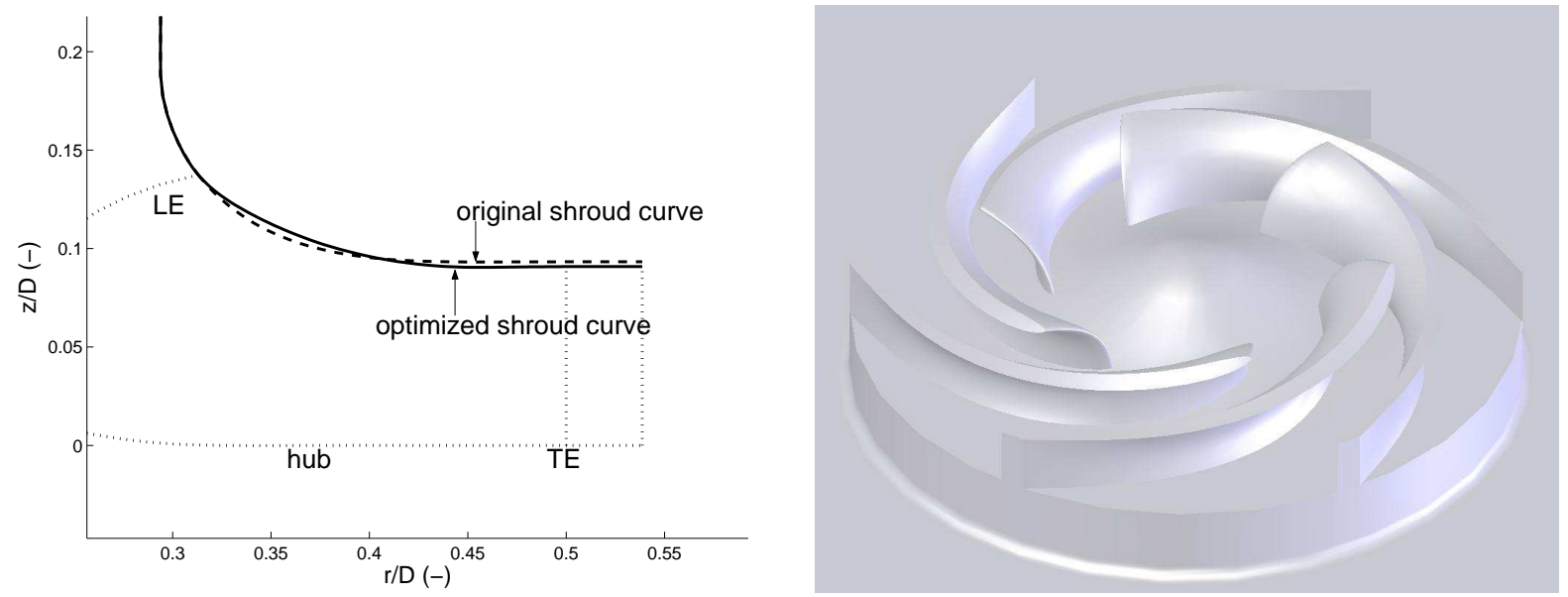

Figure 4.8: Optimized and original meridional shroud curve (left) and the optimized impeller (right). 
Table 4.4: Comparison of objectives for the optimized and original impeller.

\begin{tabular}{|c||c|c|c|c|}
\hline \multicolumn{1}{|c||}{$Q / Q_{d}$} & $f_{j, \psi}$ & $f_{j, \zeta}$ & $f_{j, \kappa_{i}}$ & $f_{j, \kappa_{w}}$ \\
\hline \hline \multicolumn{1}{|c||}{$j$} & \multicolumn{5}{c|}{ Optimized Impeller } \\
\hline 0.80 & 0.000 & 0.035 & 0.082 & 0.019 \\
\hline 1.00 & 0.000 & 0.039 & 0.045 & 0.012 \\
\hline 1.20 & 0.073 & 0.046 & 0.098 & 0.010 \\
\hline \hline \multicolumn{1}{|c||c|c|c|c|c|c|c|}{ Original Impeller } \\
\hline 0.80 & 0.000 & 0.035 & 0.226 & 0.017 \\
\hline 1.00 & 0.000 & 0.038 & 0.133 & 0.015 \\
\hline 1.20 & 0.100 & 0.046 & 0.070 & 0.012 \\
\hline
\end{tabular}
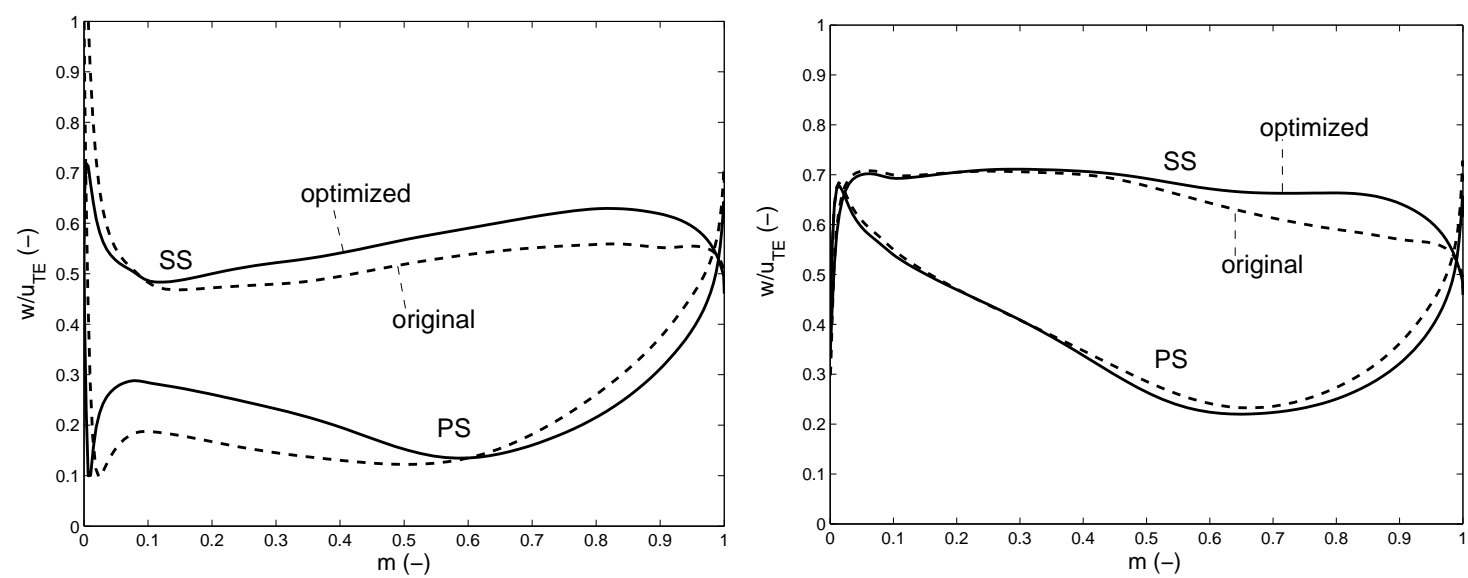

Figure 4.9: Relative velocity distributions along the blade-hub intersection (left) and the bladeshroud intersection (right) at design condition. PS indicates the pressure side, SS the suction side.

cavitation the flow conditions near the leading edge are more favorable for the optimized design than for the original impeller.

It is interesting to see whether the four objectives that are used to evaluate the performance of a geometry are correlated or not. To this end the cavitation inception coefficient $\kappa_{i}$, the pump head coefficient $\psi$ and the loss coefficient $\zeta$ are compared in Fig. 4.11 for all geometries considered in the optimization (the cavitation width objective is not considered, since it is complementary to the cavitation inception criterion). The pump head coefficient $\psi$ and the loss coefficient $\zeta$ are negatively correlated. The corresponding objectives $f_{\psi}$ and $f_{\zeta}$ are positively correlated, since high head coefficients and low loss coefficient are beneficial for the cost function (see section 4.3.3). The explanation for these observations is that blades with a higher pump head are less curved (near the trailing edge) and therefore have a smaller surface area $S$ and hence smaller losses, as can be seen from the definition of the loss objective in Eqn. (4.7). Furthermore, this loss objective $f_{\zeta}$ is defined as the ratio of power loss and the hydraulic power, which scales with the pump head. Therefore, higher pump heads lead to lower loss coefficients. The cavitation inception coefficient $\kappa_{i}$ and the head coefficient $\psi$ are practically uncorrelated, as are their 

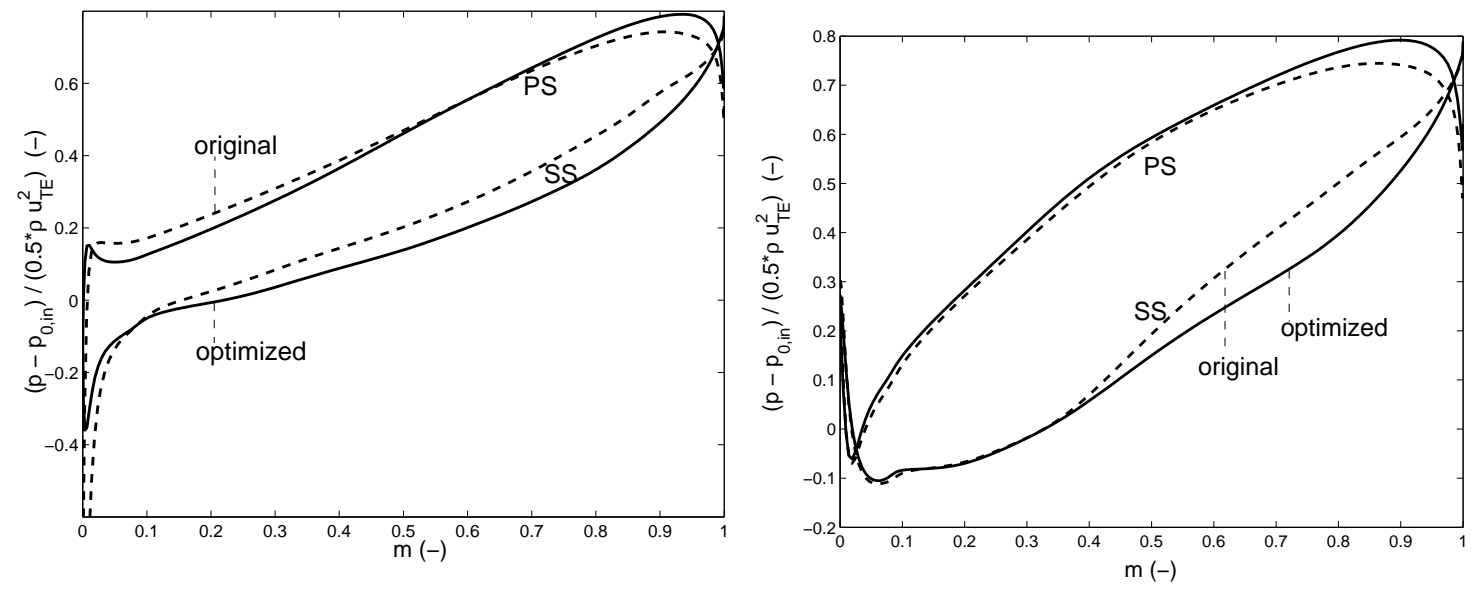

Figure 4.10: Static pressure distribution along the blade-hub intersection (left) and the bladeshroud intersection (right) at design condition. PS indicates the pressure side, SS the suction side.

corresponding objectives $f_{\kappa_{i}}$ and $f_{\psi}$. This can be seen in Fig. 4.11 on the right hand side. The explanation is that the shape of the blade near the leading edge mainly determines the cavitation characteristics, whereas the part near the trailing edge mainly determines the head coefficient. Thus $\kappa_{i}$ and $\psi$ are predominantly determined by different parts of the blade.
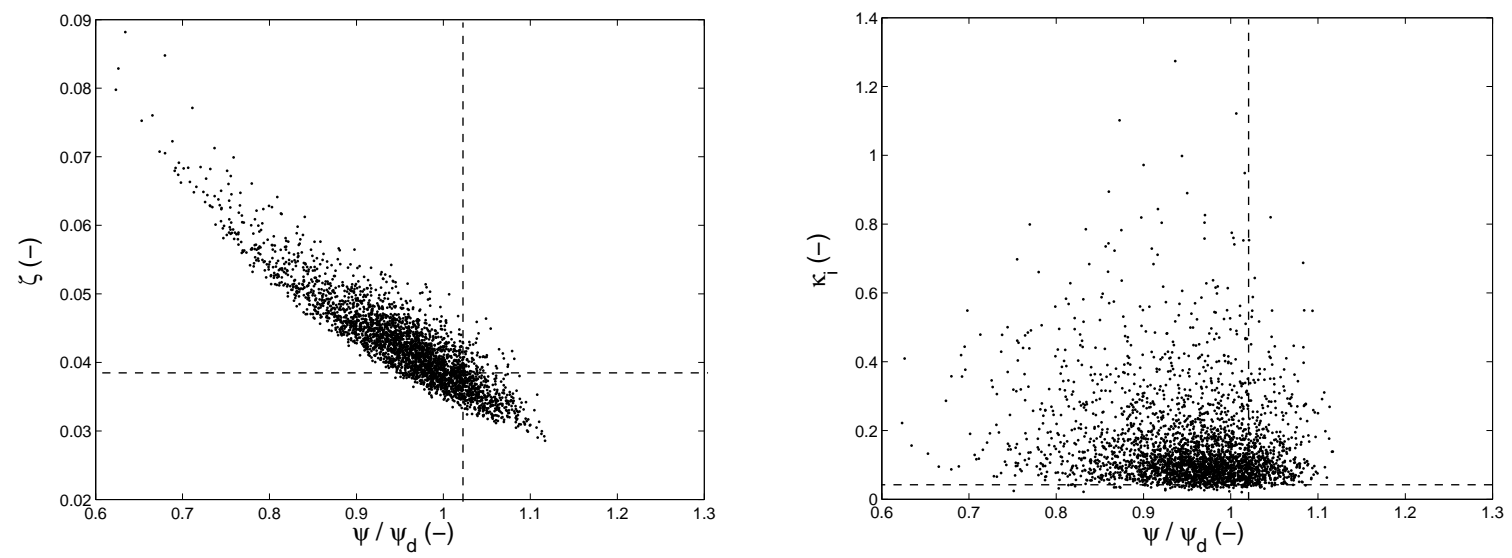

Figure 4.11: Loss coefficient $\zeta$ (left) and cavitation inception coefficient $\kappa_{i}$ (right) as function of the head coefficient $\psi / \psi_{d}$ for all geometries considered in the optimization at the design flow rate $\phi_{d}$. The dashed lines indicate the objectives for the optimum.

In appendix A a parameter sensitivity analysis is carried out. The purpose of this analysis is to investigate which parameters have a large influence on the cost function and to determine whether the optimized geometry indeed is the optimum for the current parameterization and formulation of the cost function. It is concluded that for the present parameterization and formulation of the cost function, the optimized design is indeed optimal. 


\subsubsection{Additional optimizations}

In this section the influence of parameterization and formulation of the cost function on the resulting design is investigated. For the parameterization the focus will be on the bounded range for the number of blades. For the cost function a slightly different definition of the head objective is considered. This results in a number of additional optimizations which have been carried out. Firstly, the bounded rage for the number of blades is considered.

The original impeller has 7 blades whereas the first optimization is carried out for a bounded range of $[4,6]$, motivated by the requirement to have a good optical accessibility of the scale model to be used for experiments. This first optimization is labeled $o p t_{4,6}$, where 4,6 denotes the bounded range for the optimization. Two further optimizations are presented in this section for a fixed number of 7 blades, $o p t_{7}$, and for a bounded range of $[4,10], o p t_{4,10}$. These optimizations are summarized in the top half of table 4.5. The cost function is determined in the same way as described in section 4.3.3.

The definition of the cost function also can have a large influence on the resulting optimized geometry. Therefore an extra optimization is carried out in which the head objective $f_{\psi}$ is computed in a slightly different manner. Instead of having an objective in which the head coefficient $\psi$ needs to exceed a certain design target $\psi_{d}$, the new objective is to obtain a head coefficient $\psi$, which is as close as possible to $\psi_{d}$. This alternative head objective $f_{\psi, 2}$ is defined by

$$
f_{\psi, 2}=\sqrt{\frac{\left(\psi-\psi_{d}\right)^{2}}{\psi_{d}^{2}}}=\left|1-\frac{\psi}{\psi_{d}}\right|
$$

In Fig. 4.12 a sketch is given of the original and the alternative head objective. It can

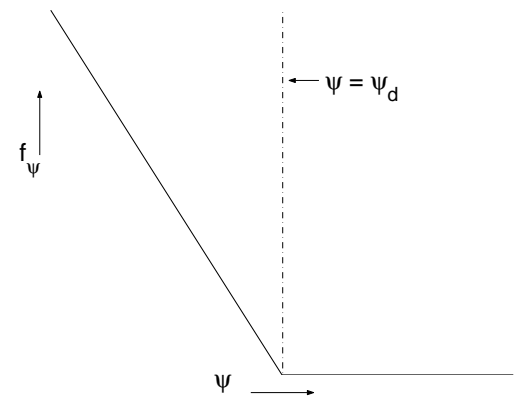

(a) original head objective

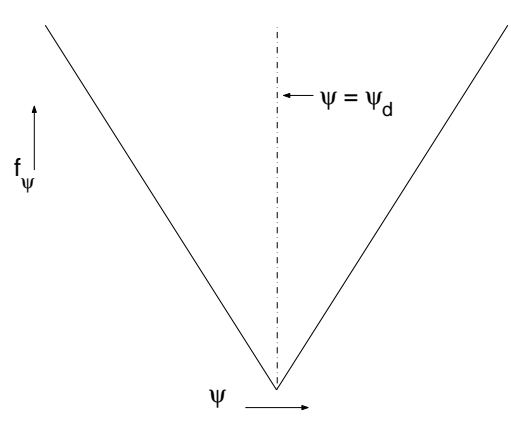

(b) alternative head objective

Figure 4.12: Original and alternative head objectives.

be argued that $f_{\psi, 2}$ is a more suitable approach to define the head objective, since the meridional geometry is often chosen based on the specific speed of an impeller, which also includes the pump head. The cost function which uses the alternative definition of the head objective $f_{\psi, 2}$ is denoted as $F_{2}$. The conventional cost function is referred to as $F_{1}$.

The optimization based on cost function $F_{2}$ is denoted as opt $t_{f 2}$ (see table 4.5). The purpose of this optimization is twofold. First of all, the goal is to investigate the consequences of using a different formulation of the cost function, or more specifically the head 
objective. Secondly, the purpose is to study how much the cost function can be further reduced, when continuing the optimization process for a larger number of generations $G$.

The parameters for the additional optimizations $o p t_{7}, o p t_{4,10}$ and $o p t_{f 2}$ are summarized in table 4.5. The optimization results are discussed in the next sections.

Table 4.5: Summary of all optimization cases.

\begin{tabular}{|c|c|c|c|}
\hline optimization & blade number range & cost function & $G$ \\
\hline opt $_{4,6}$ & {$[4,6]$} & $F_{1}$ & 30 \\
\hline$o p t_{7}$ & {$[7]$} & $F_{1}$ & 30 \\
\hline opt $_{4,10}$ & {$[4,10]$} & $F_{1}$ & 30 \\
\hline opt $_{f 2}$ & {$[4,10]$} & $F_{2}$ & 90 \\
\hline
\end{tabular}

\section{$o p t_{7}$}

For this optimization the number of blades is fixed at 7 , and the default cost function $F_{1}$ is employed. This will give a fair comparison between the original impeller, which has 7 blades, and an optimized impeller. The results for this optimization are listed in table 4.6. The cost function $F_{1}$ has been reduced significantly to $F_{1}=1.20$, compared to the cost function of the original $\left(F_{1}=1.68\right)$ and the first optimized geometry $\left(F_{1}=\right.$ 1.35). The further improvement is mainly in the loss objective $f_{\zeta}$ and the cavitation inception objective $f_{\kappa_{i}}$, see table 4.6. The optimized meridional shroud curve is shown in Fig. 4.14 and the blade angle distribution at the hub is shown in Fig. 4.15. Note that the meridional shroud curve for this design has a shape which may be undesirable for construction purposes. If this is indeed the case a different bounded range has to be selected for the parameters $\Delta z_{1}$ and $\Delta z_{2}$, which describe the shroud curvature in the meridional plane. When compared to the original design, the width of the impeller has clearly increased and the blade angle at the hub is also slightly higher, leading to an increased head coefficient of $\psi=1.05 \psi_{d}$. The increase in impeller width also leads to a reduction in relative velocity, thus leading to a lower loss coefficient $\zeta$.

opt $_{4,10}$

In this optimization the bounded range for the number of blades is set at $[4,10]$. Furthermore, the default cost function $F_{1}$ is utilized. This optimization is performed in order to allow for impellers with a large number of blades. The cost function $F_{1}$ of the optimum is reduced from $F_{1}=1.68$ for the original geometry to $F_{1}=1.07$ for the optimized geometry, which is also significantly lower than the cost function $F_{1}$ obtained for the other considered optimizations. The main improvement is in the loss objective, which has been reduced by a substantial margin. The optimized impeller has 10 blades and the width of the impeller is increased, see Fig. 4.14. The blade angle distribution at the hub is shown in Fig. 4.15.

The increase in impeller width, the large blade angle at the trailing edge and the large number of impeller blades, all lead to a large increase in the pump head coefficient, $\psi=1.18 \psi_{d}$. This large increase in head coefficient leads to a significant reduction in the loss objective, which is seen from the definition of the loss objective in Eqn. (4.7). 
Furthermore, for the determination of the loss coefficient $\zeta$ it is assumed that the boundary layers are thin relative to the channel width and that their influence on the core flow can be neglected. However, when the number of blades is increased, this assumption may no longer be justified. This effect has not been considered in the current optimizations.

Although this optimization shows a significant improvement of the objectives, most of the improvements are attributed to the increase in the pump head coefficient $\psi$. In the next optimization the alternative definition of the pump head objective $f_{\psi, 2}$ is utilized to investigate its influence.

$o p t_{f 2}$

The purpose of this optimization is twofold. We consider the influence of the definition of the cost function and the number of generations used in the optimizations. The optimization is carried out for cost function $F_{2}$, which uses the alternative definition of the head objective $f_{\psi, 2}$, see Eqn. (4.12), and it is run for 90 generations, instead of the usual 30 generations.

Firstly, it is investigated what the influence of the number of generations is on the obtained optimum. Fig. 4.13 shows the convergence plot of the cost function for this optimization. After 30 generations the cost function for the optimum is $F_{2}=1.29$ and after 90 generations it has been further reduced to $F_{2}=1.21$. When compared to the cost function for the original geometry $\left(F_{2}=1.68\right)$, this means an improvement of $24 \%$ after 30 generations and an improvement of $28 \%$ after 90 generations. Therefore, it is concluded that 30 generations, employed for most optimizations in this chapter, is sufficient to obtain a substantial improvement in performance.

Next, the focus will be on the optimized geometry. When compared to the original geometry, the improvement of the optimized geometry is both in the cavitation objectives and the boundary layer losses. As expected by the formulation of the head objective, the head coefficient for the optimized geometry is close to the design target, $\psi=0.99 \psi_{d}$. The optimized impeller has 8 blades, and the optimized shroud curve and blade angle distribution at the hub are shown in Fig. 4.14 and 4.15, respectively. Since the impeller has more blades than the original impeller geometry, as well as a slightly higher blade angle at the trailing edge, the impeller width is reduced in order to obtain a similar head as for the original impeller. 


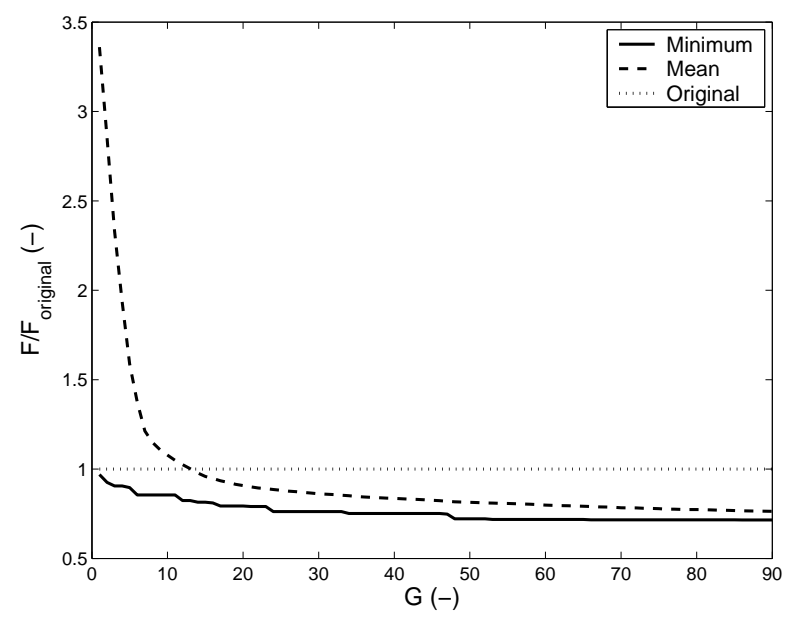

Figure 4.13: Convergence history of optimization case $o p t_{f 2}$. The cost function $\left(F / F_{\text {original }}\right)$ of the population mean, minimum and original geometry plotted as a function of the number of generations $G$.

\section{Discussion of additional optimizations}

The additional optimizations have shown the influence of both the selected range for the number of blades and the formulation of the cost function, in this case the head objective. The meridional shroud curve and the blade angle distribution at the hub are compared for all optima and the original geometry in Fig. 4.14 and 4.15, respectively. The optimized geometries all have a similar blade angle at the hub near the leading edge, which results in favorable cavitation characteristics. The impeller optimized for a blade range of $[4,6]$, $o p t_{4,6}$, has the largest blade angle at the trailing edge to compensate for the relative low number of blades (see table 4.6). A low number of blades corresponds to a large slip factor and hence a relative low pump head.

The build up of the cost functions for the various designs is summarized in table 4.6, where $f_{\psi}$ indicates the original head objective, used in cost function $F_{1}$ and $f_{\psi, 2}$ indicates the modified head objective, employed in the calculation of cost function $F_{2}$.

From this table it is concluded that for cost function $F_{1}$, the optimization case $o p t_{4,10}$, with 10 impeller blades, results in the best overall optimum. However, the large head coefficient for this design results in a large value for cost function $F_{2}$. The best geometry for cost function $F_{2}$ is $o p t_{f 2}$, which has 8 impeller blades. This is hardly surprising, since this is the only optimization using $F_{2}$ as optimization cost function. Since the head coefficient of the optimum of $o p t_{4,10}$ is very large, its corresponding meridional geometry might have to be altered. Furthermore, especially in the case of a large number of blades it might no longer be justified to neglect the influence of the boundary layers on the core flow.

The original optimized impeller opt $_{4,6}$ has been manufactured in PMMA for use in experimental investigations. This optimized impeller was selected for its good optical accessibility due to the relatively low number of blades. The PIV measurements carried out with this impeller are presented in chapter 5 . 


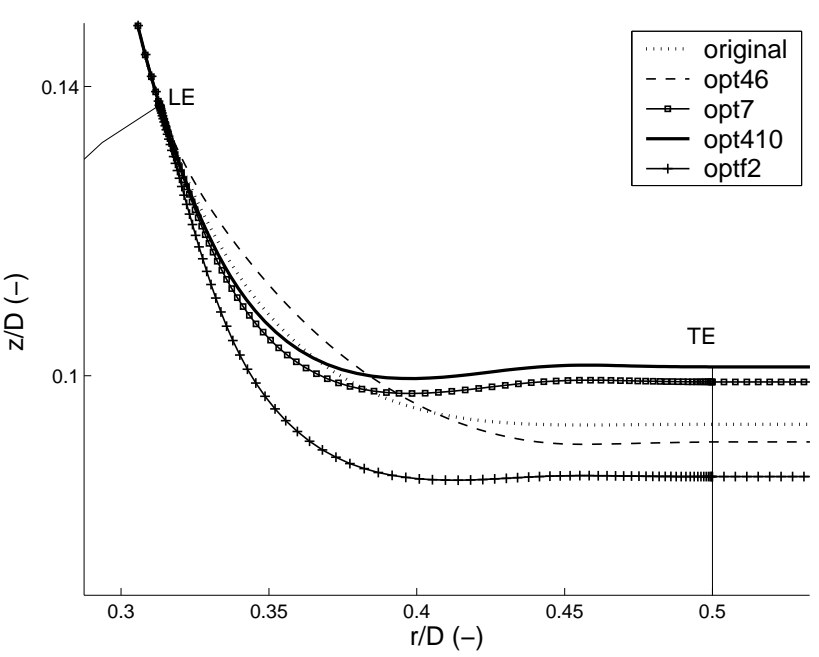

Figure 4.14: Optimized and original meridional shroud curves. Note that the scaling for the $r$-axis and $z$-axis is different (z-axis is stretched).

Table 4.6: Optimization objectives summed over the considered flow rates $j$.

\begin{tabular}{|l|c|c|c|c|c|}
\hline objective & original & $o p t_{4,6}$ & $o p t_{7}$ & $o p t_{4,10}$ & $o p t_{f 2}$ \\
\hline$\Sigma c_{\psi} f_{\psi}$ & 0.000 & 0.000 & 0.000 & 0.000 & 0.011 \\
\hline$\Sigma c_{\psi} f_{\psi, 2}$ & 0.000 & 0.213 & 0.492 & 1.838 & 0.011 \\
\hline$\Sigma c_{\zeta} f_{\zeta}$ & 0.582 & 0.591 & 0.544 & 0.411 & 0.572 \\
\hline$\Sigma c_{\kappa_{i}} f_{\kappa_{i}}$ & 0.414 & 0.180 & 0.099 & 0.091 & 0.088 \\
\hline$\sum c_{\kappa_{w}} f_{\kappa_{w}}$ & 0.687 & 0.583 & 0.560 & 0.570 & 0.539 \\
\hline \hline$F_{1}$ & 1.683 & 1.354 & 1.202 & 1.073 & 1.210 \\
\hline$F_{2}$ & 1.683 & 1.566 & 1.694 & 2.911 & 1.210 \\
\hline \hline$Z$ & 7 & 6 & 7 & 10 & 8 \\
\hline
\end{tabular}

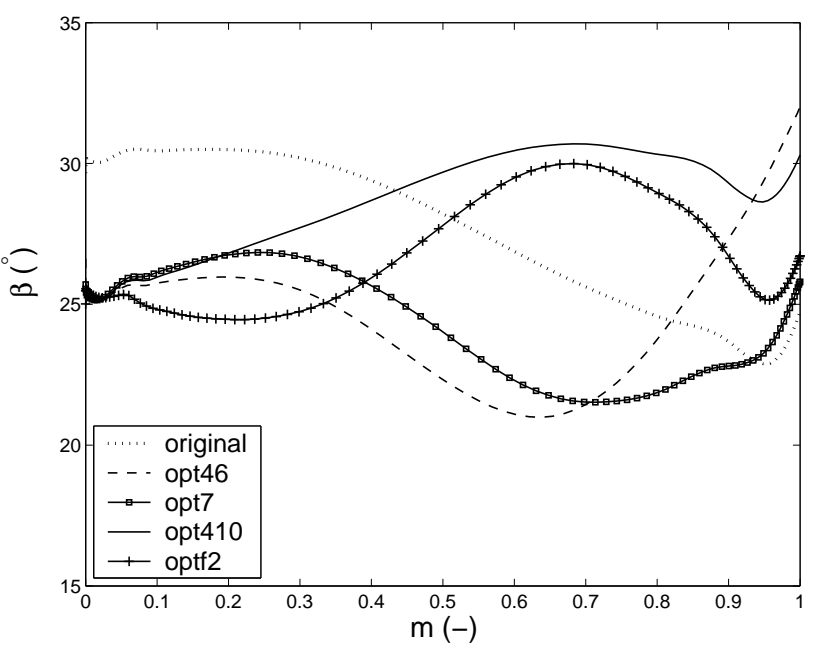

Figure 4.15: Optimized and original blade angle distributions at the hub. 


\subsection{Inverse-optimization of a mixed flow impeller}

The developed optimization method presented in this chapter, based on Differential Evolution, can be employed for different optimizations as well. This is accomplished by employing an alternative method for obtaining the flow solution instead of the direct method utilized (see flowchart in Fig. 4.2). In the preceding chapter an inverse-design method has been presented. This inverse-design method will now be used in the optimization method. This hybrid approach is labeled inverse-optimization. Such inverse-optimizations have also been carried out by Peng et al. [51].

Here an inverse-optimization is presented of the same mixed flow impeller which has been treated in section 3.6 of chapter 3. The parameterization, the cost function and the optimization results will be discussed next.

\subsubsection{Parameterization}

In the preceding chapter it has been shown how the mean-swirl distribution can be modified in order to improve impeller performance. Here the mean-swirl distribution is parameterized. Since the impeller geometry is determined from the mean-swirl distribution, its parameterization yields an indirect parameterization of the impeller geometry.

The parameterization of the mean-swirl distribution is based on a cubic spline representation of the distribution at the hub-blade and at the shroud-blade intersection. A cubic spline is constructed based on 3 points $\left(m_{i}, f\left(m_{i}\right)\right)$, where $m$ is the dimensionless meridional coordinate from leading to trailing edge and $f_{i}(m)$ is the value of the dimensionless mean-swirl distribution at $m=m_{i}$. Two fixed points are located at the leading and trailing edge, i.e. $(0,0)$ and $(1,1)$, respectively. The third point is given by $\left(0.5, f_{2}(0.5)\right)$ where $f_{2}(0.5)$ is the value to be found in the optimization. This leads to two parameters for the geometry used in the optimization process, $f_{h u b}$, for the mean-swirl distribution along the hub-blade intersection, and $f_{s h r}$, for the mean-swirl distribution along the shroud-blade intersection. This parameterization is sketched in Fig. 4.16. The bounded ranges for the parameters $f_{h u b}$ and $f_{s h r}$ is $[0.3,0.7]$.

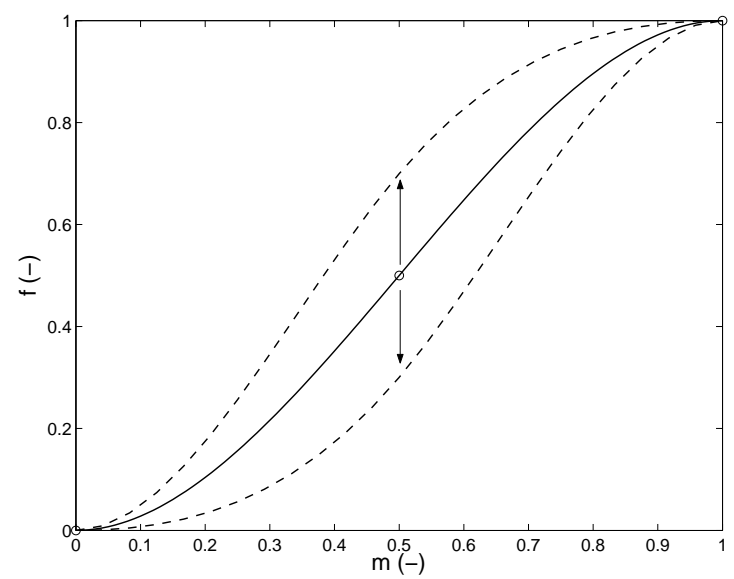

Figure 4.16: Parameterization of the dimensionless mean-swirl distribution $f$. 
The number of blades and the meridional geometry can also be parameterized, but this has not been considered in the present optimization.

\subsubsection{Cost function}

For the inverse-optimization the cost function is determined in a slightly different way, in compared with the direct optimization.

For the direct optimization the cost function has been computed at several flow rates. Since an inverse-design method uses a design flow rate as input to obtain a desired blade shape, no off-design considerations are taken into account, since this would lead to different blade shapes. Moreover, the design head $H_{d}$ is used as input for the inverse design method. Therefore, it does not need to be included in the cost function for an inverse-optimization.

For the cavitation characteristics we only consider the cavitation inception objective $f_{\kappa_{i}}$ in this investigation. Furthermore, the boundary layer loss objective $f_{\zeta}$ is also taken into account. Moreover, as was the case for the direct optimization, geometries with back-flow are penalized by a penalty factor $B$. For geometries without back-flow $B=1$ and for geometries with back-flow $B=4$.

In the preceding chapter geometries are analyzed utilizing the velocity loading $\chi$ on the blades. Therefore, a new velocity loading objective $f_{\chi}$ is added for the inverse-optimization

$$
f_{\chi}=\chi_{\max }=\max \frac{w^{s s}-w^{p s}}{\bar{w}}
$$

Summarizing, the cost function is evaluated at the design flow rate and is given by

$$
F=B\left(c_{\kappa_{i}} f_{\kappa_{i}}+c_{\zeta} f_{\zeta}+c_{\chi} f_{\chi}\right)
$$

The weight factors are chosen such that approximately all objectives have an equal importance in the cost function, i.e. $c_{\kappa}=80, c_{\zeta}=20$ and $c_{\chi}=1$. This has been achieved by considering several inverse-design computations for different sets of parameters.

\subsubsection{Optimization result}

Since the number of parameters for this optimization is rather small, it is sufficient to consider a relative small population size, $P=20$. Furthermore the number of generations has been selected at $G=20$, leading to a total of 400 inverse-design computations. Note that each inverse-design computation requires many flow solutions. The model parameters for the DE method are selected at $T=0.5$ and $C=0.8$, which is the same as used for the direct optimizations. The convergence history for the population mean of the cost function and the population minimum of the cost function is shown in Fig. 4.17 and it is concluded that convergence has been obtained. $20 \%$ of the inverse-design computations did not lead to a converged solution and these computations have been given a high value for the cost function.

The parameters and the objectives for the optimum are summarized in table 4.7 which compares the original geometry and the two inverse-design cases discussed in chapter 4 . The optimal mean-swirl distribution is shown in Fig. 4.18. 
Table 4.7: Optimized inverse-design compared with different designs from section 3.6.

\begin{tabular}{|c|c|c|c|c|}
\hline & optimum & original & case 1 & case 2 \\
\hline$f_{\text {hub }}(-)$ & 0.375 & - & - & - \\
\hline$f_{\text {shr }}(-)$ & 0.646 & - & - & - \\
\hline \hline$\kappa_{i}\left(10^{-2}\right)$ & 1.13 & 3.69 & 1.60 & 1.39 \\
\hline$\zeta\left(10^{-2}\right)$ & 4.39 & 3.73 & 4.30 & 4.91 \\
\hline$\chi_{\max }(-)$ & 0.83 & 1.28 & 0.85 & 0.76 \\
\hline \hline$F(-)$ & 2.62 & 4.98 & 2.99 & 2.85 \\
\hline
\end{tabular}

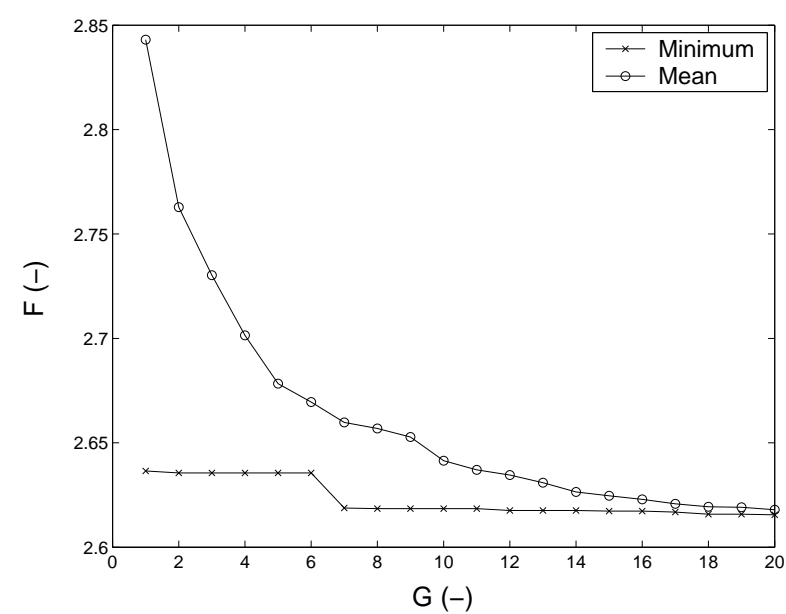

Figure 4.17: Convergence history of the inverse-optimization. Population mean and minimum of cost function as function of generation number.

It is concluded that the mean-swirl distribution is shifted more towards the trailing edge at the hub side, and more towards the leading edge at the shroud side. The optimized inversely-designed impeller and blade angle distribution are shown in Fig. 4.19. The blade angles are between 13 and $30^{\circ}$ and the blade contour at the shroud is markedly shorter than that of the hub contour, resulting from the front loading at the shroud and the aft loading at the hub. The velocity loading and the pressure distribution are displayed in Fig. 4.20. The velocity loading at the shroud has clearly increased when compared to the previous designs. This is not disadvantageous for the maximum velocity loading $\chi_{\max }$, since the maximum still occurs at the hub.

When the objectives are analyzed, it is observed that the optimized geometry has a favorable maximum velocity loading and cavitation inception coefficient, and a slightly higher loss coefficient than the original geometry. For all converged inverse-designs the objectives are compared in Fig. 4.21. If one focuses on the region where the most points are located, i.e. near the optimum, it is seen that the cavitation coefficient is not correlated with the other objectives (horizontal line near the optimum) and that the maximum velocity loading $\chi_{\max }$ and the boundary layer loss coefficient $\zeta$ are negatively correlated. This means that geometries can be obtained from the database which have a lower velocity loading, but at the expense of a higher loss coefficient. 

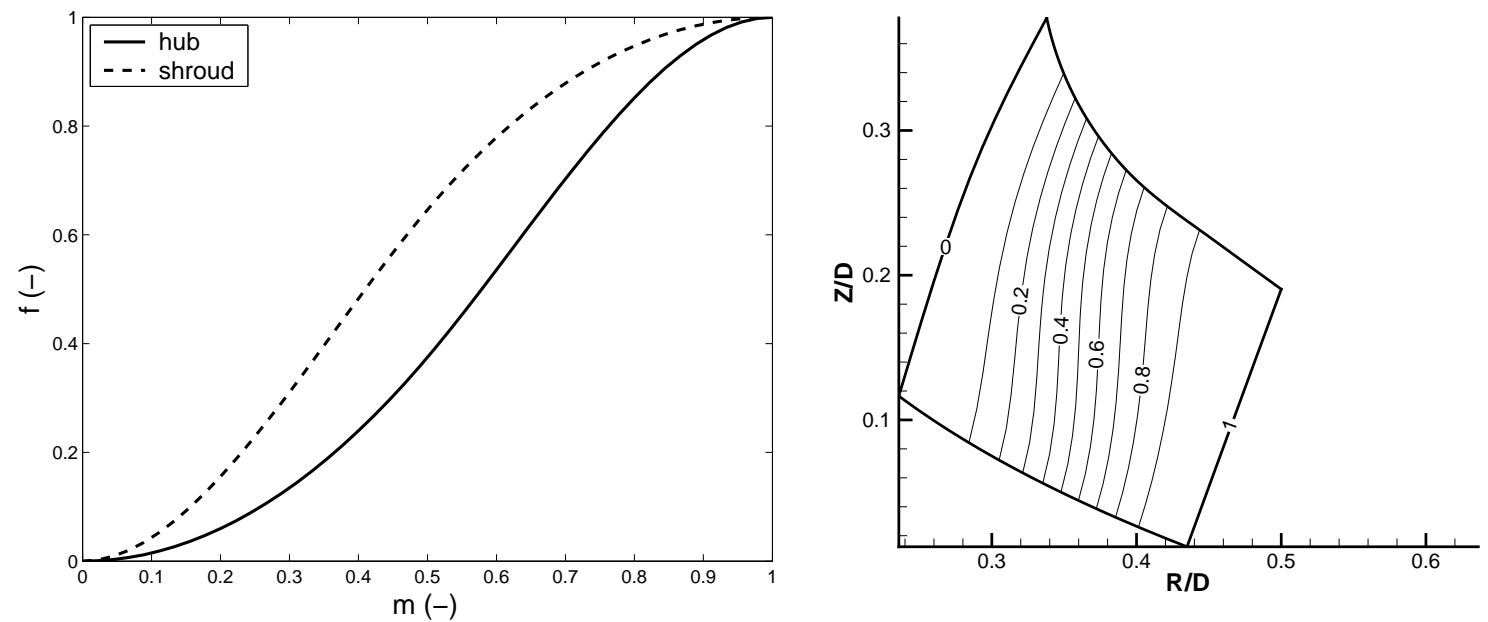

Figure 4.18: Optimized non-dimensional mean-swirl distribution $f$ as a function of the meridional distance (left) and in the meridional plane (right).
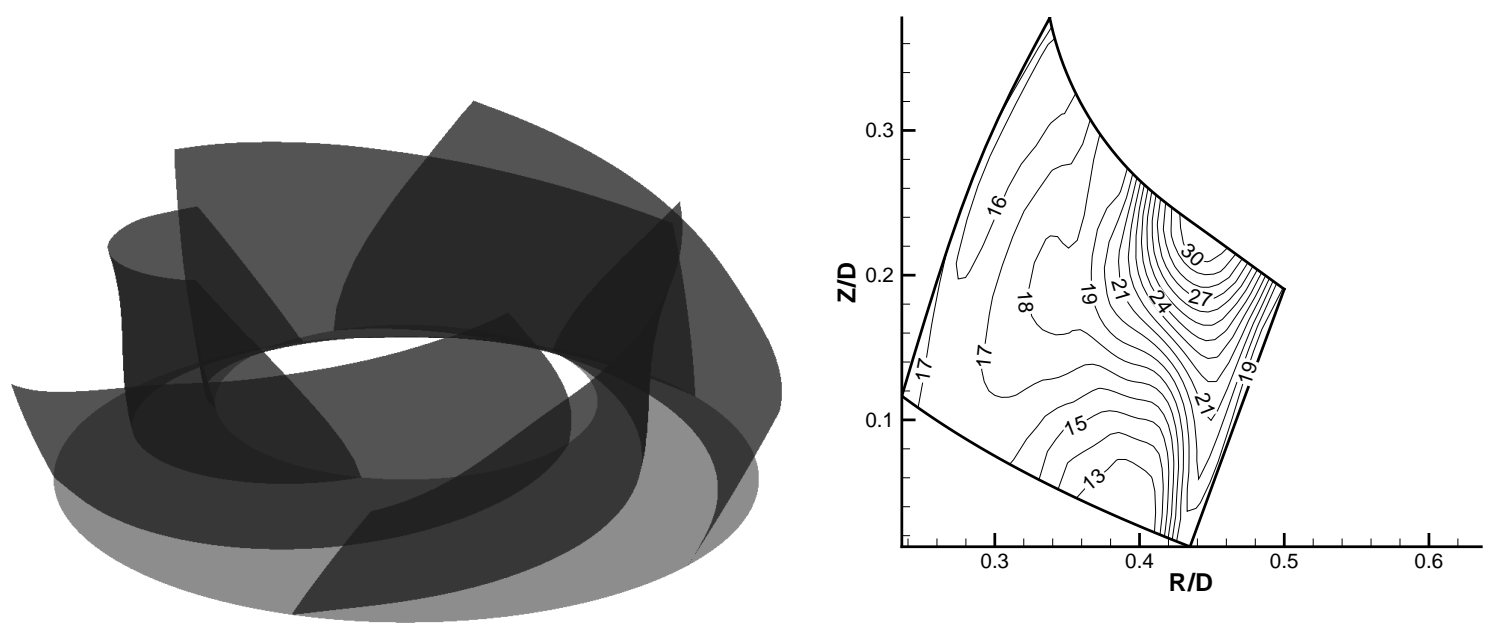

Figure 4.19: Optimized inversely-designed impeller (left) and the associated blade angle distribution in the meridional plane (right). 

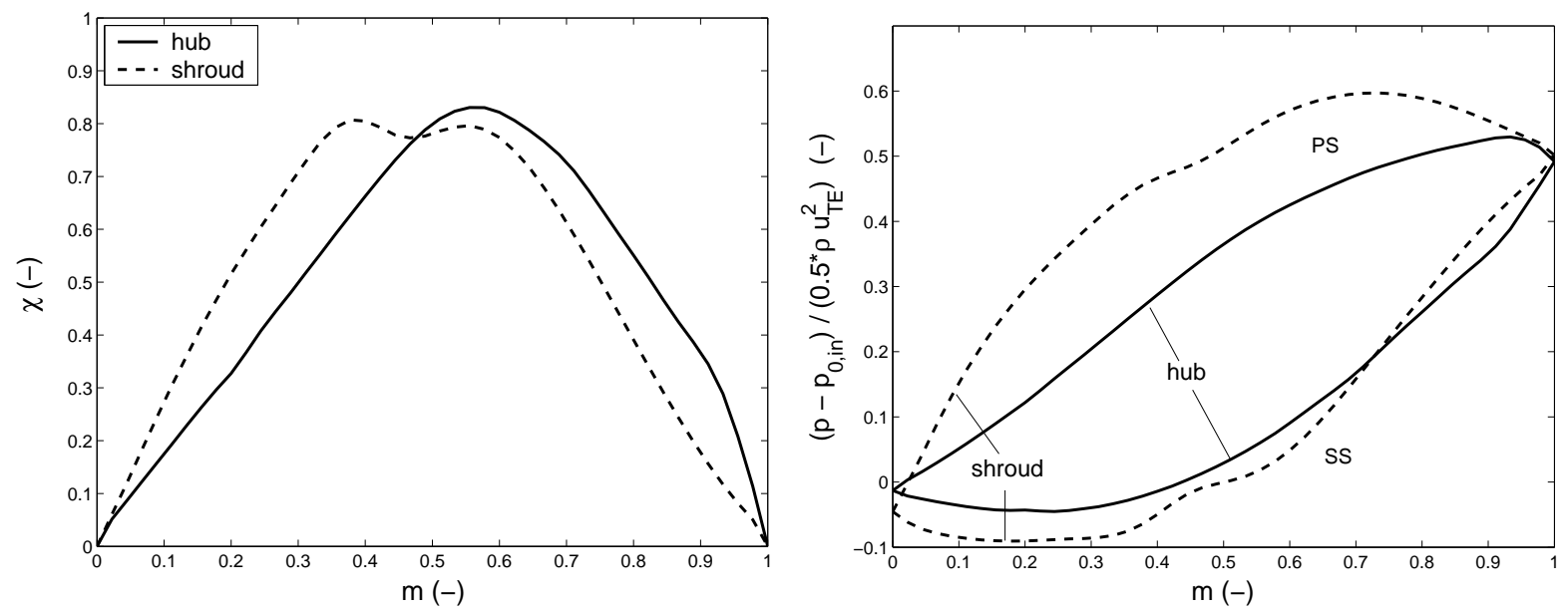

Figure 4.20: The velocity loading on the blades (left) and the non-dimensional pressure distribution (right) for the optimized inverse-design.
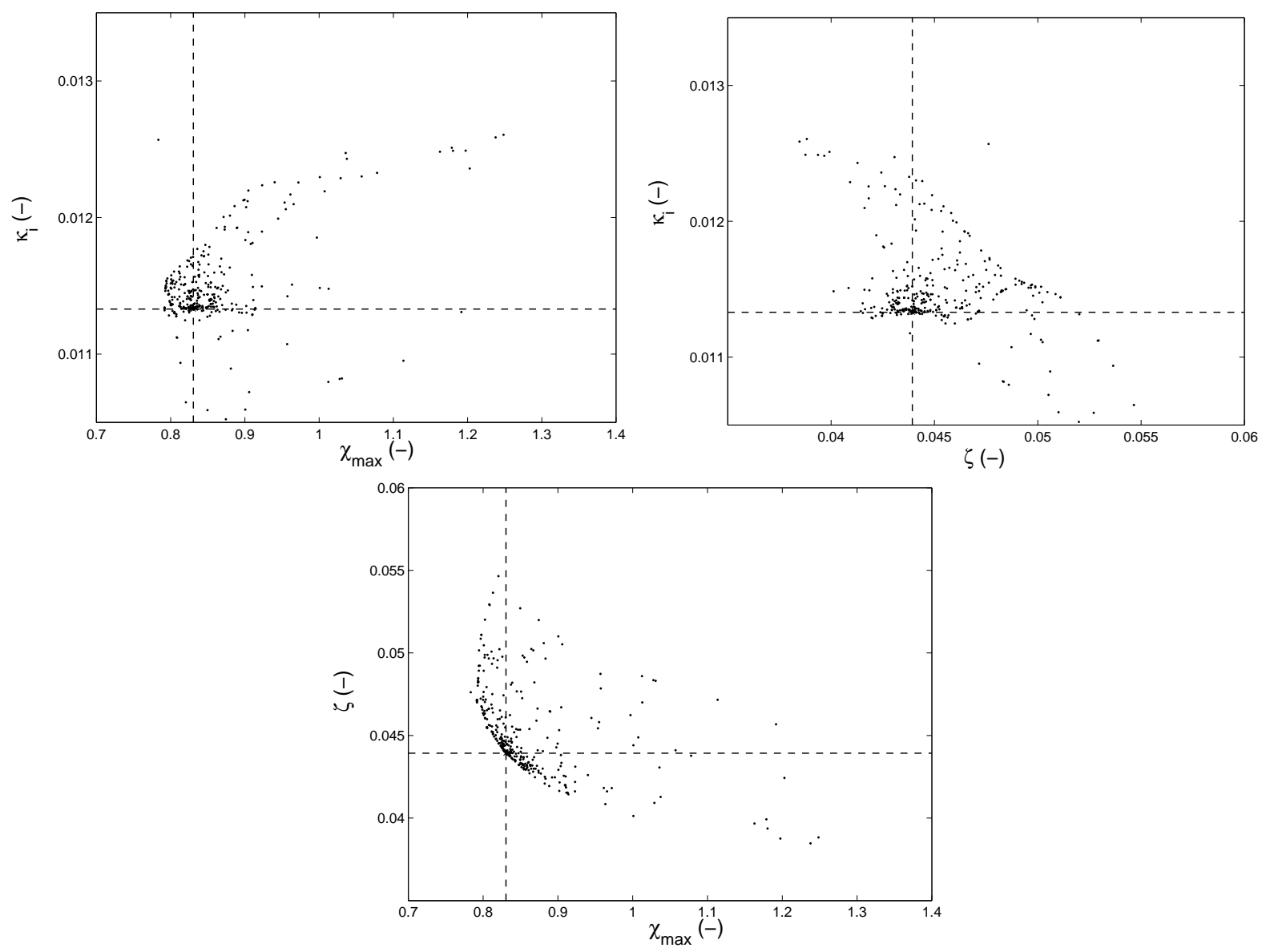

Figure 4.21: Comparison of objectives in the inverse-optimization. The dashed lines indicate the objectives for the optimum. 


\subsection{Discussion}

In this chapter an optimization method for centrifugal pump impellers has been presented. The method uses the Differential Evolution algorithm to solve the minimization problem. This is an evolutionary method in which a population of geometries evolves over a number of generations towards the optimum. The optimization method (generally) yields the global optimum and can deal with non-differentiable cost functions. This method has been verified by application to 3 test functions. The method considers multiple objectives which are summed in a so-called lumped cost function. The objectives are considered at 3 flow rates around the design point, making it a multi-point optimization method.

In section 4.3 the method is applied to the optimization of a radial centrifugal pump impeller. The resulting optimum shows an improvement when compared to the original geometry, mainly on the basis of cavitation inception and cavitation peak width. Additional optimizations with different settings show the influence of the parameterization and the formulation of the cost function. The optimizations considering different parameterizations have shown that further improvements are obtained if the bounded range for the number of blades is increased from $[4,6]$ to $[4,10]$. For the latter bounded range, an optimum impeller is found with 10 blades. An optimization carried out for a different formulation of the head objective resulted in an optimized impeller with 8 blades for a bounded range of $[4,10]$.

In section 4.4 a hybrid method is presented, the so called inverse-optimization. By parameterizing the mean-swirl distribution and formulating a cost function, a inverseoptimization has been carried out of the mixed flow impeller, which was also used in section 3.6. An optimal mean-swirl distribution has been found with a front loading at the shroud-blade intersection and an aft loading at the hub-blade intersection.

An advantage of this hybrid approach is that it is possible to add geometrical parameters, like the number of blades or meridional shape, to the optimization procedure. This is not possible for the conventional inverse-design method. A disadvantage of inverseoptimization is that only the performance at design conditions is considered, whereas for the direct optimization the performance is considered at several flow rates instead. It is possible to consider off-design performance by a combination of an inverse-design computation at design conditions and direct flow computations for off-design conditions (for the inversely designed impellers). This is not pursued in the current method. Another disadvantage is that the inverse-optimization requires many flow computations, since each inverse-design computation requires typically 50 iterations to obtain a converged solution.

One of the main problems for the current optimization method, both for the direct optimization and the inverse-optimization is that it requires many flow computations. The run time can be reduced significantly however, if a response surface method or artificial neural network would be used to estimate the cost function. By optimizing this approximation of the cost function the number of required flow computations can be reduced drastically. Examples of such approaches can be found for example in [23, 34, 57]. This would speed up the procedure drastically. Especially if more sophisticated flow models, than the potential flow model employed here, would be used, this reduction in number of flow computations becomes very important.

Furthermore, the method considers many objectives, but they are all lumped into 
a single cost function which is then minimized. By performing a true multi-objective optimization, the designer will have a choice of many more geometries based on an analysis of the Pareto front [1]. Therefore, it is worthwhile to consider such a multi-objective optimization in the future. If however a response surface method is used, the Pareto front can be approximated from this representation and a multi-objective optimization would no longer be required. Even with the current optimization method, an engineer can choose a geometry from the large database of geometries acquired in the optimization, and does not necessarily need to choose the geometry with the lowest value of the cost function. 



\section{CHAPTER 5}

\section{PIV-measurements in an optimized impeller}

In this work two design methods have been presented for the design of centrifugal pump impellers, i.e. an inverse-design method in chapter 3 and an optimization method in chapter 4 . Both methods have been applied to impeller design cases, as has been demonstrated in preceding chapters. For these design methods the flow inside the impeller is modeled by the augmented potential flow model, which has been derived in chapter 2 . In the present chapter experiments, carried out in a newly designed facility, are discussed with the aim of validating the computed inviscid flow fields, and gaining more insight in the occurring viscous flow fields inside impellers.

An impeller has been designed with the proposed optimization method, as shown in section 4.3. The optimization method has been selected over the inverse-design method, since the optimization method is a multi-point approach, i.e. it considers the performance at both off-design and design conditions, whereas the inverse-design method is applicable to design conditions only. Furthermore, in the inverse-design method only the blade curvature distribution is determined by the method, whereas for the optimization method additional parameters like the number of blades in the impeller and the meridional geometry are varied as well. For the number of blades in the impeller a bounded range of $[4,6]$ has been selected, whereas the original impeller has 7 blades. This bounded range was selected in order to have a good optical accessibility for the PIV measurements presented in this chapter.

In the optimization process the pump impeller has been optimized, based on global performance parameters, being the pump head $H$, efficiency $\eta$ and NPSH. Therefore, it is desirable to measure these global performance parameters directly in a setup. However, in this study the focus has been on measuring detailed velocity distributions in the impeller by means of Particle Image Velocimetry (PIV) measurements, in order to compare the results of these with the predictions of the potential flow model. The static pump head has also been measured, but efficiency and NPSH have not been measured in the current experimental program.

In this chapter the measurements in the optimized impeller are presented. The outline 
is as follows. Firstly, the experimental setup is presented in section 5.1, followed by the operational aspects in 5.2. A description of the PIV method and the PIV parameters is given in section 5.3. The quality of the measurements is discussed in section 5.4. Subsequently, the measurement results are presented for design and off-design conditions in sections 5.5 and 5.6, respectively. The measured velocity distributions are discussed in section 5.7. Finally, in section 5.8 conclusions and recommendations are given related to the measurements. A detailed description of the performed measurements is given by van Andel [2]. Part of these results have also been presented in [75].

\subsection{Experimental setup}

The optimized impeller has been down-scaled from full scale to model scale by approximately a factor 2 , in order to limit the size of the new experimental setup. The flow coefficient $\phi$ and head coefficient $\psi$ (see chapter 1 ) are retained in the scaling, whereas the Reynolds number has been reduced by the scaling of the impeller size and the lower rotational speed. The optimized impeller has been manufactured in PMMA. It is shown in Fig. 5.1 before and after assembly.
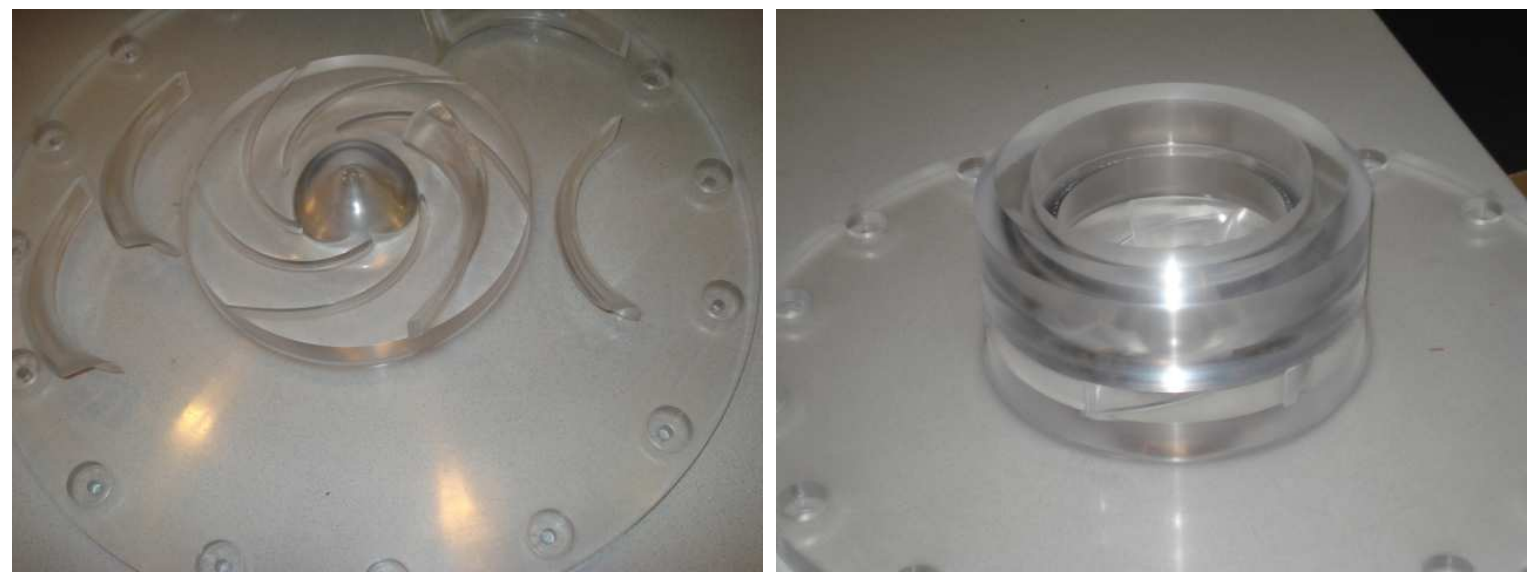

Figure 5.1: Optimized impeller before (left) and after (right) assembly.

The impeller has been placed in a newly designed experimental setup, which is illustrated in Fig. 5.2. The design of the setup is inspired by the experimental setup used by Pedersen [48] for PIV measurements.

The setup consists of a cylindrical Perspex vessel with a diameter of $0.65 \mathrm{~m}$ and a height of $2.0 \mathrm{~m}$. The impeller is located at the top of the setup and it is mounted below a rotating hollow cylinder, which is driven by an electromotor using a belt drive. The flow enters the impeller in upward direction through a central tube, with an inner diameter of $150 \mathrm{~mm}$. By measuring the pressure difference over a calibrated Venturi flow meter inside the central tube, the flow rate is acquired. By changing the opening of a spring, which is located at the bottom of the central tube, the flow rate can be adjusted separately from the rotational speed of the impeller. This spring thus acts as a control valve, which is adjusted with a control valve wheel. A flow straightener, positioned upstream of the 


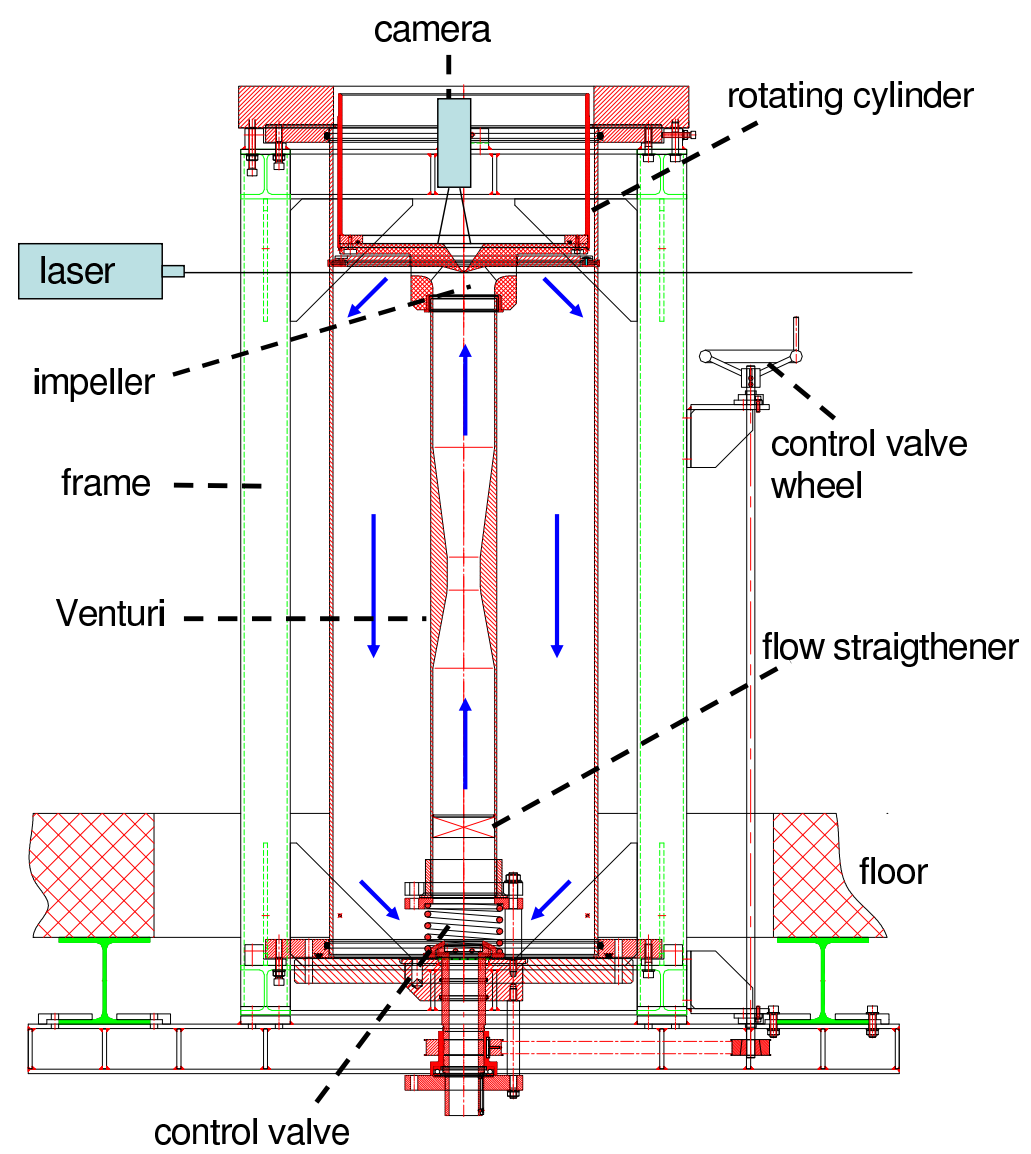

Figure 5.2: Sketch of the experimental setup. The arrows indicate the direction of the flow. The impeller, attached to the rotating cylinder, is rotating; the other parts shown are stationary.

Venturi in the central tube, is used to establish uniform flow at the inlet of the impeller. A photograph of the realized experimental setup is shown in Fig. 5.3.

The operational aspects are discussed in the next section. For more details of the experimental setup the reader is referred to [2].

\subsection{Operational aspects}

In this section the operational aspects of the experimental setup are discussed. This includes an analysis of the Venturi characteristics, a description of the $Q-\Omega$ working envelop and determination of the static pump head.

\subsubsection{Venturi flow meter}

A Venturi flow meter has been manufactured for the measurement of the flow rate $Q$. The configuration is based on general rules for design of Venturis [20,38]: the converging section upstream of the throat of the Venturi has an angle of $10.5^{\circ}$ and the diverging section downstream of the Venturi has an angle of $7.5^{\circ}$. 


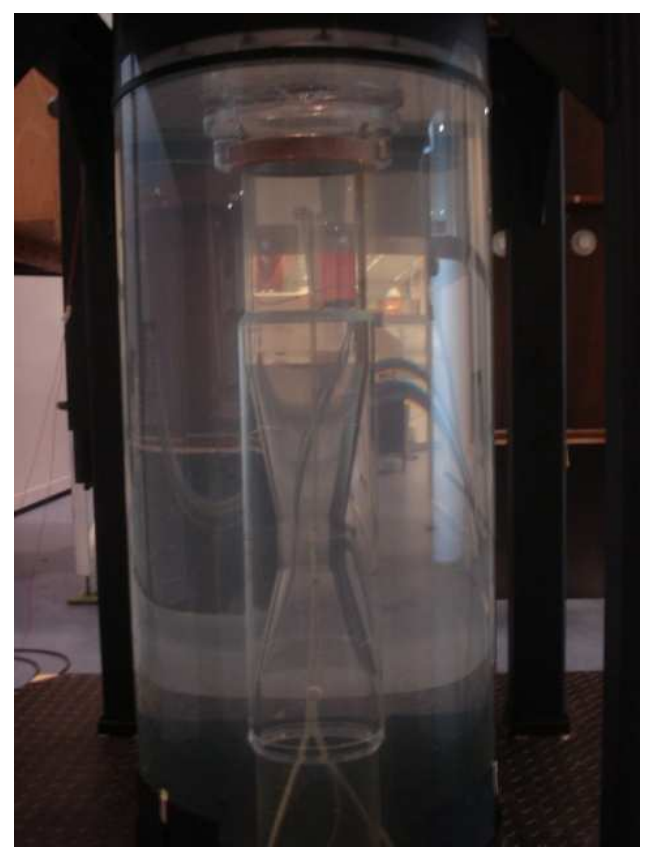

Figure 5.3: Photograph of the experimental setup.

The Venturi tube has been calibrated in a special calibration facility in Aachen, Germany. A picture of the Venturi in the calibration facility is shown in Fig. 5.4 along with a schematic view. The flow rate $Q$ is based on the measurement of the pressure difference $\Delta p$, either for pressure taps 1 and 2 (see Fig. 5.4), or for pressure taps 2 and 3. Only one pressure tap is inserted per section, since the flow is expected to be rotationally symmetric. However, some authors advise to use four or more pressure taps per section in order to obtain a more accurate average pressure at each section [20].

The calibration curves are displayed in Fig. 5.5. A quadratic fit is employed for both curves, since a quadratic dependency between pressure drop and flow rate is expected from the Bernoulli equation. The pressure loss $\Delta p_{\text {loss }}$ inside the Venturi tube is quantified by a loss coefficient $k$, based on the average velocity $v_{2}$ in the narrowest section of the Venturi

$$
\Delta p_{\text {loss }}=\frac{1}{2} k \rho v_{2}^{2}
$$

The loss coefficient based on pressure measurements at taps 1 and $2, k_{12}$, is smaller than the loss coefficient for taps 2 and $3, k_{23}$. This difference is caused by the higher losses in the diverging section of the Venturi. From Fig. 5.5 it is observed that for increasing flow rates the loss coefficient $k_{12}$ decreases in magnitude. This observed trend, however, is smaller than the measurement uncertainty. The loss coefficient $k_{23}$ remains rather constant, as expected.

After the central tube had been inserted into the actual setup, the flow rates were measured based on the two calibration curves for the pressure differences. It became apparent that there was a small difference between the flow rate $Q_{12}$ predicted from taps 1 and 2 and $Q_{23}$ predicted from taps 2 and 3, especially for relatively low flow rates. This 

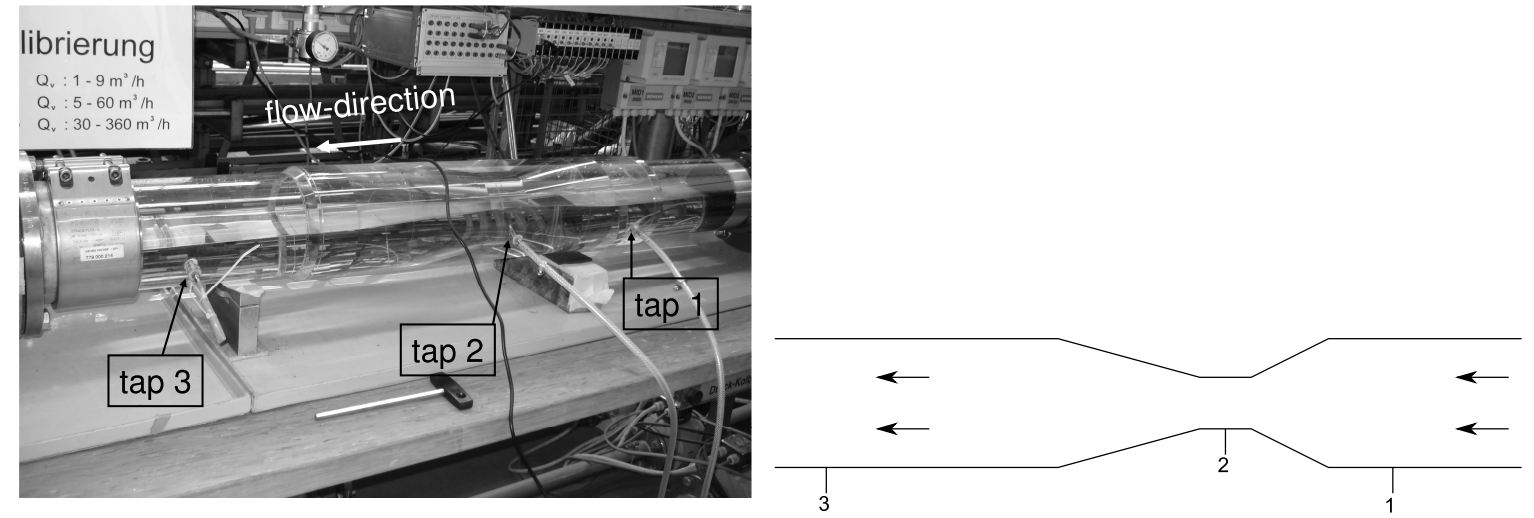

Figure 5.4: Locations of pressure taps 1, 2 and 3 in the Venturi. A picture of the Venturi in the calibration facility is shown on the left; a schematic view is shown on the right.
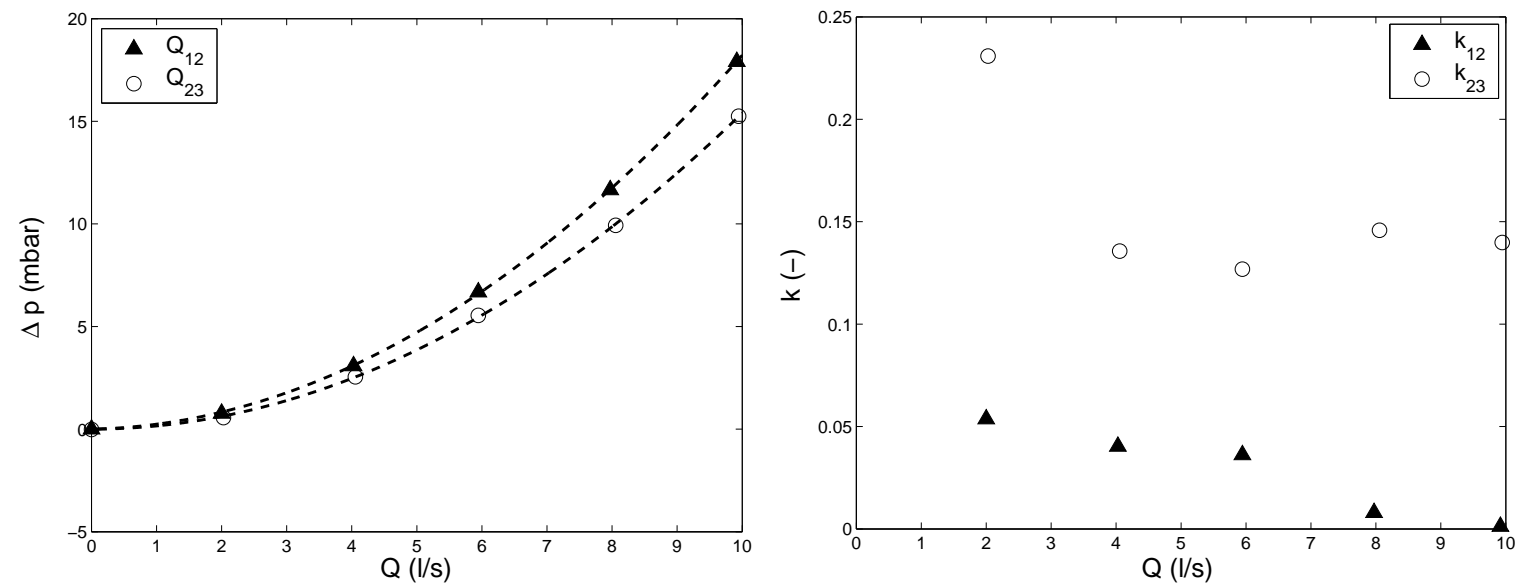

Figure 5.5: The venturi calibration curves (left) and the pressure loss coefficients as function of the flow rate.

is illustrated in Fig. 5.6, where the ratio of both predicted flow rates, $\alpha$, is plotted as a function of the flow rate $Q_{12}$

$$
\alpha=\frac{Q_{23}}{Q_{12}}
$$

Note that in the calibration the relation between $\Delta p_{12}$ and $\Delta p_{23}$ is determined such that $\alpha=1$. The fact that $\alpha \neq 1$ in the realized setup must be due to differences in the configuration between calibration setup and experimental setup. The differences for flow rates around the design flow rate $Q_{d}$, are in the order of $3-4 \%$ and for lower flow rates they are even larger. The main difference between the configuration in the calibration and that in the experimental setup is that an impeller is present in the experimental setup, relatively close to pressure tap 3. Since the influence of the impeller will be smallest for pressure taps 1 and 2, the flow rate is determined from these pressure taps $\left(Q_{12}\right)$. 


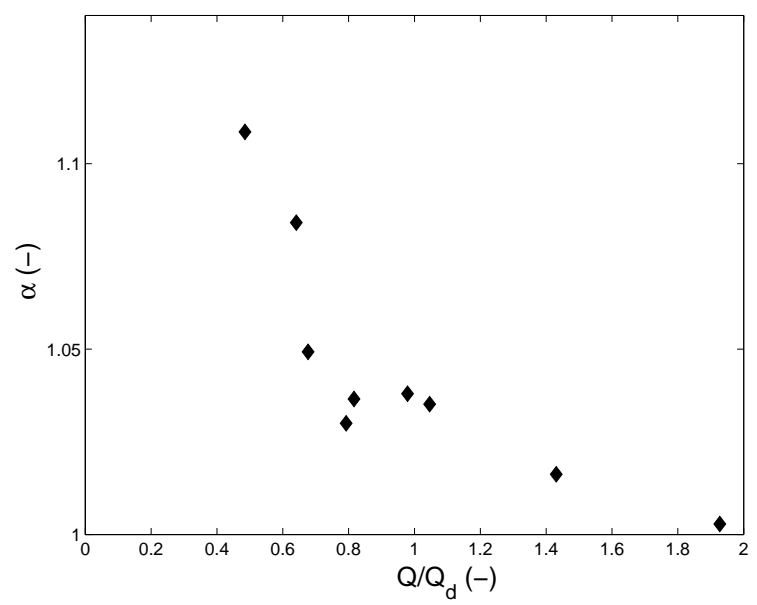

Figure 5.6: The ratio $\alpha=Q_{23} / Q_{12}$ of predicted flow rates as function of the dimensionless flow rate $Q_{12} / Q_{d}$.

\subsubsection{Operating range of the experimental setup}

The experimental setup has been designed for a rotational speed range of $30-600 \mathrm{rpm}$. However, at speeds above 200rpm air bubbles entered the setup due to sloshing at the water-air interface near rotating parts, see Fig. 5.7. These bubbles influence the impeller performance and prevent the generation of a homogenous light sheet in the impeller (each bubble casts a shadow behind it). Therefore, the operating range at which PIV measurements can be performed in the current state of the test facility is $30-200 \mathrm{rpm}$.

The so-called system resistance of the setup has been estimated during the design of the setup, such that the desired flow rates could indeed be realized by the scaled impeller. In this analysis the pressure loss of components like the Venturi, flow-straightener, the central tube and the pressure loss at the impeller exit have been considered. It was concluded that the pressure losses in the system are sufficiently small and that the impeller can realize the desired flow rates.

The flow rate $Q$ is a function of the rotational speed $\Omega$. The control valve, upstream of the Venturi (see Fig. 5.2), is also utilized to control the flow rate $Q$ separately from the rotational speed $\Omega$. At several rotational speeds $\Omega$ the non-dimensional flow rate, i.e. $\phi$, has been measured for two situations, one in which the spring is opened as far as possible (minimum resistance), and one in which it is closed as far as possible (maximum resistance). The resulting working envelop is plotted in Fig. 5.8. It is concluded that the operating envelop is in the range of $0.3 \phi_{d}<\phi<1.9 \phi_{d}$, where $\phi_{d}$ is the flow coefficient at design flow rate. This range is largely independent of the rotational speed and therefore is expected to be similar at rotational speeds larger than 200rpm.

\subsubsection{Pressure drop over the impeller}

The pressure tap close to the inlet of the pump, i.e. pressure tap 3 (see Fig. 5.4), can be utilized to measure the static pressure drop over the impeller. The outlet of the impeller 


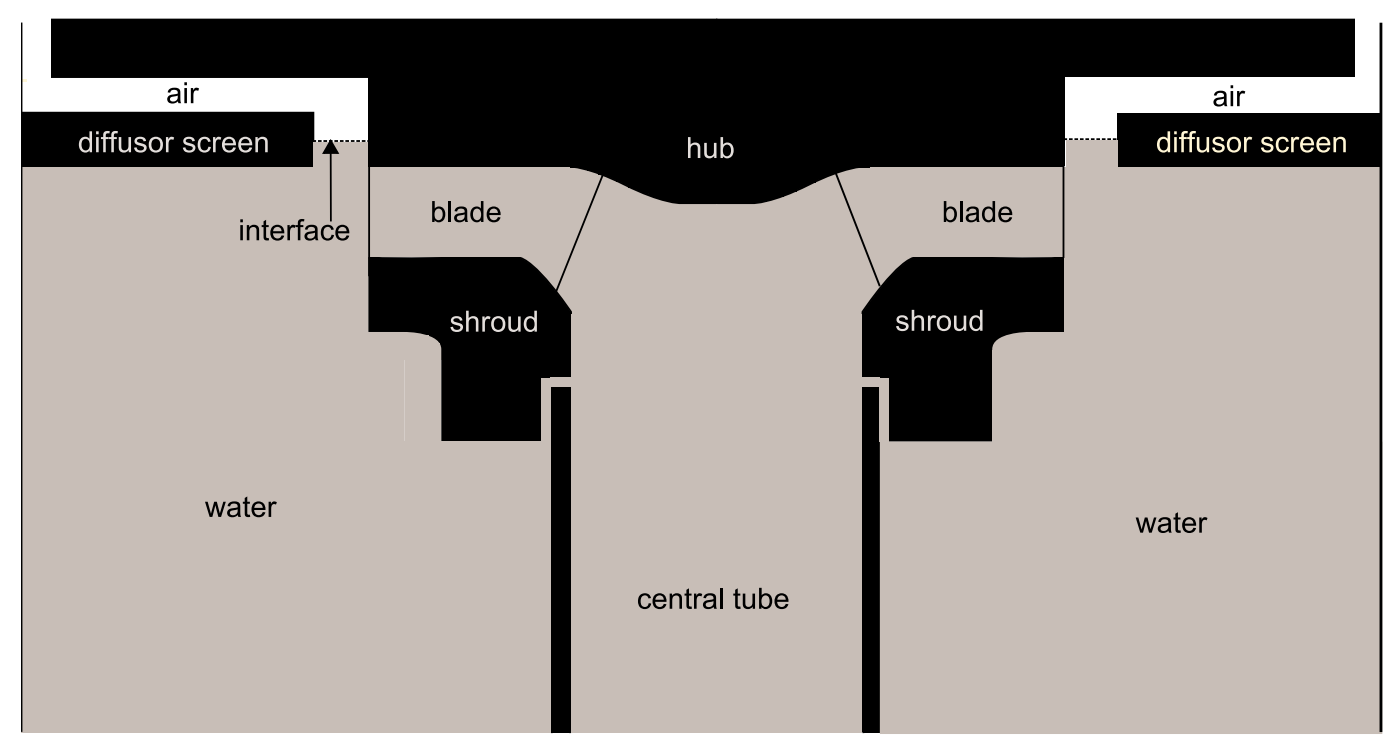

Figure 5.7: The water-air interface. Zoomed-in sketch of the experimental setup around the impeller. The hub, blades and shroud are rotating parts, the other parts are stationary. Above rotational speeds of $200 \mathrm{rpm}$ air bubbles will enter the setup at this interface due to sloshing effects.

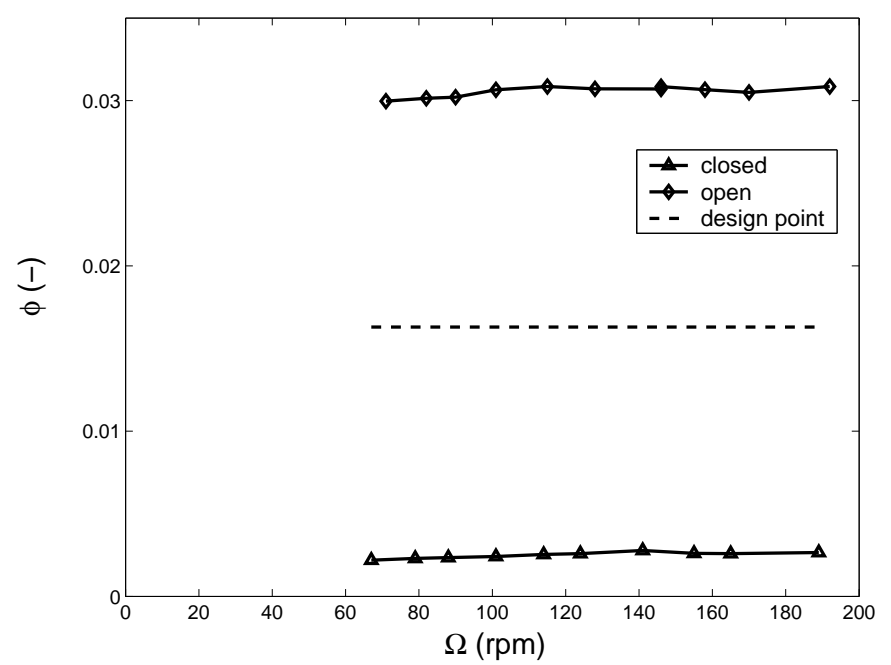

Figure 5.8: $\Omega-\phi$ working envelop. The flow coefficient $\phi=Q /\left(\Omega D^{3}\right)$ as a function of the rotational speed $\Omega$ for the situation with the spring fully closed and fully open, respectively.

is close to the air-water interface (see Fig. 5.7), which is at atmospheric pressure $p_{a t m}$, and therefore it is assumed that the pressure at the pump outlet is at atmospheric pressure. By measuring the pressure difference $\Delta p_{34}$ between pressure tap 3 and a reference pressure $p_{4}$, with $p_{4}=p_{a t m}$, the static pressure drop $\Delta p$ over the impeller is measured. The static pump head $H_{\text {stat }}=\Delta p /(\rho g)$ can therefore be determined.

Note that the static pump head differs from the conventional pump head, since the change in static pressure is considered and not the change in stagnation pressure. By 


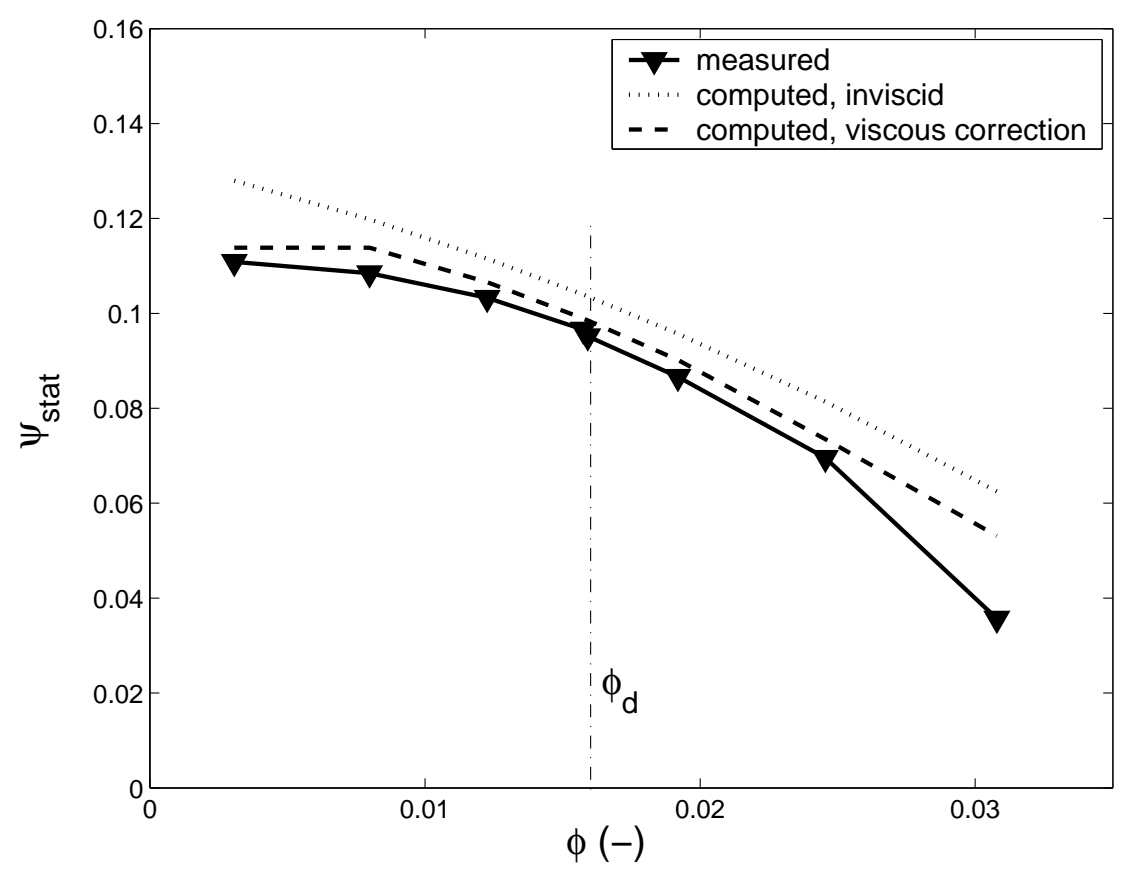

Figure 5.9: Measured static pump head coefficient $\psi_{\text {stat }}=g H_{\text {stat }} /\left(\Omega^{2} D^{2}\right)$ as a function of the flow coefficient $\phi=Q /\left(\Omega D^{3}\right)$ compared with results from potential flow model predictions with and without viscous correction. Measurements carried out at $\Omega=100 \mathrm{rpm}$.

measuring $\Delta p_{34}$ as a function of the flow rate $Q$ a $Q-H_{\text {stat }}$ curve has been determined; it is compared to the computed curves in Fig. 5.9. Note that the dimensionless values are displayed, i.e. the flow coefficient $\phi=Q /\left(\Omega D^{3}\right)$ and the head coefficient $\psi_{\text {stat }}=$ $g H_{\text {stat }} /\left(\Omega^{2} D^{2}\right)$. For the computations an inviscid-flow curve is shown and one corrected for viscous losses by using Eqn. (2.27) (see section 2.3). As expected, the measured values are somewhat lower than the inviscid-flow predictions, due to the viscous losses that occur in the flow through the impeller. These losses are somewhat higher than predicted by the viscous-flow correction.

The difference between measurements and computations is smallest near the design point, indicating that the impeller is well designed. In the design point the measured static pump head deviates $7 \%$ from the computed inviscid-flow pump head and $3 \%$ from the computed pump head, which has been corrected for viscous-flow losses. 


\subsection{Particle Image Velocimetry}

In order to measure the velocity field inside impellers it is advantageous to make use of non-intrusive measurement techniques. The most frequently used techniques used for this purpose are Particle Image Velocimetry (PIV) and Laser Doppler Velocimetry (LDV). In the present study PIV has been selected as measurement technique, since it is a wholefield measurement technique, whereas LDV is a single point measurement technique. The PIV measurements presented here are 2D in nature, that is only the in plane velocity components are measured. Using Stereo PIV (SPIV), it is also possible to determine the out-of-plane velocity component utilizing a second camera. However, his has not been pursued in this research. The camera is stationary and hence the absolute velocity components are measured, whereas it is sometimes convenient to use a co-rotating camera to measure the relative velocity directly, as is done for example in [69]. A major advantage of PIV is that instantaneously a complete velocity vector field is obtained and detailed instantaneous flow phenomena can be revealed. This was the main reason for the selection of PIV as flow measurement method in this project.

In this section the PIV measurement technique is discussed and general guidelines for good PIV measurements are presented. Firstly, a literature overview of PIV measurements performed in turbomachine impellers is given in section 5.3.1. Secondly, the working principle of PIV is introduced in 5.3.2. General rules of thumb for PIV are addressed in section 5.3.3. Furthermore, the parameters used in the PIV measurements are presented.

\subsubsection{Literature overview}

The purpose of this section is to give an overview of the literature on PIV measurements carried out in turbomachine impellers. The main observations from these measurements are presented. They show the interesting possibilities of PIV measurements in turbomachine impellers.

Visser [69] carried out PIV measurements in a low specific speed radial pump impeller. From the PIV measurements it was concluded that the measured flow was predominantly of a potential flow type over a wide range of flow rates, i.e. low values of the relative velocity at pressure side and high values of the relative velocity at the suction side of the blades. Wuibaut et al. performed PIV measurements in the radial SHF impeller in air $[77,78]$. In these papers the focus is on PIV measurements in the outer part of the impeller and in the diffuser. In both papers a jet-wake structure is observed near the shroud. The wake-like region, which is a region of low velocity but not of back-flow, is located at the suction side. Pedersen $[48,49]$ executed PIV measurements in a radial impeller with 6 blades and reports predominantly identical flow in all channels, in which the flow follows the blades. A wake-like region is observed at the suction side of the blades. At offdesign conditions, i.e. at a flow rate of $25 \%$ of the design flow rate, a two-channel flow pattern is observed, in which one channel shows a flow structure similar to that at design conditions, and the other is exhibiting stall at the inlet. Krause et al. [45] performed time-resolved PIV measurements in a radial pump impeller and focussed on unsteady flow phenomena occurring at off-design flow rates. For decreasing flow rates a spatially stable stalled region was observed. Further reduction in flow rate led to the occurrence 
of rotational stall inside the impeller. Choi et al. [12] executed PIV measurements in two unshrouded impellers (contrary to the shrouded impellers considered here), a sixbladed impeller with straight vanes and a four-bladed impeller with backward curved blades. For both impellers the large tip leakage flows result in a complex flow behavior dominated by reverse flow and secondary flow effects, even at design conditions. Sinha et al. [60] carried out PIV measurements in a centrifugal pump and they studied the flow in the outer part of the impeller and the vaned diffuser at low flow rates. They observed rotating stall in one diffuser channel, which extended to two channels when the flow rate was further decreased. Frölig et al. [36] performed Stereo PIV (SPIV) measurements in three unshrouded impellers at both off-design and design conditions. The measured flow patterns are dominated by a strong interaction between secondary flow and leakage flow through the gap.

\subsubsection{PIV principle}

The goal of PIV measurements is to measure velocity distributions in a flow. For this purpose small tracer (or seeding) particles are added to that flow. By determining the particle displacements during a fixed time interval, the particle velocities $\boldsymbol{v}_{p}$ can be determined. If the seeding particles are small and have a small density difference with the fluid, the slip between seeding particles and fluid can be neglected. This means that the local fluid velocity $\boldsymbol{v}_{l}$ is equal to the average particle velocity $\boldsymbol{v}_{p}$. Therefore, by choosing appropriate seeding particles, the fluid velocity distributions can be determined from the particle displacements.

A fixed plane is illuminated with laser light, for which a Nd:YAG laser is frequently utilized. A digital camera is then used to obtain two snap shots, frequently called an image pair, with a relative short time interval $\Delta t$ apart. The particles scatter the laser light and can be observed in the two camera images. The key problem is to find a typical particle displacement $\Delta s$ such that the in-plane liquid velocity can be computed

$$
\boldsymbol{v}=\frac{\Delta s}{\Delta t}
$$

The magnification factor $M_{0}$ gives the ratio between the image size on the chip and the real length scale

$$
M_{0}=\frac{\Delta x_{\text {chip }}}{\Delta x_{\text {real }}}
$$

The magnification factor is needed to determine the real particle displacement $\Delta \boldsymbol{s}=$ $\Delta \boldsymbol{s}_{\text {chip }} / M_{0}$.

In order to obtain a velocity vector field the image area is divided into smaller areas, so-called interrogation areas (IAs), for each of which a velocity vector is to be determined. The displacement of particles inside an interrogation area thus leads to the determination of a velocity vector for this interrogation area. For each interrogation area the light intensities between two corresponding images are correlated. The average particle displacement $\Delta \boldsymbol{s}$, is the value for which the correlation $R(\Delta \boldsymbol{s})$ is maximal. The correlation 
function $R(\Delta s)$ is defined by

$$
R(\Delta \boldsymbol{s})=\int I_{1}(\boldsymbol{x}) I_{2}(\boldsymbol{x}+\Delta \boldsymbol{s}) d \boldsymbol{x}
$$

where $I_{1}$ and $I_{2}$ are the light intensity distributions for the first and second camera image, respectively; $\boldsymbol{x}$ is the interrogation location. The larger the number of interrogation areas is, the higher the spatial resolution of the PIV measurement is. A typical PIV setup is shown in Fig. 5.10.

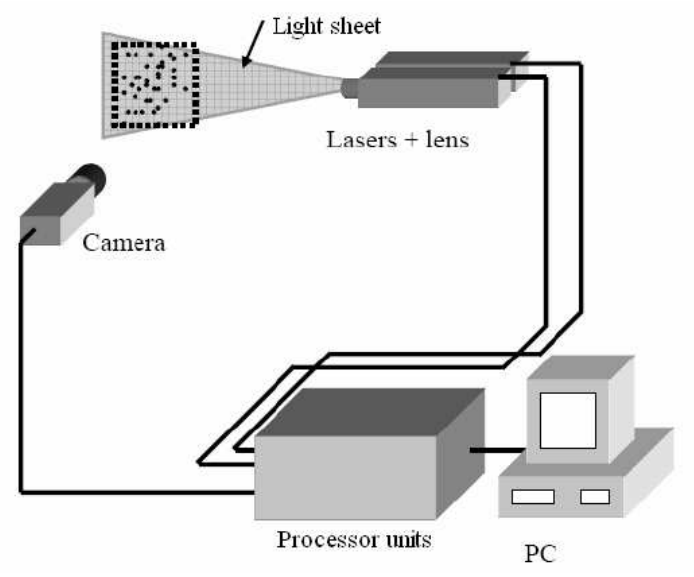

Figure 5.10: A typical PIV setup configuration. Original picture taken from Deen [21].

By considering the displacement at which the maximum correlation occurs, an in-plane displacement vector is found, with its (two) components expressed in pixels, resulting in an error in the particle displacement of the order of 1 pixel. In order to obtain a more accurate particle displacement vector, a fit of the correlation function near the peak is made using a so-called sub-pixel interpolator. The Gaussian sub-pixel interpolator is preferred over centroid or quadratic sub-pixel interpolators, since it has been shown to give the best results [73]. By using such a Gaussian sub-pixel interpolation method the error in the particle displacement can be reduced to typically 0.1 pixel or even less [73]. If relatively small particles are used, typically with an image diameter of less than one pixel, no good sub-pixel interpolation can be performed and a bias towards integer displacements may occur. This phenomenon is referred to as peak or pixel locking. An investigation on peak-locking effects is given, for example, by Christensen [13].

The choices of appropriate seeding particles, interrogation area size and time step are very important in PIV measurements. General guidelines for good PIV measurements are therefore discussed next.

\subsubsection{PIV parameters}

In this section the guidelines for PIV measurements are presented and the selected settings for the measurements are elucidated. These guidelines are summarized for example in [56]. Firstly, parameters related to the employed laser and camera are discussed. This includes 
the numerical aperture, the focal depth and the image calibration. The time interval selected for the PIV measurements is discussed next. Subsequently, particle properties are addressed, including particle image size, concentration and slip. Finally, the processing options are treated and an overview of the measurement program is given.

\section{Laser and camera}

A PIV system from LaVision has been utilized for the measurements. It consists of a double-pulsed Nd:YAG laser with a wavelength of $532 \mathrm{~nm}$, which is employed to illuminate a horizontal plane in the impeller. Measurements have been performed at two heights $z$ in the impeller, i.e. at $z / b=0.35$, close to the hub, and at $z / b=0.85$, i.e. close to the shroud, as illustrated in Fig. 5.11. Here $b$ is the width of the impeller at the trailing edge. A digital camera, with a resolution of $1024 \times 1280$ pixels, is used for image acquisition. The

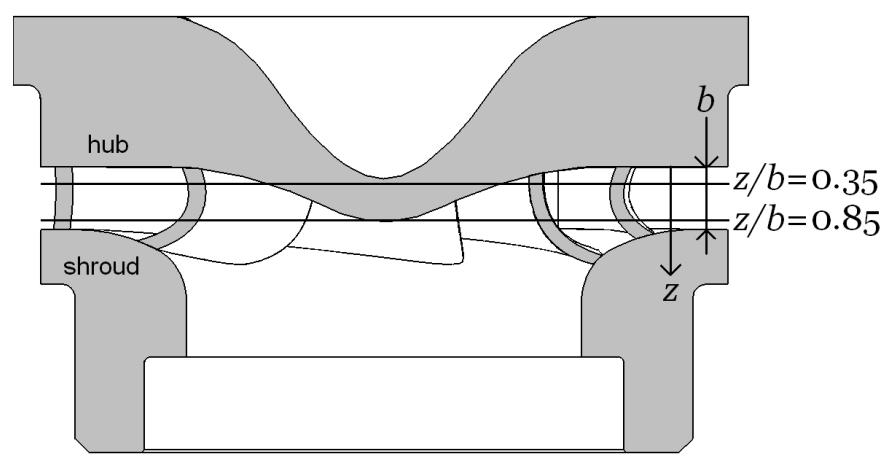

Figure 5.11: The horizontal light-sheets, which are illuminated with a laser, indicated in a cross-section of the impeller. One plane is located near the hub, $z / b=0.35$, and one near the shroud, $z / b=0.85$.

camera is located inside the rotating cylinder, viewing down at the impeller (see Fig. 5.2). During each impeller revolution an image pair is acquired at the same angular position in the impeller by using a trigger system coupled to the position of the rotating cylinder.

\section{Numerical aperture}

For image acquisition the diaphragm of the camera has to be set, resulting in a value of $f^{\#}$, the numerical aperture of the camera. The numerical aperture is defined as the ratio between focal distance $f$ and the lens aperture $d_{l}$

$$
f^{\#}=\frac{f}{d_{l}}
$$

The numerical aperture influences the light intensity of the image, the particle image diameter and the focal depth. Initially a value of $f^{\#}=8$ was considered, but this led to relative poorly illuminated images, which resulted in a relatively high number of spurious vectors in the obtained PIV vector fields. By decreasing the numerical aperture to $f^{\#}=4$, an image pair was obtained with sufficient light intensity, such that good vector fields, with a limited amount of spurious vectors, could be obtained. The influence of numerical aperture on particle image size and focus depth is discussed in subsequent sections. 

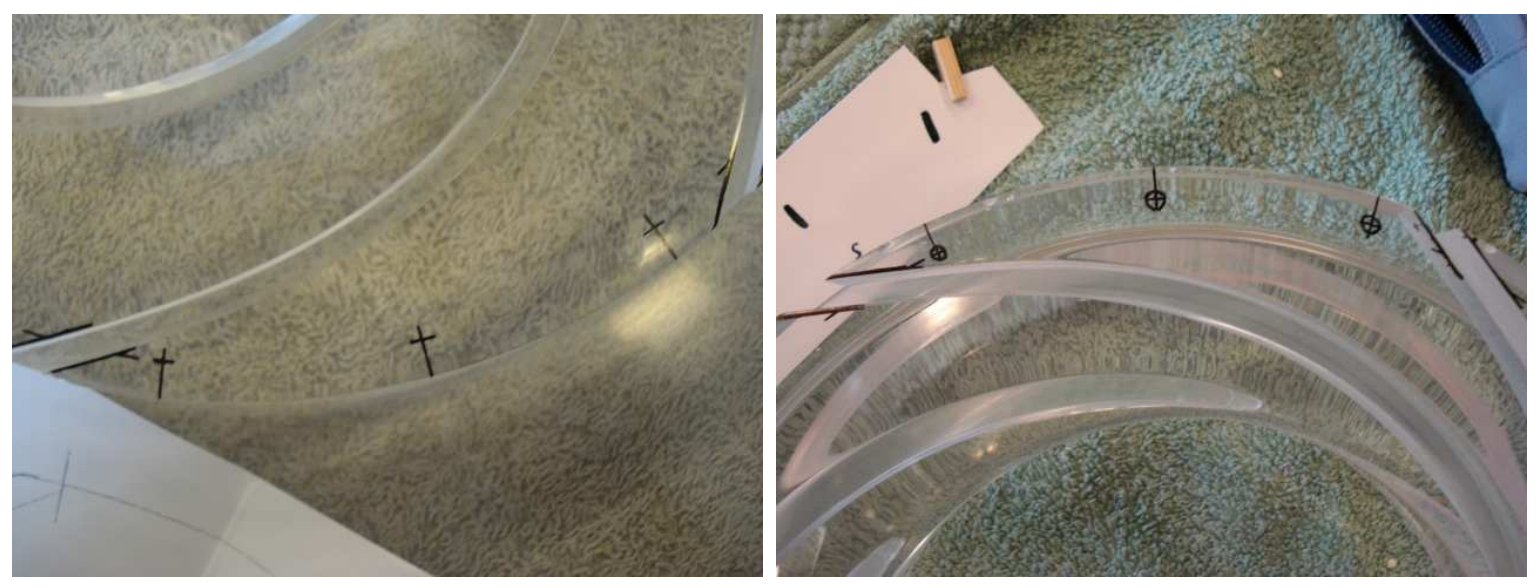

Figure 5.12: Markings on hub (left) and shroud (right) used for PIV image calibration.

\section{Focal depth}

The laser optics are selected such that the light sheet thickness $z_{0}$ inside the impeller is around $2 \mathrm{~mm}$. The focus depth $\delta z$ of the camera is determined from [56]

$$
\delta z=4\left(1+\frac{1}{M_{0}}\right)^{2} f^{\#^{2}} \lambda
$$

The camera has been placed at such a distance from the impeller that effectively more than one channel could be viewed. This resulted in a magnification factor of $M_{0}=0.065$. Using this value, the focus depth for the measurements is computed at $9 \mathrm{~mm}$, which is roughly four times larger than the light sheet thickness, caused by the relative low magnification factor $M_{0}$. In setting up PIV measurements the aim usually is to match $z_{0}$ and $\delta z$, but this is not an exact requirement.

\section{Image calibration}

In order to determine the absolute and relative velocity components in the impeller, an image calibration has to be performed. For this purpose, calibration markings have been drawn on both the hub and the shroud surface of one of the impeller channels, as shown in Fig. 5.12. The image is calibrated for each laser-light sheet plane between hub and shroud based on a linear interpolation from the hub and shroud markings. The image calibration is first of all used to determine the magnification factor $M_{0}$ and therewith for the calculation of the absolute velocity $\boldsymbol{v}$ from the measurements. Secondly, the calibration markings are used to convert the absolute velocity $\boldsymbol{v}$ to the relative velocity $\boldsymbol{w}$ by (see also section 1.2)

$$
\boldsymbol{w}=\boldsymbol{v}-\Omega \times \boldsymbol{r}
$$

where $\boldsymbol{r}$ is the position vector and $\boldsymbol{\Omega}$ is the angular speed vector of the impeller. 


\section{Time interval}

The time interval $\Delta t$ between two corresponding images is chosen such that the displacement $\Delta x$ of the seeding particles is close to a quarter width of an interrogation area $D_{I A}$ $[56]$.

$$
\Delta x<0.25 D_{I A}
$$

A larger time step would mean that too many particles leave the interrogation area within in one time interval, whereas a smaller time step will mean a smaller displacement, and hence a larger relative error in the velocity. A time step of $\Delta t=600 \mu s$ has been selected. Only near the trailing edge and close to the blades, the particle displacement slightly exceeds the range specified in Eqn. (5.9).

The out-of-plane displacement $\Delta z$ is also of importance for similar reasons as the inplane displacement: the out-of-plane velocity component $v_{z}$ needs to be small enough to avoid that many particles leave the laser sheet, with sheet thickness $z_{0}$, within the time step $\Delta t$

$$
\Delta z=v_{z} \Delta t<0.25 z_{0}
$$

From the computed flow field it is concluded that this condition is easily satisfied.

\section{Particles and particle image size}

Polyamide particles from Dantec are employed as seeding. These particles have a mean particle diameter of $d_{p}=50 \mu \mathrm{m}$ and a density of $1030 \mathrm{~kg} / \mathrm{m}^{3}$. The particles are distributed fairly homogeneously throughout the setup, although they settle down when the setup is not operational for some time.

The particle image size $d_{t}$ is a combination of the real particle size $d_{p}$ and the diffraction limited spot size $d_{s}$

$$
\begin{gathered}
d_{s}=2.44\left(1+M_{0}\right) f^{\#} \lambda \\
d_{t}=\sqrt{M_{0}^{2} d_{p}^{2}+d_{s}^{2}}
\end{gathered}
$$

where $\lambda$ is the wavelength of the laser light. The particle image size $d_{t}$ is optimal for PIV measurements when it is close to 2 pixels $[73,74]$.

For the measurements the particle image size is computed to be $d_{t}=6.4 \mu \mathrm{m}$ (with $M_{0}=0.065, f^{\#}=4$ and $\left.\lambda=532 \mathrm{~nm}\right)$. The camera pixel size is $6.7 \mu \mathrm{m}$ and therefore the particle image size in pixels is $0.95 p x$. This value is somewhat low, caused by the low value of the magnification number $M_{0}$ and the relatively low value of the numerical aperture $\left(f^{\#}=4\right)$, which was required for good image illumination. However, when inspecting the PIV images the particles are found to be generally larger than 1 pixel, as is seen from Fig. 5.13. The reason for this is not clear, but it might be caused by either a difference in real particle size or imperfect focussing. Peak locking might occur for particles with an image diameter smaller than one pixel. It will be investigated in section 5.4.2 whether this phenomenon occurs in the measurements. 


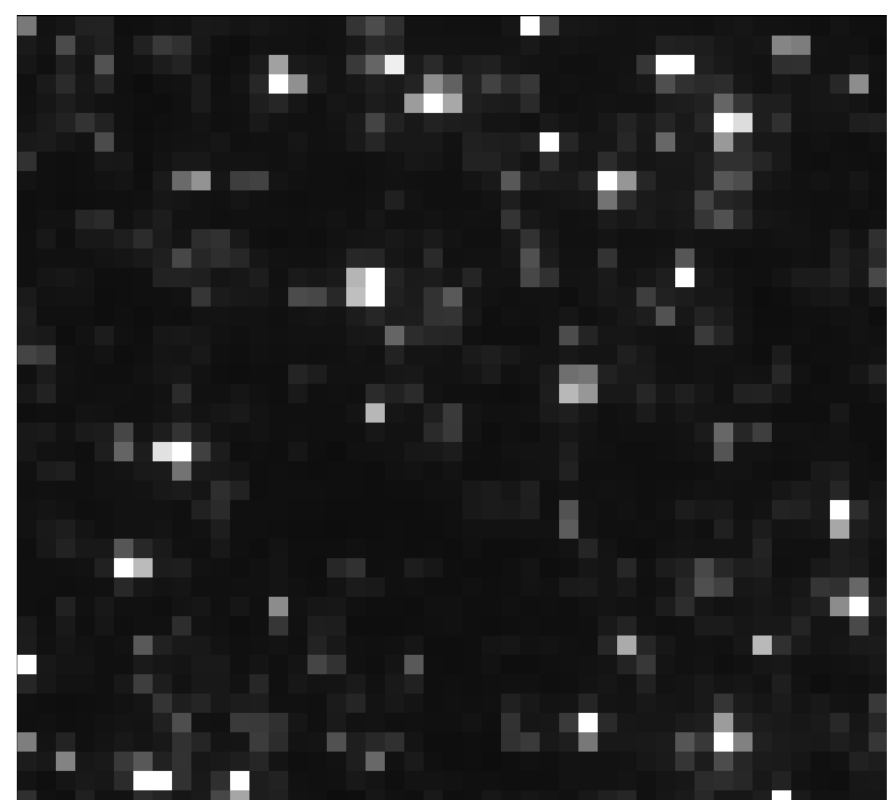

Figure 5.13: A zoomed-in PIV image. The particles are typically somewhat larger than 1 pixel.

\section{Particle concentration}

In order to obtain a distinct correlation for each interrogation area, a number of particles is needed for each interrogation area. For PIV measurements of good quality, the number of particles per interrogation area, $N_{I}$, should satisfy [56]

$$
N_{I}>10
$$

$N_{I}$ can be determined from the particle concentration $C_{n}$ (the number of particles per unit volume) by

$$
N_{I}=C_{n} z_{0} D_{I A}^{2}
$$

The particle concentration $C_{n}$ in turn is obtained from the particle volume concentration $C_{v}$ (volume of particles per volume of fluid) via the particle diameter $d_{p}$

$$
C_{n}=\frac{C_{v}}{V_{p}}=\frac{6 C_{v}}{\pi d_{p}^{3}}
$$

where $V_{p}$ is the particle volume. Particles have been added to the setup, leading to a volume concentration $C_{v}$ of $5 \cdot 10^{-5}$, which should correspond to $N_{I} \approx 17$. However, in the PIV images it has been observed that the actual concentration was lower, since not all particles were homogenously dispersed in the water. Therefore, the volume concentration $C_{v}$ has been increased to $1 \cdot 10^{-4}$, which is a rule of thumb frequently used in PIV measurements. From PIV images it was concluded that equation (5.13) then was satisfied. 


\section{Particle slip}

The assumption in PIV measurements is made that the seeding particles follow the flow and that particle slip can be neglected. In reality particle slip does exist. A rough estimate is made here for the magnitude of the particle slip $v^{\prime}=v_{p}-v$ in the impeller in the horizontal plane, which means that gravity and buoyancy are not considered. An order of magnitude analysis of the slip velocity is performed by assuming equilibrium between the drag force $F_{D}$ and the centrifugal force $F_{C}$

$$
\begin{aligned}
F_{C} & \approx F_{D} \\
V_{p}\left(\rho_{p}-\rho_{l}\right) \Omega^{2} r & \approx \frac{1}{2} A_{p} C_{D} \rho_{l} v^{\prime 2}
\end{aligned}
$$

where $\Omega^{2} r$ is the centrifugal acceleration, $V_{p}$ the particle volume, $A_{p}$ the cross-sectional area of the particle, $C_{D}$ the drag coefficient, $\rho_{p}$ the density of the particles and $\rho_{l}$ the density of the liquid. The drag coefficient $C_{D}$ is a function of the Reynolds number and thus of $v^{\prime}$. For sufficiently low Reynolds numbers, Stokes drag may be assumed

$$
C_{D}=\frac{24}{R e_{d}}=\frac{24 \eta}{\rho_{l} v^{\prime} d_{p}} \quad R e_{d}<0.1
$$

where $\eta$ is the dynamic viscosity of the liquid. This results in the following equation for the slip velocity $v^{\prime}$, which is in accordance with similar considerations $[21,56]$

$$
v^{\prime}=\frac{\left(\rho_{p}-\rho_{l}\right) d_{p}^{2}}{18 \eta} \Omega^{2} r
$$

At design conditions a particle slip is found of $v^{\prime} / u_{t e}=6.3 \cdot 10^{-5}$. This low value of the slip velocity is mainly caused by the small difference in density between the particles and that of the liquid. Therefore particle slip can be neglected.

\section{PIV processing options}

The interrogation area size has been set at 32 by 32 pixels and an overlap of $50 \%$ has been selected. The digital camera has a resolution of 1280 by 1024 pixels and therefore a vector field of 80 by 64 vectors is obtained.

In order to determine the velocity vectors, a multi-pass algorithm with a median filter has been used. Firstly, a correlation is determined based on interrogation areas with a size of 64 by 64 pixels, followed by 2 further passes for interrogation areas of 32 by 32 pixels. This procedure was found to be adequate and additional passes did not lead to further improvement of the results, for more details see [2].

\section{Overview of PIV parameters and measurements}

The PIV parameters that have been utilized for the measurements are summarized in table 5.1. These parameters have been used, unless mentioned otherwise.

Measurements have been performed at a rotational speed of $150 \mathrm{rpm}$ and for two horizontal planes in the impeller (see Fig. 5.11). For each plane measurements have been 
performed for $50 \%, 80 \%, 100 \%, 120 \%$ and $150 \%$ of the design flow rate $Q_{d}$. Furthermore, additional measurements have been executed at $75 \mathrm{rpm}$ to investigate the influence of the Reynolds number on the results. Reproducibility measurements have also been carried out.

Table 5.1: PIV parameters used in the experiments.

\begin{tabular}{|l|l|}
\hline parameter & value \\
\hline \hline particle image diameter, $d_{t}$ & $1-2 p x$ \\
\hline magnification number, $M_{0}$ & 0.065 \\
\hline time step, $\Delta t$ & $600 \mu s$ \\
\hline focus depth, $\delta z$ & $9 m m$ \\
\hline light sheet thickness, $\Delta z$ & $2 m m$ \\
\hline numerical aperture, $f^{\#}$ & 4 \\
\hline interrogation area width, $D_{I A}$ & $32 p x$ \\
\hline interrogation area overlap & $50 \%$ \\
\hline camera resolution & $1280-1024 p x$ \\
\hline particle volume concentration, $C_{v}$ & $1.0 \cdot 10^{-4}$ \\
\hline
\end{tabular}

In the next sections certain aspects of the measurements are discussed based on a so-called reference case. The parameters for this reference case are $Q=Q_{d}, z / b=0.35$ (near the hub) and $\Omega=150 \mathrm{rpm}$.

\subsection{PIV measurement quality}

Before presenting all measured velocity fields, the quality of the PIV measurements is investigated. The measurements are analyzed here for the reference case $\left(Q=Q_{d}, z / b=\right.$ 0.35 and $\Omega=150 \mathrm{rpm}$ ), unless mentioned otherwise. Firstly, the PIV images and the corresponding vector fields are considered, followed by an investigation on the possible occurrence of peak-locking. Subsequently, the time averaging of instantaneous vector fields is studied. Finally, reproducibility is examined, by executing a second measurement at the default settings.

\subsubsection{PIV images}

A typical obtained PIV image of the considered impeller area is displayed in Fig. 5.14. Downstream of the trailing edge, the view is blurred by the presence of the diffuser screen. Clearly no reliable results can be expected here. Upstream of the leading edge (in the center of the right side of the images) the image is not sharp, due to the curvature of the hub (see also Fig. 5.11). No reliable results are expected here as well. Furthermore, the trailing edge casts a shadow in the image, which can be seen in the bottom right of the image. The blades can also be clearly seen in the picture, not only the hub part but also the shroud parts are visible. Due to reflections no good measurements can be expected here either. 


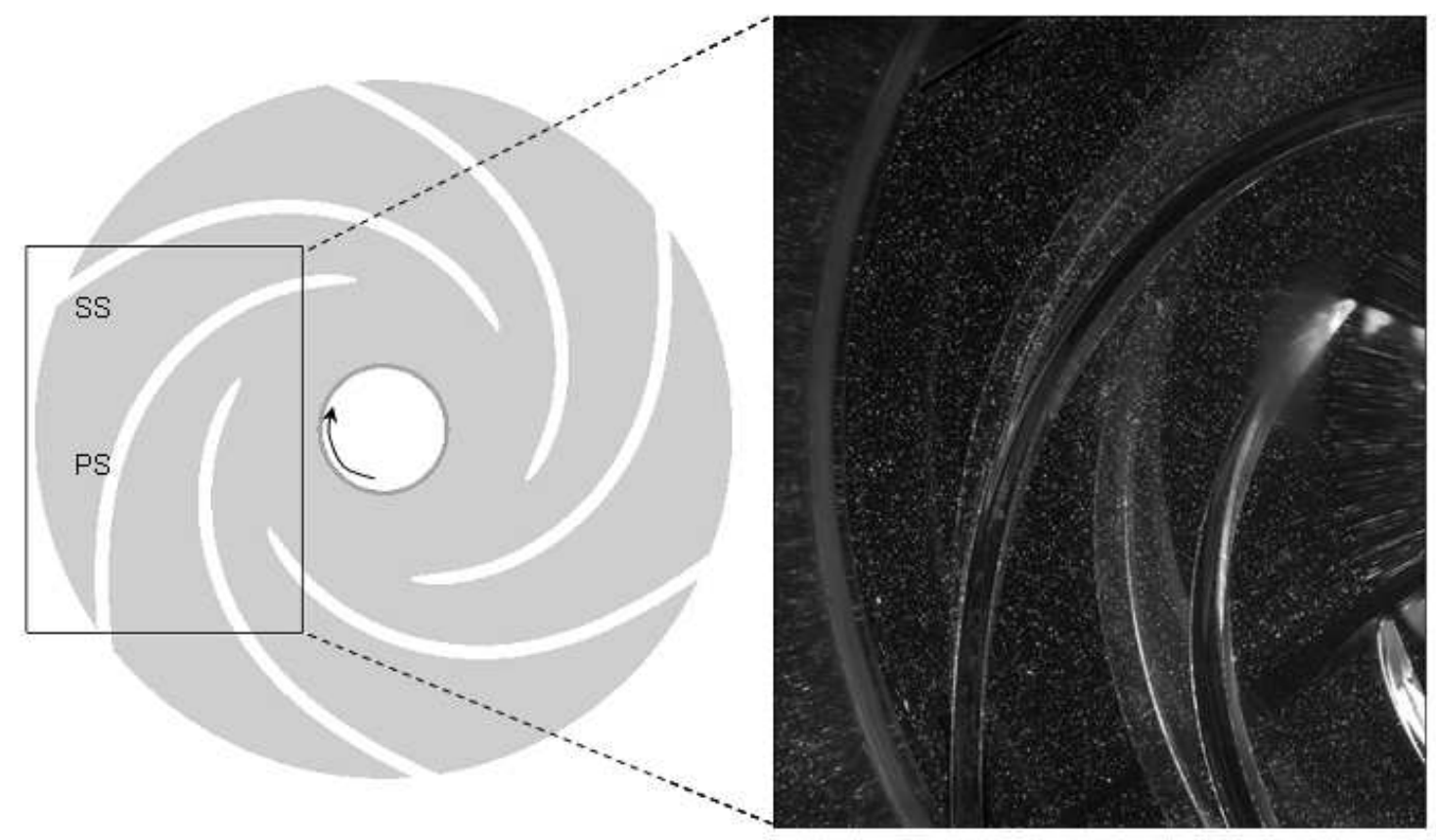

Figure 5.14: A typical PIV image of the considered impeller area.

At locations where no good results are expected, i.e. downstream of the trailing edge, upstream of the leading edge and at the blade locations, the relative velocity components are not considered and they are set to zero.

By analyzing an image pair (see Fig. 5.14 for one of the images) an instantaneous vector field of the absolute velocity $\boldsymbol{v}$ is determined using the PIV algorithm and the settings from table 5.1. Using the image calibration (see section 5.3.3), the relative velocity vector field $\boldsymbol{w}$ is determined from the absolute velocity vector field $\boldsymbol{v}$. To illustrate the influence of the post-processing parameters, one vector field is obtained for a single pass without median filter and one for multi-pass with median filter. The obtained results are compared in Fig. 5.15. First of all, it is concluded that the raw vector field of relative velocity is of acceptable quality for further post-processing, i.e. the number of spurious vectors does not exceed $5 \%$, which is a rule of thumb frequently used in PIV measurements. By applying post-processing options, the spurious vectors can be removed and replaced by interpolated values. For a more extensive investigation into the influence of post-processing options the reader is referred to $[2]$.

\subsubsection{Peak locking}

Peak locking or pixel locking occurs when the image size of the seeding particles is smaller than a single pixel. In that case a bias towards displacements of an integer number of pixels occurs, since no good sub-pixel interpolations can be performed (see section 5.3.2). In section 5.3.3 it has been noted that the predicted particle image diameter is slightly smaller than optimal, $d_{s}=0.95$. From the images that have been taken, however, it 

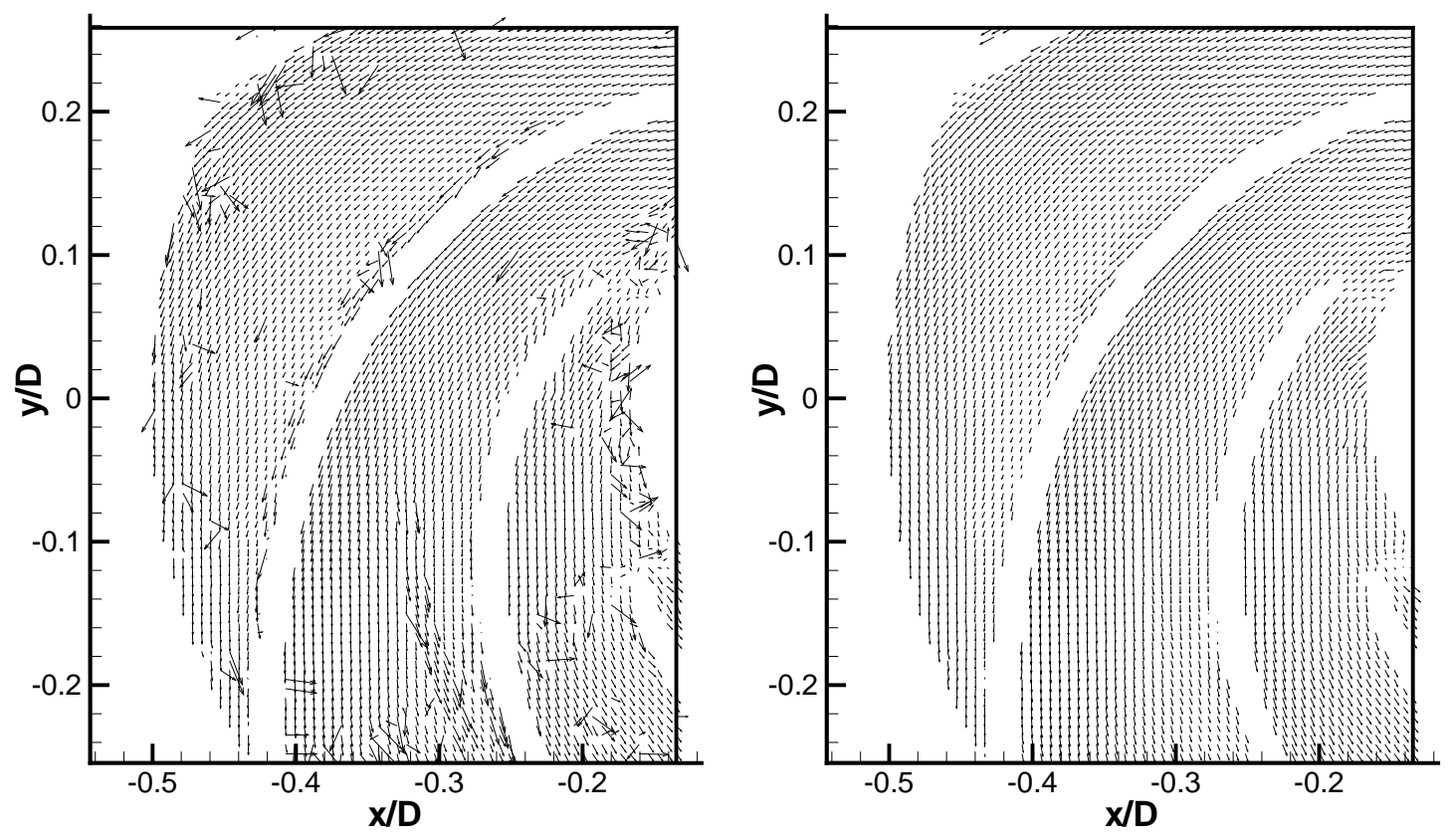

Figure 5.15: The influence of post-processing. An instantaneous relative velocity vector field for the reference case without (left) and with post-processing (right). The post-processing includes the use of a median filter and a multi-pass approach, i.e. a single pass for an interrogation area size of 64 by 64 pixels and two additional passes for an interrogation area size of 32 by 32 pixels.

appears that the particle image diameter is larger than the computed value, $1-2 p x$. Therefore, it is relevant to investigate the quality of the measurements with respect to peak locking.

In order to investigate this, the probability density function of all occurring displacements of an instantaneous vector field is studied. This is can be done for both the $x$ - and $y$-components of the displacement. The results are presented in Fig. 5.16 for an instantaneous measurement at the reference settings. It is observed that there are peaks in the probability density function. However, these peaks do not occur at integer values. Thus peak-locking does not occur.

To demonstrate the absence of peak locking effects more clearly, it is convenient to look at decimal values of the pixel displacements. For this purpose the following reduced displacements are defined

$$
\begin{gathered}
\Delta x^{*}=\Delta x-\text { floor }(\Delta x) \\
\Delta y^{*}=\Delta y-\text { floor }(\Delta y)
\end{gathered}
$$

where floor $(x)$ is a function which returns the largest integer value smaller than or equal to the value $x$. The probability density functions for $\Delta x^{*}$ and $\Delta y^{*}$ are shown in Fig. 5.17. There is practically no bias towards 0 or 1 , indicating that peak locking does not occur, 

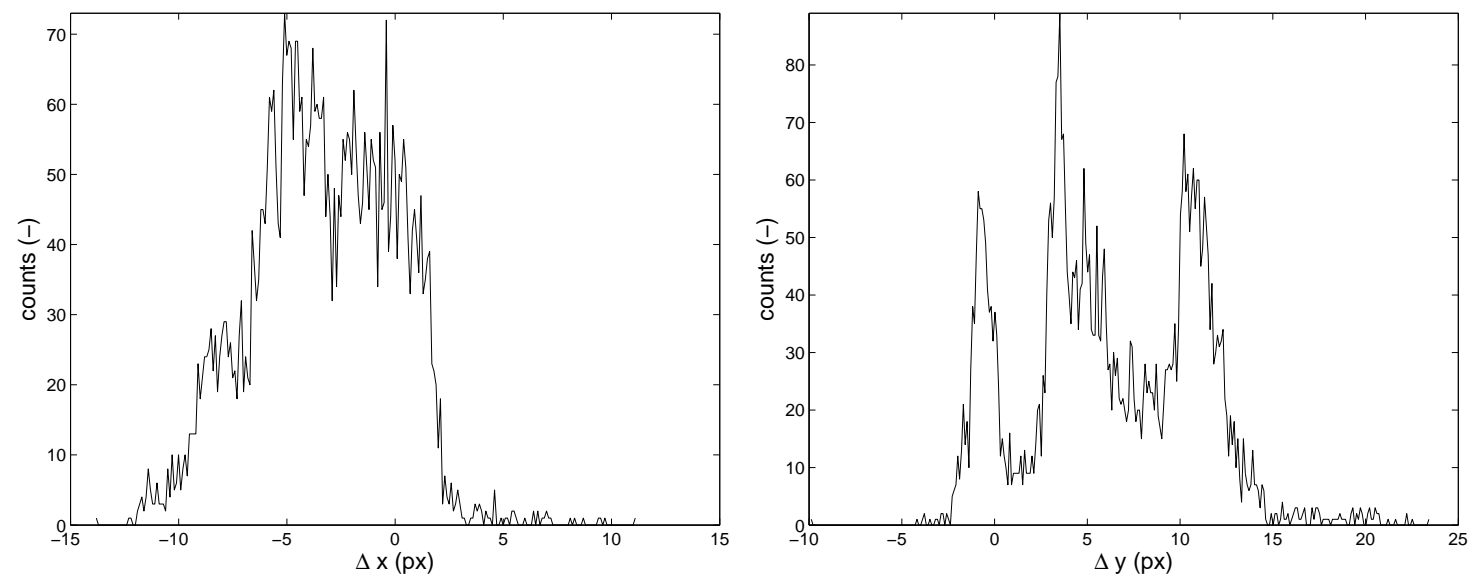

Figure 5.16: Probability density functions of particle displacements at default settings: the $x$-component of the displacement (left) and the $y$-component of the displacement (right).

and thus that the particle size is not too small, which is supported by analysis of the PIV images (see Fig. 5.13).
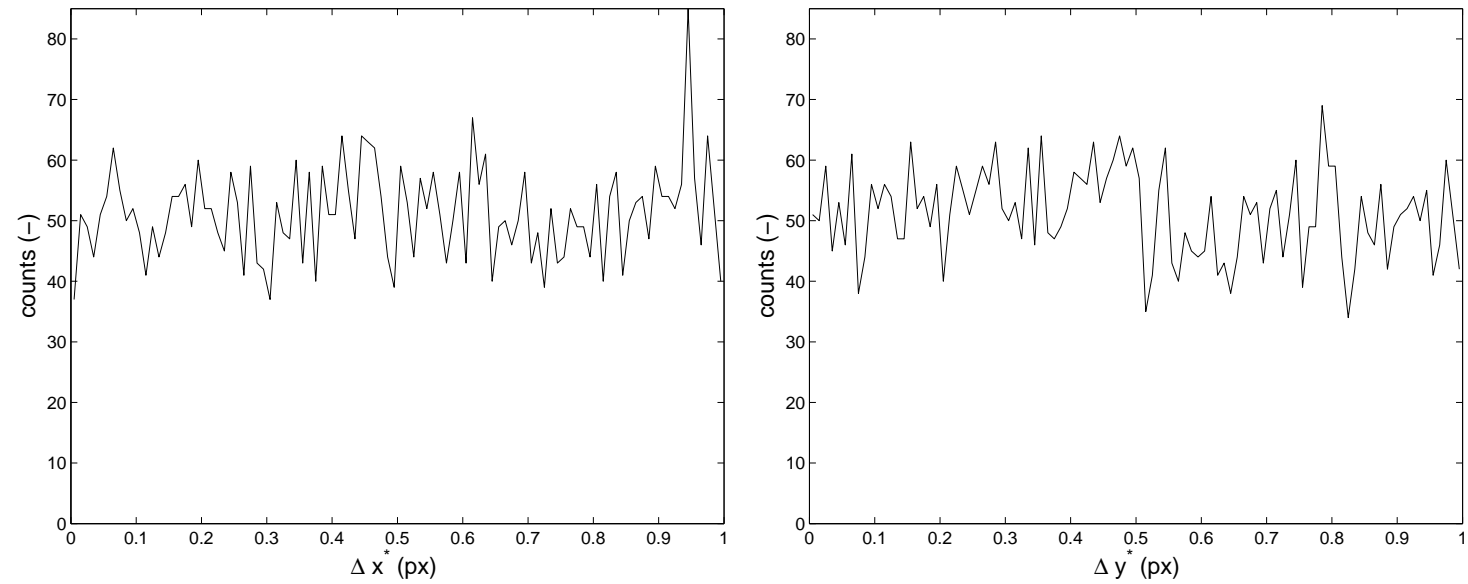

Figure 5.17: Probability density functions of reduced particle displacements at default settings. the $x$-component of the reduced displacement (left) and the $y$-component of the reduced displacement (right).

The reason that local maxima are present in the probability density function in Fig. 5.16, which is seen most clearly for the displacement in $y$-direction, is attributed to the fact that three different parts of an impeller channel are visible in the PIV image (see Fig. 5.14). The channel at the bottom right of the image corresponds to the lowest displacements in $y$-direction, the channel in the middle of the image to the middle peak in $y$-direction and the the channel in the top-left side of the image to the largest displacements in $y$-direction. Basically, the probability density function of each separate channel contributes to a part of the overall probability density function. 


\subsubsection{Time averaging}

In the flow model it is assumed that the flow field is stationary in the rotating frame, and also that the turbulence intensity $T u<<1$ (see section 2.1.1). Here we will investigate if these assumptions hold. The unbiased estimator of the standard deviation $\sigma$ of the velocity in the plane of measurement is defined here by

$$
\begin{gathered}
\sigma_{x}^{2}=\frac{1}{n-1} \sum_{i=1}^{n}\left(v_{x}^{i}-\bar{v}_{x}\right)^{2} \\
\sigma_{y}^{2}=\frac{1}{n-1} \sum_{i=1}^{n}\left(v_{y}^{i}-\bar{v}_{y}\right)^{2} \\
\sigma=\sqrt{\sigma_{x}^{2}+\sigma_{y}^{2}}
\end{gathered}
$$

where $n$ is the number of measurements, $v^{i}$ the instantaneous velocity and $\bar{v}$ the mean velocity. These root-mean square (rms) values are an indication for both the physical velocity fluctuations and the error in the measurements. In Fig. 5.18 the non-dimensional rms-values $\left(\sigma / u_{t e}\right.$, with $u_{t e}$ the blade speed at the trailing edge) are plotted for the default measurement in a contour plot for the whole domain and along a circular arc at $r / D=0.4$ from pressure to suction side. The circular arc is made up of two channels and its location is shown in Fig. B.1 in appendix B. Here $\theta^{*}$ is the distance from pressure to suction side along the circular arc, i.e. $\theta^{*}=0$ corresponds to the pressure side and $\theta^{*}=1$ to the suction side. Downstream of the impeller (large radii), upstream of the impeller (small radii) and at locations where the blades are located, the rms values are very high, indicating a low accuracy for the measurements there, as has been predicted previously (see section 5.4.1). These regions are therefore frequently masked out in the vector fields shown subsequently.
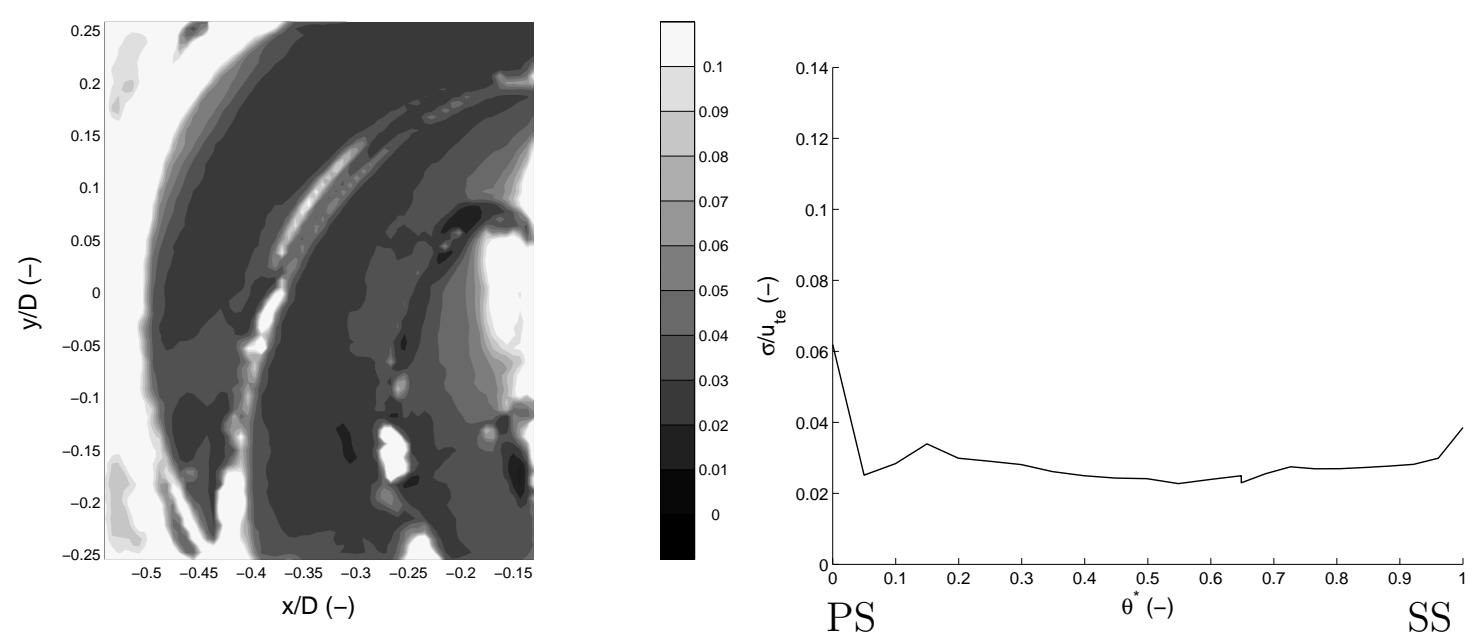

Figure 5.18: rms values $\sigma / u_{t e}$ for the default measurement. A contour plot (left) and the values from blade to blade along a circular arc at $r / D=0.4$ (right).

In pumps typically velocity fluctuations of $5 \%$ are encountered for the core flow [31]. The velocity fluctuations for the default measurement are investigated in more detail by 
considering the velocity fluctuations $v^{\prime}$ in a single point in the core of the flow, near the trailing edge. The results are plotted in Fig. 5.19. The velocity measurements are obtained at a frequency of $2.5 \mathrm{~Hz}(=150 \mathrm{rpm})$. The $\mathrm{rms}$ of the velocity fluctuations is found to be

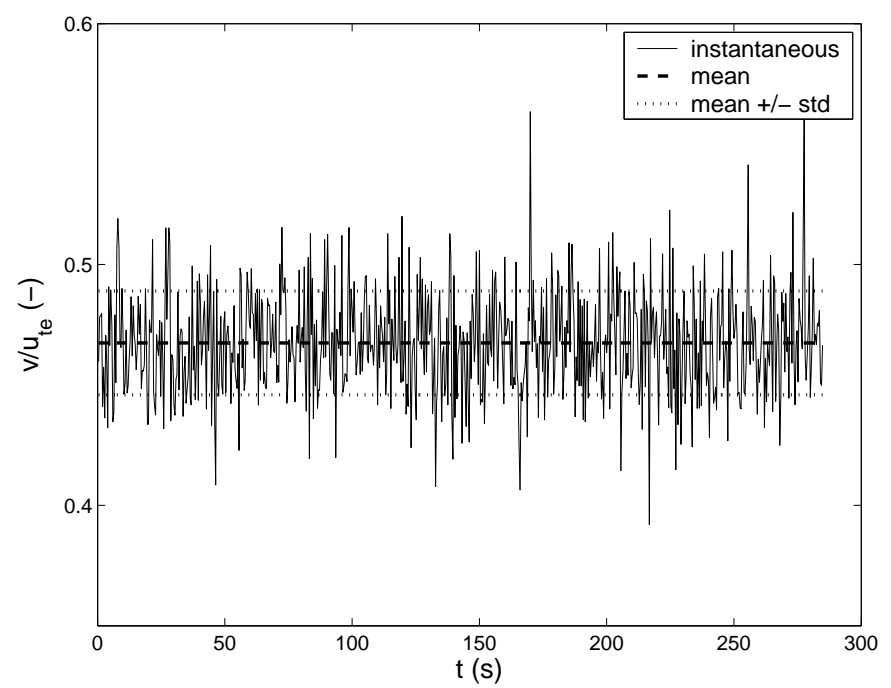

Figure 5.19: Velocity fluctuations of the size of the absolute velocity in the horizontal plane $v=\sqrt{v_{x}^{2}+v_{y}^{2}}$ at a point in the middle of a channel near the trailing edge for the default settings.

$4.6 \%$ for this measurement, which is of the same order of magnitude as observed elsewhere [31].

Concluding, the measured velocity fields can indeed be considered as stationary in the rotating frame of reference, with moderate velocity fluctuations.

\subsubsection{Reproducibility}

Following the series of measurements, reproducibility measurements were performed for the reference case, i.e. $Q=Q_{d}, z / b=0.35$ and $\Omega=150 \mathrm{rpm}$. To investigate whether differences occur, the original measurements at the reference settings are compared to these new measurements. The time-averaged velocity profiles for the original and the reproducibility measurement at $r / D=0.4$ are compared in Fig. 5.20. Clearly, the results are very similar indeed and the obtained profiles show adequate reproducibility. The time-averaged velocity profiles are generally within $1 \%$, except near the blades where the rms values are large (see the right image in Fig. 5.18)). 


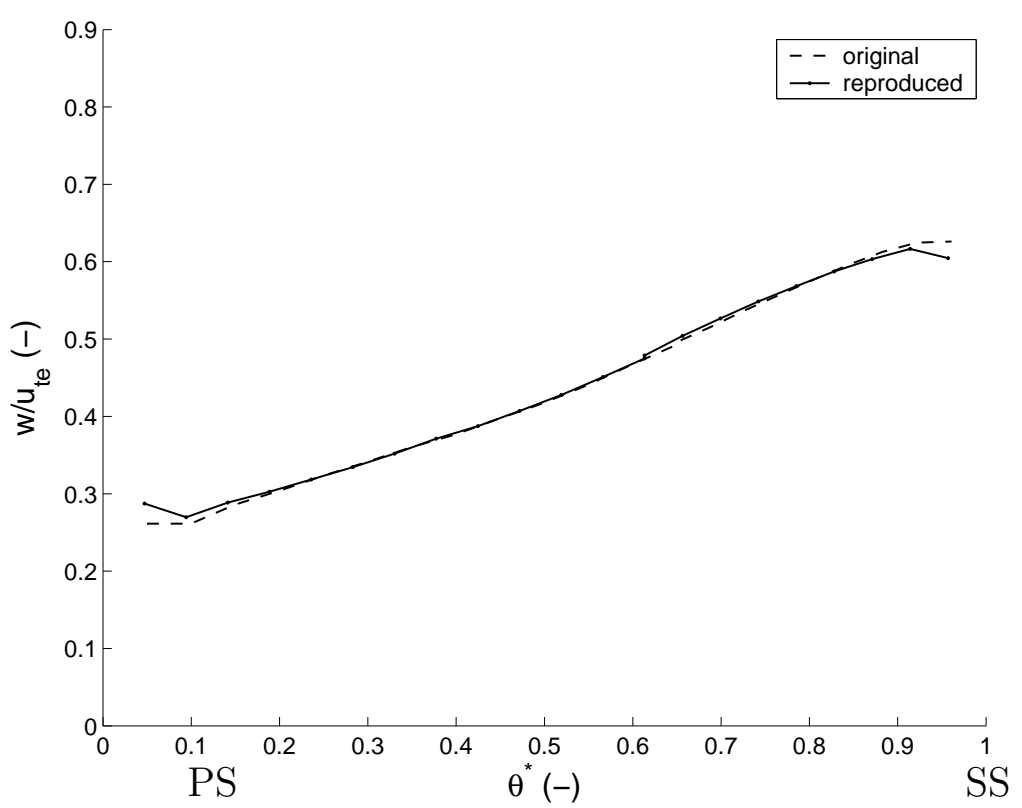

Figure 5.20: Comparison of relative velocity profiles obtained from the original and from the reproduced experiment along a circular arc at $r / D=0.4$ for the default settings $\left(Q=Q_{d}\right.$, $z / b=0.35$ and $\Omega=150 \mathrm{rpm}$ ). PS and SS indicate the pressure and suction side, respectively. 


\subsection{Measurement results for design conditions}

In this section the measurements at the design point are presented. For this purpose contour plots of the magnitude of the relative velocity, $w=\sqrt{w_{x}^{2}+w_{y}^{2}}$, in the measurement plane are shown. Streamlines are also plotted for one of the channels. The streamlines are generated from three starting points, located in the upper part of the plots at $y / D=0.24$. Furthermore, at a radius of $r / D=0.4$ the distributions of relative velocity are given from pressure to suction side along a circular arc in the measurement plane near hub and shroud. The measured relative velocity vector fields at design conditions near hub and shroud are also shown in Fig. B.1 and Fig. B.2 in appendix B. Here the circular arc, along which interpolated data are shown later, is depicted as well. The measurements performed at the design conditions are considered for $150 \mathrm{rpm}$, which corresponds to a Reynolds number of number of $R e=\left(\Omega D^{2}\right) /(2 \nu)=5.1 \cdot 10^{5}$.

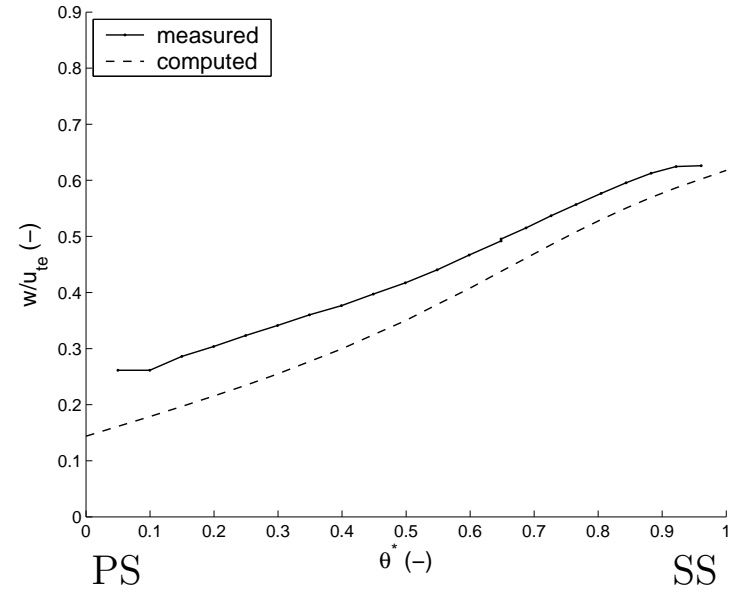

(a) near the hub, $z / b=0.35$

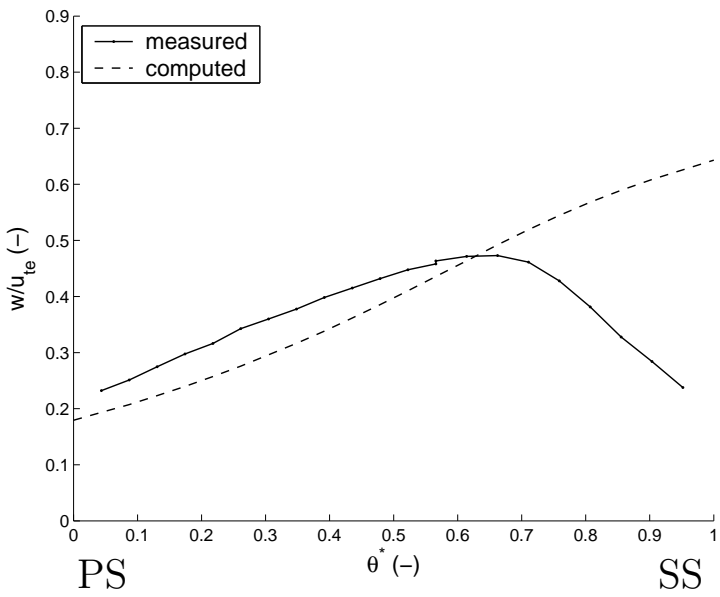

(b) near the shroud, $z / b=0.85$

Figure 5.21: Measured and computed profiles of relative velocity along a circular arc at $r / D=$ 0.4 for $Q=Q_{d}$. PS and SS indicate the pressure and suction side, respectively. The profiles consist of data fromx two impeller channels. The transitions is located at $\theta^{*}=0.64$ near the hub and at $\theta^{*}=0.57$ near the shroud.

In Fig. 5.22 the time-averaged relative velocity vector fields near the hub and shroud are compared with the computed results. In both measurements and computations a low relative velocity is seen, as expected, near the pressure side. Near the hub the relative velocity increases from pressure to suction side, corresponding to a drop in pressure.

This increase in relative velocity from pressure to suction side is shown even more clearly in Fig. 5.21a, where the velocity is interpolated to obtain the velocity along a circular arc of radius $r / D=0.4$. This circular arc covers two impeller channels (see Fig. B.1). Qualitatively, a similar profile is observed, only it is striking that near the hub the relative velocity is larger than that predicted by the computations. Note that at the blade surface, i.e. at the pressure side, $\theta^{*}=0$, and at the suction side, $\theta^{*}=1$, of the blade no measurement results are shown due to the unreliable results near the blade surface due to the reflections of the blade. 
In Fig. 5.22c and Fig. 5.22d the time-averaged relative velocity vector fields near the shroud are shown for measurements and computations, respectively. When studying these contour plots, it is observed that the relative velocity near the pressure side is low for both the measurements and the computations. However, in the plane near the shroud a region of low relative velocity is seen in the measurements near the suction side, which is not found in the computations. It appears that there is a kind of wake formation at the suction side, for larger radii. In this wake-like region the magnitude of relative velocity is low, but note that no back-flow occurs here, which is seen from the streamlines shown for the upper channel, or from the vector plots for the relative velocity shown in Fig. B.2. This wake-like region can also be considered as a thickened boundary layer. This phenomenon is not predicted by the computations, and is also not observed in the measurements performed in the plane near the hub. In Fig. 5.21b the velocity profile near the shroud is shown at a radius of $r / D=0.4$, and here also a drop in the magnitude of relative velocity in the plane near the suction side becomes apparent.

Due to the large drop in velocity near the shroud suction side, the average velocity in the plane near the shroud is lower than predicted by the computation. The presence of the suction side wake at the shroud leads to a blockage effect, which results in an increase in velocity in regions outside the wake, i.e. at the hub and also at the shroud pressure side, when compared with the computation.

The velocity profiles shown in Fig. 5.21 are composed of data from two separate impeller channels (see Fig. B.1) and the transition point between the channels is hard to spot in Fig. 5.21. Quantitatively, the difference in relative velocity for the transition point, the point where the data sets meet, is around $1 \%$, which falls within the measurement uncertainty. Therefore, it is concluded that the velocity profiles in all impeller channels are indeed identical, as was assumed in the derivation of the flow model presented in chapter 2.

Not only the magnitude of the relative velocity is of interest, but also its direction. To this end a flow angle $\beta_{w}$ is defined as

$$
\tan \beta_{w}=\frac{w_{r}}{w_{\theta}}
$$

This is the angle of the relative velocity vector with the circumferential direction. In Fig. 5.23 contour plots are shown for the flow angle for measurements and computations in the plane near hub and the one near the shroud. The flow angle distributions $\beta_{w}$ seen in experiment and computation are qualitatively comparable. For a large part of the considered domain, the flow angles are slightly smaller for the measurements, indicating that the relative flow is directed slightly more towards the pressure side. This is probably a result of the presence of the suction side wake area. Note that deviations resulting from the reflections of laser light at the blades near the shroud side are seen in the measurements (see also Fig. 5.14). 


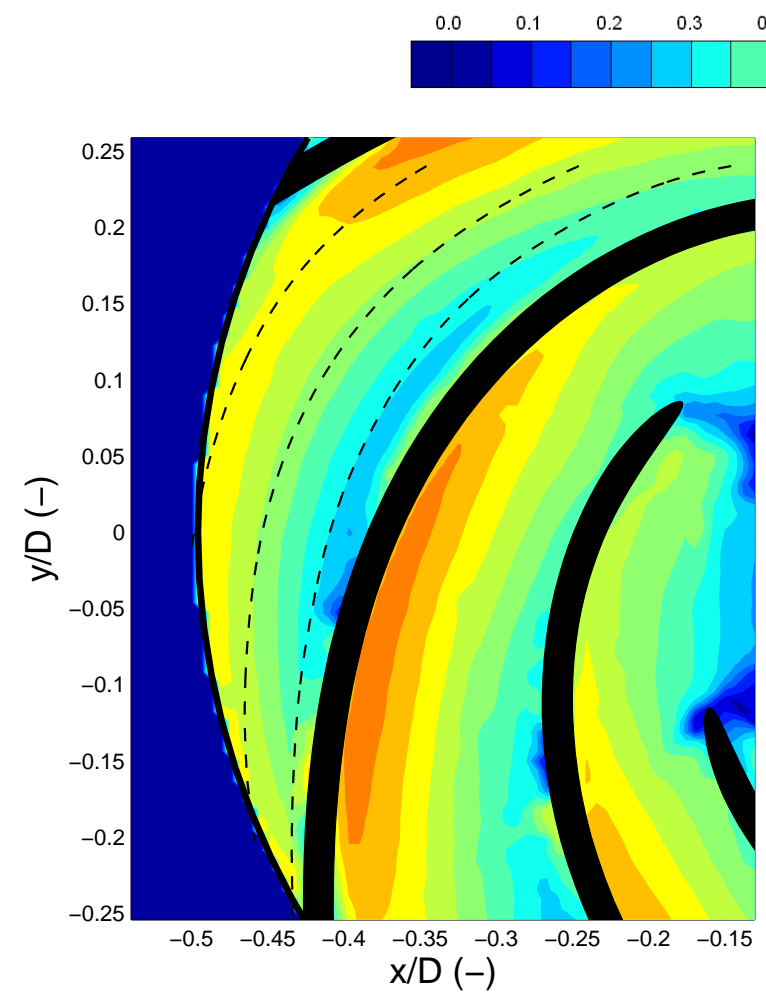

(a) measured flow field near hub

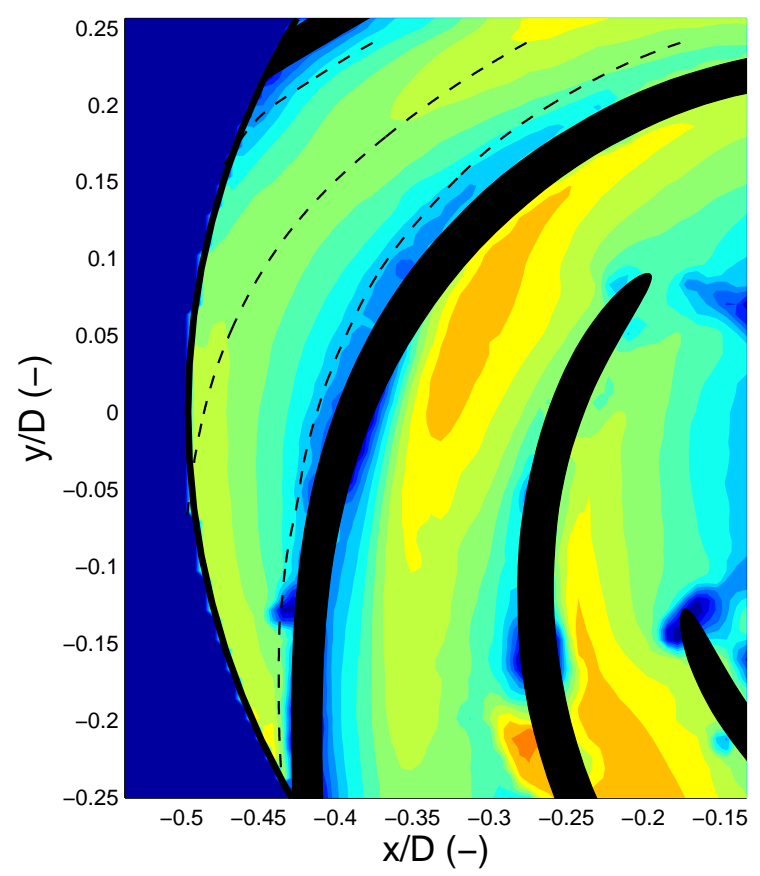

(c) measured flow field near shroud

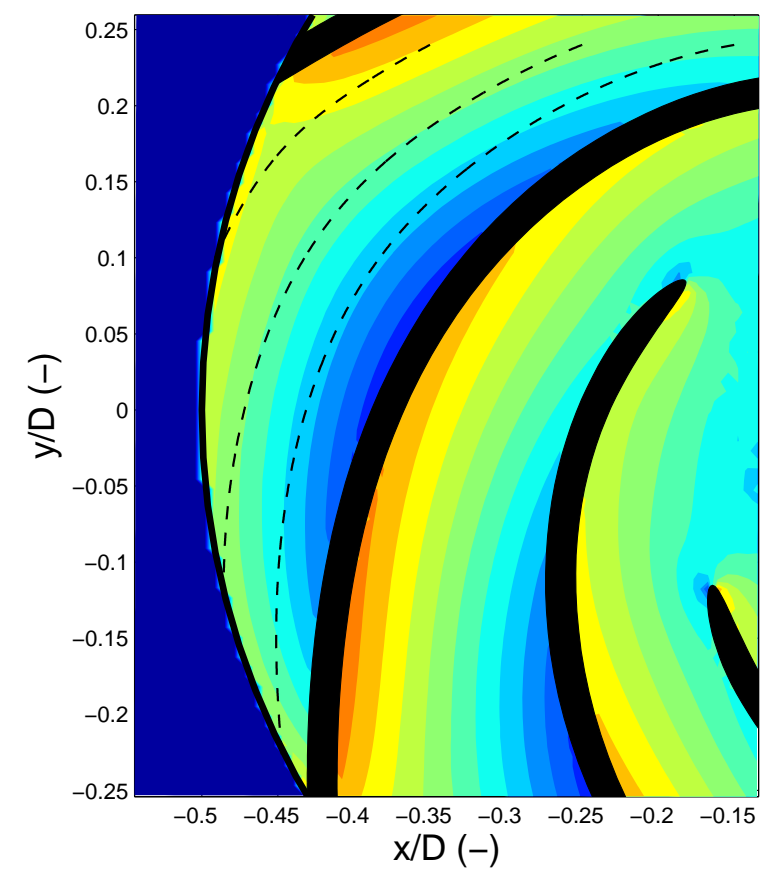

(b) computed flow field near hub

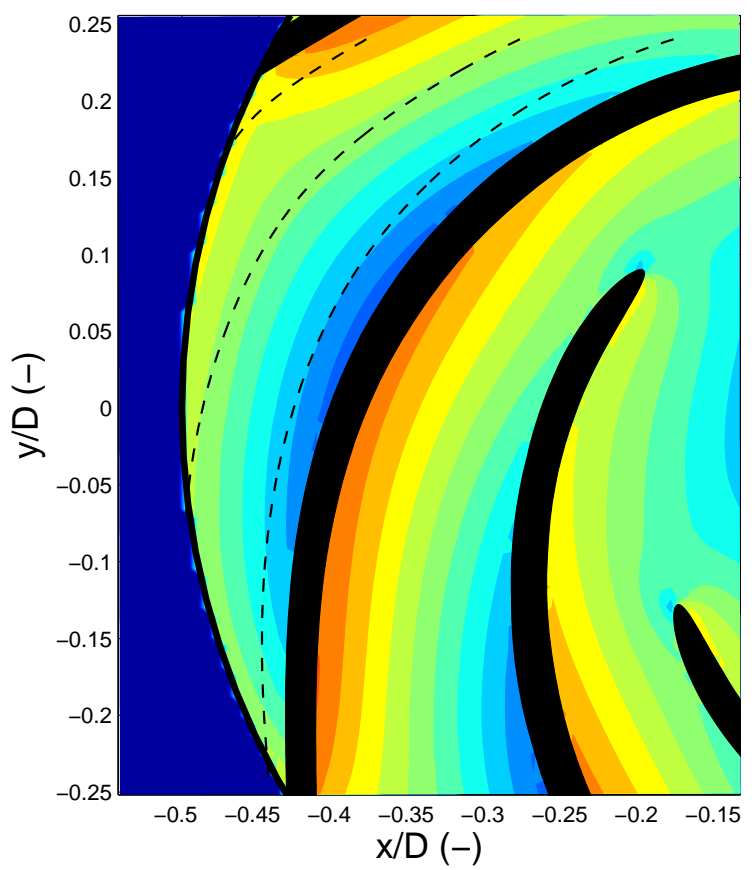

(d) computed flow field near shroud

Figure 5.22: Contour plots of measured and computed relative velocity, $w / u_{t e}$, near hub $(z / b=$ $0.35)$ and shroud $(z / b=0.85)$ at $Q=Q_{d}$. Streamlines, with starting points at $y / D=0.24$, are given for the upper channel with a dashed line. 


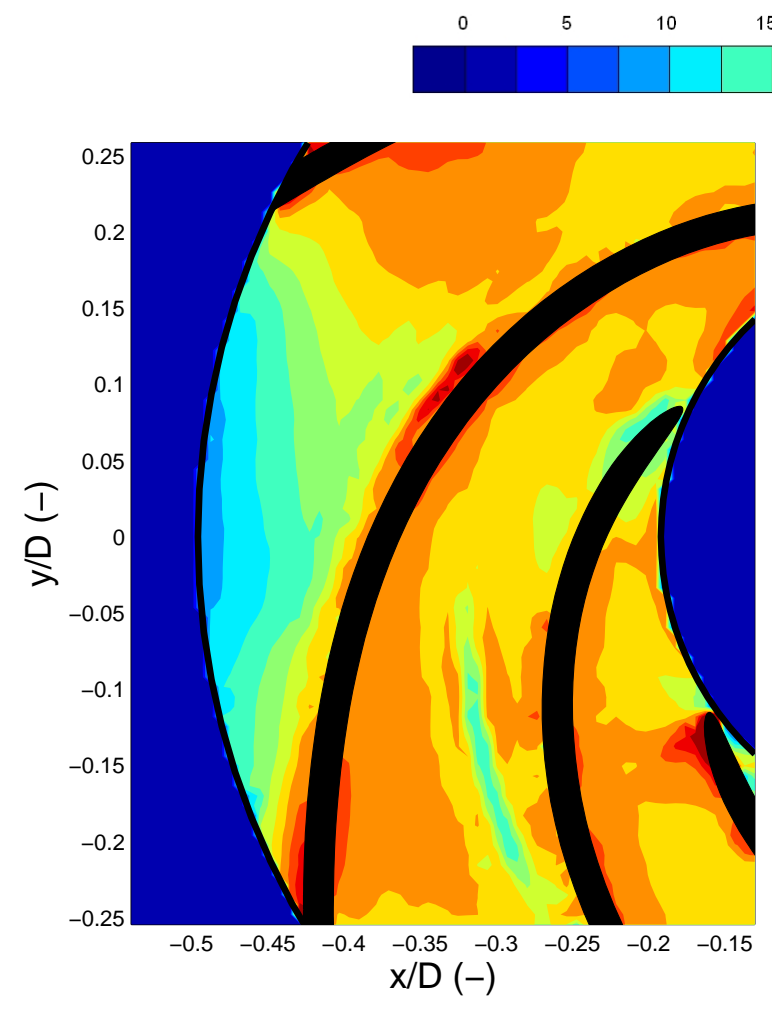

(a) measured flow angle near hub

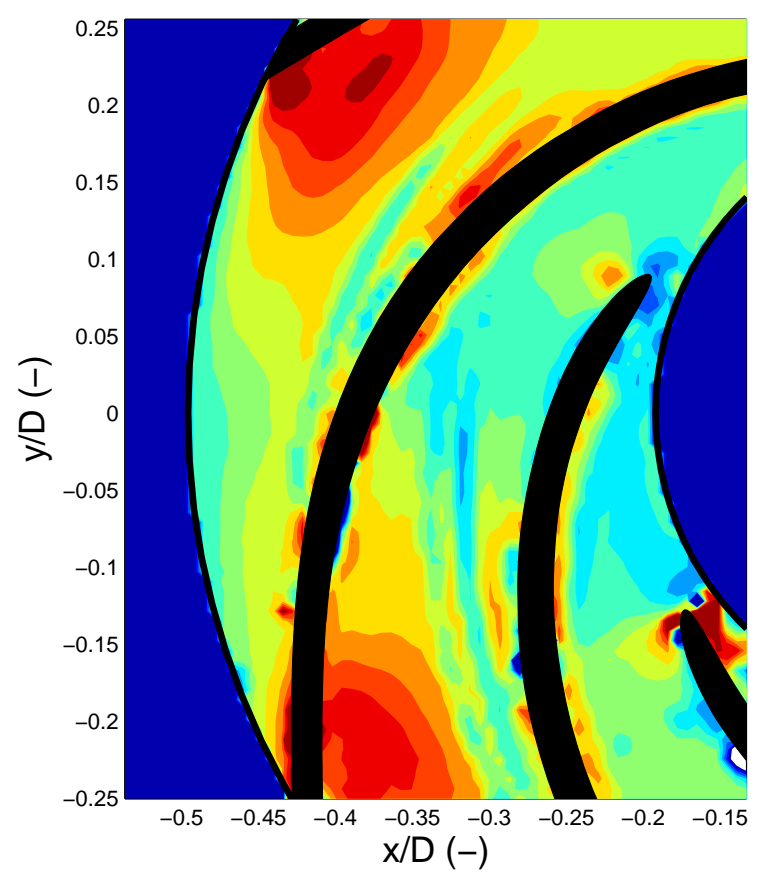

(c) measured flow angle near shroud

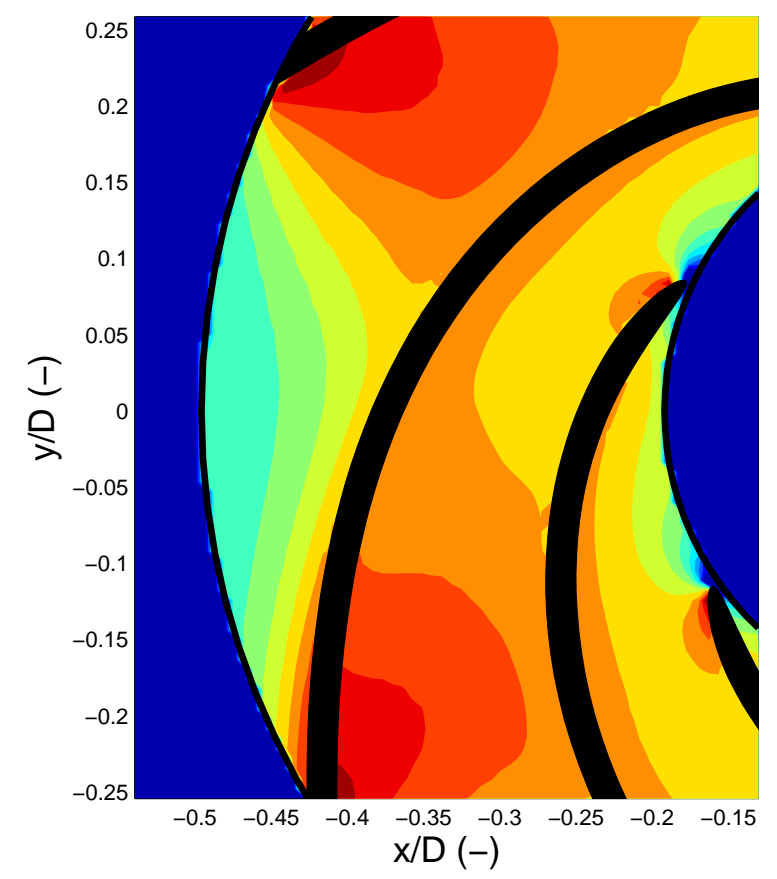

(b) computed flow angle near hub

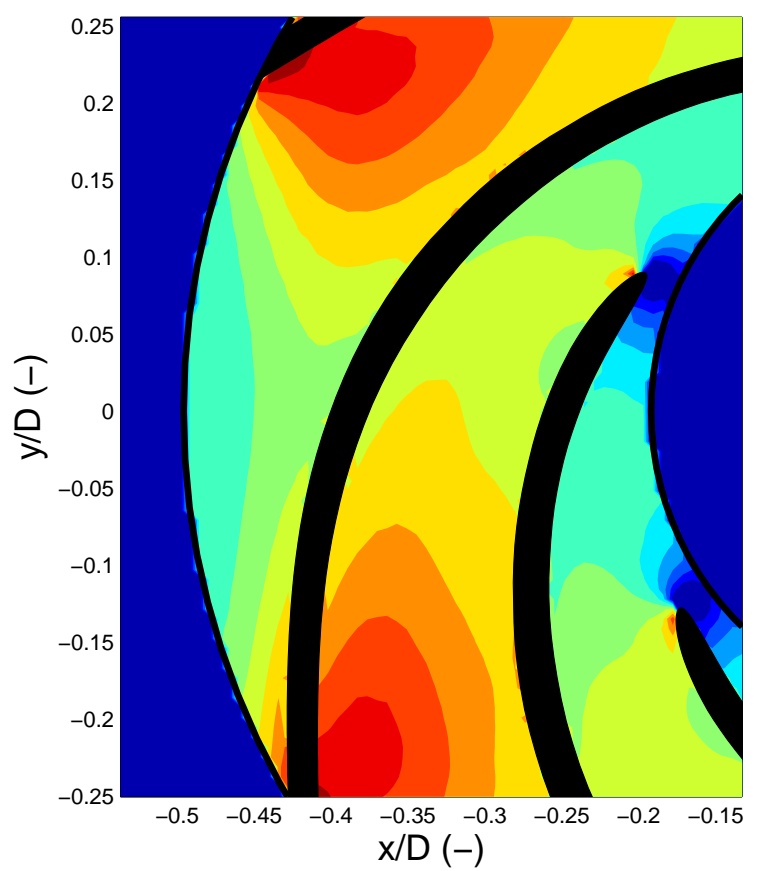

(d) computed flow angle near shroud

Figure 5.23: Contour plots of measured and computed flow angle $\beta_{w}$ in degrees near hub $(z / b=0.35)$ and shroud $(z / b=0.85)$ at $Q=Q_{d}$. 


\subsubsection{Influence of Reynolds number}

In the preceding section it has been shown that at 150rpm for design conditions a wake region of low relative velocity exists in the plane near the shroud, at the suction side. Before investigating the velocity profiles at off-design conditions, it is interesting to see if the Reynolds number has an effect on the resulting velocity fields. The wake region located at suction side is a region of low velocity and hence viscous effects are expected to be important. The appearance of this thickened boundary layer or wake can thus be affected by the Reynolds number. In the current setup it was not possible to increase the rotational speed above 200rpm (see section 5.2.2), therefore it has been chosen to lower the Reynolds number by a factor of 2 . This is achieved by lowering the rotational speed to $75 \mathrm{rpm}$ and changing the design flow rate $Q_{d}$ accordingly, leading to a Re-number of $2.6 \cdot 10^{5}$. The measurements are carried out in the plane near the shroud $(z / b=0.85)$, since this is the region where viscous flow effects are expected to be most important.

In Fig. 5.24 the contour plots for the (dimensionless) relative velocity are compared. The flow patterns are rather similar indeed, as is also seen from the velocity distribution at $r / D=0.4$ displayed in Fig. 5.25. Note that the dimensionless velocity profiles obtained from the computations are independent of the Reynolds number, since viscous effects are not considered in the potential flow model. When examining these figures more closely, it appears that the wake area is slightly larger for the measurements performed at $75 \mathrm{rpm}$. However, the effect is rather small. Therefore, it is advised to carry out similar investigations at rotational speeds at 300 and $600 \mathrm{rpm}$ in the future, to investigate whether this wake area decreases in size for increasing Reynolds numbers. Currently, these rotational speeds could not be realized with the experimental setup (see section $5.2 .2)$. 


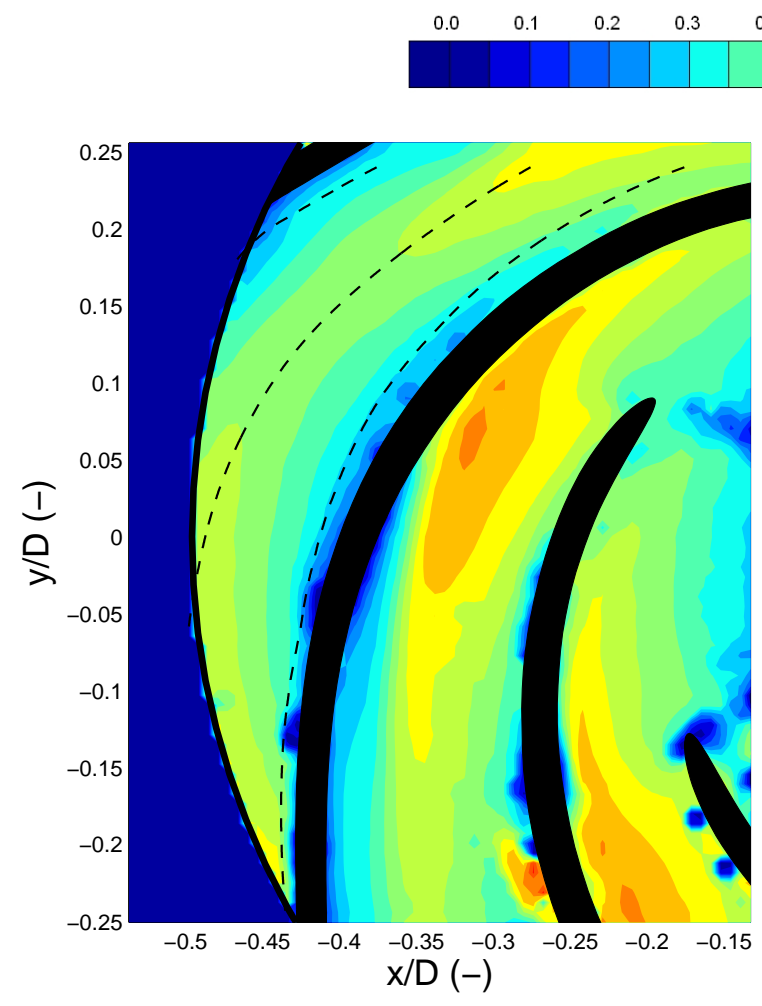

(a) $75 \mathrm{rpm}$

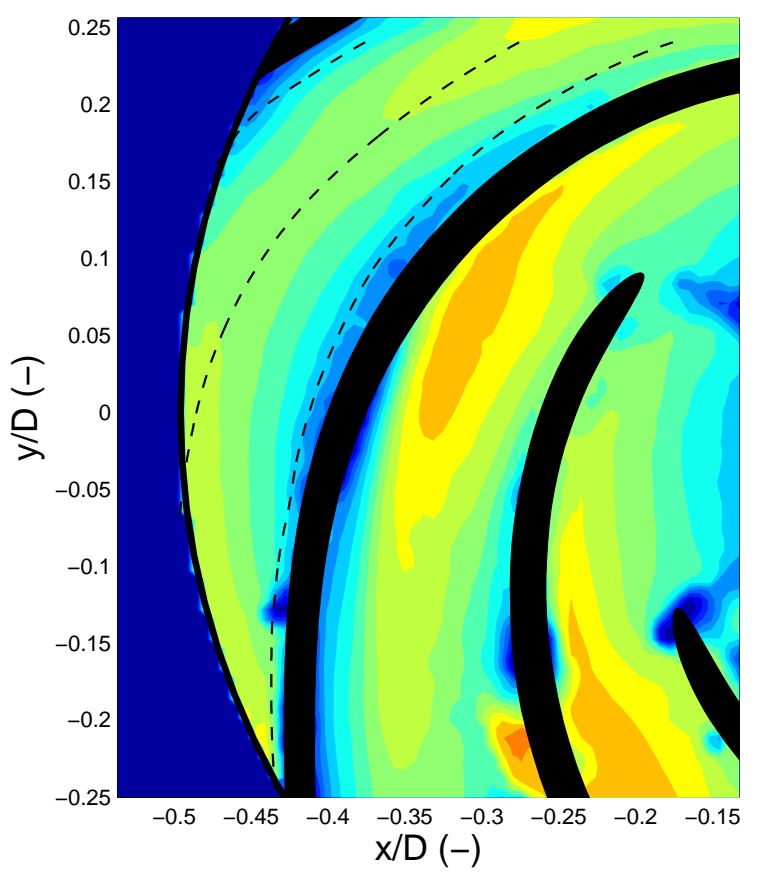

(b) $150 \mathrm{rpm}$

Figure 5.24: Contour plots of measured relative velocity $w / u_{t e}$ at $Q=Q_{d}$. A comparison between 75 and $150 \mathrm{rpm}$ near the shroud $(z / b=0.85)$. Streamlines, with starting points at $y / D=0.24$, are given for the upper channel with a dashed line.

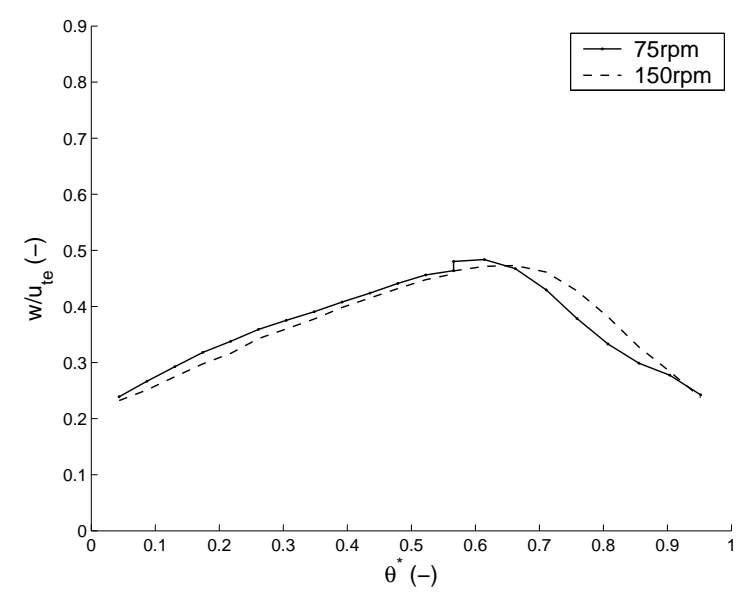

Figure 5.25: Measured profiles of relative velocity along a circular arc at $r / D=0.4$ for $Q=Q_{d}$ near the shroud $(z / b=0.85)$. A comparison between the results at 75 and $150 \mathrm{rpm}$. The measured profiles are composed of data from two impeller channels, the transition is at $\theta^{*}=0.57$. 


\subsection{Measurement results at off-design conditions}

In this section measurement results at off-design conditions are presented. The measurements have been performed at a rotational speed of $150 \mathrm{rpm}$ for flow rates in a range of $0.5 Q_{d}<Q<1.5 Q_{d}$. The lower flow rates are discussed in sections 5.6.1 and 5.6.2. The higher flow rates in sections 5.6.3 and 5.6.4.

\subsection{1 $Q=0.8 Q_{d}$}

The first off-design flow rate that is considered is $Q=0.8 Q_{d}$. The computations carried out for this flow rate have been included in the optimization of the impeller (see section 4.3). The time-averaged results are presented in Fig. 5.27 in the plane near the hub and the shroud. Compared to the results at the design point, the results obtained at $Q=0.8 Q_{d}$ show similar features. The measured velocity in the plane near the hub is larger than predicted by the computations, which is best seen in Fig. 5.26a. For this flow rate, a small wake can be seen near the trailing edge at the hub side (see Fig. 5.27a), indicating that for lower flow rates suction side wakes also occur in the plane near the hub. Note that

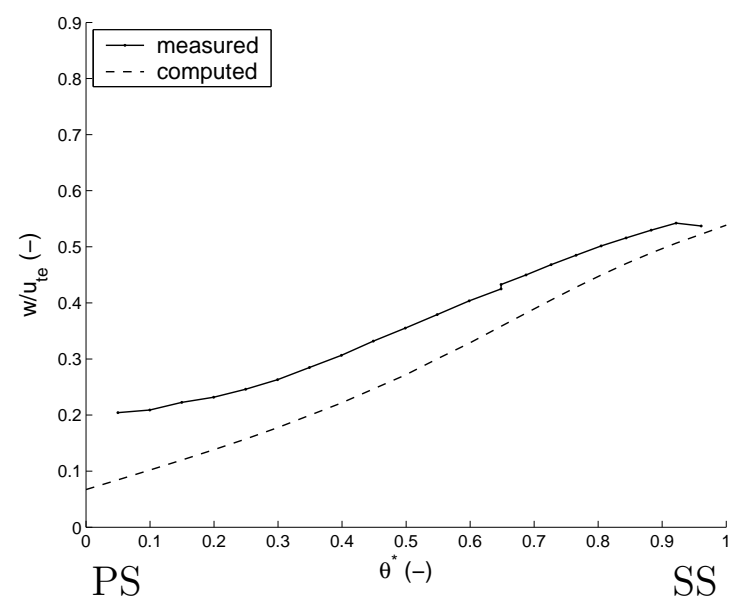

(a) near the hub, $z / b=0.35$

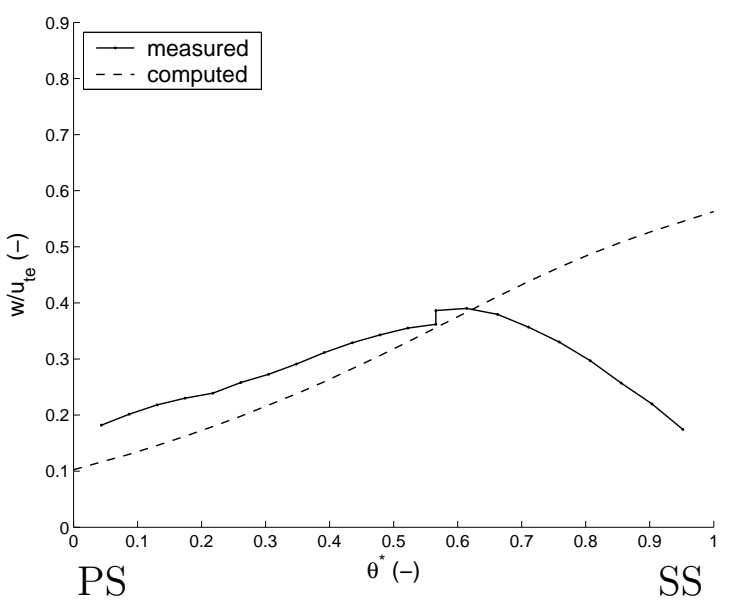

(b) near the shroud, $z / b=0.85$

Figure 5.26: Measured and computed profiles of relative velocity along a circular arc at $r / D=$ 0.4 for $Q=0.8 Q_{d}$. The profiles consist of data from two impeller channels, the transition is at $\theta^{*}=0.64$ for the hub and at $\theta^{*}=0.57$ for the shroud.

the matching of the two data sets in the plane at the shroud is more apparent than for the design conditions (5\% difference, see Fig. 5.26b), indicating that the difference in flow velocity between the two channels exceeds the frequently encountered values of around $1 \%$. 


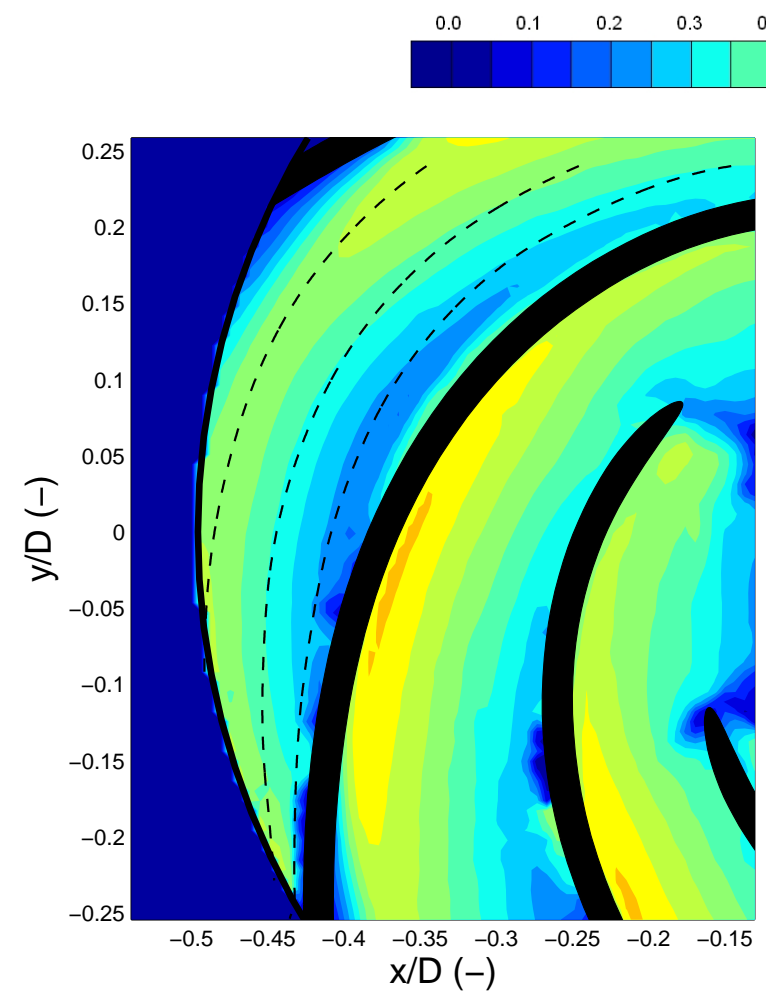

(a) measured flow field near hub

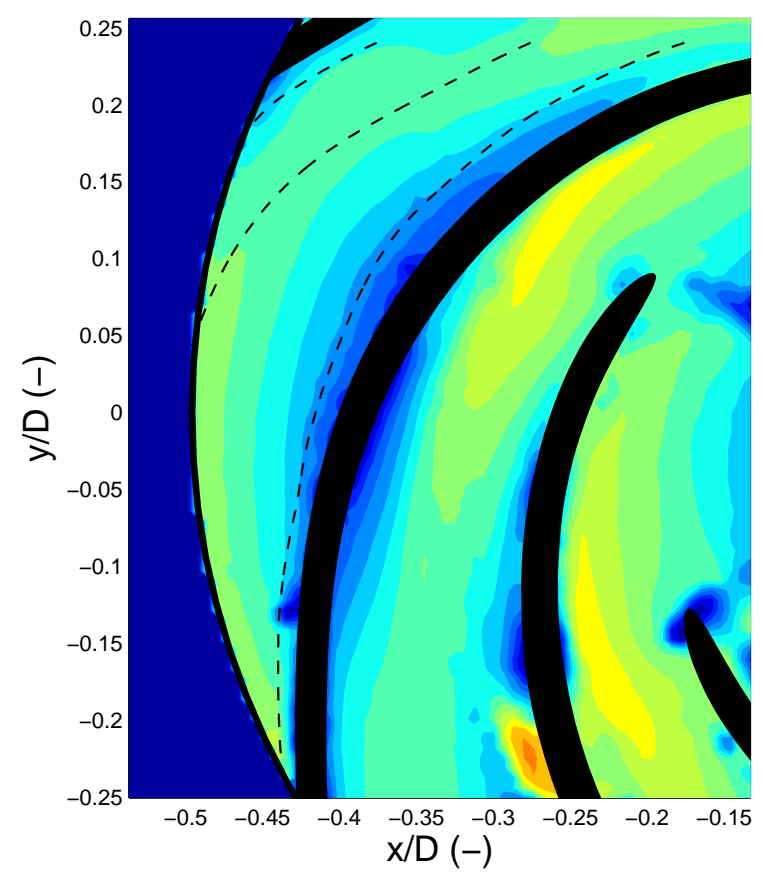

(c) measured flow field near shroud

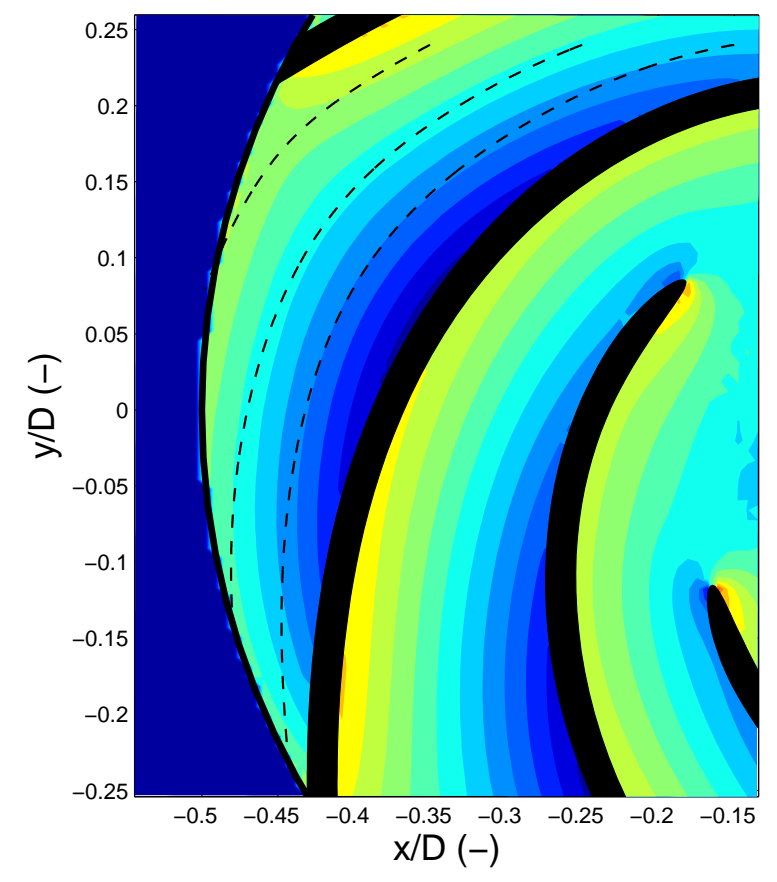

(b) computed flow field near hub

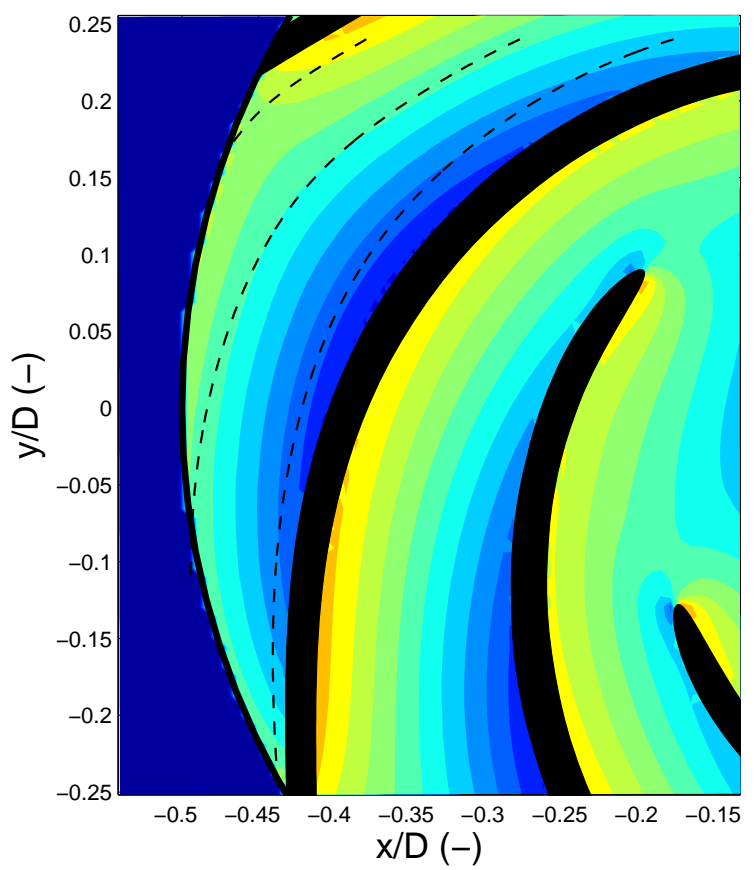

(d) computed flow field near shroud

Figure 5.27: Contour plots of measured and computed relative velocity, $w / u_{t e}$, near hub $(z / b=0.35)$ and shroud $(z / b=0.85)$ at $Q=0.8 Q_{d}$. Streamlines, with starting points at $y / D=0.24$, are given for the upper channel with a dashed line. 


\subsection{2 $Q=0.5 Q_{d}$}

The second off-design flow rate that is considered is that of $Q=0.5 Q_{d}$. This is already far off-design and the predictions from the potential flow model are expected to deteriorate considerably, and this is indeed the case. The measured and computed velocities in both measurement planes are shown in Fig. 5.29. Moreover, the distribution of relative velocity magnitude is shown at $r / D=0.4$ near hub and shroud in Fig. 5.28. For this low flow rate, it can clearly be seen that not only at the shroud, but also at the hub a wake region exists at the suction side of the blade. The existence of low velocities near the suction and pressure side leads to a flow pattern which in a way resembles a jet: high velocities in the center of the channel and low velocities near the surfaces of the blade. The low velocity region at the suction side results in a blockage effect. This in turn causes the velocities at the pressure side to be higher than predicted by the inviscid flow model. Therefore, no back-flow region near the pressure side is observed for the measurements, whereas a small back-flow region at the hub is predicted by the potential flow model, caused by the counter vortex.

For this flow rate the measured vector fields of relative velocity are shown in appendix B in Figs. B.3 and B.4 for the plane near the hub and the shroud, respectively. From these figures it can be seen that the observed suction-side wakes near hub and shroud are regions of low velocity. However, note that flow-reversal does not occur.

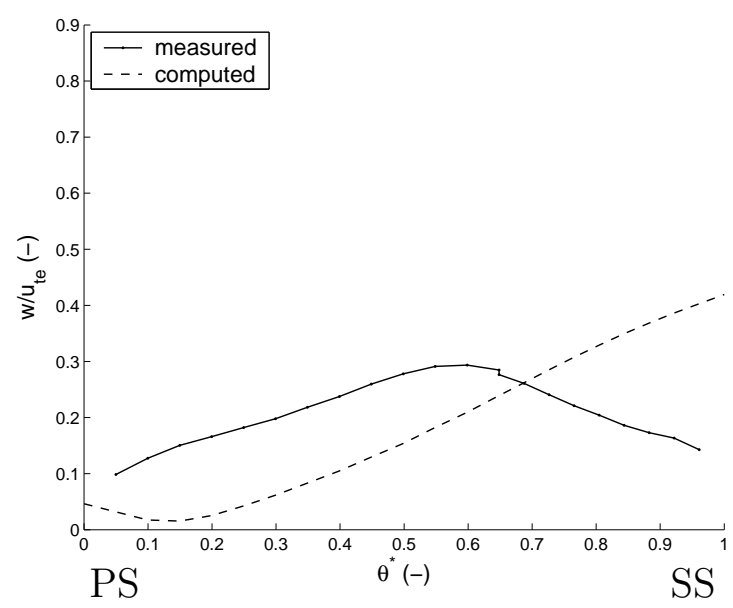

(a) near the hub, $z / b=0.35$

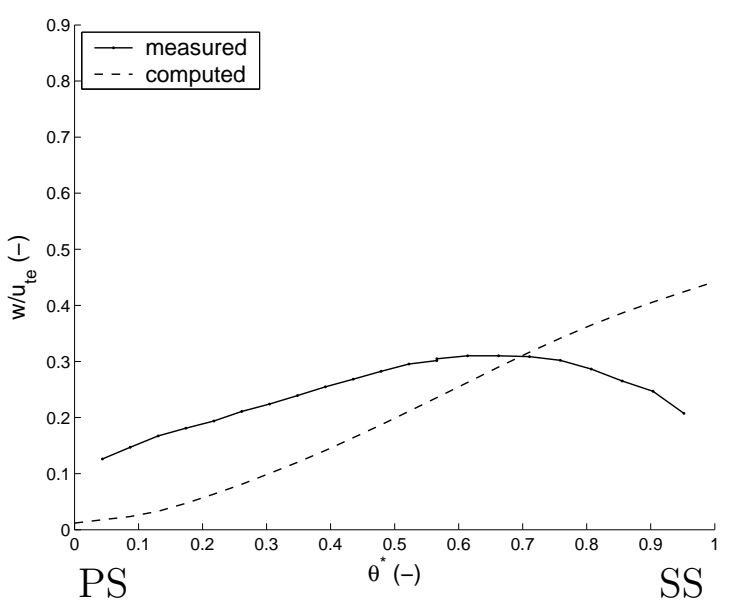

(b) near the shroud, $z / b=0.85$

Figure 5.28: Measured and computed profiles of relative velocity along a circular arc at $r / D=$ 0.4 for for $Q=0.5 Q_{d}$. The profiles consist of data from two impeller channels, the transition point is at $\theta^{*}=0.64$ for the hub and at $\theta^{*}=0.57$ for the shroud. 


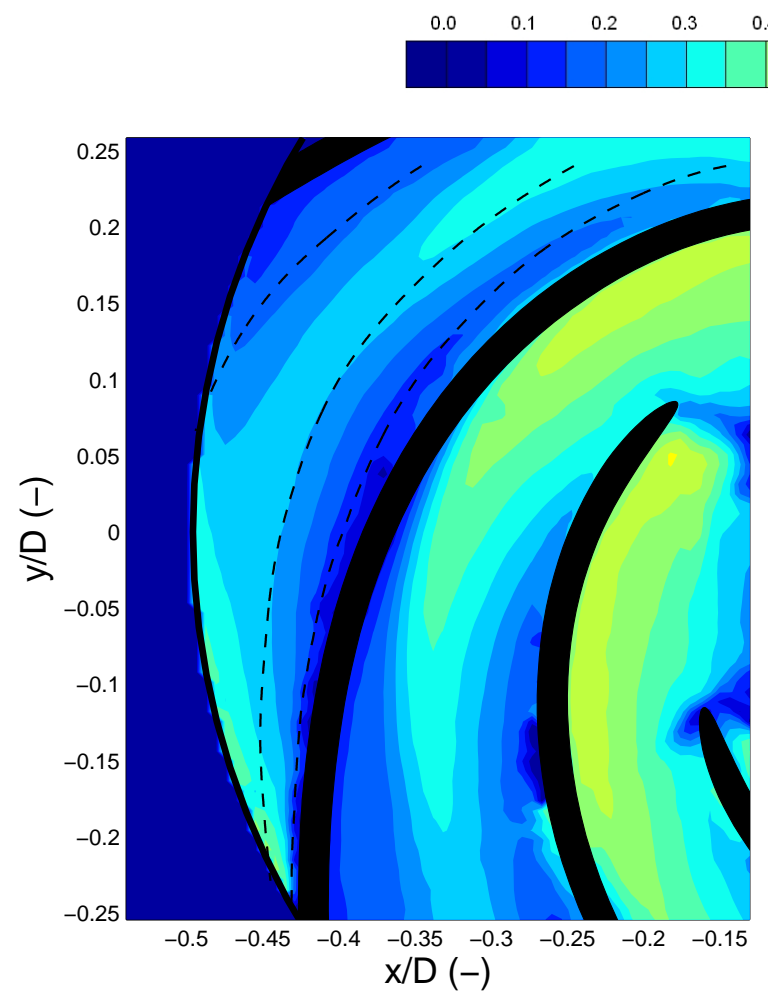

(a) measured flow field near hub

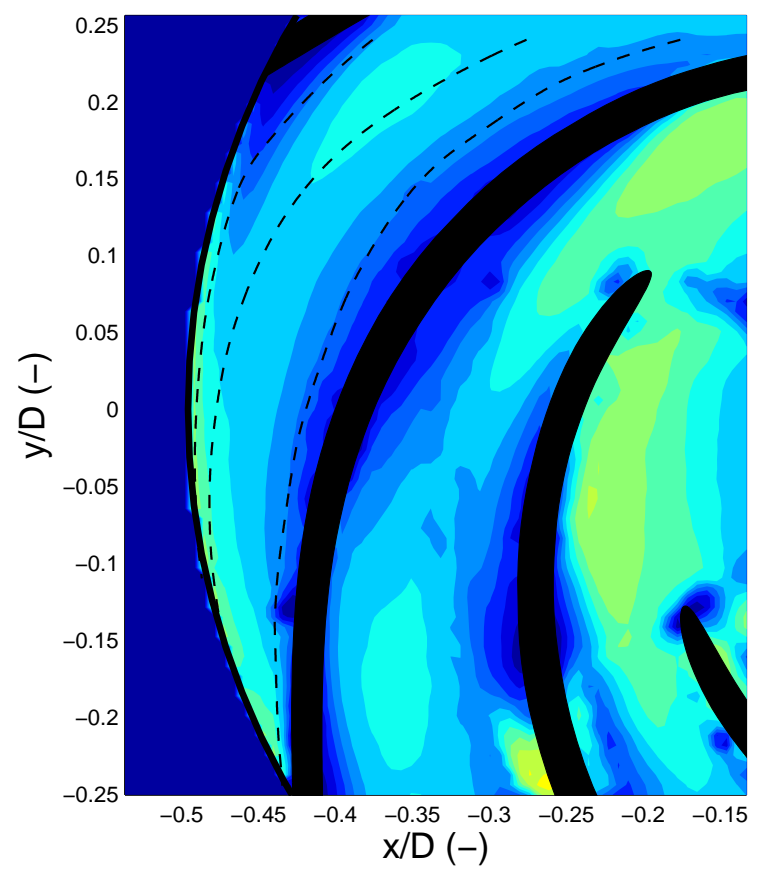

(c) measured flow field near shroud

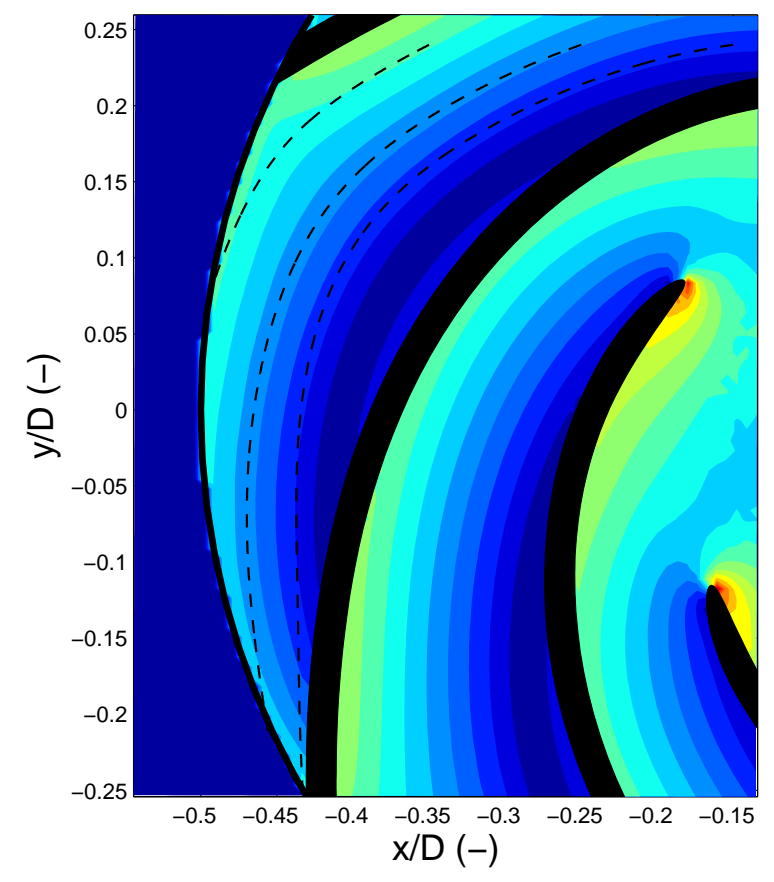

(b) computed flow field near hub

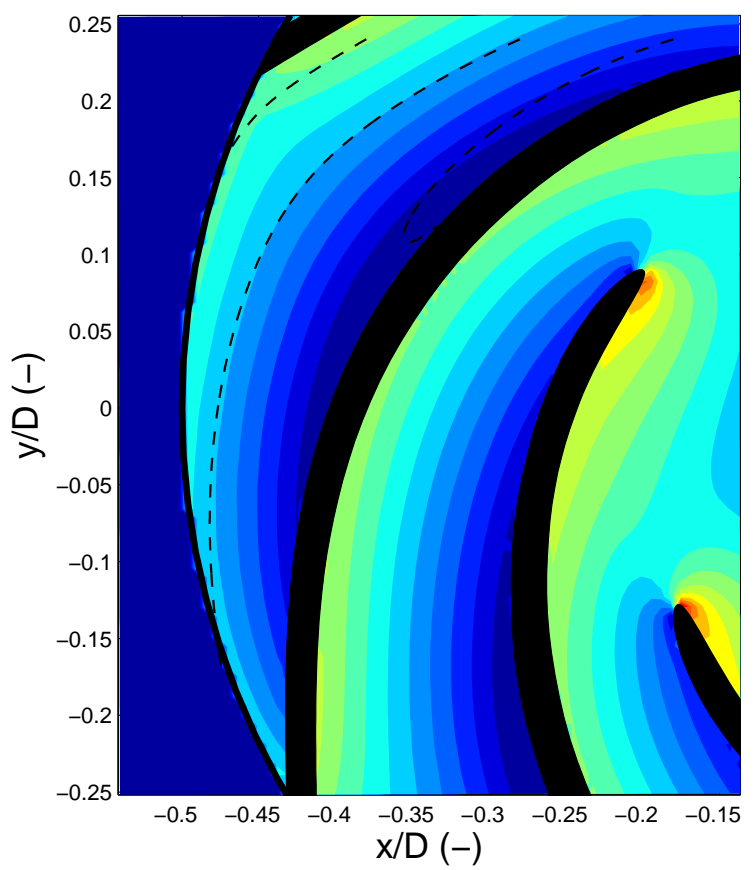

(d) computed flow field near shroud

Figure 5.29: Contour plots of measured and computed relative velocity, $w / u_{t e}$, near hub $(z / b=0.35)$ and shroud $(z / b=0.85)$ at $Q=0.5 Q_{d}$. Streamlines, with starting points at $y / D=0.24$, are given for the upper channel with a dashed line. 


\subsection{3 $Q=1.2 Q_{d}$}

Measurements have also been performed at a flow rate of $Q=1.2 Q_{d}$, which is one of the flow rates at which the computed results are used in the optimization (see section 4.3). For this high flow rate the measured and computed distributions of relative velocity are shown in the measurement plane near the hub and the shroud in Fig. 5.31. To show the differences between the measurements and computations more clearly, the velocity profiles at a radius of $r / D=0.4$ are shown in Fig. 5.30. From these results it can be concluded that the situation is very much comparable to that at the design point, $Q=Q_{d}$. In the plane close to the hub larger velocities are measured than predicted, and no wake exists at the suction side. In the plane near the shroud, a wake region is present at the suction side. This wake is somewhat smaller than for the design flow rate.

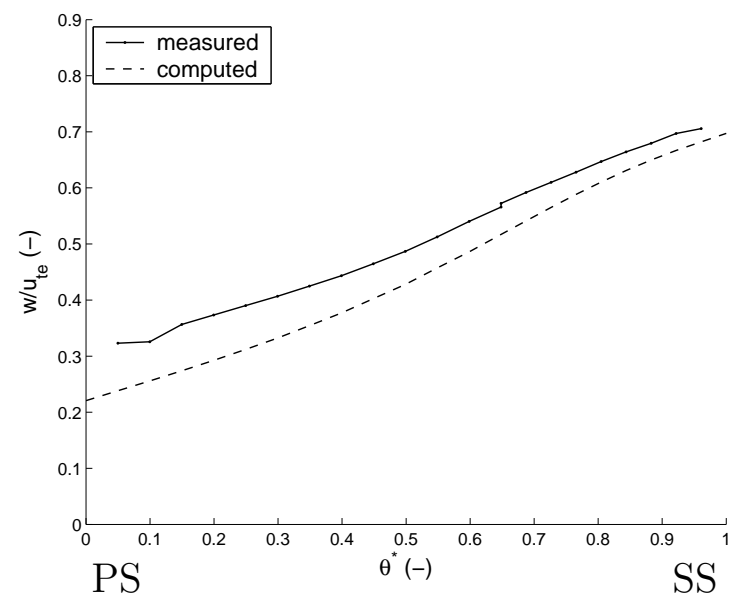

(a) near the hub, $z / b=0.35$

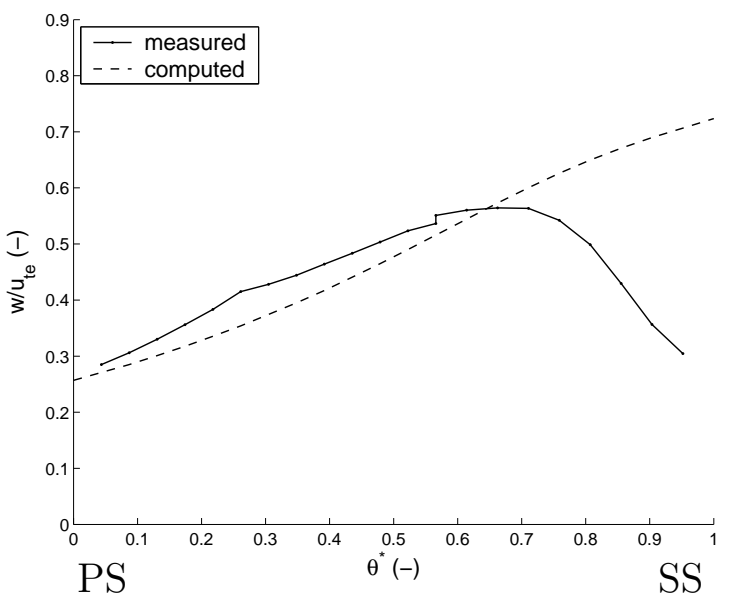

(b) near the shroud, $z / b=0.85$

Figure 5.30: Measured and computed profiles of relative velocity along a circular arc at $r / D=$ 0.4 for $Q=1.2 Q_{d}$. The profiles consist of data from two impeller channels, the transition is at $\theta^{*}=0.64$ for the hub and at $\theta^{*}=0.57$ for the shroud. 


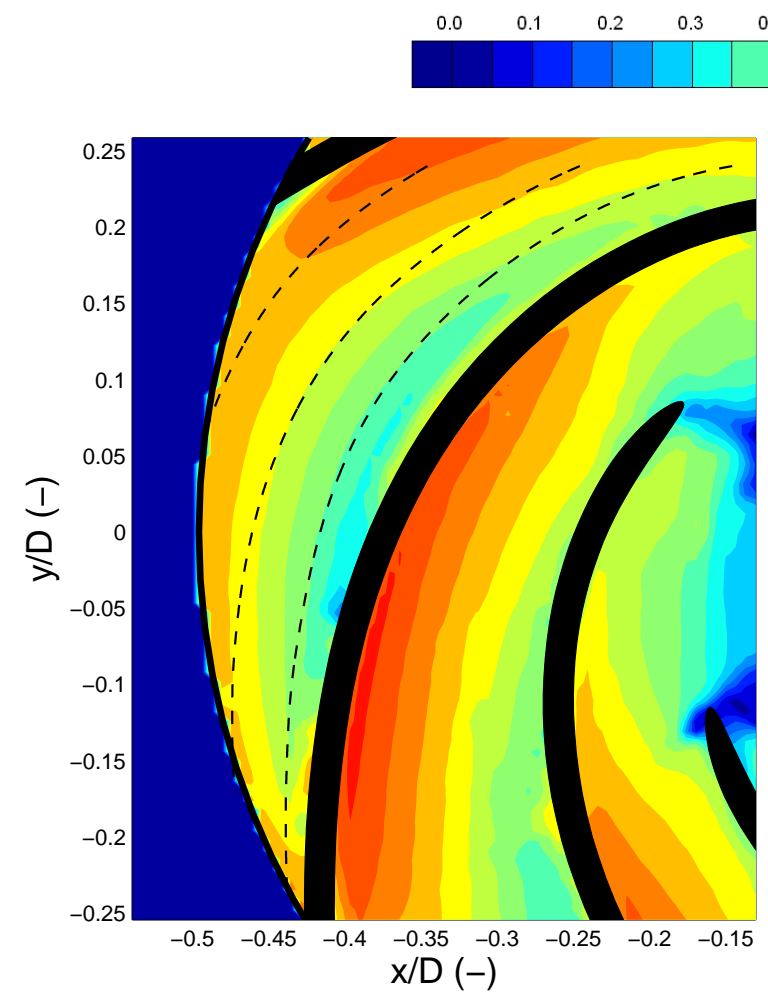

(a) measured flow field near hub

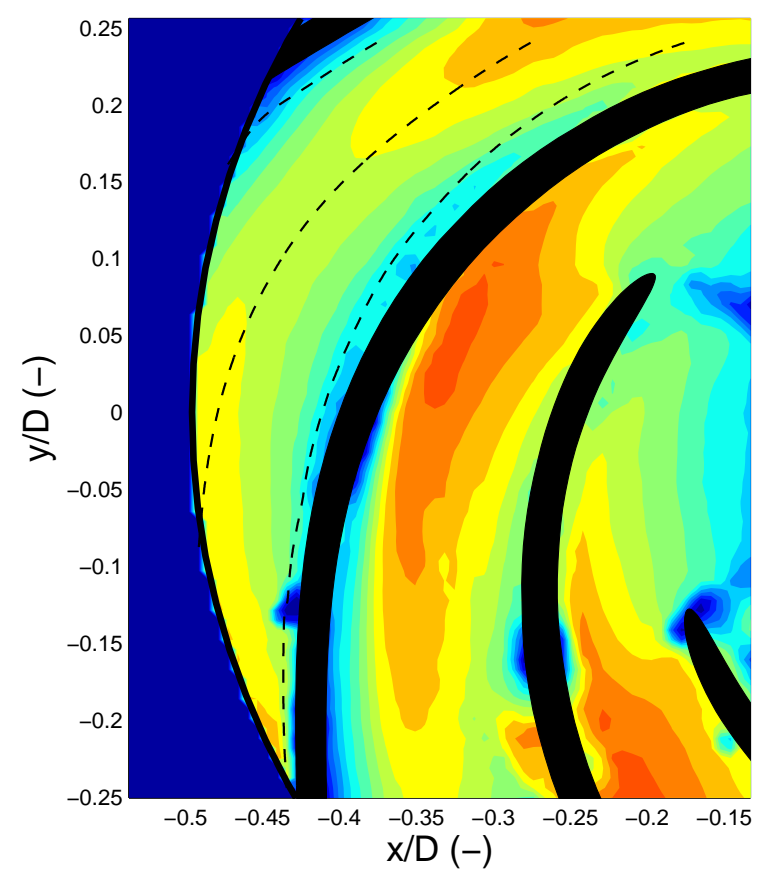

(c) measured flow field near shroud

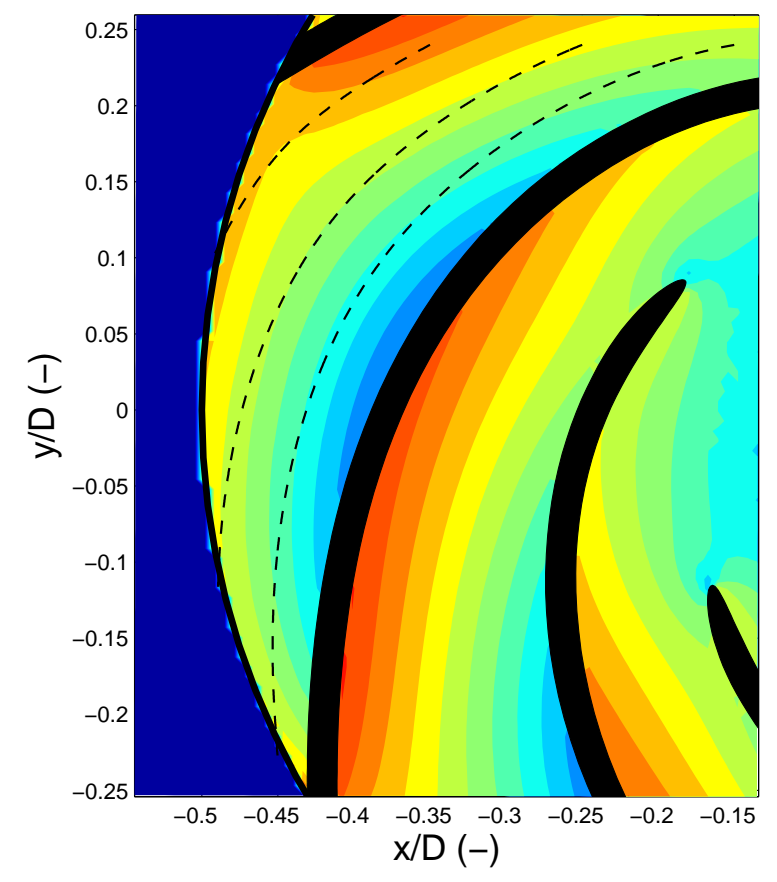

(b) computed flow field near hub

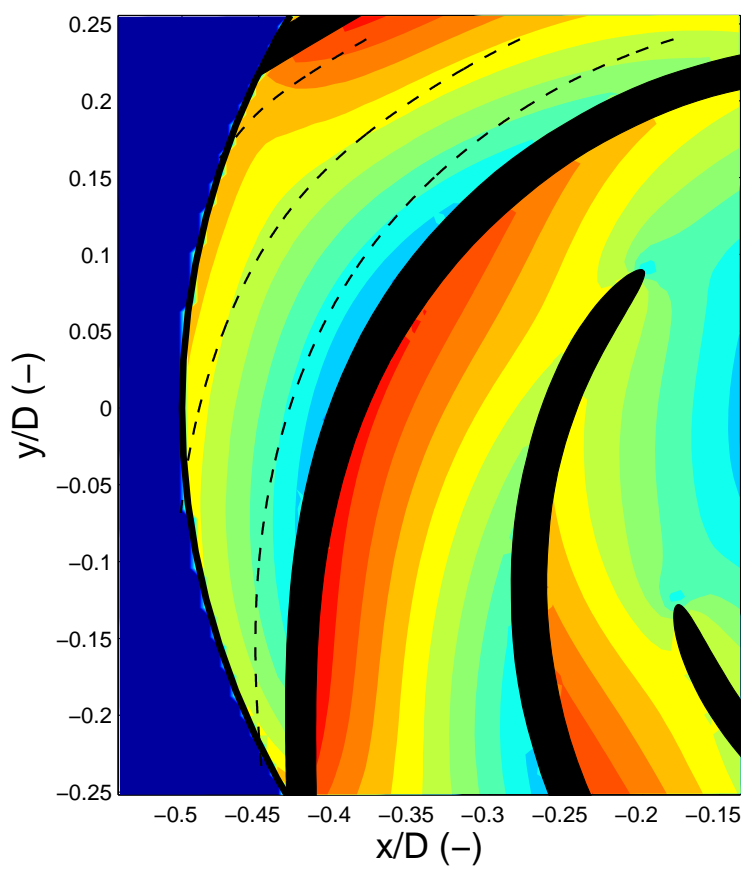

(d) computed flow field near shroud

Figure 5.31: Contour plots of measured and computed relative velocity, $w / u_{t e}$, near hub $(z / b=0.35)$ and shroud $(z / b=0.85)$ at $Q=1.2 Q_{d}$. Streamlines, with starting points at $y / D=0.24$, are given for the upper channel with a dashed line. 


\subsection{4 $Q=1.5 Q_{d}$}

Finally, measurements have been performed at a flow rate of $Q=1.5 Q_{d}$ and the measured and computed relative velocities are shown in both planes in Fig. 5.33. The distributions of relative velocity in the measurement plane near the hub and near the shroud are shown in Fig. 5.32 at a radius of $r / D=0.4$. Again the situation is very much comparable to that at the design point, $Q=Q_{d}$ and at $Q=1.2 Q_{d}$. In the plane near the hub larger velocities are measured than predicted, and no wake exists at the suction side. For the plane near the shroud, a wake region is present at the suction side. However, it does seem that the wake region is smaller when compared to the measurements at lower flow rates.

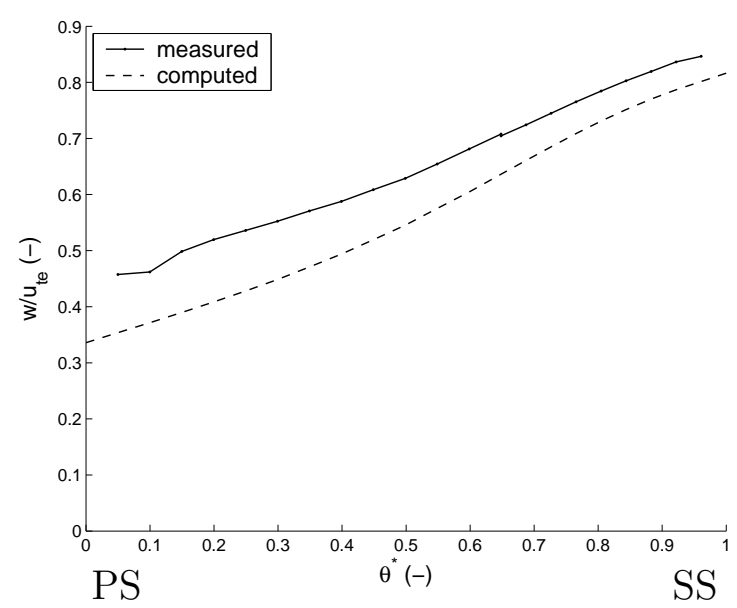

(a) near the hub, $z / b=0.35$

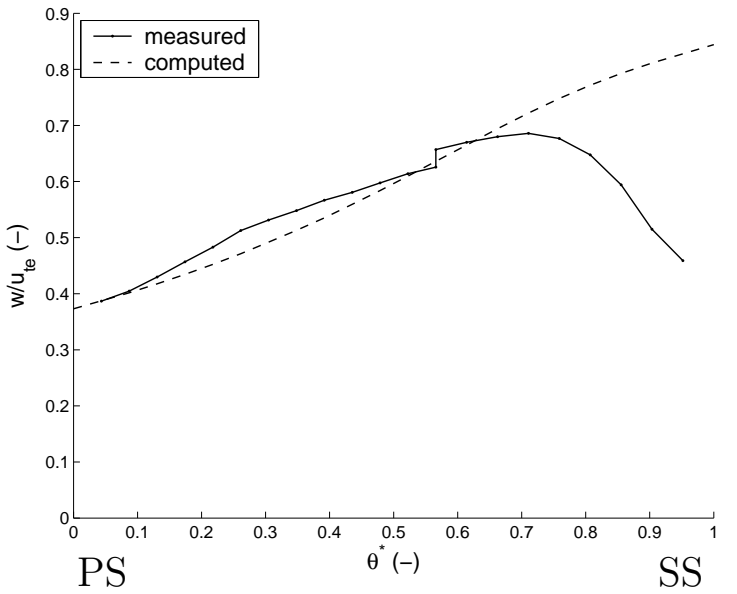

(b) near the shroud, $z / b=0.85$

Figure 5.32: Measured and computed profiles of relative velocity along a circular arc at $r / D=$ 0.4 for $Q=1.5 Q_{d}$. The profiles consist of data from two impeller channels, the transition is at $\theta^{*}=0.64$ for the hub and at $\theta^{*}=0.57$ for the shroud.

An interesting observation is that the predictions by the potential flow model at this flow rate are better than those at $Q=0.5 Q_{d}$. At $Q=0.5 Q_{d}$ the wakes at the suction side, both in the plane near the hub and in the plane near the shroud, lead to large differences between computations and measurements. At high flow rates the suction side wake effects are much smaller, i.e. they are only present at the shroud and are reduced in size, leading to smaller differences. 


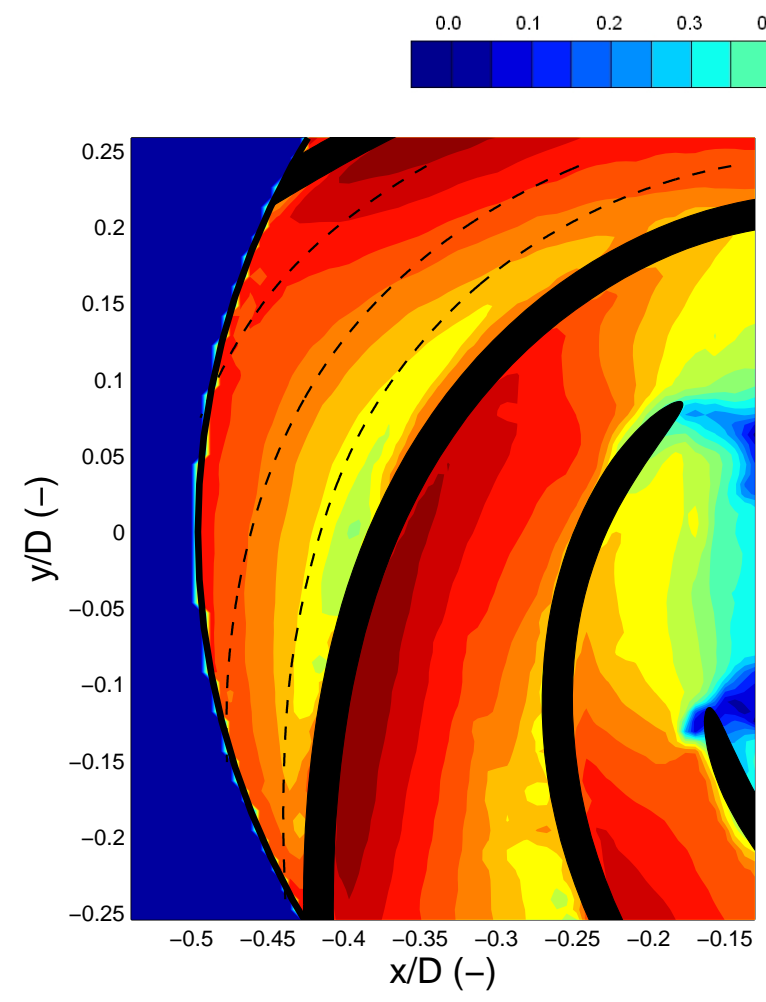

(a) measured flow field near hub

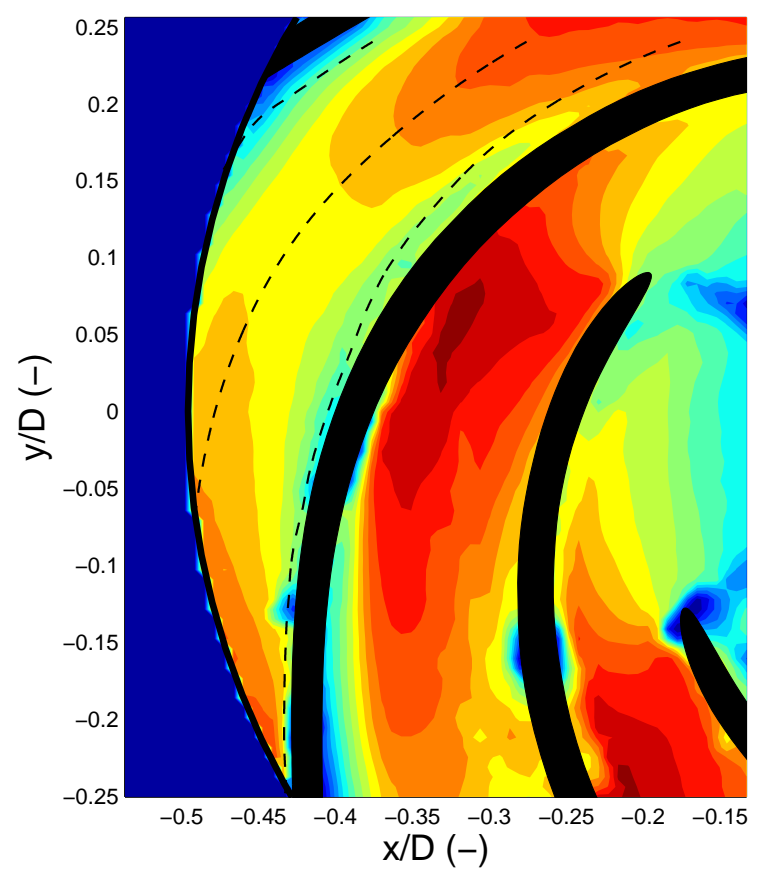

(c) measured flow field near shroud

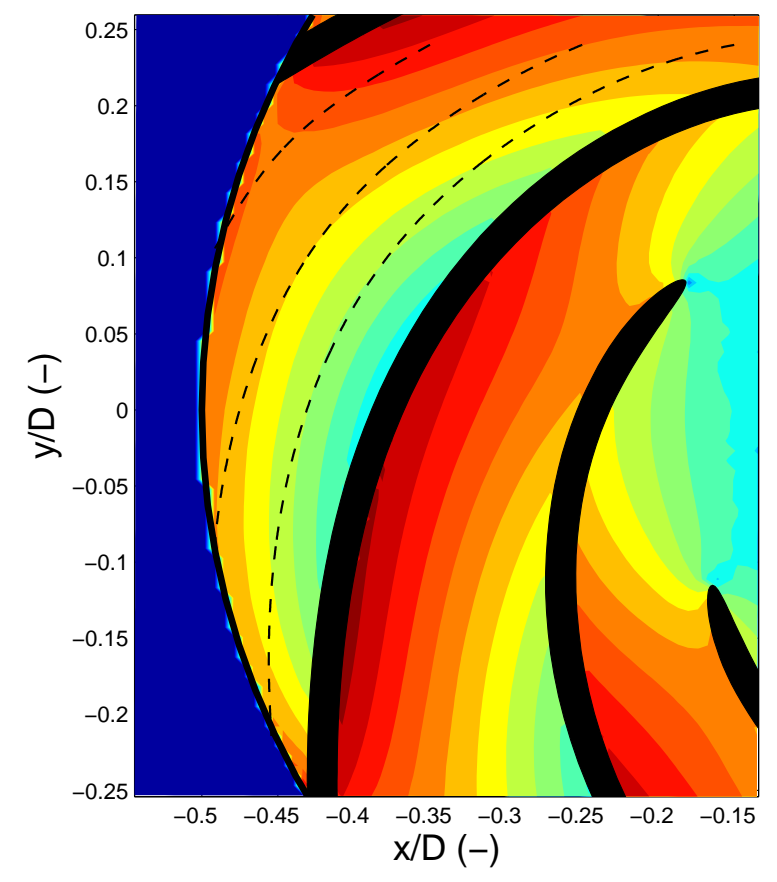

(b) computed flow field near hub

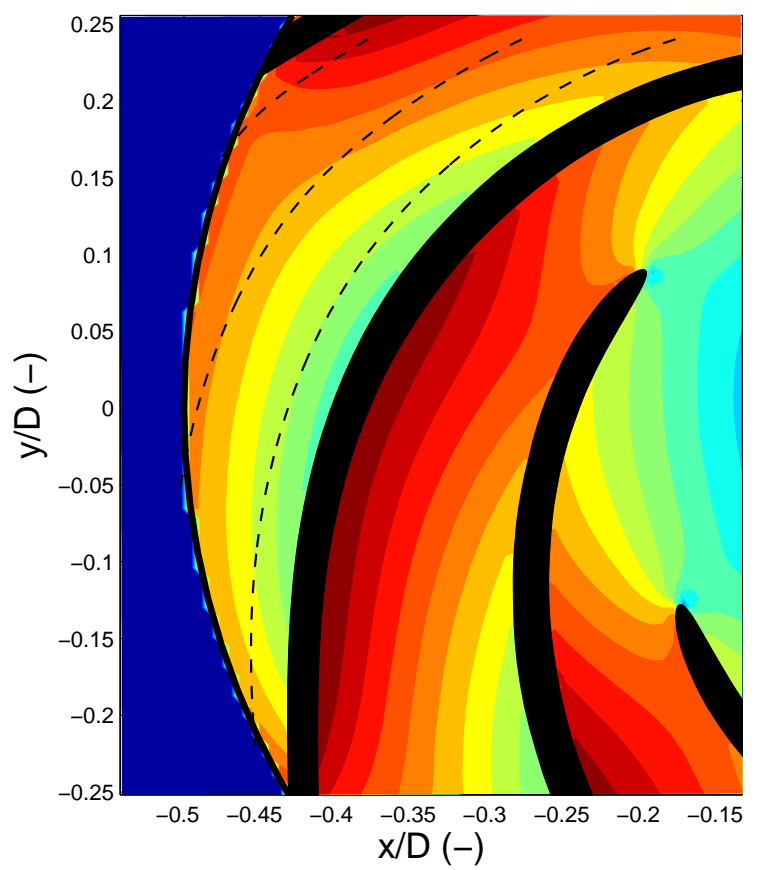

(d) computed flow field near shroud

Figure 5.33: Contour plots of measured and computed relative velocity, $w / u_{t e}$, near hub $(z / b=0.35)$ and shroud $(z / b=0.85)$ at $Q=1.5 Q_{d}$. Streamlines, with starting points at $y / D=0.24$, are given for the upper channel with a dashed line. 


\subsection{Discussion}

In sections 5.5 and 5.6 the measurements have been presented and compared to the computations for design and off-design conditions, respectively. The main observations for the results at design conditions are

- In the plane near the hub the measured velocity profiles are qualitatively similar to those predicted by the computations. Low relative velocities are observed at the pressure side, increasing to high relative velocities at the suction side.

- In the plane near the hub the relative velocities are higher than those predicted by the computations.

- In the plane near the shroud there is a qualitative difference between measurements and computations. A region of low velocity is observed at the suction side, which increases in size towards the trailing edge. This results in a so-called jet-wake flow pattern.

- The average relative velocity near the shroud is lower than predicted due to the drop in velocity at the blade suction side.

For off-design conditions it is observed that for higher flow rates, $Q>Q_{d}$, the same conclusions can be drawn for the velocity distributions as for design condition $Q=Q_{d}$. However, the wakes seem to become smaller for increasing flow rates. For lower flow rates, $Q<Q_{d}$, not only in the plane near the shroud, but also in the plane near the hub a region of low velocity is detected at the suction side. Especially at $Q=0.5 Q_{d}$ the differences between computations and experiments are large. The measured velocity profile in the plane near the shroud at design condition is sketched Fig. 5.34, where it is compared to the predicted inviscid-flow velocity profile.

The measured relative velocity in the plane near hub and that near the shroud are plotted in Fig. 5.35 along a circular arc at $r / D=0.4$, in order to investigate whether the suction side wake regions decrease in size with increasing flow rate, where the maximum velocity for each flow rate considered is indicated by a triangle. From these plots it is indeed concluded that at the shroud the suction side wake is always present and the size of the wake at this radius decreases with increasing flow rate. Near the hub the wake effect is only visible, at this radius, for the lowest considered flow rate.

The observed deviations from the inviscid flow predictions are considered to be caused by secondary flow effects. Therefore, secondary flow is discussed next, followed by a comparison with results of impeller flow measurements from literature.

\subsubsection{Secondary flow}

In this thesis the flow inside impellers is described by the augmented potential flow model (see chapter 2). This inviscid flow model does not account fully for the presence of boundary layers near walls. In reality the presence of such boundary layers in impellers is known to induce flows perpendicular to the main flow. Such flows are referred to as secondary flows. These secondary flows are the result of wall curvature and impeller 

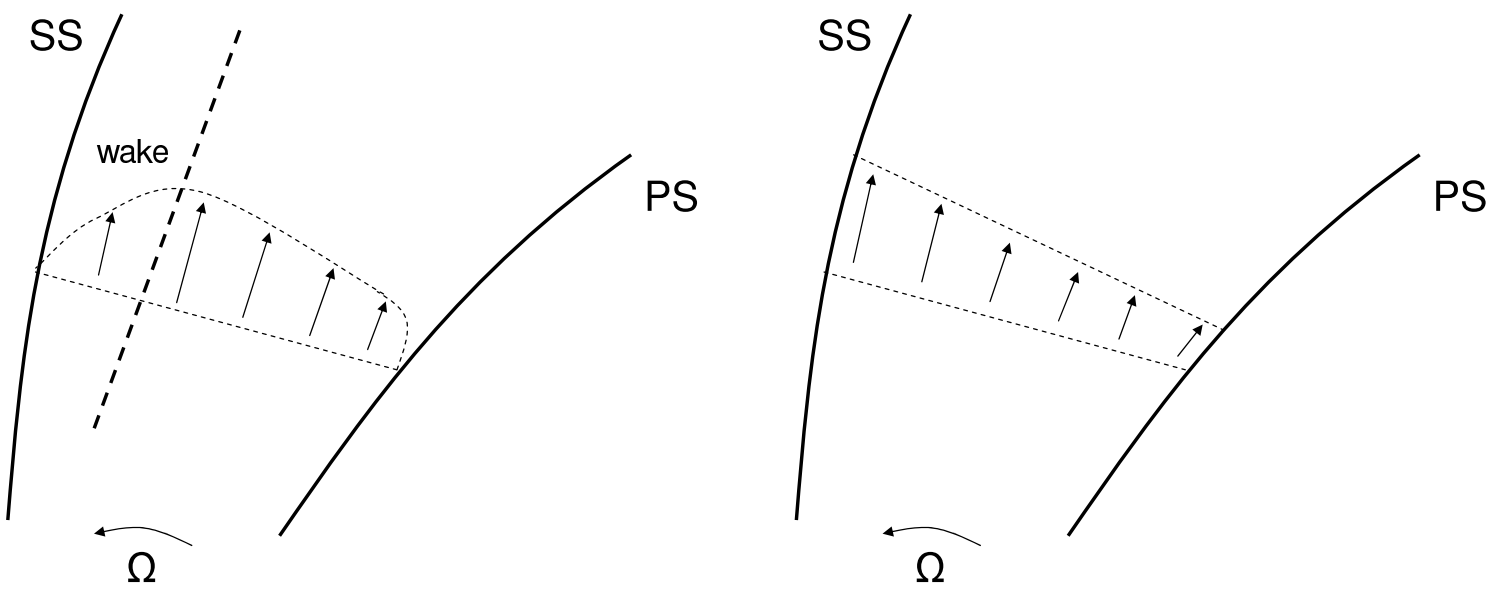

Figure 5.34: Sketch of the wake with low relative velocity near the suction side, as observed in the measurements in the plane near the shroud at design condition (left) compared with a sketch of an inviscid flow pattern (right). PS indicates the pressure and SS the suction side of the blade.

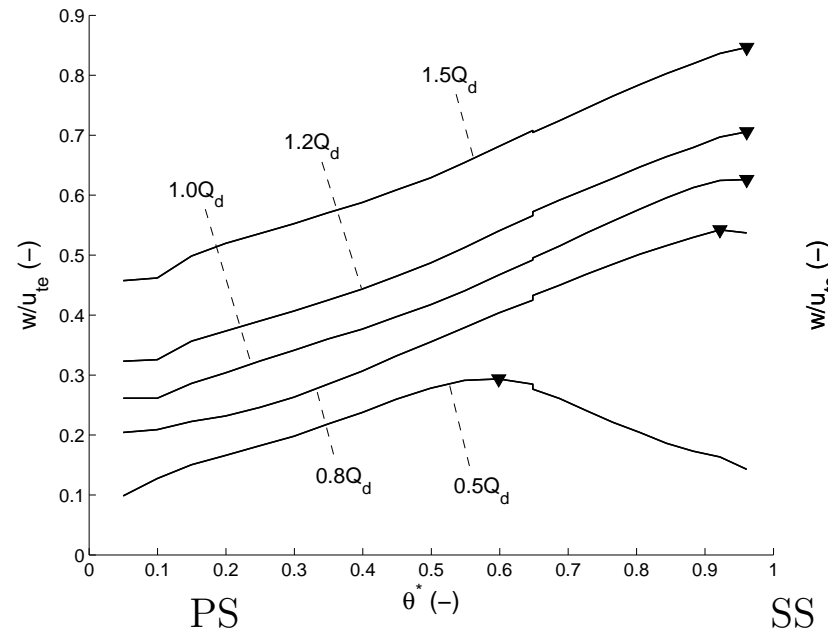

(a) near the hub, $z / b=0.35$

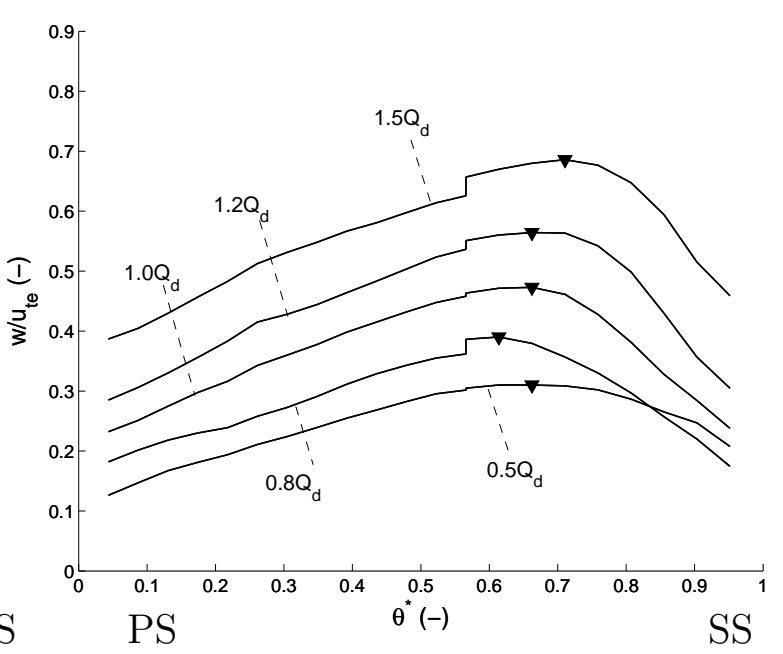

(b) near the shroud, $z / b=0.85$

Figure 5.35: Measured profiles of relative velocity in the plane near hub $(z / b=0.35)$ and near the shroud $(z / b=0.85)$ along a circular arc at $r / D=0.4$ for different flow rates. The triangles indicate the locations of maximum velocity. The measured profiles consist of data from two impeller channels, the transition is at $\theta^{*}=0.64$ for the hub and at $\theta^{*}=0.57$ for the shroud.

rotation. For centrifugal impellers two wall curvatures are important, i.e. the axial-toradial turn at the shroud and the blade curvature. Overviews of secondary flows are given in [11] and more specifically for turbomachines in [15]. The secondary flows that occur in the present measurements are discussed here.

Before discussing the secondary flow effects occurring in the impeller a definition of directions is given in Fig. 5.36. Here a quasi 2D boundary layer is sketched on a wall 


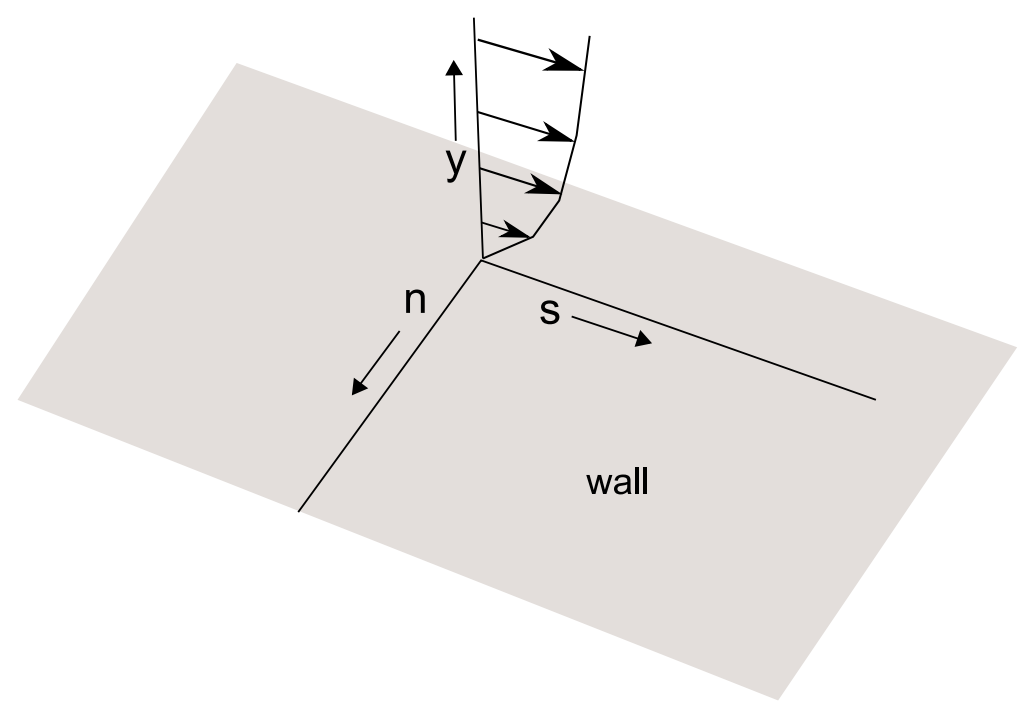

Figure 5.36: A boundary layer on a wall. $s$ is in the direction of the main flow, $y$ is normal to the wall and $n$ is normal to $s$ and $y$.

surface. The main flow is in $s$-direction. The $y$-direction is directed normal to the wall surface. Finally, the $n$-direction is normal to the $s$ - and $y$-direction. These directions are used in the subsequent paragraphs.

The first secondary flow effect that might play a role is caused by the axial-to-radial curvature at the shroud. For this purpose we will consider the influence of this curvature on the flow in the boundary layers at the blade pressure and suction side. A fluid particle in a local frame of reference moving along the axial-to-radial turn will experience a centrifugal force which is in balance with the pressure gradient [11]

$$
\frac{\partial p}{\partial n}=\frac{\rho w^{2}}{R_{C}}
$$

where $R_{C}$ is the radius of curvature of the stream line and $w$ the relative velocity. Inside the boundary layer the pressure gradient $\partial p / \partial n$ does not vary in the $y$-direction, i.e. normal to the wall [11]. Since the velocity $w$ is lower inside the pressure and suction side boundary layers, the radius of curvature $R_{C}$ must also be smaller to satisfy Eqn. (5.26), with $\partial p / \partial n=$ constant. The result is that inside the boundary layers at pressure and suction side a secondary flow is induced towards the shroud. In other words, low momentum fluid is carried from the pressure and suction side boundary layers towards the shroud. This secondary flow phenomenon caused by the axial-to-radial turn is sketched in Fig. 5.37 in situation $a$, and is considered to be responsible for the low velocities near the shroud, observed in the measurements.

The second secondary flow effect that is considered is that of blade curvature. In this situation the influence of the boundary layers at the hub and shroud are considered. In this case $n$ is in the direction from pressure to suction side. For similar considerations as described in the previous paragraph, a fluid element inside the hub and shroud boundary layers will move to a smaller radius of curvature, due to the low velocity inside the boundary layers. This means that fluid particles will tend to move towards the blade 


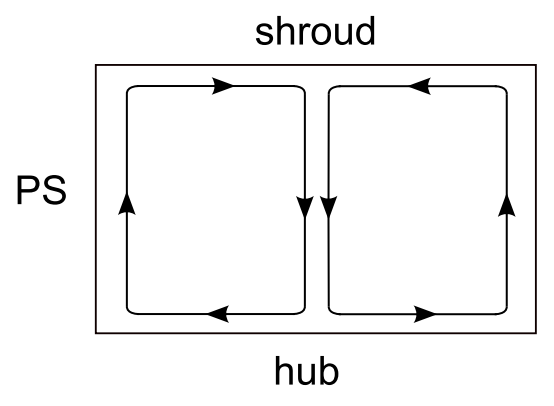

(a) axial-to-radial turn effect

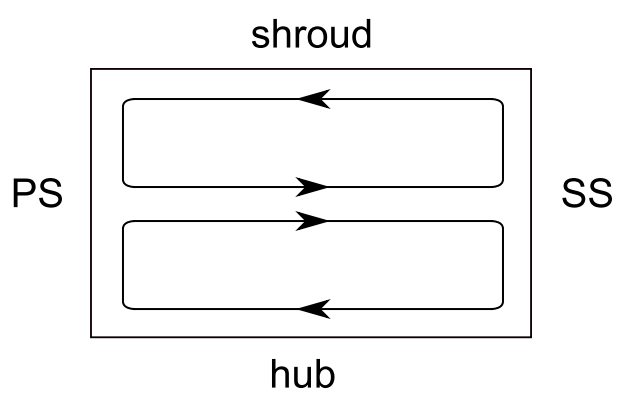

(b) blade curvature effect

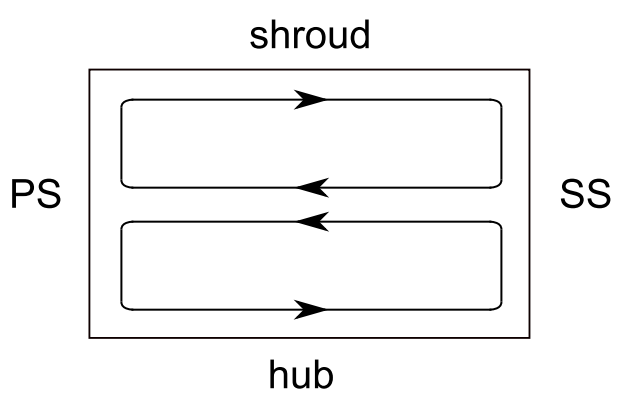

(c) impeller rotation effect

Figure 5.37: Secondary flow phenomena in centrifugal pump impellers, schematic frontal view of an impeller channel. Picture after [48]. PS indicates the pressure side and SS the suction side, respectively.

pressure side for backward curved blades. This situation is sketched partly in Fig. 5.37 situation $c$, together with another secondary flow effect which counteracts this blade curvature effect, which will be discussed now.

Apart from the blade curvature effect, another important secondary flow effect that occurs is caused by the impeller rotation. For this purpose an impeller with straight blades is considered, so that blade curvature plays no role. The secondary flow effect caused by the boundary layers on the hub and the shroud surface is considered, like for the blade curvature effect, and therefore $n$ is in the direction from pressure to suction side. A fluid particle in a rotating frame of reference inside the impeller will experience a Coriolis force which is in balance with the pressure gradient

$$
\frac{\partial p}{\partial n}=-2 \rho \Omega w
$$

where $\Omega$ is the rotational speed of the impeller. The Coriolis force is directed opposite to the impeller rotation and therefore is directed towards the pressure side. Inside the hub and shroud boundary layers the relative velocity is lower and hence the Coriolis force is smaller than in the core flow, since the pressure gradient $\partial p / \partial n$ is constant as was stated previously. Therefore, effectively inside the hub and shroud boundary layers a secondary flow is induced towards the suction side. This situation is sketched in Fig. 5.37 in situation $b$ and as one of the effects occurring in situation $c$.

Note that the impeller rotation thus induces a secondary flow effect contrary to the effect of blade curvature for backward curved blades. In order to analyze which effect 
is dominant in a particular centrifugal impeller the Rossby number $R o$ is defined, which gives the ratio between centrifugal and Coriolis force

$$
R_{o}=\frac{w}{2 \Omega R_{C}}
$$

Note that the factor 2 is sometimes omitted from the definition of the Rossby number. When $R_{O}<<1$ the Coriolis force is dominant and thus the rotational effect is more pronounced, leading to an average effective flow of low momentum fluid towards the suction side in the hub and shroud boundary layers. This phenomenon is considered to be the origin of the wake observed at the suction side, since in the present measurements the Rossby number varies roughly between 0.3 near the leading edge and 0.2 near the trailing edge. For most centrifugal pump impellers $R o<<1$ and consequently the jet-wake flow pattern with the wake at the suction side is a frequently observed flow phenomenon.

Summarizing, the axial-to-radial turn induces a secondary flow effect which results in the transport of low momentum fluid towards the shroud and hence is considered to be responsible the observed lower relative velocities at the shroud. Secondly, the rotation of the impeller induces a secondary flow effect, which is dominant over the effect of blade curvature when $R o<<1$. This secondary flow effect transports low momentum fluid towards the suction side for backward curved blades. The suction-side wake, which is observed on the plane near the shroud for all flow rates considered and at the hub for low flow rates, is attributed to this effect. For a more extensive discussion of these secondary flow effects the reader is reffered to [2].

However, other effects might also contribute to a decrease in velocity at the shroud. First of all, in the computations it is assumed that a uniform velocity profile is present in the central tube, upstream of the impeller. Preliminary PIV measurements have shown that this velocity profile is not as uniform as was expected. This is possibly caused by the diverging section in the Venturi. The measured profile in the central tube is shown in Fig. 5.38, where it is included in a sketch of the experimental setup. This profile should be considered as a qualitative indication, since it was not possible to get a completely sharp image of the seeding particles and since the velocity profile has not been corrected for diffraction of scattering light in the radial direction due to the curvature of the tube wall. This diffraction plays an important role, especially near the walls of the central tube. Taking these limitations into account, one can still see that the velocity in the center is higher than near the walls. This can lead to the situation sketched in Fig. 5.38 where the lower velocity at the shroud is partially caused by the low velocity near the wall of the central tube.

Another phenomenon that might play a roll is that when the fluid moves from the central tube into the impeller, it has to make a sharp turn along the axial-to-radial turn, and this could lead to a region of low velocity near the shroud. The measurements have shown that this is not a separated flow region, since no region with reversed-flow has been observed. 


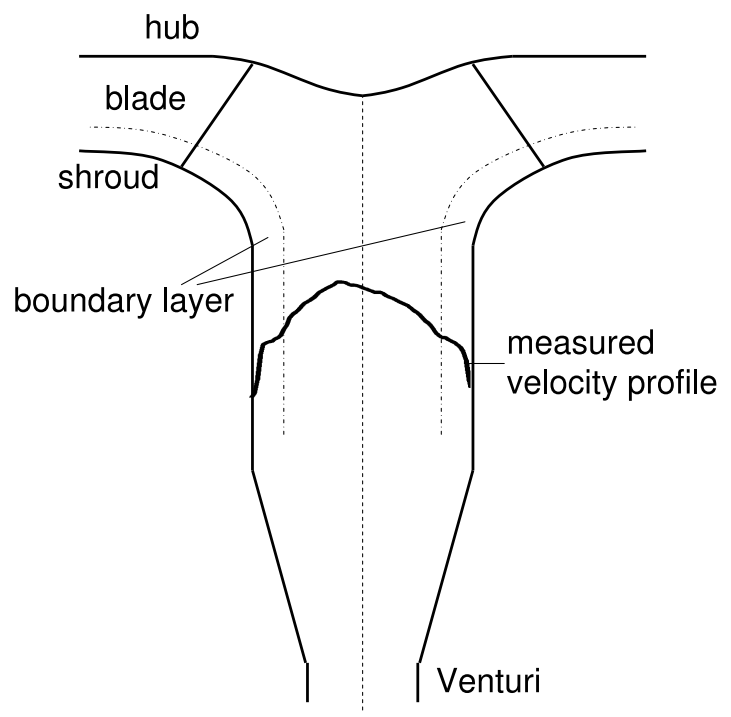

Figure 5.38: Sketch of the measured velocity profile in the central tube showing the influence on the velocity profile in the impeller.

\subsubsection{Comparison with literature}

In this section velocity measurements from literature are compared with the results of the present measurements. It is investigated if similar flow patterns due to secondary flow effects have been observed. Note that the focus is here on general velocity measurements in impellers, whereas the focus in section 5.3.1 is on PIV measurements specifically. Distributions of relative velocity from literature are used here for comparison; they are summarized in appendix C.

Van Os [47] performed Laser Doppler Velocimetry (LDV) measurements in a mixedflow impeller. He measured the velocity profiles in 6 planes, more or less perpendicular to the main stream, from leading to trailing edge (see Fig. C.1). For the two planes closest to the trailing edge he reports a strong decrease of the velocity at the shroud suction side for the design flow rate. Furthermore, from measurements performed at off-design conditions he concludes that the location at which this wake area starts to form moves downstream for increasing flow rates, but that it never disappears completely. This is in accordance with the conclusion from the present measurements: the wake area decreases in size for increasing flow rate.

The LDV measurements by Eckardt [28] in a compressor impeller without back sweep (straight blades) also support the formation of a wake-like region without reversed flow at the suction side near the shroud, as is shown in Fig. C.2. Krain [44] performed measurements in a compressor impeller with backward curved blades and also reports lower velocities at the shroud surface. However, the wake formation at the suction side of that shroud side is not observed, but rather a region of low velocity in the center of the channel, see Fig. C.3. 
Furthermore, Wuibaut et al. [77, 78] also report the existence of a wake region near the shroud suction side for the radial SHF impeller, both for PIV experiments in air and for LDV measurements in water (see Fig. C.5). The LDV measurements presented by Visser [69] carried out in a fifteen bladed radial impeller are shown in Fig. C.6. These measurements are carried out at mid-height between hub and shroud. The formation of a suction-side wake is clearly observed.

Concluding, the general deviations observed from inviscid flow predictions in our measurements, are also observed in literature and are therefore of a general character. Most authors report the suction side wake formation, which is most pronounced at the shroud. As discussed, these effects are considered to be the result of secondary flow effects. These effects, which have a viscous origin, are not included in the inviscid flow model used in this thesis. 


\subsection{Conclusions and recommendations}

A newly designed setup for testing centrifugal pump impellers has been designed and realized. The setup is largely transparent so that detailed flow phenomena can be measured by use of PIV.

The setup in its current state has an operating range of up to $200 \mathrm{rpm}$ and flow rates up to twice the design flow rate can be realized at the rotational speed considered. The flow rate is measured with a calibrated Venturi flow meter. At the design flow rate there is a $3-4 \%$ discrepancy between the flow rate predicted by the measured pressure difference of taps 1 and 2 and that predicted using the values measured at taps 2 and 3 . The pressure taps 1 and 2 are used for flow rate prediction in the measurements, since the influence of the impeller is expected to be smallest there. The static pump head has been measured and compared to the inviscid-flow computation. There is a small difference between the measured and computed inviscid-flow static head. This can be attributed to effects of viscosity, which are not considered in the inviscid flow model.

PIV measurements have been carried out successfully in the optimized impeller. The velocity fields do not exhibit peak locking and the time-averaged velocity profiles show a good match between different impeller channels. Hence, the free impeller assumption, in which only a single impeller channel is considered for the flow computation, is validated. Typical velocity patterns occurring in turbomachines, with a low relative velocity at the pressure side and a high relative velocity at the suction side have indeed been measured. The relative velocity at the hub is somewhat higher than predicted by the computations. The relative velocity at the shroud is somewhat lower than predicted, especially near the suction side where a region of low relative velocity is present. This region is labeled a wake region. The lower velocity at the shroud is mainly attributed to secondary flow effects caused by the axial-to-radial turn of the flow channel. The suction-side wake is accounted for by the secondary flow caused by impeller rotation. The general observed flow patterns are supported by various sources from literature.

For future measurements it is advised to create a more uniform velocity profile in the central tube upstream of the impeller. This can be facilitated by inserting an extra flow straightener in the Venturi following the diverging section or by redesigning parts of the central tube. It is also recommended to reduce the circulation in the main vessel by inserting vertical plates.

Furthermore, it is advisable to perform more PIV measurements in planes between hub and shroud. In this thesis only 2 planes are considered, one near the hub and one near the shroud. This was largely due to time restrictions. By measuring at more heights between hub an shroud a more detailed view of the impeller flow is obtained.

Moreover, in order to measure the velocity profile in the central tube more accurately, it is advised to use a rectangular water prism, so that diffraction of light is less of an issue. In this way a quantitative estimation of the flow rate can also be achieved and used to investigate which combination of pressure taps gives the best flow rate prediction.

It is strongly recommended to carry out measurements at higher Reynolds numbers by increasing the rotational speed. Currently, this could not be done due to the entrapment of bubbles in the setup. From the comparison between measurements carried out at 
$75 \mathrm{rpm}$ and those performed at $150 \mathrm{rpm}$ it did appear that the wake area decreases somewhat in size with increasing Reynolds numbers. However, the difference is only small. More measurements are needed to investigate whether the suction side wake area indeed decreases in size with increasing Reynolds number. This is relevant since industrial impellers generally operate at higher Reynolds numbers than those considered in the present measurements.

Concluding, the potential flow model is capable of predicting flow behavior inside the impeller reasonably well. Global parameters like static pump head can be estimated quite well. The general flow phenomenon of low relative velocity at the pressure side and high relative velocity at the suction side, caused by the counter-vortex, are indeed measured. However, detailed flow phenomena caused by secondary flow effects, like the encountered wake at the shroud suction side can not be predicted with this model. To capture such phenomena a secondary flow model has to be added to the potential flow model, or a more detailed viscous flow model, with appropriate turbulence model, is needed. The boundary layer losses predicted by the augmented potential flow model, using a semiempirical relation, is likely to be too low, since in reality the boundary layers are thicker than expected. 


\section{CHAPTER 6}

\section{Discussion}

In this chapter the conclusions from the previous chapters are summarized and recommendations for further research are formulated. The main goal of this work has been to develop CFD-based design methods for turbomachine impellers that are based on results of flow computations. For this purpose two methods have been developed: an inversedesign method, see chapter 3 and an optimization method for centrifugal impellers, see chapter 4. Both design methods make use of the augmented potential flow model to describe the hydrodynamics inside an impeller. This flow model has been introduced in chapter 2 of this thesis. The potential flow model is an inviscid flow model and does not consider viscous effects directly. Therefore, measurements have been performed in an optimized impeller to validate the velocity profiles computed by the potential-flow method. Particle Image Velocimetry (PIV) measurements have been carried out for this purpose, see chapter 5 .

A major advantage of the inverse-design method is that the three-dimensional blade curvature distribution is obtained, that gives the prescribed performance. By prescribing an appropriate loading function, a three-dimensional impeller geometry is obtained with zero-incidence at the leading edge and with the pump head prescribed by the designer. It has been shown in chapter 3 that by shifting the prescribed loading towards the leading or trailing edge, performance parameters like boundary-layer losses, velocity loading and cavitation inception can be improved. The improvement of these performance parameters, by modifying the loading distribution, can also be carried out automatically by making use of an optimization method, leading to a so-called inverse-optimization which is put forward in chapter 4 in section 4.4 .

An interesting situation that might occur is that a designer tries to increase the pump head of a certain impeller using an inverse-design method, simply by changing the prescribed pump head. However, one needs to consider that by changing the pump head drastically, ideally a different meridional shape has to be selected as well, due to the change in specific speed. In the inverse-design method only the blade curvature distribution is modified however, and the meridional geometry is fixed. Therefore it is important 
that a designer always takes a critical look at the obtained inversely-designed impeller blades.

A possible future addition to the inverse-design method is the use of a prescribed thickness distribution instead of considering blades with zero thickness as done here. A first step in this direction has been made by Bijleveld [5], who implemented a prescribed blade thickness distribution for two-dimensional geometries. The current method uses a structured mesh generator. It is likely that an unstructured 3D mesh generator is needed for this purpose. Furthermore, in chapter 3 it has been shown that the inverse-design method is first order accurate due to the low accuracy of the velocity determination near domain boundaries, using the superconvergent patch recovery method. Therefore, it is recommended to improve this recovery technique or use a different method in order to increase the order of accuracy of the inverse-design method.

The direct optimization method for centrifugal pump impellers has been shown to be an extremely useful tool in the design of impellers. The utilized optimization algorithm is the Differential Evolution (DE) method. By including relevant geometrical aspects in the parameterization and formulating a cost function based on performance parameters of interest, impellers are obtained with increased performance for a specified operational range. In chapter 4 an existing radial pump impeller has been redesigned using the developed optimization method. The included parameters describe the blade and shroud curvature, as well as the number of blades of the impeller. Other geometric parameters can be added easily to such optimizations. For instance hub curvature, leading edge location, blade thickness distribution, or blade stacking at the trailing edge might be considered. The objectives included in the cost function were the pump head, boundary-layer losses and cavitation parameters. Other objectives can be added as well, such as the maximum velocity loading on the blades or the size of the through flow area, which is an important design objective for dredge pumps. The selection of weight coefficients for the (conflicting) objectives and bounded ranges for the parameters is a delicate procedure, which determines the possible outcome of the optimization process. Therefore, the designer has to choose these wisely.

The optimization method can be improved further by including a response surface method or an artificial neural network for the description of the cost function, see for example $[23,34,57]$. By using such a method, an approximation of the cost function is obtained, which can be optimized at low computational cost. Therefore, by utilizing such methods, the required flow computations can be reduced significantly. Furthermore, in the direct optimization impeller geometries with back-flow are penalized with a back-flow factor, which is currently of the on/off type. It might be considered for future optimizations to quantify this back-flow effect in more detail, for example by considering the size of the back-flow region in the impeller. Moreover, in the current formulation of the cost function the objectives are summed into a lumped cost function. It might be considered for future optimizations to perform true multi-objective optimizations considering Pareto fronts [1].

It is interesting to make a comparison between both developed design methods and give an overview of advantages and disadvantages of each of the methods. These are summarized below 
- The inverse-design method requires much less computational effort when compared to the direct optimization method.

- The pump head needs to be included in the direct optimization method in the cost function, while this is not the case for the inverse-design method.

- Off-design performance is taken into account in the direct optimization method. This is not the case for the inverse-design method.

- The optimization method is more flexible, i.e. additional parameters and objectives can be added easily, whereas the inverse-design method only considers blade curvature.

Each of the developed methods has its advantages and might be used at a certain point in the design process. For example, the optimization method might be used for a complete design of the impeller, with the focus on the meridional shape and the number of blades. The optimized meridional shape and number of blades might then be used as input for the inverse-design method, in order to find the blade curvature distribution. For a fast inverse analysis the designer may also consider the quasi three-dimensional approach, which only requires a single flow computation (see section 3.3.1).

The PIV measurements presented in chapter 5 show that the potential flow model gives a reasonable prediction of the flow field inside centrifugal impellers and predicts the static pump head quite well, apart from a small difference due to viscous flow effects. However, secondary flow effects, resulting from viscous effects inside boundary-layers near walls, also play an important role in the hydrodynamics in impellers. These secondary flow effects lead to the formation of a wake at the suction side, which is most pronounced near the shroud. This wake region results in a blockage effect of the flow, which in turn leads to an increase in velocities outside the wake region. Similar observations have been made by other authors in literature and it is concluded that these secondary flow effects are frequently present in turbomachines and that for a good detailed description of the flow inside the impellers these effects need to be accounted for. In the current inviscid flow model they are not considered. Therefore, it is advised that for future work either the potential flow model is extended with a secondary flow model or that a transition is made to a viscous flow model, for example a Reynolds-Averaged Navier Stokes (RANS) method. Using a RANS method instead of an inviscid flow analysis will lead to a drastic increase in computational effort, and should only be carried out when needed.

In this work boundary-layer losses are estimated based on the computed inviscid flow fields by a semi-empirical relation, that uses the potential-flow field as input. The use of this approach is justified because the boundary-layers are relatively thin due to the high Reynolds numbers. However, the measurements have shown that the suction side wake comprises a relative large part of an impeller channel. This will most likely lead to larger boundary-layer losses than predicted by the semi-empirical relation which is used here.

The tested impeller has been optimized based on global parameters, i.e. pump head, boundary-layer losses and cavitation characteristics. In the current setup only the (static) pump head has been measured and a reasonable agreement was found between prediction and measurements. Since the optimized impeller is mainly improved with respect to 
cavitation parameters, it is advised to measure these characteristics as well and, if possible, also the efficiency of the impeller. The deviation from inviscid flow predictions is most pronounced at larger radii. Cavitation occurs generally at lower radii, since the pressure increases from leading to trailing edge. Therefore it is expected that the deviations from the flow model will not have a large influence on the predicted cavitation coefficients. A marked difference is expected in predicted and occurring boundary-layer losses however, since the boundary-layers due to secondary flow effects are relatively thick and most likely will lead to an increase in boundary-layer losses.

The employed potential-flow method is just one building block of the optimization method, used to describe the hydrodynamics inside the impeller and to calculate the cost function. This building block can easily be replaced by a different flow prediction method, which gives a more accurate description of the flow. For the optimization method there are thus ways to combine the advantages of a fast inviscid flow computation and a slower but more realistic viscous flow analysis by carrying out a limited number of viscous flow computations alongside the inviscid flow computations. Such an approach is labeled as a multi-fidelity method, see for example [4, 34]. As was referred to in the optimization chapter, the use of a response surface method or neural network can also be used to reduce the number of flow computations needed even further.

In the opinion of the author the use of computer-aided design methods will become more and more important in the coming decades. In the earlier stages of a design process, it is probably sufficient to use an inviscid flow model for this purpose. In this stage the developed inverse-design method and optimization method in their current state can play a role. For the final design stage, a flow model is needed which incorporates effects due to viscosity.

Finally it has to be noted that in this thesis the focus is on impeller design, whereas in reality the design of both impeller and diffuser are of importance. For some applications it might be desirable to carry out a combined design of impeller and diffuser, rather than splitting the design process in two separate parts. This is especially relevant at off-design conditions where complex flow features of the diffuser may influence the hydrodynamics in the impeller or vice versa. 


\section{Bibliography}

[1] A. Abraham, L. C. Jain, and R. Goldberg. Evolutionary multiobjective optimization. Springer, New York, 2005.

[2] K. van Andel. Particle image velocimetry of flow fields in a centrifugal pump impeller. MSc Thesis, University of Twente, April 2008.

[3] N. Antonakis. Optimization methods for centrifugal pump impellers. MSc Thesis, Delft University of Technology, July 2006.

[4] T.R. Barrett, N.W. Bressloff, and A.J. Keane. Airfoil shape design and optimization using multifidelity analysis and embedded inverse design. AIAA Journal, 44(9):20512060, 2006.

[5] H.A. Bijleveld. Extensions to an inverse design method for pump impellers. $M S c$ Thesis, University of Twente, November 2007.

[6] J.E. Borges. A three-dimensional inverse method for turbomachinery: Part 1-theory. Journal of Turbomachinery, 112(3):346-354, 1990.

[7] C.E. Brennen. Cavitation in pumps. CISM course: Fluid Dynamics of Cavitation and Cavitating Turbopumps, July 2005.

[8] S. Burguburu and A. le Pape. Improved aerodynamic design of turbomachinery bladings by numerical optimization. Aerospace Science and Technology, 7(4):277$287,2003$.

[9] S. Burguburu, C. Toussaint, C. Bonhomme, and G. Leroy. Numerical optimization of turbomachinery bladings. Journal of Turbomachinery, 126(1):91-100, 2004.

[10] S.L. Cao and Yu Z.Y. Peng, G.Y. Hydrodynamic design of rotodynamic pump impeller for multiphase pumping by combined approach of inverse design and cfd analysis. Journal of Fluids Engineering, 127(2):330-338, 2005. 
[11] T. Cebeci and J. Cousteix. Modelling and computation of boundary-layer flows. Springer-Verlag, New York, 1999.

[12] Y.D. Choi, K. Nishino, J. Kurokawa, and J. Matsui. PIV measurement of internal flow characteristics of very low specific speed semi-open impeller. Experiments in Fluids, 37(5):617-630, 2004.

[13] K.T. Christensen. The influence of peak-locking errors on turbulence statistics computed from PIV ensembles. Experiments in Fluids, 36(3):484497, 2004.

[14] D.G. Crighton. The Kutta condition in unsteady flow. Annual Review of Fluid Mechanics, 17:411-445, 1985.

[15] N.A. Cumpsty. Compressor Aerodynamics. Longman Scientific and Technical, Cambridge, 1989.

[16] S. Damle, T. Dang, J. Stringham, and E. Razinsky. Practical use of three-dimensional inverse method for compressor blade design. Journal of Turbomachinery, 121(2):321$325,1999$.

[17] K. Daneshkhah and W. Ghaly. Aerodynamic inverse design for viscous flow in turbomachinery blading. Journal of Propulsion and Power, 23(4):814-820, 2007.

[18] T. Dang, S. Damle, and X. Qiu. Euler-based inverse method for turbomachine blades, part 2: Three-dimensional flows. AIAA Journal, 38:2007-2013, 2000.

[19] T. Dang and V. Isgro. Euler-based inverse method for turbomachine blades, part 1: Two-dimensional cascade. AIAA Journal, 33:2309-2315, 1995.

[20] J.P. DeCarlo. Fundamentals of Flow Measurement. Instrument Society of America, Foxboro, Massachusetts, 1984.

[21] N.G. Deen. Introduction to particle image velocimetry (PIV). Educational notes, University of Twente, 2002.

[22] A. Demeulenaere and R. van den Braembussche. Three-dimensional inverse method for turbomachinery blading design. Journal of Turbomachinery, 120(2):247-255, 1998.

[23] A. Demeulenaere, A. Purwanto, A. Ligout, C. Hirsch, R.J.H. Dijkers, and F. Visser. Design and optimization of an industrial pump: Applications of genetic algorithms and neural network. ASME 5th International Symposium on Turbomachinery, FEDSM2005-77487:1-9, 2005.

[24] J.D. Denton. Loss mechanisms in turbomachines. Journal of Turbomachinery, 115:621-656, 1993.

[25] R.J.H. Dijkers and J.H.G. op de Woerd. Personal communication. Flowserve Corporation, Hengelo Nederland, 2004. 
[26] S.L. Dixon. Fluid Mechanics and Thermodynamics of Turbomachinery. ButterworthHeinemann, Oxford, 1997.

[27] M. Drela and H. Youngren. Xfoil. http://web.mit.edu/drela/Public/web/xfoil, 2001.

[28] D. Eckardt. Detailed flow investigations with a high-speed centrifugal-compressor impeller. Journal of Fluids Engineering, 98(3):390-402, 1976.

[29] Engineers Edge. Pump impeller types. www.engineersedge.com/pumps.

[30] R. Eppler. PROFILE - the eppler airfoil code. http://www.pdas.com/eppler.htm, 2000.

[31] B.P.M. van Esch. Simulation of three-dimensional unsteady flow in hydraulic pumps. PhD Thesis, University of Twente, 1997.

[32] B.P.M. van Esch and N.P. Kruyt. Hydraulic performance of a mixed-flow pump: Unsteady inviscid computations and loss models. Journal of Fluids Engineering, 123(2):256-264, 2001.

[33] V. Feoktistov. Differential Evolution - in search of solutions. Springer, New York, 2006.

[34] A.I.J. Forrester, A. Sobester, and A.J. Keane. Multi-fidelity optimization via surrogate modelling. Proceedings Of The Royal Society A - Mathematical Physical And Engineering Sciences, 463:3251-3269, 2007.

[35] Carlson R.E. Fritsch, F.N. Monotone piecewise cubic interpolation. SIAM Journal on Numerical Analysis, 17(2):238-246, 1980.

[36] F. Frohlig, S. Caglar, and M. Gabi. Measurments in an unshrouded pump using stereo-particle-image-velocimetry. International Symposium on Rotating Machinery 12, ISROMAC12-2008-20030:1-12, 2008.

[37] K.G. Giannakoglou and J. Periaux, editors. Optimization methods and tools for multicriteria/multidisciplinary design. Von Karman Institute, November 2004.

[38] G.J. Goldstein. Fluid Mechanics Measurements. Taylor\&Francis, Minnesota, 1996.

[39] A. Goto and M. Zangeneh. Hydrodynamic design of pump diffuser using inverse design method and CFD. Journal of Fluids Engineering, 124(2):319-328, 2002.

[40] W.R. Hawthorne, C. Wang, C.S. Tan, and J.E. McCune. Theory of blade design for large deflections: Part 1 - two-dimensional cascade. Journal of Turbomachinery, 106(2):346-353, 1984.

[41] C.M. Jang, P. Li, and K.Y. Kim. Optimization of blade sweep in a transonic axial compressor rotor. JSME International Journal Series B - Fluids and Thermal Engineering, 48(4):793-801, 2005. 
[42] H. Japikse, W.D. Marscher, and R.B. Furst. Centrifugal Pump Design and Performance. Concepts ETI Inc., Wilder, 1997.

[43] J. Jiang and T. Dang. Design method for turbomachine blades with finite thickness by the circulation method. Journal of Turbomachinery, 119(3):539-543, 1997.

[44] H. Krain. Swirling impeller flow. 32nd ASME Gas Turbine Conference and Exhibit, Paper 87-GT-19, 1987.

[45] N. Krause, K. Zahringer, and E. Pap. Time-resolved particle imaging velocimetry for the investigation of rotating stall in a radial pump. Experiments in Fluids, 39(2):192201, 2005.

[46] N.P. Kruyt, B.P.M. van Esch, and J.B. Jonker. A superelement-based method for computing unsteady three-dimensional potential flows in hydraulic turbomachines. Communications in Numerical Methods in Engineering, 15(6):381-397, 1999.

[47] M.J. van Os. On the flow and cavitation inception of mixed-flow impellers. PhD Thesis, University of Twente, 1998.

[48] N. Pedersen. Experimental investigation of flow structures in a centrifugal pump impeller using particle image velocimetry. PhD Thesis, Technical University of Denmark, 2000.

[49] N. Pedersen, P.S. Larsen, and C.B. Jacobsen. Flow in a centrifugal pump impeller at design and off-design conditions - part I: particle image velocimetry (PIV) and laser doppler velocimetry (LDV) measurements. Journal of Fluids Engineering, 125:61-72, 2003.

[50] G.Y. Peng. A practical combined computation method of mean through-flow for 3D inverse design of hydraulic turbornachinery blades. Journal of Fluids Engineering, 127(6):1183-1190, 2005.

[51] G.Y. Peng, S.L. Cao, M. Ishizuka, and S. Hayama. Design optimization of axial flow hydraulic turbine runner: Part 2 - multi-objective constrained optimization method. Interantional Journal for Numerical Methods in Fluids, 39(6):533-548, 2002.

[52] G.Y. Peng, S.L. Cao, M. Ishizuka, and S. Hayama. Design optimization of axial flow hydraulic turbine tunner: Part 1 an improved Q3D inverse method. Interantional Journal for Numerical Methods in Fluids, 39(6):517-531, 2002.

[53] S. Pierret, R.F. Coelho, and H. Kato. Multidisciplinary and multiple operating points shape optimization of three-dimensional compressor blades. Structural and Multidisciplinary Optimization, 33(1):61-70, 2006.

[54] B.T. Polyak. Introduction to optimization. Optimization Software Inc., New York, 1987. 
[55] K.V. Price, R.M. Storn, and J.A. Lampinen. Differential Evolution - a practical approach to global optimization. Springer, New York, 2005.

[56] M. Raffel, C.E. Willert, and J. Kompenhans. Particle Image Velocimetry: a Practical Guide. Springer Verlag, Berlin, 1998.

[57] M.M. Rai. Multi-objective optimization with differential evolution and neural networks. VKI lecture series on optimization methods and tools for multidisciplinary design, pages 1-22, November 2004.

[58] H. Schlichting. Boundary-Layer Theory. McGraw-Hill, New York, 1987.

[59] J.J. Schneider and S. Kirkpatrick. Stochastic Optimization. Springer, New York, 2006.

[60] M. Sinha, A. Pinarbasi, and J. Katz. The flow structure during onset and developed states of rotating stall within a vaned diffuser of a centrifugal pump. Journal of Fluids Engineering, 123(3):490-499, 2001.

[61] H. Spring. Affordable quasi three-dimensional inverse design method for pump impellers. Proceedings of the Ninth International Pump Users Symposium, pages 97110, 1992.

[62] H. Spring. Judge the pump hydraulic design through numbers before you buy. Proceedings of the Twelfth International Pump Users Symposium, pages 113-119, 1995.

[63] A.J. Stepanoff. Centrifugal and axial flow pumps. John-Wiley and Sons, 1964.

[64] R.M. Storn and K.V. Price. Differential evolution - a simple and efficient heuristic for global optimization over continuous spaces. Journal of Global Optimization, 11:341359, 1997.

[65] S.S. Talya, J.N. Rajadas, and A. Chattopadhyay. Multidisciplinary design optimization of film-cooled gas turbine blades. Mathematical Problems in Engineering, 5(2):97-119, 1999.

[66] C.S. Tan, W.R. Hawthorne, J.E. McCune, and C. Wang. Theory of blade design for large deflections: Part 2 - annular cascades. Journal of Engineering for Gas Turbines and Power, 106(2):354-365, 1984.

[67] W.T. Tiow, K.F.C. Yiu, and M. Zangeneh. Application of simulated annealing to inverse design of transonic turbomachinery cascades. Proceedings of The Institution of Mechanical Engineers Part A - Journal of Power and Energy, 216(A1):59-73, 2002.

[68] A. Veress and R.A. van den Braembussche. Inverse design and optimization of a return channel for a multistage centrifugal compressor. Journal of Fluids Engineering, 126(5):799-806, 2004. 
[69] F.C. Visser. On the flow in centrifugal impellers. PhD Thesis, University of Twente, 1996.

[70] L. de Vito, R.A. van den Braembussche, and H. Deconinck. A novel two-dimensional viscous inverse design method for turbomachinery blading. Journal of Turbomachinery, 125(5):310-316, 2003.

[71] Z.M. Wang, R.X. Cai, Chen H.J., and X.C. Jia. A three-dimensional inverse method using navier-stokes equations for turbomachinery blading. Inverse Problems in Engineering, 8(6):529-551, 2000.

[72] Water Features Direct Ltd. Pumps - Industrial. www.wfdasia.com.

[73] J. Westerweel. Fundamentals of digital particle image velocimetry. Measurement Science and Technology, 8(12):1379-1392, 1997.

[74] J. Westerweel. Theoretical analysis of the measurement precision in particle image velocimetry. Experiments in Fluids (Suppl.), 29:S3-S12, 2000.

[75] R.W. Westra, N.P Kruyt, K. van Andel, and H.W.M. Hoeijmakers. An optimization method for centrifugal pump impellers. International Symposium on Rotating Machinery 12, ISROMAC12-2008-20147:1-9, 2008.

[76] R.W. Westra, N.P Kruyt, and H.W.M. Hoeijmakers. An inverse-design method for centrifugal pump impellers. ASME 5th International Symposium on Turbomachinery, FEDSM2005-77283:1-8, 2005.

[77] G. Wuibaut, G. Bois, P. Dupont, G. Caignaert, and M. Stanislas. PIV measurements in the impeller and the vaneless diffuser of a radial flow pump in design and off-design operating conditions. Journal of Fluids Engineering, 124(3):791-797, 2002.

[78] G. Wuibaut, G. Bois, M. El Hajem, A. Akhras, and J.Y. Champagne. Optical PIV and LDV comparisons of internal flow investigations in SHF impeller. International Journal of Rotating Machinery, 2006:1-9, 2006.

[79] K.F.C. Yiu and M. Zangeneh. Three-dimensional automatic optimization method for turbomachinery blade design. Journal of Propulsion and Power, 16(6):1174-1181, 2000 .

[80] M. Zangeneh. A compressible three-dimensional design method for radial and mixed flow turbomachinery blades. International Journal for Numerical Methods in Fluids, 13(5):599-624, 1991.

[81] M. Zangeneh. Inverse design of centrifugal compressor vaned diffusers in inlet shear flows. Journal of Turbomachinery, 118(2):385-393, 1996.

[82] M. Zangeneh, A. Goto, and T. Takemura. Suppression of secondary flows in a mixedflow pump impeller by application of three-dimensional inverse design method: part 1. design and numerical validation. Journal of Turbomachinery, 118(3):536-543, 1996. 
[83] M. Zangeneh, M. Schleer, F. Ploger, S.S. Hong, C. Roduner, B. Ribi, and R.S. Abhari. Investigation of an inversely designed centrifugal compressor stage - part 1: Design and numerical verification. Journal of Turbomachinery, 126(1):73-81, 2004.

[84] O.C. Zienkiewicz and J.Z. Zhu. The superconvergent patch recovery and a posteriori error estimates. part 1: The recovery technique. International Journal for Numerical Methods in Engineering, 33(7):1331-1364, 1992. 



\section{APPENDIX A}

\section{Sensitivity analysis of optimization parameters}

In chapter 4 an optimization has been performed for a radial centrifugal pump impeller. In order to determine the influence of the optimization parameters and to investigate how optimal this design is, changes are made in these parameters to investigate the influence on the performance of the impeller. Firstly, the parameters describing the blade curvature are investigated. Secondly, the influence of the shroud curve parameters are discussed. Finally, the influence of the number of blades on the obtained optimum is studied.

The blade curvature is described by 10 (blade angle) parameters (see section 4.3.2). In order to investigate the influence of the blade curvature, all blade angle parameters of the optimum are changed by the same $\Delta \beta^{*}$ and the cost function is computed. The results are listed in table A.1. When the blade angle is increased, i.e. the blade becomes straighter,

Table A.1: Influence of blade curvature on the cost function

\begin{tabular}{|c|c|c|c|c|c|c|}
\hline$\Delta \beta^{*}$ & $F$ & $\Sigma c_{\psi} f_{\psi}$ & $\Sigma c_{\zeta} f_{\zeta}$ & $\Sigma c_{\kappa, w} f_{\kappa, w}$ & $\Sigma c_{\kappa, i} f_{\kappa, i}$ & back-flow? \\
\hline-2 & 1.68 & 0.02 & 0.74 & 0.60 & 0.35 & no \\
\hline-1 & 1.42 & 0.00 & 0.65 & 0.55 & 0.21 & no \\
\hline \hline 0 & 1.35 & 0.00 & 0.59 & 0.58 & 0.18 & no \\
\hline \hline 1 & 2.48 & 0.00 & 0.55 & 0.78 & 0.19 & $0.8 Q_{d}$ \\
\hline 2 & 3.26 & 0.00 & 0.52 & 1.05 & 0.30 & $0.8 Q_{d}$ \\
\hline
\end{tabular}

back-flow occurs at an earlier stage, and hence the cost function becomes very high. When the blade angle is reduced, i.e. the blade becomes more curved, most performance characteristics become less beneficial. Furthermore, by decreasing the blade angle, the pump head is lowered, and for $\Delta \beta^{*}=-2^{\circ}$ it can be seen that the head coefficient is smaller than the target. It is concluded that the found optimum is not improved by changing the blade curvature and that with respect to the blade curvature the design is indeed optimal for the current parameterization and formulation of the cost function.

The influence of changing the shroud curvature of the optimum is investigated by 
changing $\Delta z_{1}$. By changing this parameter, the width of the impeller is either decreased or increased when compared to the optimum. Results are given in table A.2. When the width of the impeller is increased, the relative velocity becomes smaller, resulting in lower boundary layer losses, but also a higher possibility for back-flow at the lower flow rate considered, leading to high values for the cost function. This effect can be clearly seen from table A.2. When the width of the impeller is decreased, the relative velocities increase, leading to higher boundary layer losses and lower pump heads. Therefore, it is concluded that the design is indeed optimal with respect to the shroud curvature for the selected parameterization and definition of the cost function.

Table A.2: Influence of meridional shroud curve on the cost function

\begin{tabular}{|c|c|c|c|c|c|c|}
\hline$\Delta z_{1} / D\left(10^{-3}\right)$ & $F$ & $\Sigma w_{\psi} f_{\psi}$ & $\Sigma w_{\zeta} f_{\zeta}$ & $\Sigma w_{\kappa, w} f_{\kappa, w}$ & $\Sigma w_{\kappa, i} f_{\kappa, i}$ & back-flow? \\
\hline-6.3 & 1.78 & 0.31 & 0.70 & 0.61 & 0.16 & no \\
\hline-1.3 & 1.40 & 0.00 & 0.64 & 0.59 & 0.17 & no \\
\hline \hline 3.7 & 1.35 & 0.00 & 0.59 & 0.58 & 0.18 & no \\
\hline \hline 8.7 & 2.17 & 0.00 & 0.55 & 0.61 & 0.18 & $0.8 Q_{d}$ \\
\hline 13.7 & 2.17 & 0.00 & 0.52 & 0.62 & 0.19 & $0.8 Q_{d}$ \\
\hline
\end{tabular}

An optimum value of 6 blades is found for the considered bounded range of $[4,6]$. In table A.3 the cost function is shown for a changing number of blades. When the number of blades is reduced, back flow occurs for those designs at the lower flow rate considered. Furthermore, the head does not exceed the head target, and hence these geometries are assigned a large cost function value. When the number of blades is increased the cost function can become lower than the optimum $o p t_{4,6}$. However, these values are outside the selected bounded range for the blade count, [4,6]. As noted in chapter 4, this bounded range has been selected for experimental reasons, i.e. for a good optical accessibility of the optimized impeller. Therefore, for the current parameterization (including bounded range) and formulation of the cost function the optimal number of blades was indeed found.

Table A.3: Influence of the number of blades on the cost function

\begin{tabular}{|c|c|c|c|c|c|c|}
\hline$Z$ & $F$ & $\Sigma w_{\psi} f_{\psi}$ & $\Sigma w_{\zeta} f_{\zeta}$ & $\Sigma w_{\kappa, w} f_{\kappa, w}$ & $\Sigma w_{\kappa, i} f_{\kappa, i}$ & back-flow? \\
\hline 4 & 3.68 & 0.97 & 0.67 & 1.04 & 0.41 & $0.8 Q_{d}$ \\
\hline 5 & 2.55 & 0.25 & 0.61 & 0.73 & 0.22 & $0.8 Q_{d}$ \\
\hline \hline 6 & 1.35 & 0.00 & 0.59 & 0.58 & 0.18 & no \\
\hline \hline 7 & 1.32 & 0.00 & 0.60 & 0.56 & 0.17 & no \\
\hline 8 & 1.43 & 0.00 & 0.62 & 0.59 & 0.23 & no \\
\hline
\end{tabular}

From the parameter sensitivity analysis it is noted that for the current selection of bounded ranges the optimization has converged adequately. Straightforward changes in the optimization parameters, within the bounded ranges, do not lead to a reduction in the cost function. 


\section{APPENDIX B}

\section{Vector plots of relative velocity}

In chapter 5 the PIV measurements are presented as contour plots of the magnitude of the relative velocity in the plane of measurement along with some streamlines. For a more detailed view of the measured flow fields at design condition $\left(Q=Q_{d}\right)$, the measured relative velocity vector plots are shown near hub and shroud in Fig. B.1 and B.2, respectively. In these figures a circular arc at $r / D=0.4$ spanning two impeller channels is also shown. This circular arc is used in chapter 5 in order to plot the relative velocity distribution from pressure to suction side.

The measured relative velocity vector fields are also shown at the lowest flow rate considered, $Q=0.5 Q_{d}$, since the largest deviations from potential flow theory occur at this flow rate. In Fig. B.3 the velocity vector field is shown in the measurement plane near the hub and in Fig. B.4 it is shown near the shroud. For brevity, the measured reatlive velocity vector fields at the other considered flow rates are not shown here. 


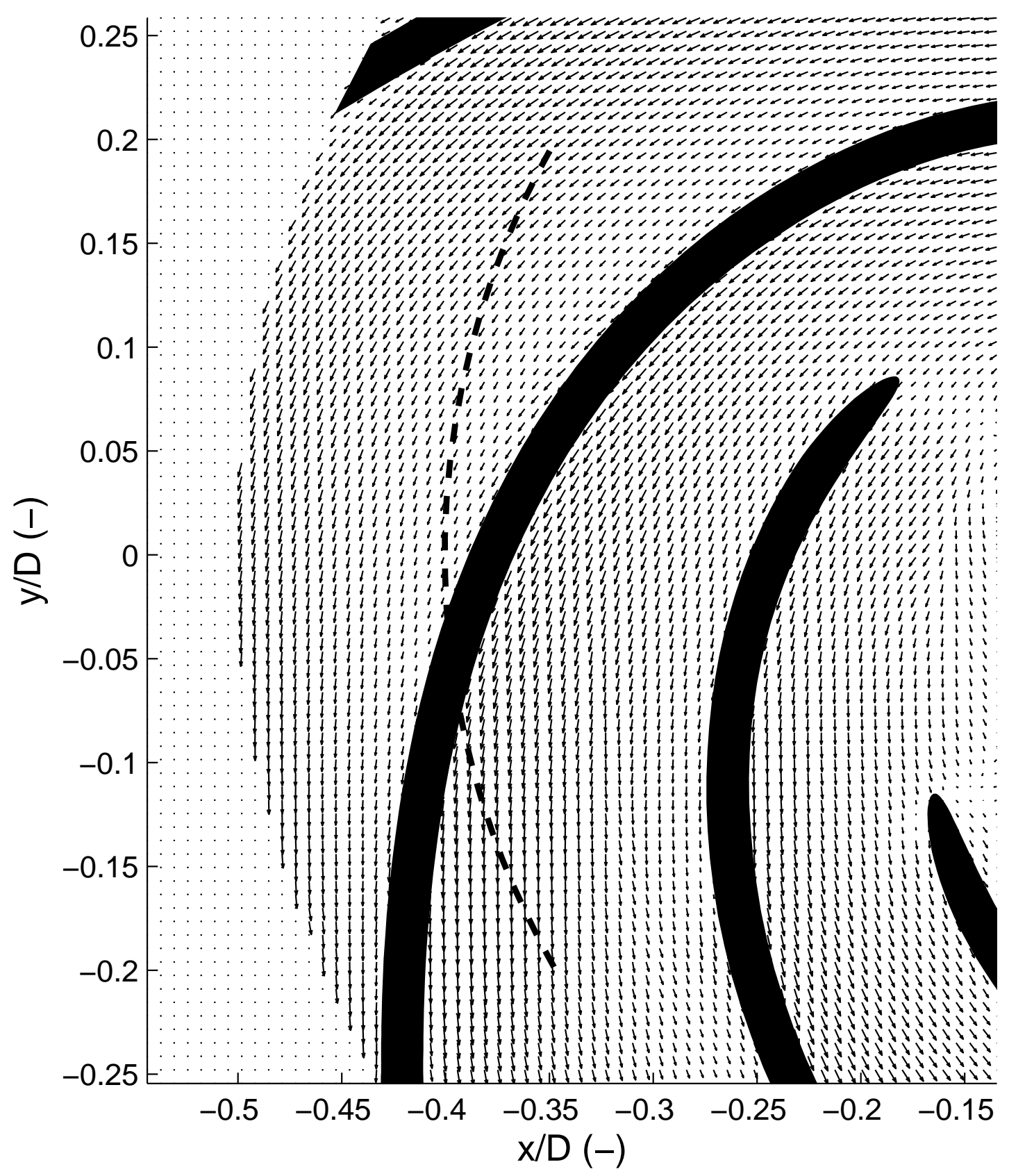

Figure B.1: Measured relative velocity vector field in a plane near the hub $(z / b=0.35)$ at $Q=Q_{d}$. The circular arc located at $r / D=0.4$ and indicated by the dashed line is used in chapter 5 to plot the relative velocity distribution from pressure to suction side. 


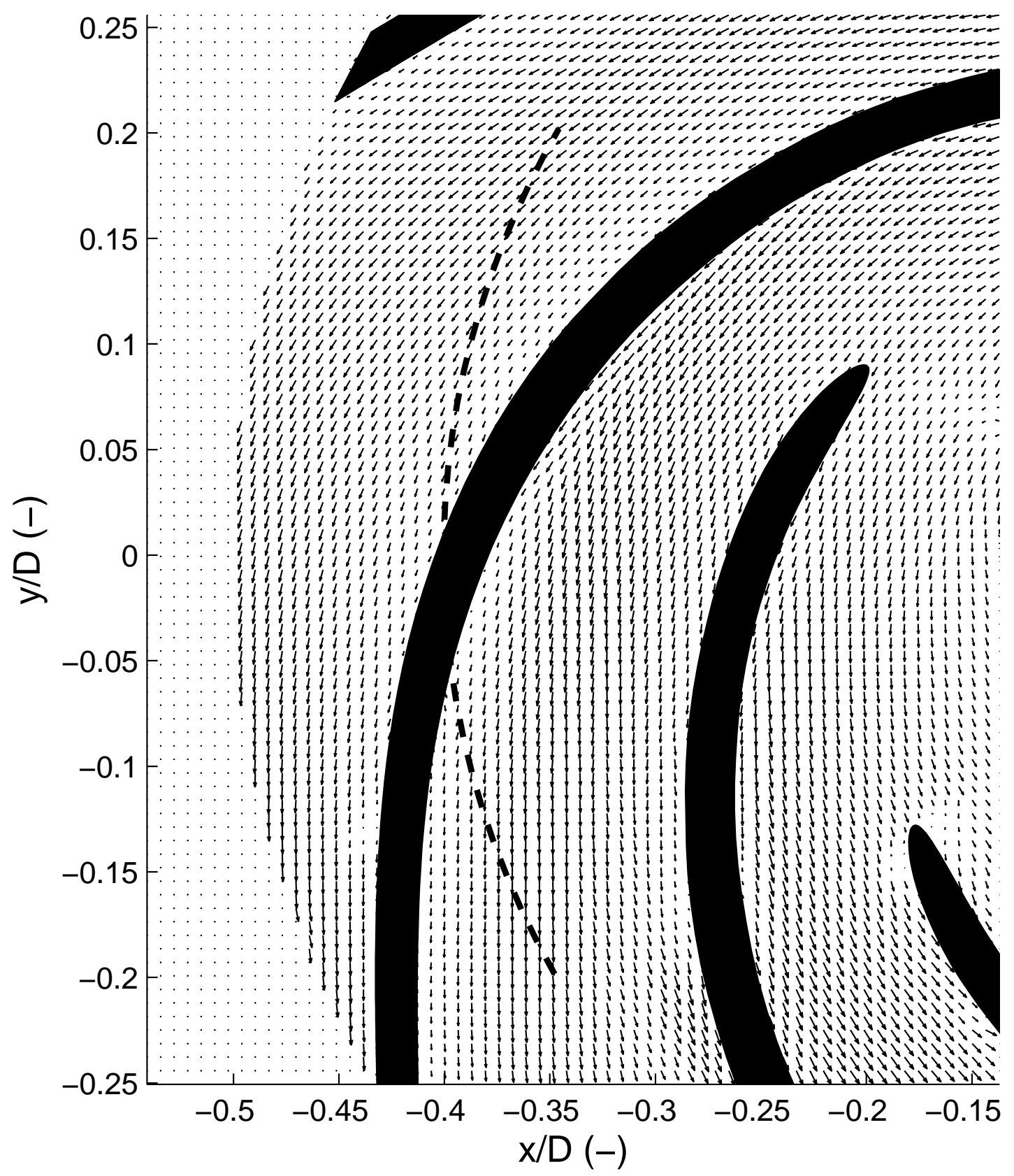

Figure B.2: Measured relative velocity vector field in a plane near the shroud $(z / b=0.85)$ at $Q=Q_{d}$. The circular arc located at $r / D=0.4$ and indicated by the dashed line is used in chapter 5 to plot the relative velocity distribution from pressure to suction side. 


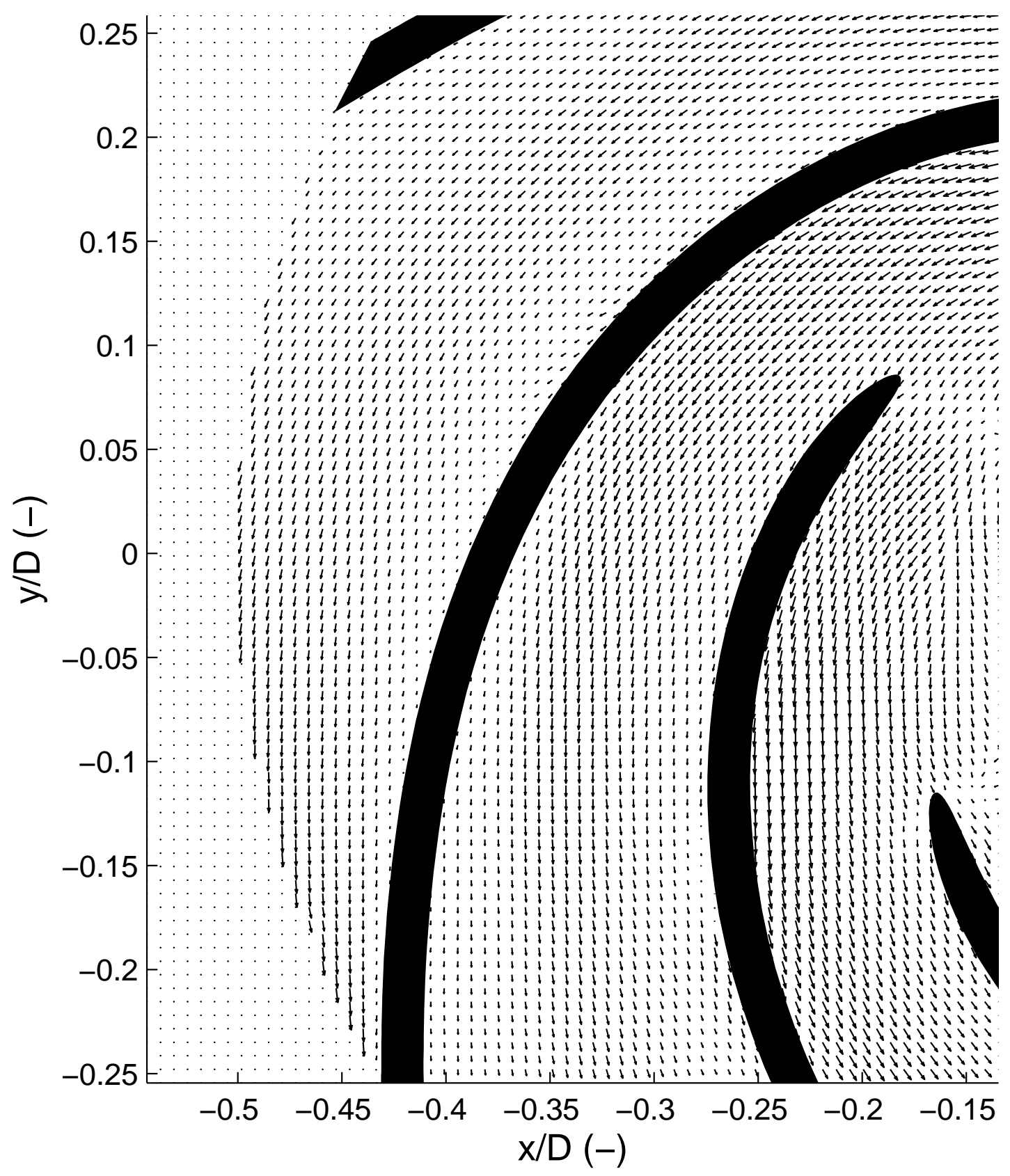

Figure B.3: Measured relative velocity vector field in a plane near the hub $(z / b=0.35)$ at $Q=0.5 Q_{d}$. 


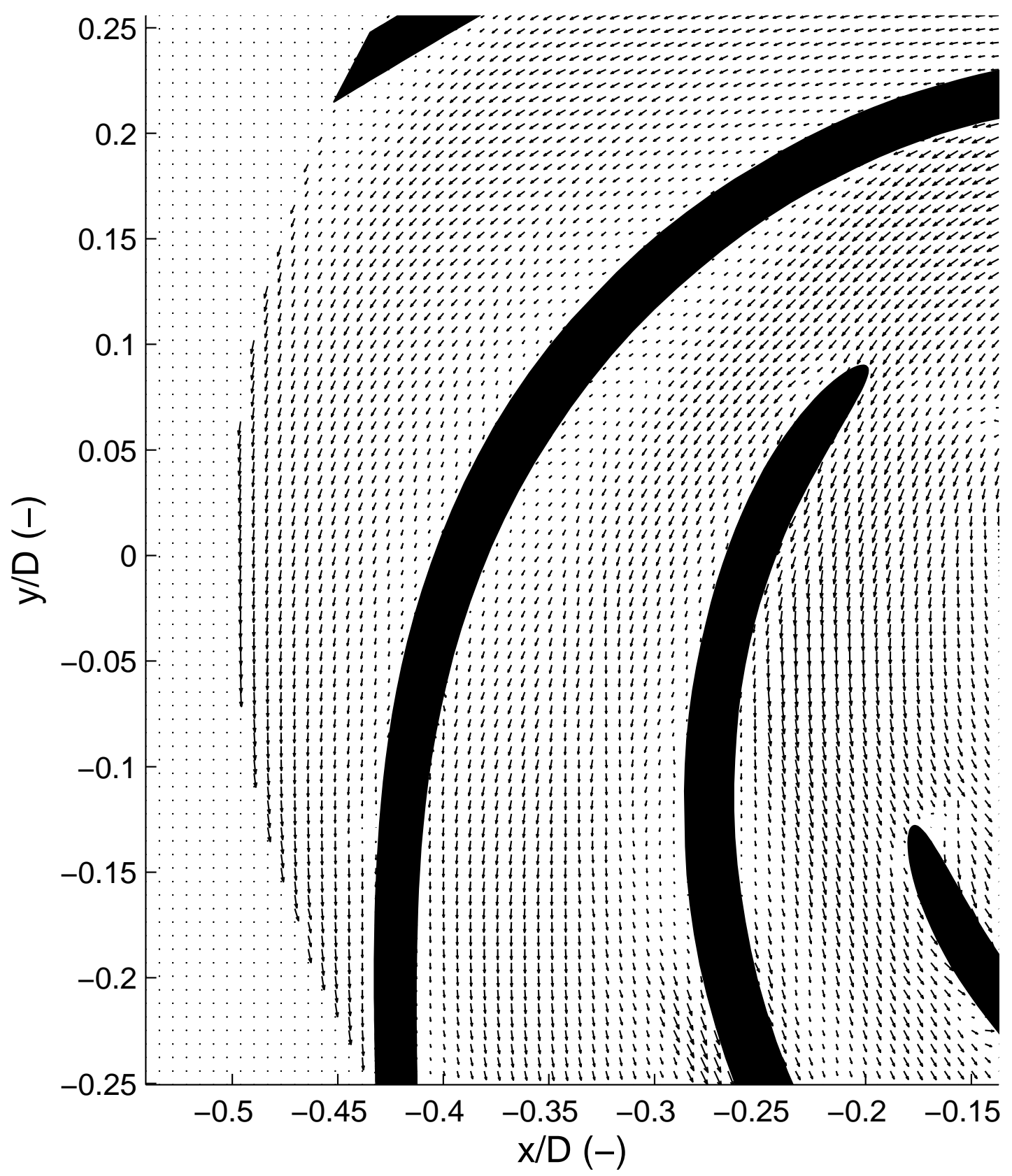

Figure B.4: Measured relative velocity vector field in a plane near the shroud $(z / b=0.85)$ at $Q=0.5 Q_{d}$. 



\section{APPENDIX C}

\section{Velocity measurements in centrifugal impellers}

In this appendix velocity measurements from literature carried out in centrifugal impellers are shown which are used for comparison with the results of the measurements executed in this work. This comparison is found in section 5.7.2.

In Fig. C.1 the LDV measurements by Van Os [47] in a mixed flow impeller are shown. Eckardt [28] performed measurements in a compressor impeller with straight blades and his results are depicted in Fig. C.2. Furthermore, Krain et al. [44] carried out measurements in a compressor impeller with backward curved blades and the results are displayed in Fig. C.3. The PIV measurements executed by Pedersen [48] are shown in Fig. C.4. Wuibaut et al. [78] executed LDV measurements in water and PIV measurements in air in the radial SHF impeller. The resulting velocity profiles at $r / r_{t e}=0.818$ are shown in Fig. C.5. Finally, the LDV measurements in a fifteen bladed impeller carried out by Visser [69] are presented in Fig. C.6. 


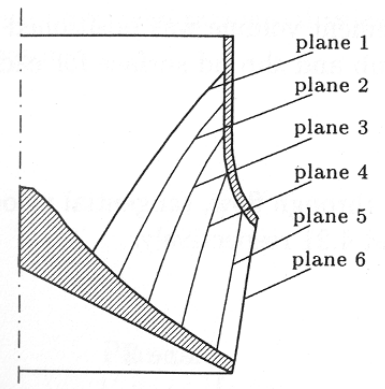

Plane 1

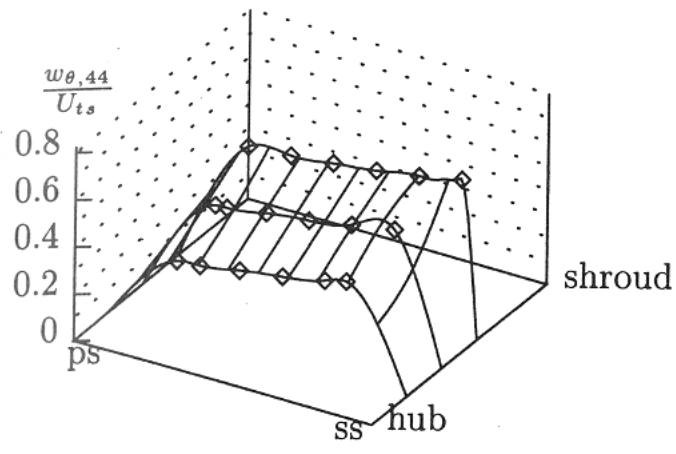

Plane 3

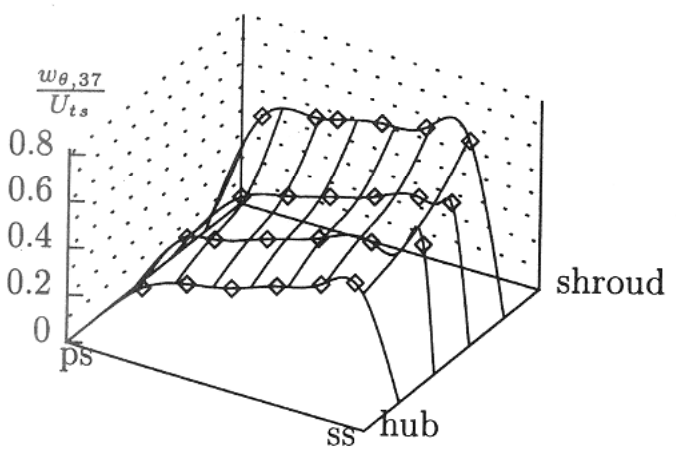

Plane 5

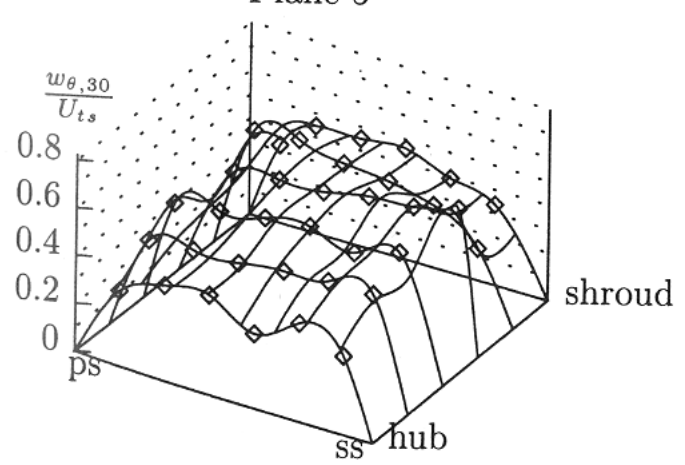

Plane 2

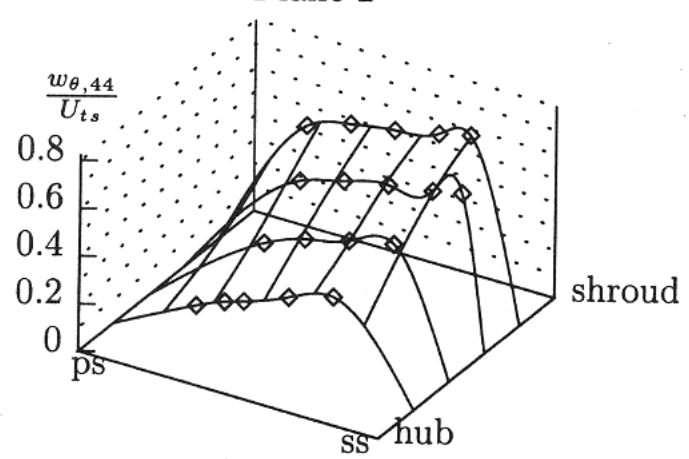

Plane 4

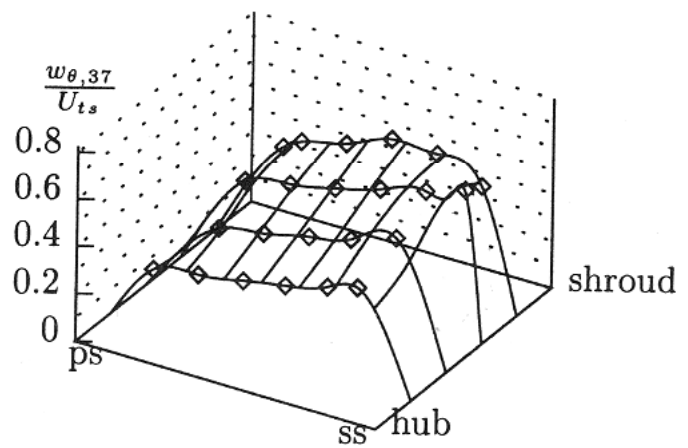

Plane 6

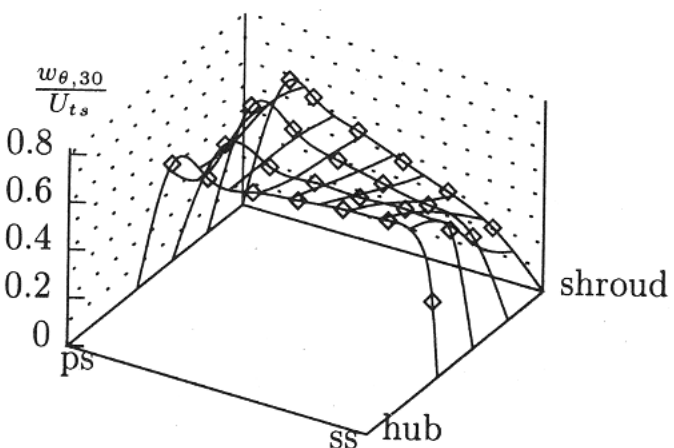

Figure C.1: Velocity distribution measured by Van Os [47] in a mixed flow pump impeller with LDV. 

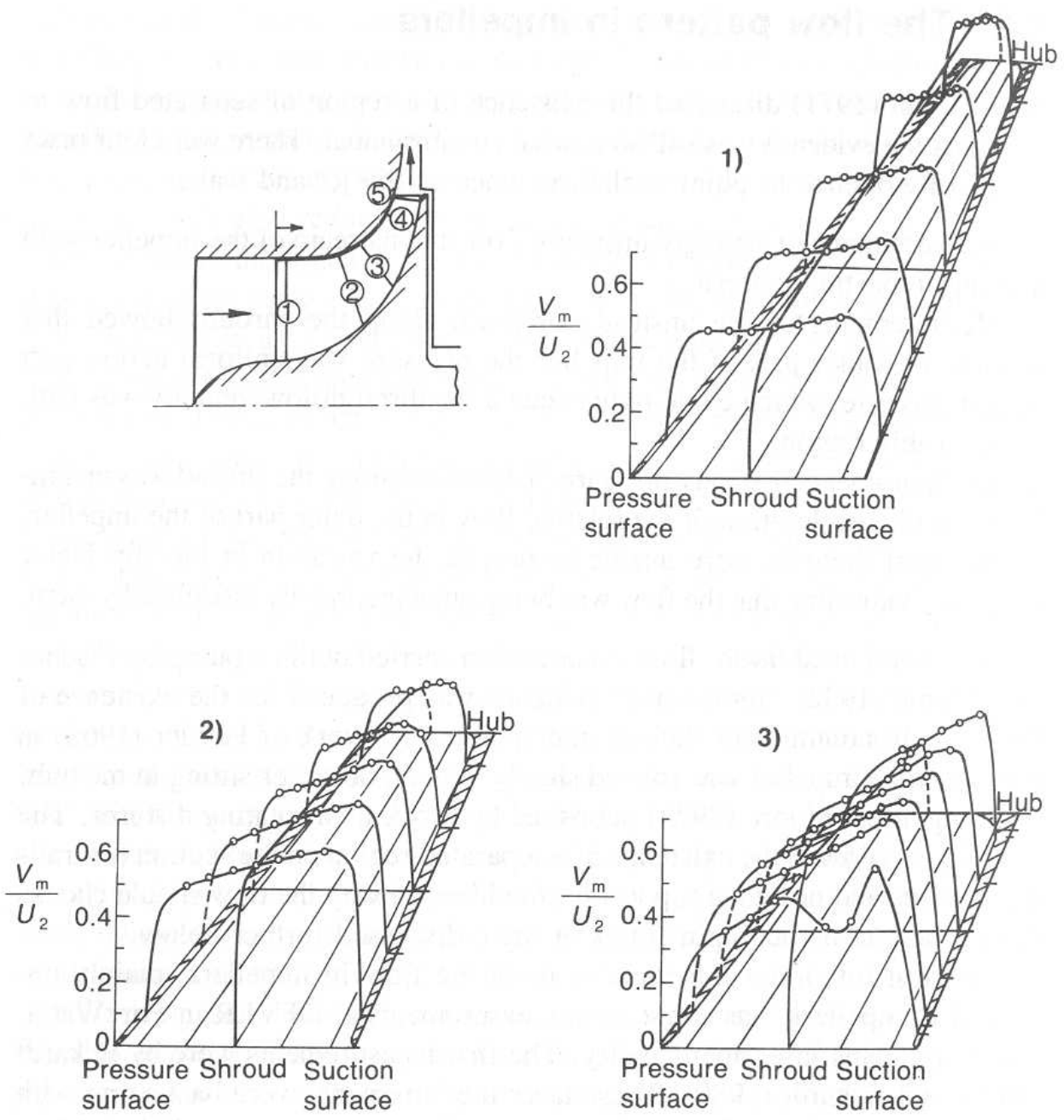

4)
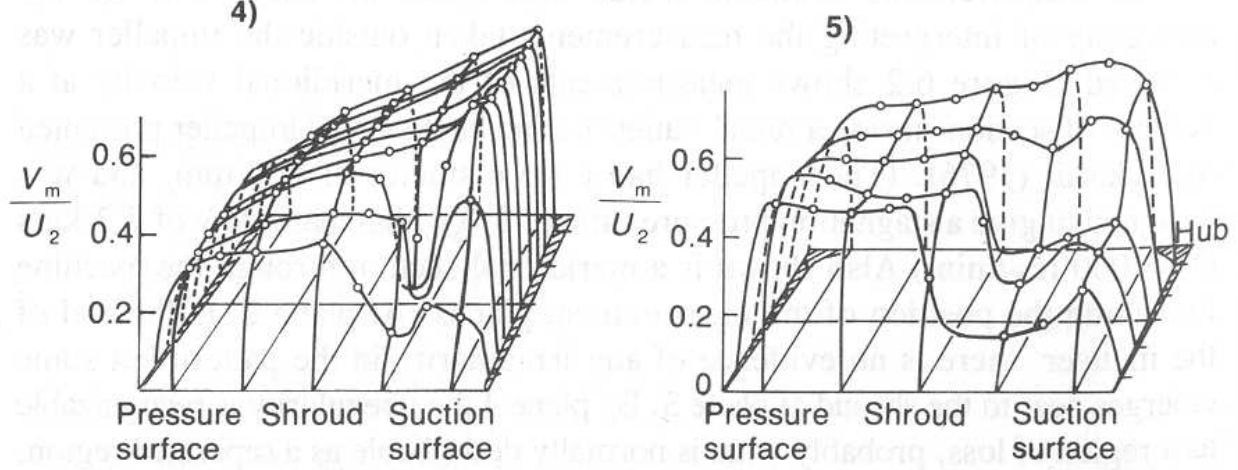

Figure C.2: Velocity distribution measured by Eckardt [28] in a compressor impeller with straight blades with LDV. Picture taken from [15]. 

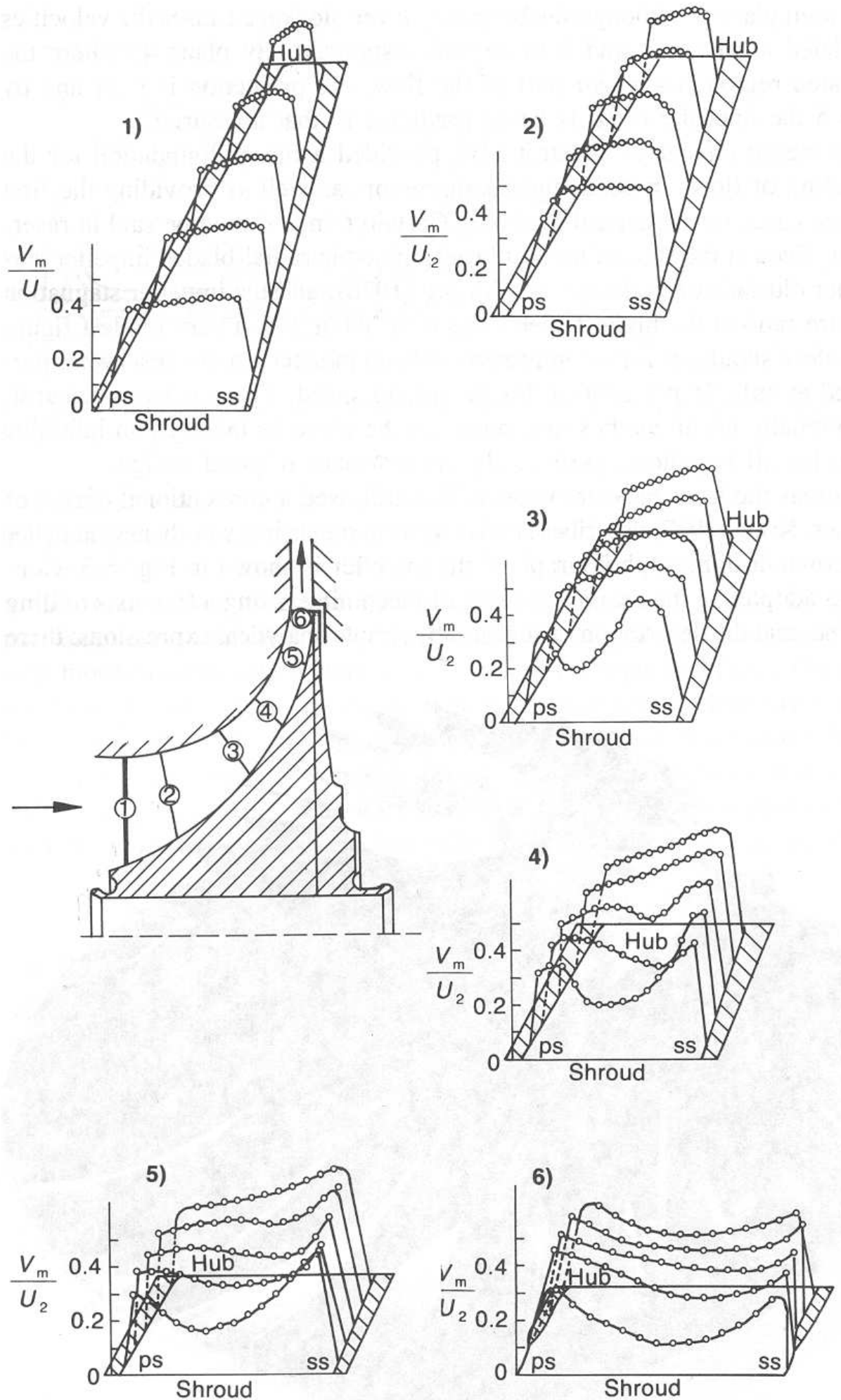

Figure C.3: Velocity distribution measured by Krain et al. [44] in a compressor impeller with backward curved blades with LDV. Picture taken from [15]. 


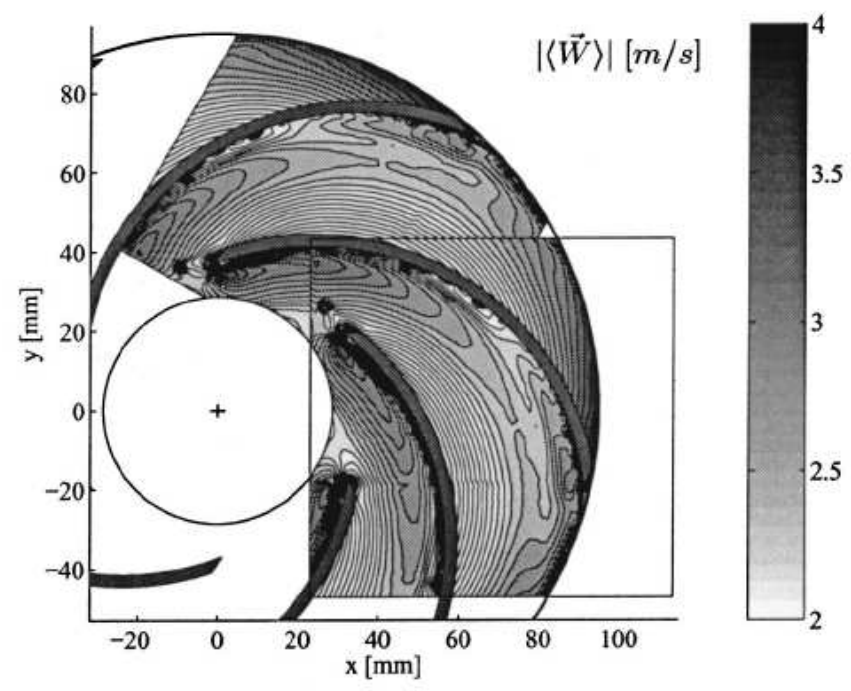

Figure C.4: Velocity distribution measurements by Pedersen [48] in a radial pump impeller with PIV.

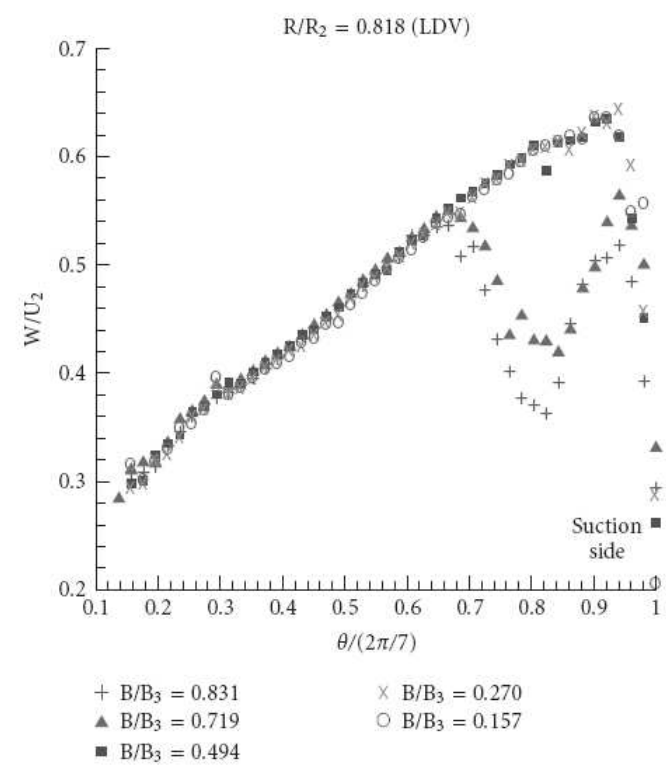

(a)

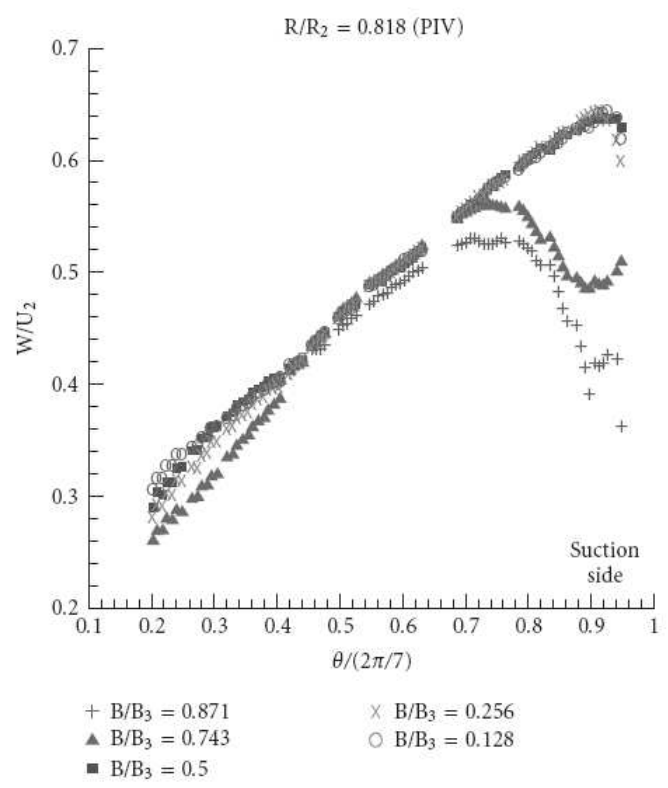

(b)

Figure C.5: Velocity distributions at $r / r_{t e}=0.818$ measured by Wuibaut et al. [78] with LDV and PIV. $B$ is the distance from hub to shroud: $B / B_{3}=0$ corresponds to the hub and $B / B_{3}=1$ corresponds to the shroud. 

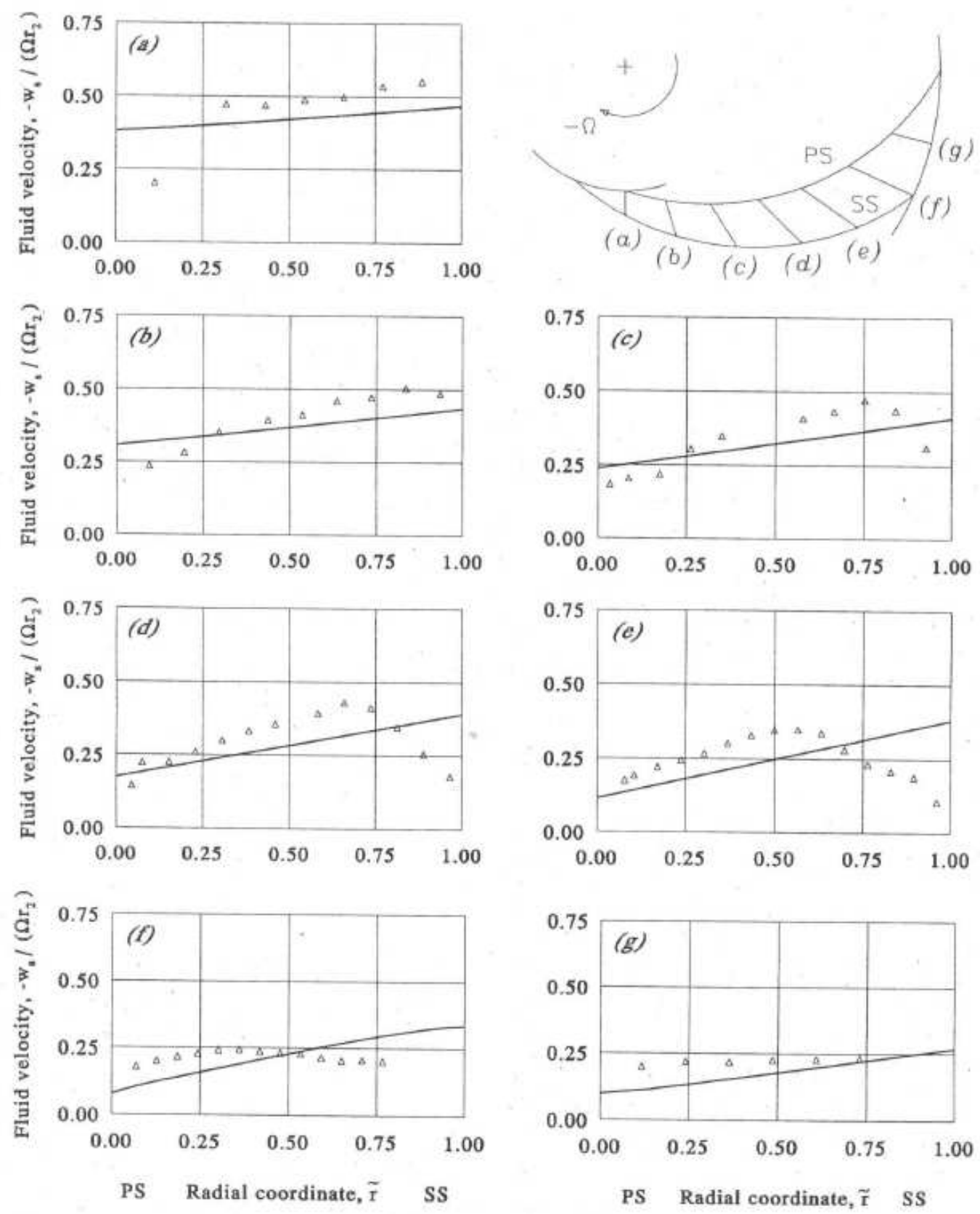

Figure C.6: Velocity distribution measured by Visser [69] in a fifteen bladed radial impeller using LDV. The straight lines indicate computed velocity profiles, whereas the triangles indicate the measurements. 


\section{Acknowledgments}

After more than four years I have finished my $\mathrm{PhD}$ thesis in the field of fluid mechanics in turbomachines. It has been a really enjoyable period in my life, and I would not have been able to finish this work without the help of many people, who I would like to thank here.

First of all, I would like to thank professor Harry Hoeijmakers for giving me the chance to do this $\mathrm{PhD}$ project. It has been a privilege for me to work with someone, with such an enormous know how in fluid mechanics. Our monthly meetings were very useful and always gave me good insight on how to tackle certain problems and how to proceed further. Also I would like to thank Harry for his contributions and feed-back on the progress reports, conference papers and this thesis.

Secondly, I would like to thank my mentor Niels Kruyt. It has been a huge pleasure to work with and learn from Niels, who like Harry has a tremendous knowledge in the field of fluid mechanics and more specifically also in the field of turbomachines. His door was always open for my questions and for brain storming sessions on how to address certain issues. The time he put in initializing the project and in reviewing progress reports, conference papers and this thesis is enormous, and it is very much appreciated.

Furthermore, during this project I have had the pleasure of working closely together with three MSc students. I would like to thank Nikolas Antonakis who worked on optimization methods for centrifugal pumps. My thanks also goes to Henny Bijleveld who worked on extending the inverse-design method with different loading functions and using a prescribed blade thickness. Last but not least, I would like to thank Koen van Andel, who contributed to building the experimental setup, and performing and evaluating the PIV measurements.

Designing and building the experimental setup has been the biggest challenge in this project. I would like to thank Guido Zwart and Chris Vos for designing the setup, and Chris Vos also for building the setup. Furthermore, I would like to thank Koen van Andel for building the experimental facility in cooperation with Chris Vos and succeeding in getting the PIV equipment operational. I still remember the drinks Chris, Koen and myself had on the day the construction of the setup was finished. It was a very unique collaboration and a lot of fun as well. My thanks also goes to Herman Stobbe who 
helped us greatly with problems that occurred during the building and operation of the setup. I would like to thank the following people also for their important contributions to the realization of the setup, Niels Kruyt, Harry Hoeijmakers and Jilles Eindhoven. Finally, I would like to thank everyone else at the Engineering Technology Department who contributed to this setup. We got a lot of help from a lot of people within the Department and that is greatly appreciated.

I want to thank STW for financing this project entitled Inverse-design and optimization methods for centrifugal pump impellers and fans (TSF.6157) and also the companies who were involved in the user committee for this STW project: Flowserve, IHC, Johnson Pump, Marin, NLR and Urenco Aerospace (Aeronamics). The progress meetings with the user committee were always very useful and appreciated.

I would like to thank IMPACT for providing the PIV equipment. In particular, I would like to thank Niels Deen for his help in using the PIV equipment, and his advice on performing PIV measurements. Also my thanks goes to Wim Leppink and Robert Meijer for their help with transporting the PIV equipment.

The Engineering Fluid Dynamics group is a really social group of permanent staff, PhD students and MSc students. The social activities, mainly borrels, were always fun, and especially the 'AIO vs. student lunch battles' were really enjoyable. Correct me if I am wrong, but I think in the end the result was a draw. I would like to thank my roommates Philip Kelleners, Krzysztof Wrobel and Jesse Slot for the nice working atmosphere. I would also like to thank my other PhD colleagues Peter van Dijk, Remco Habing, Jacco Hospers, Rutger IJzermans, Arjen Koop, George Popovici, Ryan Sidin, Arie Verhoeff, Huseyin Ozdemir, Hein de Vries and Marco van Zoelen. I would also like to express my gratitude to the permanent staff of the TS group. In particular I would like to thank our secretary Anjenet Mettivier-Meijer and our system administrator Wouter den Breeijen for helping me with various matters.

Last but not least, I would like to thank my family and friends for their support and interest during the last four and a half years. In particular I would like to thank my parents, Anne and Betty, and my brother and sisters, Anja, Linda, Christiaan and Marijke for their interest. 
Remko Willem Westra was born on the 14th of December 1976 in Zuidhorn. In 1987 he moved with his parents to Grou, where he finished primary school in 1989. He completed his secondary degree in 1995 at the Christelijk Gymnasium in Leeuwarden. In 1995 he started a study of Chemical Engineering at the University of Twente. Part of this study was an traineeship in 2000 at the Herakles General Cement Company in Volos, Greece. Here he studied the efficiencies of cyclones and the heat exchange in the rotary kiln, used for clinker production. In March 2003 he obtained his Master's degree at the Fundamentals of Chemical Reaction Engineering group of professor Hans Kuipers. The topic dealt with the study of the large-scale flow patterns occurring in gas-solid fluidized beds using the discrete bubble model.

In November 2003 he started his PhD assignment at the Engineering Fluid Dynamics group of professor Harry Hoeijmakers. The assignment was to develop inverse-design and optimization methods for turbomachines and perform measurements for validation. This is the topic of the thesis which lies before you now. Apart from attending several courses, he presented his work at the ASME 5th International Symposium on Pumping Machinery in Houston, June 2005, and at the International Symposium on Rotating Machinery, ISROMAC-12, in Honolulu, February 2008. 